\section{ECOHSTOR}

Make Your Publications Visible.
A Service of

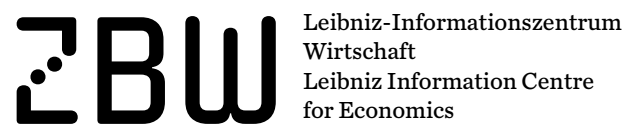

Cischinsky, Christiane

Book - Digitized Version

\title{
Auswirkungen der Europäischen Integration auf das deutsche Gesundheitswesen
}

Allokation im marktwirtschaftlichen System, No. 59

Provided in Cooperation with:

Peter Lang International Academic Publishers

Suggested Citation: Cischinsky, Christiane (2008) : Auswirkungen der Europäischen Integration auf das deutsche Gesundheitswesen, Allokation im marktwirtschaftlichen System, No. 59, ISBN 978-3-631-75556-3, Peter Lang International Academic Publishers, Berlin, http://dx.doi.org/10.3726/b14060

This Version is available at:

http://hdl.handle.net/10419/182642

Standard-Nutzungsbedingungen:

Die Dokumente auf EconStor dürfen zu eigenen wissenschaftlichen Zwecken und zum Privatgebrauch gespeichert und kopiert werden.

Sie dürfen die Dokumente nicht für öffentliche oder kommerzielle Zwecke vervielfältigen, öffentlich ausstellen, öffentlich zugänglich machen, vertreiben oder anderweitig nutzen.

Sofern die Verfasser die Dokumente unter Open-Content-Lizenzen (insbesondere CC-Lizenzen) zur Verfügung gestellt haben sollten, gelten abweichend von diesen Nutzungsbedingungen die in der dort genannten Lizenz gewährten Nutzungsrechte.
Terms of use:

Documents in EconStor may be saved and copied for your personal and scholarly purposes.

You are not to copy documents for public or commercial purposes, to exhibit the documents publicly, to make them publicly available on the internet, or to distribute or otherwise use the documents in public.

If the documents have been made available under an Open Content Licence (especially Creative Commons Licences), you may exercise further usage rights as specified in the indicated licence. 
CHRISTIANE CISCHINSKY

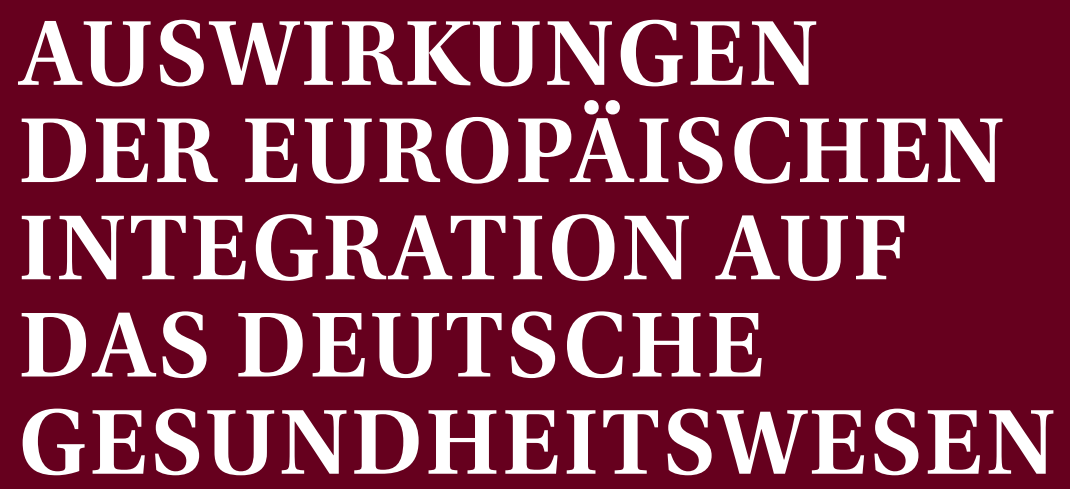




\section{CHRISTIANE CISCHINSKY}

\section{AUSWIRKUNGEN DER EUROPÄISCHEN INTEGRATION AUF DAS DEUTSCHE GESUNDHEITSWESEN}

In den vergangenen Jahrzehnten haben sich durch die Europäische Integration zahlreiche Einflüsse auf das deutsche Gesundheitswesen ergeben. Hierzu zählt neben den Aktionsprogrammen der Europäischen Union zur Verbesserung der öffentlichen Gesundheit und der Anwendung der Offenen Methode der Koordinierung auf den Bereich der Gesundheitspolitik insbesondere auch die Rechtsprechung des Europäischen Gerichtshofes zur grenzüberschreitenden Inanspruchnahme von Gesundheitsleistungen. Die Arbeit stellt diese Entwicklungen dar und untersucht bisherige und in der Zukunft zu erwartende Auswirkungen auf die deutsche Gesundheitspolitik und das Gesundheitswesen.

Christiane Cischinsky, 1972 in Saarbrücken geboren, studierte von 1992 bis 1997 Volkswirtschaftslehre an der Universität des Saarlandes. Von 1999 bis 2007 arbeitete sie als Wissenschaftliche Mitarbeiterin am Lehrstuhl für Volkswirtschaftslehre, insbesondere Planung und Verwaltung öffentlicher Wirtschaft der Universität Mannheim. Seit Ende 2007 ist sie am Dekanat der volkswirtschaftlichen Abteilung der Universität Mannheim als Wissenschaftliche Mitarbeiterin tätig. 
Auswirkungen der Europäischen Integration auf das deutsche Gesundheitswesen 


\section{ALLOKATION IM MARKTWIRTSCHAFTLICHEN SYSTEM \\ Herausgegeben von \\ Heinz König ( $\dagger$ ), Hans-Heinrich Nachtkamp, \\ Ulrich Schlieper, Eberhard Wille}

Band 59

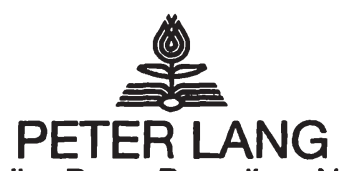

Frankfurt am Main · Berlin · Bern · Bruxelles - New York · Oxford · Wien 


\section{CHRISTIANE CISCHINSKY}

\section{AUSWIRKUNGEN \\ DER EUROPÄISCHEN \\ INTEGRATION \\ AUF DAS DEUTSCHE \\ GESUNDHEITSWESEN}

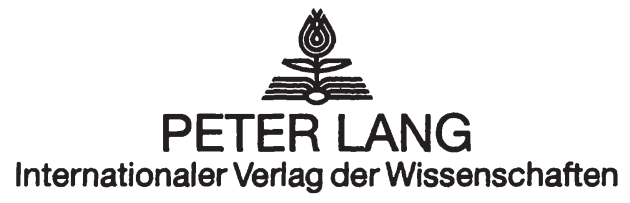


Bibliografische Information der Deutschen Nationalbibliothek Die Deutsche Nationalbibliothek verzeichnet diese Publikation in der Deutschen Nationalbibliografie; detaillierte bibliografische Daten sind im Internet über <http://www.d-nb.de> abrufbar.

Open Access: The online version of this publication is published on www.peterlang.com and www.econstor.eu under the international Creative Commons License CC-BY 4.0. Learn more on how you can use and share this work: http://creativecommons.org/licenses/ by/4.0.

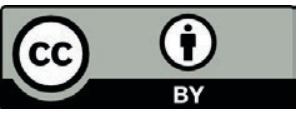

This book is available Open Access thanks to the kind support of ZBW - Leibniz-Informationszentrum Wirtschaft.

Zugl.: Mannheim, Univ., Diss., 2007

Gedruckt auf alterungsbeständigem, săurefreiem Papier.

\author{
D 180 \\ ISSN 0939-7728 \\ ISBN 978-3-631-57829-2 \\ ISBN 978-3-631-75556-3 (eBook) \\ (c) Peter Lang GmbH \\ Internationaler Verlag der Wissenschaften \\ Frankfurt am Main 2008
}




\section{Vorwort}

Die vorliegende Dissertation entstand während meiner Tätigkeit am Lehrstuhl für Volkswirtschaftslehre, insbesondere Planung und Verwaltung offentlicher Wirtschaft, der Universität Mannheim. Sie wurde im Herbstsemester 2007 von der Fakultät für Rechtswissenschaft und Volkswirtschaftslehre, Abteilung Volkswirtschaftslehre, als Dissertation angenommen.

Ich möchte mich an dieser Stelle sehr herzlich bei meinem Doktorvater Prof. Dr. Eberhard Wille bedanken. Seine Anregungen und seine Hilfe haben wesentlich zum Gelingen dieser Dissertation beigetragen. Prof. Dr. Jürgen Schröder war so freundlich, das Zweitgutachten übernehmen, wofür ich mich bei ihm herzlich bedanken möchte.

Bedanken möchte ich mich auch bei meinen ehemaligen wissenschaftlichen Kolleginnen und Kollegen, den studentischen Hilfskräften und den Sekretärinnen am Lehrstuhl von Herm Prof. Dr. Wille. Sie alle haben wesentlich zur guten Arbeitsatmosphäre am Lehrstuhl beigetragen.

Ein herzlicher Dank gilt meinen Eltern und Schwiegereltern sowie meinen Geschwistern, die meine Ausbildung mit liebevollem Interesse begleitet und mich in vielfaltiger Weise unterstützt haben. Meinem Schwiegervater und meiner Schwester dankte ich darüber hinaus für die Hilfe bei der Korrektur des Manuskriptes. Ganz besonders möchte mich aber bei meinem Mann bedanken, der mir durch sein Verständnis und seine Geduld während der gesamten Dissertationsphase eine unverzichtbare Hilfe war und somit wesentlich zum Gelingen der Arbeit beigetragen hat.

Mannheim, im April 2008

Christiane Cischinsky 



\section{Inhaltsverzeichnis}

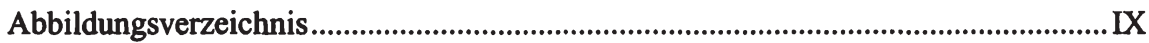

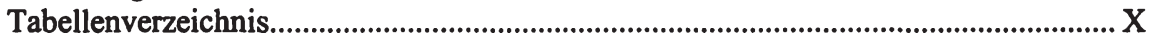

Abkürzungsverzeichnis ..........................................................................................................XII

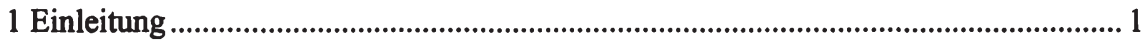

2 Wirkungen der Europäischen Integration im Gesundheitsbereich ............................. 5

2.1 Entwicklung der Kompetenzen der Europäischen Union ................................... 5

2.2 Aktionsprogramme im Bereich der öffentlichen Gesundheit ............................9

2.3 Bedeutung der europäischen Grundfreiheiten im Gesundheitswesen............. 12

2.3.1 Freiheit des Personenverkehrs............................................................ 13

2.3.1.1 Niederlassungsfreiheit .............................................................. 13

2.3.1.2 Arbeitnehmerfreizügigkeit......................................................... 16

2.3.2 Freiheit des Warenverkehrs ............................................................. 18

2.3.3 Freiheit des Dienstleistungsverkehrs................................................... 23

2.3.3.1 EuGH-Rechtsprechung zur passiven Dienstleistungsfreiheit

2.3.3.2 Die aktive Dienstleistungsfreiheit für Angehörige von Gesundheitsberufen........................................................... 31

2.4 Zur Geltung des europäischen Wettbewerbsrechts ............................................ 33

2.5 Offene Methode der Koordinierung...................................................................... 38

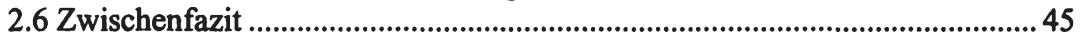

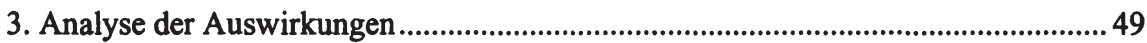

3.1 Vorbemerkungen 58

3.2 Ambulante und stationäre Versorgung..............................................................49

3.2.1 Patientenmobilität.................................................................................... 49

3.2.2 Ambulante Versorgung ........................................................................ 57

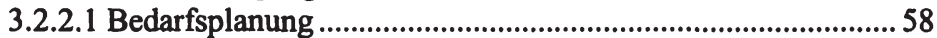

3.2.2.2 Vertragsärztliche Vergütung...................................................67 67

3.2.2.2.1 Varianten der vertragsärztlichen Vergütung.................67 67

3.2.2.2.2 Vertragsärztliche Vergütung in Deutschland............... 72

3.2.2.2.3 Europatauglichkeit der betrachteten Systeme und Änderungsvorschläge...................................................... 76

3.2.2.3 Veranlasste Leistungen ..................................................... 79

3.2.3 Stationäre Versorgung .............................................................................. 83

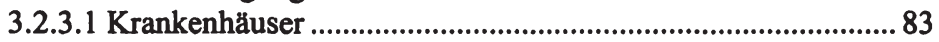

3.2.3.2 Vorsorge- und Rehabilitationseinrichtungen......................... 90

3.3 Arzneimittel- und Apothekenmarkt ................................................................. 101

3.3.1 Arzneimittelmarkt........................................................................... 101

3.3.1.1 Europäische Marktzulassungsregelungen.............................. 103

3.3.1.2 Schutzrechte ............................................................................... 104 
3.3.1.3 Einflussnahmeversuche der EU auf nationale Kostendämpfungsmaßnahmen

3.3.1.4 Nationale Kostendämpfungsmaßnahmen im Arzneimittelmarkt

3.3.1.4.1 Direkte Preisregulierung ........................................... 111

3.3.1.4.2 Indirekte Preisregulierungen .................................... 112

3.3.1.4.3 Maßnahmen zur Mengensteuerung........................... 114

3.3.1.4.4 Exkurs: Arzneimittelpreise im internationalen

Vergleich ............................................................116

3.3.1.4.5 Parallel- und Reimporte ............................................... 120

3.3.1.4.6 Generikaförderung .................................................. 126

3.3.1.5 Auswirkungen einer steigenden Patientenmobilität auf die Arzneimittelhersteller............................................ 133

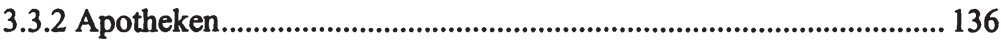

3.3.2.1 Situation auf dem Apothekenmarkt...................................... 141

3.3.2.2 Zuzahlungen und Versandapotheken..................................... 152

4 Gesundheitssysteme im Wettbewerb ...................................................................... 161

5 Fazit

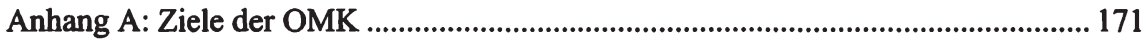

Anhang B: Daten zur Patientenmobilität ...............................................................175

Anhang C: Daten zur Finanzierung der Gesundheitsausgaben...................................... 182

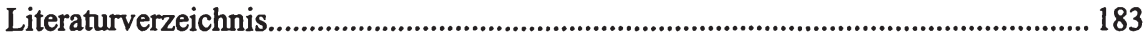




\section{Abbildungsverzeichnis}

Abbildung 1: Anzahl offener Planungsbereiche in den Kassenärztlichen

Vereinigungen Anfang 2006

Abbildung 2: Niederlassungsmöglichkeiten nach Arztgruppen (Anfang 2006)

Abbildung 3: Entwicklung der Altersstruktur der Vertragsärzte

Abbildung 4: Umsatzanteile der Generika an den nationalen

Arzneimittelmårkte 2001 in Prozent

Abbildung 5: Vergleich der Apothekenspannen vor und nach dem GMG (in $€$ )

Abbildung 6: Entwicklung der Apothekenzahl

Abbildung 7: Einwohner pro öffentliche Apotheke 1996, internationaler

Vergleich

Abbildung 8: Laspeyres-Preisindex des Apothekenabgabepreises

(Vergleichsmaßstab: Devisenkurs)

Abbildung 9: Selbstbeteiligung der Patienten auf Arzneimittel in Deutschland.

Abbildung 10: Vergleich der Selbstbeteiligung der Patienten für rezeptpflichtige Arzneimittel 156

Abbildung 11: Gesundheitsquoten im europäischen Vergleich 2002 (in Prozent des BIP) 164

Abbildung 12: Zusammenhang zwischen dem Anteil der öffentlichen Gesundheitsausgaben am BIP und der Bewertung der Gesundheitssysteme 165

Abbildung 13: Korrelation zwischen dem Finanzierungsanteil der Sozialversicherung an den gesamten Gesundheitsausgaben und der Zufriedenheit der Bürger mit ihrem Gesundheitssystem ... 166 



\section{Tabellenverzeichnis}

Tabelle 1: Eckzahlen der stationären Versorgung in Deutschland von $1991-2003$.

Tabelle 2: $\quad$ Einrichtungen, Betten und Patientenbewegung in Vorsorge- und Rehabilitationseinrichtungen, $1991-2005$

Tabelle 3: Übersicht über Vorsorge- und Rehabilitationsleistungen. 93

Tabelle 4: Instrumente der Kostendämpfung im Arzneimittelsektor.

Tabelle 5: Internationaler Vergleich der Herstellerabgabepreise

Tabelle 6: Internationaler Vergleich der Apothekenabgabepreise.

Tabelle 7: Umsatzsteuersätze in ausgewählten europäischen Ländern

Tabelle 8: $\quad$ Preisdifferenz bei erstattungsfähigen Arzneimitteln durch Handelsaufschläge und Umsatzsteuer (USt) am Beispiel eines Medikaments mit einem europa-einheitlichen Herstellerabgabepreis von $10 €$.

Tabelle 9: Umsatzanteil der Parallelimporte am verschreibungspflichtigen Markt in ausgewählten europäischen Ländern, 1997 - 2002

Tabelle 10: Maßnahmen zur Förderung der Generikanachfrage . 128

Tabelle 11: Übernahmen im Generikamarkt.

Tabelle 12: Anteile des Herstellerabgabepreises, der Vertriebskosten und der Umsatzsteuer am Apothekenverkaufspreis (AVP) im EU-Vergleich

Tabelle 13: Abschätzung der Einsparmöglichkeiten durch den Einkauf von Arzneimitteln im Ausland für das Jahr 2005

Tabelle I: Vorschlag für gemeinsame Ziele im Bereich Gesundheitswesen und Langzeitpflege (OMK).

Tabelle II: Ziele für die gestraffte OMK in den Bereichen „Soziale Eingliederung", „Renten“ und „Gesundheitsversorgung und Langzeitpflege“... 
Tabelle III: Zahl der Staatsangehörigen eines anderen Mitgliedstaates, die als Patienten im jeweiligen Mitgliedstaat behandelt wurden

Tabelle IV: Zahl der Personen, die eine Genehmigung einer Behandlung im Ausland beantragt haben........................................................................... 177

Tabelle V: Prozentsatz der erteilten Genehmigungen und Zahl der beantragten Erstattungen ohne Genehmigung............................................................ 180

Tabelle VI: Anteil der verschiedenen Finanzierungsträger an den Gesundheitsausgaben, 2003 
Abkürzungsverzeichnis

AABG Gesetz zur Begrenzung der Arzneimittelausgaben der gesetzlichen Krankenversicherung (Arzneimittelausgaben-Begrenzungsgesetz)

ABAG Gesetz zur Ablösung des Arznei- und Heilmittelbudgets (Arzneimittelbudget-Ablösegesetz)

ABDA Bundesvereinigung Deutschen Apothekerverbände

$\mathrm{ABl}$.

ABPI

Abs.

AEP

AG

Amtsblatt

AMG

Association of the British Pharmaceutical Industry

Absatz

Apothekeneinkaufspreis

Aktiengesellschaft

AMPreisV

Gesetz liber den Verkehr mit Arzneimitteln (Arzneimittelgesetz)

AOK

Arzneimittelpreisverordnung

ApBetro

Allgemeine Ortskrankenkasse

ApoG

ArbZG

Art.

Verordnung über den Betrieb von Apotheken (Apothekenbetriebsordnung)

AVP

Gesetz über das Apothekenwesen (Apothekengesetz)

Arbeitszeitgesetz

Artikel

Basys

BEUG

BfA

Apothekenverkaufspreis

Beratungsgesellschaft für angewandte Systemforschung

Bureau Européen des Unions die Consommateurs

BFB

Bundesagentur für Arbeit

BMG

Bundesverband der Freien Berufe

BMGS

Bundesministerium für Gesundheit

BPI

Bundesministerium furr Gesundheit und Soziale Sicherung

BSSichG

Bundesverband der Pharmazeutischen Industrie

Gesetz zur Sicherung der Beitragssätze in der gesetzlichen Krankenversicherung und in der gesetzlichen Rentenversicherung (Beitragssatzsicherungsgesetz)

BvDP Bundesverband Deutscher Postdienstleister

c.p. ceteris paribus

d.h. das heisst

DHV Deutscher Heilbäderverband

DKG Deutsche Krankenhausgesellschaft

DRGs Diagnosis Related Groups (diagnosebezogenen Fallpauschalen)

DVKA Deutschen Verbindungsstelle Krankenversicherung Ausland

EAG Europäische Atomgemeinschaft

EBM Einheitlicher Bewertungsmaßstab

EBS Europäische Beschäftigungsstrategie

endg. endgültig

EG

EGKS

Europäische Gemeinschaften, Europäische Gemeinschaft

EGV

Europäische Gemeinschaft für Kohle und Stahl

Vertrag zur Gründung der Europäischen Gemeinschaft 


\begin{tabular}{|c|c|}
\hline Einw. & Einwohner \\
\hline EMEA & $\begin{array}{l}\text { European Medicines Agency (Europäische Arzneimittelagentur), } \\
\text { bis } 2004 \text { European Agency for the Evaluation of Medicinal Products } \\
\text { (Europäische Agentur für die Beurteilung von Medikamenten) }\end{array}$ \\
\hline EPA & Europäisches Patentamt \\
\hline EPO & Europäische Patentorganisation \\
\hline EPÜ & Europäisches Patentübereinkommen \\
\hline ESF & Europäischer Sozialfonds \\
\hline EU & Europäische Union \\
\hline EuG & Gericht erster Instanz des Europäischen Gerichtshofes \\
\hline EuGH & Gerichtshof der Europäischen Gemeinschaft \\
\hline Euratom & Europäische Atomgemeinschaft \\
\hline EWG & Europäische Wirtschaftsgemeinschaft \\
\hline EWGV & Vertrag zur Gründung der Europäischen Wirtschaftsgemeinschaft \\
\hline EWR & Europäischer Wirtschaftsraum \\
\hline f & folgende \\
\hline ff & fortfolgende \\
\hline Fn. & Fußnote \\
\hline GASP & Gemeinsamen Außen- und Sicherheitspolitik \\
\hline GG & Grundgesetz \\
\hline ggf. & gegebenenfalls \\
\hline GKV & Gesetzliche Krankenversicherung \\
\hline GMG & $\begin{array}{l}\text { Gesetz zur Modernisierung der Gesetzlichen Krankenversicherung } \\
\text { (GKV-Modernisierungsgesetz) }\end{array}$ \\
\hline GRG & $\begin{array}{l}\text { Gesetz zur Strukturreform im Gesundheitswesen (Gesundheits-Re- } \\
\text { formgesetz) }\end{array}$ \\
\hline GSG & $\begin{array}{l}\text { Gesetz zur Sicherung und Strukturverbesserung der gesetzlichen } \\
\text { Krankenversicherung (Gesundheits-Strukturgesetz) }\end{array}$ \\
\hline GVG & Gesellschaft für Versicherungswissenschaft und -gestaltung \\
\hline HEK & Hanseatische Krankenkasse \\
\hline HVM & Honorarverteilungsmaßstab \\
\hline HWG & $\begin{array}{l}\text { Gesetz über die Werbung auf dem Gebiete des Heilwesens (Heil- } \\
\text { mittelwerbegesetz) }\end{array}$ \\
\hline i.d.R. & in der Regel \\
\hline i.V.m. & in Verbindung mit \\
\hline IKK & Innungskrankenkasse \\
\hline KBV & Kassenärztliche Bundesvereinigung \\
\hline KFPV 2004 & $\begin{array}{l}\text { Verordnung zum Fallpauschalensystem für Krankenhäuser für das } \\
\text { Jahr } 2004\end{array}$ \\
\hline KHG & $\begin{array}{l}\text { Gesetz zur wirtschaftlichen Sicherung der Krankenhäuser und zur } \\
\text { Regelung der Krankenhauspflegesätze (Krankenhausfinanzierungs- } \\
\text { gesetz) }\end{array}$ \\
\hline KV & Kassenärztliche Vereinigung \\
\hline Ven & Kassenärztliche Vereinigungen \\
\hline lit. & litera (lat. Buchstabe) \\
\hline
\end{tabular}




\begin{tabular}{ll} 
LMI & Legemiddelindustrif (Norwegischer Verband der Arzneimittelher- \\
& steller) \\
Mio. & Millionen \\
Mrd. & Milliarden \\
NHS & National Health Service \\
o.V. & ohne Verfasser \\
ÖBIG & Österreichisches Bundesinstitut für Gesundheitswesen \\
OECD & Organisation for Economic Cooperation and Development \\
OMK & offene Methode der Koordinierung \\
OTC & Over the Counter \\
PatG & Patentgesetz \\
PCT & Primary Care Trust \\
PJZS & polizeilichen und justiziellen Zusammenarbeit im Strafrecht \\
PPRS & Pharmaceutical Price Regulation Scheme \\
RLV & Regelleistungsvolumina \\
Rn. & Randnummer \\
S. & Seite \\
SGB & Sozialgesetzbuch \\
SPC & Ausschuss für Sozialschutz (Social Protection Committee) \\
SVRKAiG & Sachverständigenrat für die konzertierte Aktion im Gesundheitswesen \\
& seit Januar 2004 umbenannt in Sachverständigenrat zur Begutachtung \\
TK & der Entwicklung im Gesundheitswesen \\
u.a. & Techniker Krankenkasse \\
u.a.m. & unter anderem \\
u.U. & und anderem mehr \\
USA & unter Umständen \\
USt & United States of America \\
VdAK & Umsatzsteuer \\
VFA & Verband der Angestellten-Krankenkassen \\
VfG & Verband Forschender Arzneimittelhersteller \\
vgl. & Versandhaus für Gesundheit (tschechische Versandapotheke) \\
WDR & vergleiche \\
WHO & Westdeutscher Rundfunk \\
WIdO & World Health Organization \\
z.B. & Wissenschaftliches Institut der Ortskrankenkassen \\
z.T. & zum Beispiel \\
& zum Teil \\
& \\
\hline
\end{tabular}





\section{Einleitung}

Lange Zeit galten die Sozialversicherungssysteme und damit auch die Gesundheitssysteme der europäischen Mitgliedstaaten als Bereiche, die mit europäischem Recht nicht in Berührung kommen. Diese Einschätzung bekräftigte die in Art. 129 des Vertrags zur Gründung der Europäischen Gemeinschaft (EGV) (alt)' kodifizierte Beschränkung der Europäischen Gemeinschaft auf den Schutz der öffentlichen Gesundheit und die Koordinierung der Politiken der Mitgliedstaaten in diesem Bereich. Zwei Urteile des Europäischen Gerichtshofes (EuGH) in den Rechtssachen Kohll und Decker im Jahre 1998 widersprachen erstmals dieser Auffassung. ${ }^{2}$ Der Gerichtshof entschied in diesen beiden Urteilen, dass die Kostenübernahme im Fall einer Inanspruchnahme von Gesundheitsleistungen in einem anderen Mitgliedstaat aufgrund der im EGV verankerten Freiheiten des Waren- und Dienstleistungsverkehrs grundsätzlich nicht von einer vorherigen Genehmigung des zuständigen Finanzierungsträgers abhängig gemacht werden dürfe, solange keine gewichtigen Gründe eine Beschränkung dieser Freiheiten rechtfertigen. Der Tenor dieser Urteile war von Sozialrechtlern vielfach erwartet worden; die deutsche Gesundheitspolitik nahm dagegen zunächst eine abwehrende Haltung ein. Zum einen stellte sie die Gültigkeit der Urteile für das deutsche Gesundheitswesen in Frage, da es - anders als das in den Urteilen direkt betroffene luxemburgische System, das generell die Kostenerstattung vorsieht - auf dem Sachleistungsprinzip basiert. Zum anderen warnte der damalige Gesundheitsminister Seehofer für den Fall, dass auch für deutsche Patienten eine grenzüberschreitende Inanspruchnahme im Sinne der Kohll/Decker-Urteile möglich wäre, vor dem Verlust der finanziellen Steuerungsfähigkeit des Gesundheitswesens sowie einer ungewollten Harmonisierung der europäischen Gesundheitssysteme und einer Sozialunion auf niedrigstem Niveau. ${ }^{3}$ Die Urteile und die kontroversen Reaktionen von Seiten der Politik und Wissenschaft gaben die Anregung zum Thema dieser Arbeit, die Aufschluss darüber geben soll, auf welche Weise und in welchen Bereichen die Integration der europäischen Staaten sowie die EU als politische Institution direkt und indirekt Einfluss auf die Gesundheitspolitik der Mitgliedstaaten und speziell auf die Ausgestaltung des deutschen Gesundheitssystems nimmt.

Das Gesundheitswesen ist in vielen Ländern der Welt einer rein marktlichen Koordination entzogen. Ein staatlicher Eingriff kann aus ökonomischer Sicht gerechtfertigt werden, wenn den gehandelten Gütern mindestens eine der Eigenschaften fehlt, die Voraussetzungen für den ersten Hauptsatz der Wohlfahrtstheorie sind. Für eine Vielzahl von Gesundheitsgütern gilt in dieser Hinsicht, dass auf den entsprechenden Märkten aufgrund unvollkommener Informationen weder Markttransparenz noch Konsumentensouverănităt herrschen. Zudem treten bei einigen Gütern (z.B. Impfungen) externe Effekte auf.

\footnotetext{
' Durch den Vertrag von Amsterdam änderte sich die Nummerierung der Artikel des EGV. Da es im Rahmen dieser Arbeit notwendig ist, Artikel nach der alten und der neuen Nummerierung zu zitieren, wird zur Unterscheidung im ersten Fall der Zusatz (alt) hinzugefugt.

${ }^{2}$ Auf Einzelheiten dieser Urteile wird im Abschnitt 2.3.3.1 eingegangen.

${ }^{3}$ Vgl. o.V. (1998b), S. 36ff.
} 
Hinzu kommt, dass die Nachfrage nach Gesundheitsgütern zeitlich weitgehend zufällig erfolgt und u.U. mit extrem hohen Kosten verbunden ist, die die finanzielle Leistungsfähigkeit des Einzelnen übersteigen. Darum ist es für ein risikoaverses Individuum rational, sich gegen dieses Risiko zu versichern. Aufgrund von Anreizen zum Trittbrettfahrerverhalten ist jedoch davon auszugehen, dass bei einer rein privatwirtschaftlichen Organisation der Krankenversicherungsmärkte im Vergleich zur optimalen Allokation weniger Verträge nachgefragt würden. Zudem tragen vorhandene Informationsasymmetrien dazu bei, dass der Abschluss eines Krankenversicherungsvertrags nicht für alle Individuen möglich ist. Andererseits führt eine Versicherung, die sämtliche Krankheitskosten abdeckt, zu einer Verhaltensänderung der Versicherten gegenüber der Situation ohne Versicherung dahingehend, dass der Versicherte zu wenig zur Vermeidung des Schadens unternimmt bzw. dass im Versicherungsfall mehr Leistungen nachgefragt werden, als aus Effizienzgesichtspunkten wünschenswert wäre. Diese Tendenz kann u.U. durch das Verhalten der Ärzte verstärkt werden, wenn vom gewählten Vergütungssystem entsprechende unerwünschte Anreize ausgehen. Bei der Wahl des Vergütungssystems existiert grundsätzlich die Schwierigkeit, sowohl Anreize zu einer wirtschaftlichen Bereitstellung als auch zu einer qualitativ hochwertigen Versorgung zu generieren, ohne die Ärzte zu einer Auswahl ihrer Patienten nach deren Krankheitsrisiko (sog. Risikoselektion) zu veranlassen.

Fasst man das Ergebnis dieser Argumentation zusammen, so wird klar, dass das Gesundheitswesen - bestehend aus den Märkten für Gesundheitsgüter und Versicherungen gegen das Kostenrisiko bei Krankheit - nicht ohne einen angemessenen ordnungspolitischen Rahmen funktionieren kann. Ziel einer solchen staatlichen Ordnungspolitik sollte es sein, ein im Vergleich zur Marktlösung „besseres“ Ergebnis zu erreichen. Die Schwierigkeit bei der Gestaltung des Gesundheitswesens besteht jedoch darin, anreizkompatible Rahmenbedingungen zu setzen, die gleichzeitig die wesentlichen Prinzipien der gesetzlichen Krankenversicherung in Deutschland erhalten, wie z.B. die solidarische Finanzierung, einen Zugang zur gesetzlichen Krankenversicherung (GKV) für die gesamte Bevölkerung und die Umverteilung zwischen Personen mit hohem und niedrigem Krankheitsrisiko.

Im Hinblick auf die derzeitige Ausgestaltung der institutionellen Rahmenbedingungen des deutschen Gesundheitswesens ist es fraglich, ob diese dazu geeignet ist, das im Hinblick auf die Ziele Wirtschaftlichkeit und Qualität bestmögliche Ergebnis hervorzubringen. Vielmehr ist davon auszugehen, dass Ineffektivitäten und Ineffizienzen existieren, die dazu führen können, dass eine für die Krankheit des Patienten unangemessene Behandlung gewählt wird bzw. die Maßnahmen einen unnötig hohen Ressourceneinsatz erfordern. Als Gründe für Ineffektivitäten und Ineffizienzen sind z.B. zu nennen: ${ }^{4}$

- keine oder zu geringe Anreize für eine effektive und effiziente sektorenübergreifende Versorgung,

- zu geringe Transparenz über Behandlungsabläufe und Leistungsqualität,

${ }^{4}$ Vgl. Wille (2002), S. 5 f. 
- fehlende Wahlmöglichkeiten für die Versicherten und Patienten,

- mangelnde Patientenautonomie und fehlende Eigenverantwortung,

- Defizit in der Gesundheitsförderung und Prävention sowie in der Zertifizierung von Gesundheitsleistungen,

- ein Mangel an effektiver Qualitätssicherung und evidenzbasierten Behandlungsleitlinien,

- eine zu geringe Wettbewerbsintensität durch fehlende Handlungsparameter auf Seiten der Krankenkassen, damit einhergehend eine zu große Bedeutung des Beitragssatzes,

- das Fehlen eines "kassenspezifischen, zielorientierten Gesundheitsmanagements",

- zu wenige Werbemöglichkeiten für die Leistungserbringer,

- fehlende Orientierung der Vergütungssysteme an den Outcomes.

Die genannten Gründe sind grundsätzlich darauf zurückzuführen, dass die zahlreichen Regulierungen im Gesundheitswesen eine Anreizstruktur bilden, die dem Ziel einer wirtschaftlichen Erbringung qualitativ hochwertiger Leistungen oftmals entgegensteht. Im Mittelpunkt der Diskussionen über Veränderungen im deutschen Gesundheitssystem stehen darum neben der Umgestaltung des Leistungskatalogs sowie Veränderungen der Finanzierung und Beitragsgestaltung auch strukturelle Reformen zur Ausschöpfung von Wirtschaftlichkeitsreserven. Dies soll durch den Abbau von Regulierungen und dem Aufbau wettbewerblicher Strukturen zur Verbesserung von Versorgungsqualität und -effizienz erreicht werden.

Auch wenn diese Forderungen auf allgemeine Zustimmung stoßen, so ist bei der konkreten Umsetzung entsprechender Maßnahmen mit dem Widerstand derjenigen Gruppen zu rechnen, die aufgrund der Veränderungen eine ökonomische Verschlechterung ihrer Position hinnehmen müssten. Darum wurden die EuGH-Urteile aus dem Jahr 1998 von vielen in der Erwartung begrüßt, dass die Öffnung der nationalen Gesundheitsmärkte eine Veränderung der Strukturen erzwingen und damit - quasi durch die Hintertür - zu mehr Wettbewerb im System führen würde. ${ }^{5}$

In den vergangenen Jahren hat der EuGH diese Entscheidungen durch weitere Urteile präzisiert und erweitert. ${ }^{6}$ Die Entwicklung seither hat gezeigt, dass dramatische Effekte auf das deutsche Gesundheitswesen ausgeblieben sind. Inwieweit sich jedoch Veränderungen und Einflüsse ergeben haben, welche Entwicklungen in Zukunft zu erwarten sind und wie diese aus ökonomischer Sicht zu bewerten sind, ist Thema der vorliegenden Arbeit. Dabei finden neben den Urteilen des Gerichtshofs weitere Maßnahmen der Europäischen Institutionen Beachtung, da die Europäische Integration explizit und implizit auf verschiedenen Wegen Wirkung auf das deutsche Gesundheitssystem entfaltet.

${ }^{5}$ Vgl. z.B. Schulte (2000), S. 33.

${ }^{6}$ Vgl. zur Darstellung dieser Rechtsprechung Abschnitt 2.3.3.1. 
Das nachfolgende zweite Kapitel beinhaltet neben einem Abriss zur bisherigen Kompetenzentwicklung der Europäischen Union im Gesundheitsbereich eine Darstellung der wichtigsten Entwicklungen, durch welche die Europäische Integration auf die nationalen Gesundheitssysteme wirkt. Das dritte Kapitel analysiert darauf aufbauend die Auswirkungen der Europäischen Union auf wichtige Sektoren des deutschen Gesundheitswesens. Dabei werden weitere Maßnahmen und Entwicklungen auf europäischer Ebene berücksichtigt, die den jeweiligen Sektor isoliert betreffen und darum im allgemein gehaltenen zweiten Kapitel keinen Platz fanden. Im abschließenden Fazit erfolgen eine Zusammenführung der im dritten Kapitel gewonnenen Ergebnisse, eine Einschätzung der zu erwartenden Entwicklungen sowie deren Bewertung aus ökonomischer Sicht.

Trotz des derzeitigen Stillstands bei der Ratifizierung des Verfassungsentwurfs bleibt die Integration der europäischen Staaten ein dynamischer Prozess, wie die Diskussionen um einen EU-Beitritt der Türkei und die sog. Dienstleistungsrichtlinie zeigen. Die Beschäftigung mit den Auswirkungen der Europäischen Integration steht daher letztlich vor dem Problem, keine abschließende auch in Zukunft gültige Bewertung vornehmen zu können. Sie stellt gewissermaßen eine Momentaufnahme dar und benötigt die exogene Vorgabe eines Endzeitpunkts, bis zu dem die aktuellen Entwicklungen einbezogen werden können. Für diese Arbeit gilt der 31.07.2006 als solcher Endzeitpunkt. Entwicklungen, die sich nach diesem Datum ereignen, müssen darum bei dieser Untersuchung außen vor bleiben. 


\section{Wirkungen der Europäischen Integration im Gesundheitsbereich}

\subsection{Entwicklung der Kompetenzen der Europäischen Union}

Seit Beginn der europäischen Integration entfalteten Maßnahmen der Europäischen Gemeinschaften (EG) ${ }^{\top}$ Auswirkungen auf die nationalen Gesundheitssysteme. Die entsprechenden Gesetzes- und Rechtsakte konnten aber aufgrund der eingeschränkten Kompetenzen der EG im Bereich des Gesundheitswesens keine einheitliche europäische Gesundheitspolitik vorsehen. Die Idee einer Harmonisierung der Sozialsysteme diskutierte man zwar zu Beginn des europäischen Einigungsprozesses im „Europa der sechs"8 im Wesentlichen im Hinblick auf die Sicherstellung der Freizügigkeit von Arbeitnehmern, gab sie jedoch bald aufgrund der praktischen Schwierigkeiten auf, historisch gewachsene und sehr heterogene Systeme umzugestalten. ${ }^{9}$ Dennoch war die Politik der EG von Anbeginn an für die nationalen Gesundheitssysteme von Bedeutung. Wismar konnte bis zum 1. Halbjahr 1998245 Gesetzes- und Rechtsakte identifizieren, die Einfluss auf die Gesundheitspolitik der Mitgliedstaaten nahmen. ${ }^{10}$ Sie dienten im Wesentlichen zum Abbau von Handelshemmnissen und Wettbewerbsbeschränkungen oder stellten Maßnahmen zur Arbeitsplatzsicherheit dar.

Insgesamt lässt sich die Entwicklung der Kompetenzen der Gemeinschaft im Bereich der Gesundheitspolitik bis heute in fünf Phasen aufteilen. " Die erste Phase begann mit der Unterzeichnung des Vertrages über die Gründung der Europäischen Gemeinschaft für Kohle und Stahl (EGKS) im April 1951 und umfasst auch die Unterzeichnung der beiden anderen Gründungsverträge ${ }^{12}$. Wie bereits erwähnt, bestand die vorrangige Zielsetzung zunächst in der wirtschaftspolitischen Konsolidierung. Die Gesundheitspolitik stellte während dieser Zeit keinen eigenständigen Zuständigkeitsbereich dar, es gab aber bereits einzelne Maßnahmen mit Gesundheitsbezug (z.B. Gesundheitsschutz bei der Arbeit, Grundnormen für den Gesundheitsschutz der Bevölkerung und der Arbeitskräfte gegen ionisierende Strahlungen).

\footnotetext{
${ }^{7}$ Die Europäische Union (EU) besteht genau genommen erst seit dem Inkraftreten des Maastrichter Vertrags am 1.11.1993. Der politische Integrationsprozess begann im Jahr 1951 mit der Europäischen Gemeinschaft für Kohle und Stahl (EGKS) und wurde durch die Unterzeichnung der Römischen Verträge über die Gründung der Europäischen Wirtschaftsgemeinschaft (EWG) und der Europäischen Atomgemeinschaft (EAG bzw. Euratom) vertieft. EGKS, EWG und Euratom wurden auch als Europäische Gemeinschaften (EG) bezeichnet. Die EWG (durch den Maastrichter Vertrags umbenannt in „Europäische Gemeinschaft" (EG)) bildet seit 1993 mit der Gemeinsamen Außen- und Sicherheitspolitik (GASP) sowie der polizeilichen und justiziellen Zusammenarbeit im Strafrecht (PJZS) die drei Săulen der Europäischen Union. Innerhalb der folgenden Kapitel wird an den entsprechenden Stellen die Bezeichnung gewăhlt, die zum jeweiligen Zeitpunkt gültig war.

${ }^{8}$ Die sechs Gründungsmitglieder der Europäischen Gemeinschaften sind Frankreich, Deutschland, Italien, Luxemburg, Belgien und die Niederlande.

${ }^{9} \mathrm{Vgl}$. Schmucker (2003b), S. 3.

${ }^{10} \mathrm{Vgl}$. Wismar (1998), S. 33f.

" Vgl. zur Darstellung der ersten vier Phasen Schwanenflügel (1996), S. 10ff. und Hanika (1998), S. $193 f$.

12 Diese beiden Verträge werden als Verträge von Rom bezeichnet: Vertrag über die Europäische Wirtschaftsgemeinschaft und Vertrag über die Europäische Atomgemeinschaft, unterzeichnet am 25.3.1957, in Kraft getreten am 1.1.1958.
} 
Mit den Bemühungen um den Krankenversicherungsschutz für Wanderarbeitnehmer begann Anfang der 70er Jahre die zweite Phase der Kompetenzentwicklung. Die EWG-Verordnungen 1408/71/EWG und 574/72/EWG dienten zur Sicherstellung der Mobilität der Arbeitnehmer innerhalb der EG und damit zur Umsetzung der Niederlassungsfreiheit. ${ }^{13}$ Ebenfalls in diese Phase der Integration fielen Maßnahmen zum Gesundheitsschutz am Arbeitsplatz. Zudem wurden die ersten programmatischen Aktionen und Maßnahmenbündel implementiert, wie z.B. die Programme „Europa gegen Krebs" und „Europa gegen AIDS“. 1984 fand das erste informelle Treffen des Gesundheitsministerrates ${ }^{14}$ statt.

Als Startpunkt der dritten Phase kann die Unterzeichnung der Einheitlichen Europäischen Akte im Jahre 1986 ausgemacht werden. Sie ergänzte den EWG-Vertrag von 1957 u.a. um Art. 100a Abs. 3: „Die Kommission geht in ihren Vorschlägen nach Absatz 1 in den Bereichen Gesundheit, Sicherheit, Umweltschutz und Verbraucherschutz von einem hohen Schutzniveau aus." Durch die Einfügung dieses Artikels bekamen viele Richtlinien zur Verwirklichung des Binnenmarktes einen gesundheitspolitischen Bezug. Zu nennen sind hier beispielsweise die gemeinschaftsrechtliche Einflussnahme auf die Arzneimittelproduktion oder das Lebensmittelrecht sowie die Artikel 118a, $130 \mathrm{a}$ und $130 \mathrm{r} .{ }^{15} \mathrm{Ziel}$ war jedoch weiterhin vor allem die Verbesserung von wirtschaftlichen Vorgängen, d.h. der Abbau von Handelshemmnissen und die Harmonisierung von Normen, um Märkte zu integrieren und Wachstum und Wettbewerb zu fördern. ${ }^{16}$ In dieser Phase existierte noch keine unmittelbare und selbständige Kompetenz der EG im Bereich der Gesundheitspolitik. ${ }^{17}$

Die vierte Phase begann mit der Unterzeichnung des Maastrichter Vertrages im Jahre 1992 und wurde durch das Inkrafttreten des Amsterdamer Vertrages ${ }^{18}$ im Jahre 1999 vertieft. Mit dem Vertrag von Maastricht erhielt die Gesundheitspolitik der EU eine neue Rechtsgrundlage. Mit der Aufnahme eines eigenen Titels X „Gesundheitswesen“

\footnotetext{
${ }^{13} \mathrm{Vgl}$. Verordnung 1408/71/EWG des Rates vom 14. Juni 1971 über die Anwendung des Systems der sozialen Sicherheit auf Arbeitnehmer und Selbständige sowie deren Familienangehörige, die innerhalb der Gemeinschaft zu- und abwandern, in: ABl. EG Nr. L 149 v. 5.7.1971, S. 2-50; Verordnung 574/72/EWG des Rates vom 21. März 1972 über die Durchfuhrung der Verordnung (EWG) Nr. 1408/71 zur Anwendung der Systeme der sozialen Sicherheit auf Arbeitnehmer und deren Familien, die innerhalb der Gemeinschaft zu- und abwandern, in: ABl. EG Nr. L 74 vom 27.03.1972, S. 1-83.

Diese Verordnungen werden durch die Verordnung 883/2004/EG und die zugehörige Durchführungsverordnung aufgehoben werden, welche ab dem Tag gelten, an dem die Durchfuhrungsverordnung in Kraft tritt. Derzeit erarbeitet die Kommission einen Vorschlag zu dieser Verordnung.

${ }^{14}$ Rat der Gesundheitsminister der EU-Mitgliedstaaten.

${ }^{15}$ Diese Artikel haben folgende Inhalte:

118a: Verbesserung der Arbeitsumwelt,

130a: Stärkung des wirtschaftlichen und sozialen Zusammenhalts der Gemeinschaft, Verringerung der Unterschiede im Entwicklungsstand der verschiedenen Regionen und des Rückstands der am stärksten benachteiligten Gebiete,

130r: Umweltpolitik.

${ }^{16} \mathrm{Vgl}$. Schwanenflügel (1996), S. 12.

${ }^{17}$ Vgl. Hanika (1998), S. 194.

${ }^{18}$ Der Vertrag von Nizza, der am i.2.2003 in Kraft trat, ließ den Titel „Gesundheitswesen“ inhaltlich unverăndert und brachte damit keine Veränderungen für die Kompetenzen der EU mit sich.
} 
(im Sinne von Public Health ${ }^{19}$ ) erlangte die Gemeinschaft in Art. 129 EGV (alt) eigenständige Kompetenzen im Bereich der Gesundheitspolitik. ${ }^{20}$ Damit wurde der Gesundheitsbereich anderen Zielen gleichgestellt und die Einflussmöglichkeiten gemeinschaftlicher Gesundheitspolitik gegenüber anderen Politikbereichen gestärkt. Die Gesundheitspolitik war nun kein bloßer Annex anderer Politikbereiche mehr. Der Schwerpunkt der Kompetenzen der EU liegt seit dem Maastrichter Vertrag im Bereich der Prävention und umfasst damit auch Maßnahmen, die das Konsum- und Freizeitverhalten, den Sport- und den Erziehungsbereich berühren. ${ }^{21}$ Die Möglichkeit der EU zur Beeinflussung der Arbeit der Mitgliedstaaten in diesen Bereichen verstärkte sich dadurch deutlich. Neben den Grundlagen für die Gemeinschaftskompetenzen im Gesundheitswesen enthielt Artikel 129 EGV (alt) aber auch deren nachdrückliche Einschränkungen:22 Gesundheitspolitische Maßnahmen mit explizitem Bezug auf die Ausgestaltung der nationalen Gesundheitssysteme wurden als alleinige Aufgabe der Mitgliedstaaten betont und eine Harmonisierung der nationalen gesundheitspolitischen Gesetze und Regulierungen ausdrücklich ausgeschlossen (Harmonisierungsverbot).

Der Amsterdamer Vertrag (1999 in Kraft getreten) und auch der Vertrag von Nizza (2003 in Kraft getreten) ließen die vorrangigen Themenbereiche des Maastrichter Vertrages unberührt. So liegen die Hauptaufgaben der Europäischen Union im Bereich der Gesundheit weiterhin in der Prävention und der Sicherstellung eines hohen Gesundheitsschutzniveaus. Der präventive Charakter der ursprünglichen Formulierung wurde im Wortlaut noch verstärkt. ${ }^{23}$ Demnach ist es Aufgabe der Gemeinschaft, in Ergänzung der Politiken der Mitgliedstaaten die Gesundheit der Bevölkerung zu verbessern, Humankrankheiten zu verhüten und die Ursachen für die Gefährdung der menschlichen Gesundheit zu beseitigen. ${ }^{24}$ Der Vertrag betont, dass das Ziel der Sicherstellung eines hohen Gesundheitsschutzniveaus bei der Festlegung und Durchführung aller Gemeinschaftspolitiken sichergestellt werden muss. Dieser Querschnittscharakter der Gesundheitspolitik ist zwar nicht neu - schon der durch die Einheitliche Europäische Akte ergänzte Art. 100a EWG-Vertrag enthielt eine ähnliche Formulierung - er wurde jedoch durch Art. $152 \mathrm{EGV}^{25}$ noch einmal deutlich verstärkt, so dass der Gesundheitsschutz seither eine verpflichtenden Querschnittsaufgabe für alle Politikbereiche darstellt. ${ }^{26}$

19 „.,Public Health' [...] ist die interdisziplinäre Wissenschaft und Praxis der Gesunderhaltung von Bevölkerungsgruppen. Sie verfolgt das Ziel, Bedingungen zu schaffen und sicherzustellen, in denen Menschen gesund leben können. Schwerpunkt ist die Entwicklung und Umsetzung von Systemen der Gesundheitsförderung, der Krankheitsverhütung und Krankheitsbekămpfung bei effizientem Mitteleinsatz." Allgemeine Ortskrankenkasse (AOK) (2005f).

${ }^{20} \mathrm{Vgl}$. Schwanenflügel (1996), S. $64 f$.

${ }^{21}$ Vgl. Sachverständigenrat für die Konzertierte Aktion im Gesundheitswesen (SVRKAiG) (1992), S. 154.

${ }^{22}$ Vgl. Art. 129 Abs. 4 EGV.

${ }^{23} \mathrm{Vgl}$. Hollmann/Schulz-Weiden (1998), S. 184.

${ }^{24}$ Vgl. Art. 152, Abs. 1 EGV.

${ }^{25}$ Die Artikel des EGV wurden durch den Amsterdamer Vertrag neu nummeriert (vgl. auch Fn. 1). Der für das Gesundheitswesen wesentliche Artikel 129 des Maastrichter Vertrages trăgt seitdem die Nummer 152.

${ }^{26} \mathrm{Vgl}$. Schmucker (2003b), S. 9. 
Darüber hinaus enthält der Amsterdamer Vertrag in verschiedenen anderen Artikeln Bestimmungen mit Relevanz für den Bereich Gesundheit, die zwar bereits im Maastrichter Vertrag enthalten sind, deren Bedeutung für den Gesundheitsbereich z.T. aber mit dem Amsterdamer Vertrag verstärkt bzw. erst nach in Kraftreten des Vertrags deutlich wurde. Dazu zählen u.a.:"7

- Artikel 43-48 (Niederlassungsrecht; betrifft unter anderem Ärzte und andere Heilberufe),

- Artikel 49 und 50 (Dienstleistungen, einschließlich medizinischer und anderer gesundheitlicher Dienstleistungen),

- Artikel 95 (Angleichung der Rechtsvorschriften, einschließlich Lebensmittelsicherheit, Tabak, Arzneimittel, Medizinprodukte, Chemikalien und andere gefährliche Stoffe, Anwendungen der Biotechnologie),

- Artikel 131-133 (Gemeinsame Handelspolitik, z. B. bei Lebens- und Arzneimitteln),

- Artikel 137 (Soziale Sicherheit und sozialer Schutz der Arbeitnehmer),

- Artikel 149 (allgemeine und berufliche Bildung, einschließlich des Austauschs im Gesundheitssektor).

Damit ist es der Gemeinschaft möglich, zur Erreichung ihrer vorrangigen Ziele wie dem Schutz des Wettbewerbs und der Verwirklichung des Binnenmarktes Maßnahmen mit gesundheitspolitischem Inhalt zu ergreifen bzw. in Bezug auf andere Gemeinschaftsziele Regelungen zu treffen, die auch Auswirkungen auf die Funktionsweise der nationalen Gesundheitssysteme haben. ${ }^{28}$ Weiterhin bleibt aber der Ausschluss der Harmonisierung der nationalen Gesundheitspolitiken bestehen, der schon im Maastrichter Vertrag enthalten war.

Die fünfte Phase der Kompetenzentwicklung der EU im Bereich der Gesundheitspolitik, die heute noch andauert, begann mit der Frühjahrstagung des Europäischen Rates im Jahr 2000 in Lissabon. Im Rahmen der sog. Lissabonner Strategie, die das Ziel hat, „die Union zum wettbewerbsfähigsten und dynamischsten wissensbasierten Wirtschaftsraum der Welt zu machen“, beschloss der Rat, die sog. „offene Methode der Koordinierung" (OMK), die zuvor bereits bei der Umsetzung der Maastricht-Kriterien zur Währungsunion erfolgreich eingesetzt wurde, auf den Bereich der sozialen Sicherheit auszudehnen. Ihre beschränkten Kompetenzen in diesem Bereich machen der EU eine direkte Einflussnahme auf die Politiken der Mitgliedstaaten unmöglich. Die Anwendung der OMK ist darum für solche Politikbereiche vorgesehen, in denen die Gemeinschaft lediglich über begrenzte Befugnisse verfügt. ${ }^{29}$ Im Gesundheitsbereich erfolgt der Einsatz der Methode, um die Mitgliedstaaten in der Gewährleistung ihres Sozialschutzes und der Modernisierung ihrer Sozialsysteme zu unterstützen, wie es in Einklang mit Art. 137 und 140 EGV Aufgabe der EU ist. Die OMK kann als Versuch

\footnotetext{
${ }^{27}$ Vgl. Europäische Kommission (2000a), S. 26.

${ }^{28}$ Vgl. Berg (1997), S. 449.

${ }^{29} \mathrm{Vgl}$. Europäische Kommission (2003d), S. 10.
} 
verstanden werden, die gemeinschaftliche Kompetenzverteilung in diesen Gebieten und damit auch bei der Gesundheitspolitik - zu modifizieren. Eine detaillierte Darstellung der Methode, ihrer Ziele sowie des derzeitigen Stands der Umsetzung erfolgt in Abschnitt 2.5.

Bevor in Abschnitt 2.3 auf die Bedeutung der Grundfreiheiten des EGV für das Gesundheitssystem und insbesondere auf die Rechtsprechung des EuGH in Bezug auf Gesundheitsleistungen eingegangen wird, gibt der folgende Abschnitt einen kurzen Überblick über die Aktionsprogramme der Europäischen Kommission im Bereich des Gesundheitsschutzes, die sie auf Basis ihrer Kompetenzen seit 1993 auflegte.

\subsection{Aktionsprogramme im Bereich der öffentlichen Gesundheit}

Zur Umsetzung der im Maastrichter Vertrag verankerten Ziele „Prävention“ und „Erreichung eines hohen Gesundheitsschutzniveaus“ veröffentlichte die EG-Kommission im November 1993 eine Mitteilung über einen Aktionsplan im Bereich der öffentlichen Gesundheit. ${ }^{30}$ Darin betonte die Kommission, dass die EG ihre Arbeit in diesem Bereich unter Beachtung der vier Grundsätze Subsidiarität, Offenheit, Transparenz und Proportionalität ausüben wolle. Die Kommission erarbeitete in der Mitteilung Kriterien, anhand derer entschieden werden sollte, welche Maßnahmen im Bereich der offfentlichen Gesundheit auf Gemeinschaftsebene zu ergreifen sind. Auf Basis dieser Kriterien schlug sie acht Aktionsprogramme vor, die in den darauf folgenden Jahren umgesetzt wurden:

- Aktionsprogramm der Gemeinschaft zur Gesundheitsförderung, -aufklärung, -erziehung und -ausbildung,

- Aktionsplan zur Krebsbekämpfung,

- Aktionsprogramm der Gemeinschaft zur Prävention von Aids und bestimmten anderen übertragbaren Krankheiten,

- Aktionsprogramm der Gemeinschaft zur Suchtprävention,

- Aktionsprogramm der Gemeinschaft zur Gesundheitsberichterstattung,

- Aktionsprogramm der Gemeinschaft zur Verhütung von Verletzungen,

- Aktionsprogramm der Gemeinschaft betreffend seltene Krankheiten,

- Aktionsprogramm der Gemeinschaft betreffend durch Umweltverschmutzung bedingte Krankheiten.

Die EG stellte zur Finanzierung dieser Programme 300 Mio. $€$ zur Verfügung, wobei der Schwerpunkt auf den Programmen zur Bekämpfung von Krebs (64 Mio. $€$ ) und Aids (60 Mio. $€$ ) lag. ${ }^{31}$ Neben diesen Aktionsprogrammen befasste sich die dafür zuständige Generaldirektion für Gesundheit und Verbraucherschutz mit weiteren Maß-

\footnotetext{
${ }^{30} \mathrm{Vgl}$. Europäische Kommission (1993).

${ }^{31}$ Vgl. Weingärtner (2000), S. 33f.
} 
nahmen im Bereich der Gesundheitspolitik wie beispielsweise mit der Erarbeitung von Strategien zur Bekämpfung des Tabakkonsums oder den Jahresberichten über die Themen „Gesundheitszustand in der Gemeinschaft" und „Integration der Gesundheitsschutzerfordernisse in andere Politiken “ ${ }^{32}$ Alle diese Maßnahmen ließen die nationalen Gesundheitspolitiken nicht unberührt, so dass trotz des im Vertrag von Maastricht verankerten Harmonisierungsausschlusses von einem wachsenden Einfluss der EG in diesem Bereich gesprochen werden kann, der jedoch (noch) nicht in die Organisation der nationalen Gesundheitssysteme hereinreichte.

Nach der Ratifizierung des Amsterdamer Vertrags untersuchte die Kommission, ob der bestehende Aktionsrahmen ausreichte, um auf die zahlreichen wichtigen Entwicklungen, wie beispielsweise auf neu entstehende Gesundheitsgefährdungen, steigenden Kostendruck im Gesundheitswesen, die Erweiterung der Gemeinschaft und die neuen Bestimmungen über die öffentliche Gesundheit im Amsterdamer Vertrag, reagieren zu können. ${ }^{33}$ Sie kam zu dem Schluss, dass die Grundsätze und die politische Richtung der Mitteilung über den Aktionsrahmen im Bereich der öffentlichen Gesundheit von 1993 weiterhin Gültigkeit besaßen, die Prioritäten, Strukturen und Methoden jedoch einer grundsätzlichen Überprüfung und Neuformulierung bedurften.

Die vorherigen Aktionsprogramme sahen sich Kritik ausgesetzt, da sie mit ihrer groBen Zahl und dem "vertikalen" Ansatz, für jedes Problem ein eigenes Programm aufzulegen, zu unübersichtlich und ineffizient seien. ${ }^{34}$ Die Kommission schlug daraufhin für die zukünftige Politik drei "horizontale" Aktionsschwerpunkte vor, die sie im Jahre 2000 in ihren Vorschlag für eine neue "Gesundheitspolitische Strategie der Europäischen Gemeinschaft" aufnahm: ${ }^{35}$

- Verbesserung der Gesundheitsinformation,

- Einführung eines Schnellreaktionssystems bei Gefahren für die öffentliche Gesundheit,

- Berücksichtigung der für die Gesundheit bestimmenden Faktoren.

Den zentralen Ausgangspunkt für die strategische Neuausrichtung der Kommission stellte die Betonung des Querschnittscharakters des Gesundheitsschutzes im Amsterdamer Vertrag dar. ${ }^{36}$

Die Kommission hatte das neue Aktionsprogramm ursprünglich für die Jahre 2001 bis 2006 geplant, die Verabschiedung verzögerte sich jedoch wegen Meinungsverschiedenheiten der zuständigen Gremien. ${ }^{37}$ Der Plan stieß im Europäischen Parlament auf Widerstand, da darin eine unzulässige Ausweitung der Kompetenzen der EU im Bereich der Gesundheitspolitik gesehen wurde, die laut EGV ausdrücklich den Mitglied-

\footnotetext{
${ }^{32} \mathrm{Vgl}$. auch zu weiteren Maßnahmen Europäische Kommission (2000a), S. $26 \mathrm{f}$.

${ }^{33} \mathrm{Vgl}$. Europäische Kommission (1998).

${ }^{34} \mathrm{Vgl}$. Schmucker (2003b), S. 14.

${ }^{35} \mathrm{Vgl}$. Europäische Kommission (2000a), S. $2 \mathrm{f}$.

${ }^{36} \mathrm{Vgl}$. Europäische Kommission (2000a), S. 3.

${ }^{37} \mathrm{Vgl}$. Schmucker (2003b), S. 15.
} 
staaten vorbehalten bleiben. ${ }^{38}$ Schließlich erfolgte die Verabschiedung des neuen Aktionsplans durch das Europäischen Parlament und den Rat im September 2002; er gilt nun für die Jahre 2003 bis $2008 .{ }^{39}$ Die Umsetzung des Planes verfolgt als Hauptziel die Ergänzung der Politiken der Mitgliedstaaten, die dem Schutz der menschlichen Gesundheit dienen oder auf Verbesserungen im Gesundheitswesen abzielen. ${ }^{40}$ Entsprechend dem Kommissionsvorschlag gelten als allgemeine Ziele:

- die Verbesserung des Informations- und Wissensstandes im Interesse der Weiterentwicklung des öffentlichen Gesundheitswesens,

- die Verbesserung der Fähigkeit zur schnellen und koordinierten Reaktion auf Gesundheitsgefahren,

- die Gesundheitsförderung und Verhütung von Krankheiten durch Berücksichtigung gesundheitsrelevanter Faktoren in allen Politiken und Tätigkeiten.

Das Programm soll durch die Förderung einer integrierten und sektorenübergreifenden Gesundheitsstrategie dazu beitragen, bei der Festlegung und Durchführung sämtlicher Gemeinschaftspolitiken und -maßnahmen ein hohes Niveau des Gesundheitsschutzes sicherzustellen. Zudem sollen Ungleichheiten im Gesundheitsbereich abgebaut und die Zusammenarbeit zwischen den Mitgliedstaaten in den in Art. 152 EGV aufgefuhrten Bereichen gefördert werden.

Die Kommission plant derzeit ein neues Aktionsprogramm für die Jahre 2007 bis 2013, das die Bereiche Gesundheit und Verbraucherschutz integrieren soll, um die Synergien aus den Einzelstrategien zu nutzen. ${ }^{4}$ Laufende Programme sollen im Aktionsplan verschmelzen und um fünf Schwerpunkte ergänzt werden, wozu auch die Verbesserung der Effizienz und der Effektivität der Gesundheitssysteme zählt. Das Aktionsprogramm würde die Europäische Kommission dazu legitimieren, sich verstärkt in den Themenbereichen „grenzüberschreitende Inanspruchnahme von Gesundheitsleistungen“, „Informationsaustausch über Gesundheitsfragen“, „Förderung der Patientensicherheit" sowie "Schaffung eines europäischen Netzes von Referenzzentren" engagieren zu können. ${ }^{42}$

Der Überblick über die von der Kommission seit 1993 aufgelegten und vorgeschlagenen Aktionsprogramme zeigt deutlich, dass sie den Willen hat, ihre durch den EGV verliehenen Kompetenzen im Bereich Prävention und Gesundheitsschutz zu nutzen. Damit nimmt sie Einfluss auf verschiedene Politikfelder der Mitgliedstaaten. Darüber hinaus lässt sich auch im Bereich der Organisation der Gesundheitssysteme, der entsprechend dem Subsidiaritätsprinzip in die alleinige Zuständigkeit der nationalen Regierungen fällt, der Versuch einer wachsenden Einflussnahme erkennen, was - wie die Diskussion um das aktuelle Aktionsprogramm zur öffentlichen Gesundheit zeigte - in den Mitgliedstaaten auf Kritik stößt. Darum ist zu erwarten, dass das derzeit in Pla-

\footnotetext{
${ }^{38} \mathrm{Vgl}$. Friedrich (2002).

${ }^{39} \mathrm{Vgl}$. Europăisches Parlament und Rat (2002), Art. 1.

${ }^{40} \mathrm{Vgl}$. im Folgenden Europäisches Parlament und Rat (2002), Art. 2.

${ }^{41} \mathrm{Vgl}$. Europäische Kommission (2005d).

${ }^{42}$ Vgl. o.V. (2005c), S. A 1629.
} 
nung befindliche neue Programm vor seiner Umsetzung erhebliche politische Hürden überwinden muss.

Der Bedeutungszuwachs der Gesundheitspolitik auf europäischer Ebene steht in engem Zusammenhang mit den Urteilen des EuGH zur grenzüberschreitenden Inanspruchnahme von Gesundheitsleistungen, da erst durch die Möglichkeit einer größeren Patientenwanderung der Wunsch nach einer stärkeren Koordinierung der Gesundheitssysteme entstanden ist. Das folgende Kapitel gibt einen Überblick über die Bedeutung der vier Grundfreiheiten des EGV im Gesundheitswesen und erläutert dabei auch die Inhalte der relevanten EuGH-Entscheidungen.

\subsection{Bedeutung der europäischen Grundfreiheiten im Gesundheits- wesen}

Die vier Grundfreiheiten des EGV setzen sich zusammen aus den sachbezogenen Freiheiten des Waren- und Kapitalverkehrs sowie den personenbezogenen Freiheiten des Dienstleistungs- und Personenverkehrs. ${ }^{43}$ Ihre Umsetzung ist seit Beginn der Europäischen Integration von großer Bedeutung für die Verwirklichung eines gemeinschaftsweiten Binnenmarktes. Der EGV garantiert dabei keine einheitliche und umfassende wirtschaftliche Freiheit, sondern regelt die verschiedenen Freiheiten in unterschiedlichen Abschnitten mit einzelnen Rechtsvorschriften. Durch diese gesonderten Regelungen fanden die einzelnen Freiheiten in unterschiedlicher Breite und Tiefe Anwendung, wobei die Entwicklung der Rechtsetzung auf europäischer Ebene und insbesondere die Rechtsprechung des EuGH zu einer Konvergenz der Grundfreiheiten hin zu einer allgemeinen wirtschaftlichen Betätigungsfreiheit beitragen ${ }^{44}$ Diese Entwicklung lässt sich auch hinsichtlich der Anwendung der verschiedenen Freiheiten auf die Systeme der sozialen Sicherung und damit bezüglich des Gesundheitswesens beobachten. Während die Gewährleistung der Personenverkehrsfreiheit seit Beginn der Integrationsbemühungen auch auf Regelungen des Gesundheitswesens Auswirkungen hatte, ging man bis zu den EuGH-Urteilen in den Rechtssachen Kohll und Decker im Jahr 1998 davon aus, dass die Waren- und die Dienstleistungsfreiheit für die im Rahmen des Gesundheitssystems erbrachten Leistungen ohne Bedeutung seien. Die Freiheit des Kapitalverkehrs spielt für die deutsche Sozialversicherung und insbesondere für die gesetzliche Krankenversicherung eine untergeordnete Rolle ${ }^{45}$ und ist im Rahmen dieser Arbeit lediglich insoweit interessant, als sie Tendenzen zur Kettenbildung und Konzentration beispielsweise in der Arzneimittelindustrie verstärkt bzw. ermöglicht. ${ }^{46}$

\footnotetext{
${ }^{43}$ Vgl. Art. 3 Abs. 1c EGV.

${ }^{44} \mathrm{Vgl}$. Schulte (1999a).

${ }^{45}$ So auch Schaub (2001), S. 44.

${ }^{46} \mathrm{Da}$ aber für die Umsetzung der Kapitalverkehrsfreiheit im Gesundheitswesen keine speziellen Regelungen und Maßnahmen notwendig sind, wird auf eine Darstellung der Freiheit in diesem Kapitel verzichtet.
} 


\subsubsection{Freiheit des Personenverkehrs}

\subsubsection{Niederlassungsfreiheit}

Die Freiheit des Personenverkehrs kommt zum einen in der Niederlassungsfreiheit für Selbständige, zum anderen in der Arbeitnehmerfreizügigkeit zum Ausdruck. Im Rahmen des Gesundheitswesens ist die Niederlassungsfreiheit für die Mitglieder derjenigen Berufe relevant, die in selbständiger Tätigkeit ausgeübt werden - hierzu zählen beispielsweise niedergelassene Ärzte und Apotheker. Für sie besteht aufgrund dieser Freiheit die Möglichkeit, ihrer Tätigkeit dauerhaft in einem anderen EU-Mitgliedstaat nachzugehen. ${ }^{47}$ Die entsprechenden Regelungen finden sich in den Art. 43ff. EGV und stellen damit - ebenso wie die anderen Grundfreiheiten - primäres Gemeinschaftsrecht dar. ${ }^{48}$

Die Niederlassungsfreiheit umfasst die Aufnahme und Ausübung selbständiger Erwerbstätigkeit sowie die Gründung und Leitung von Unternehmen ${ }^{49}$ und gilt auch gegenüber dem Staat, dessen Staatsangehörigkeit man besitzt, wenn es sich um die Beurteilung eines Sachverhaltes handelt, der das Merkmal einer Grenzüberschreitung aufweist. ${ }^{50}$ So entschied der EuGH in der Rechtssache „Broekmeulen“51, dass ein niederländischer Arzt, der in Belgien sein Staatsexamen erhalten hatte, in den Niederlanden zur ärztlichen Tătigkeit zugelassen werden muss, obwohl er die in der niederländischen Zulassungsordnung geforderte praktische Vorbereitungszeit nicht abgeleistet hatte. Relevant ist hier u.a., dass der Kläger gegenüber Personen aus anderen EUMitgliedstaaten, die ohne Ableistung dieser Vorbereitungszeit zugelassen wurden, nicht benachteiligt werden darf. Auf Fälle, in denen inländische Ärzte durch die Umsetzung der Niederlassungsfreiheit schlechter gestellt werden als ihre ausländischen Kollegen, ist das Europarecht jedoch nicht anwendbar. Darum findet die Schlechterstellung der Inländer im genannten Urteil keine Beachtung und ist nach nationalem Recht zu beurteilen..$^{52}$ Eine solche Inländerdiskriminierung entsteht, wenn die Mitgliedstaaten gemeinschaftsrechtliche Vergünstigungen nicht auch zugunsten ihrer Staatsangehörigen wirken lassen wollen. Eine nationale Zulassungsbeschränkung ist darum - soweit sie inländische Ärzte mit im Inland erworbenen Examina betrifft - alleine nach nationalem Recht zu prüfen. ${ }^{53}$ Ein Beispiel hierfür ist die im System der deutschen GKV praktizierte Bedarfsplanung, die bewirkt, dass sich Ärzte in als „überversorgt" eingestuften Regionen (sog. Planungsbereichen) nur im Rahmen einer Praxisnachfolge oder einer Sonderbedarfszulassung ${ }^{54}$ niederlassen können.

\footnotetext{
${ }^{47}$ Eine vorübergehende Tătigkeit in einem anderen Mitgliedstaat der EU fällt dagegen unter die (aktive) Dienstleistungsfreiheit (Art. 49ff EGV).

${ }^{48}$ Zum primären Gemeinschaftsrecht zählen die Regelungen der Gründungsverträge der Europäischen Gemeinschaft sowie alle Anhänge und Ergănzungen dieser Vertrăge. Vgl. Krőck (1998), S. 21.

${ }^{49} \mathrm{Vgl}$. Art. 43 EGV.

${ }^{50}$ Vgl. Kröck (1998), S. 22 sowie die dort angegebene Rechtsprechung des EuGH.

s1 Vgl. Europäischer Gerichtshof (1981).

52 Vgl. Kröck (1998), S. 31 und Weis (1983), S. 2721.

${ }^{53}$ Vgl. Kröck (1998), S. 30.

${ }^{54}$ Eine Sonderbedarfszulassung ist zu erteilen, wenn mindestens eine der folgenden Bedingungen gilt:
} 
Um eine möglichst umfangreiche Niederlassungsfreiheit für Ärzte zu gewährleisten, legte die Kommission bereits 1969 entsprechende Richtlinienvorschläge vor. Diese wurden in der Folgezeit kontrovers diskutiert. Der zügige Erlass scheiterte an den groBen Unterschieden innerhalb der Rechtsordnungen der Mitgliedstaaten und wurde durch den Beitritt von Dänemark, Irland und England zur EG am 1. Januar 1973 zusätzlich erschwert. ${ }^{55}$ Der EuGH entschied im Jahre 1974 jedoch, dass die Niederlassungsfreiheit unmittelbar geltendes Recht darstellt ${ }^{56}$, so dass ihre Gültigkeit nicht vom Erlass der in Artikel 54 und 57 EWGV vorgeschriebenen Richtlinien abhängt, und jeder Unionsbürger sich auch gegenüber nationalen Gerichten auf diese - und die anderen - Grundfreiheiten berufen kann, wenn es sich um die Beurteilung eines entsprechenden grenzüberschreitenden Sachverhalt handelt. ${ }^{57}$ Das Urteil förderte den Integrationsprozess im Bereich der Niederlassungsfreiheit nachhaltig, so dass am 20. Juni 1975 die Richtlinie 75/362/EWG zur gegenseitigen Anerkennung der Diplome, Prüfungszeugnisse und sonstigen Befähigungsnachweise für Ärzte sowie die zugehörige Koordinierungsrichtlinie 75/363/EWG in Kraft traten. Im Jahre 1986 erfolgte darüber hinaus durch die Richtlinie 86/457/EWG die Einführung einer europaweit vergleichbaren, spezifischen Ausbildung in der Allgemeinmedizin..$^{58}$ Die Richtlinie 93/16/EWG fasste diese Richtlinien im Jahre $1993 \mathrm{zu}$ einem einzigen Normtext zusammen. Entsprechend gilt für pharmazeutische Diplome seit 1985 die Richtlinie 85/433/EWG über die gegenseitige Anerkennung der Diplome, Prüfungszeugnisse und sonstigen Befähigungsnachweise des Apothekers und über Maßnahmen zur Erleichterung der tatsächlichen Ausübung des Niederlassungsrechts für bestimmte pharmazeutische Tätigkeiten. ${ }^{59}$

Es stellt sich nun aus europarechtlicher Sicht die Frage, ob nationale Zulassungsbeschränkungen mit der europäischen Niederlassungsfreiheit vereinbar sind, d.h. ob diese Grundfreiheit des EGV u.U. eine Beschränkung der Niederlassung von ausländischen Ärzten verbietet. Dies würde bedeuten, dass beispielsweise die in Deutschland

\footnotetext{
Es existiert nachweislich lokaler Versorgungsbedarf in der vertragsärztlichen Versorgung in Teilen eines großstädtischen Planungsbereich oder eines großräumigen Landkreises.

Es ist ein besonderer Versorgungsbedarf in speziellen fachlichen Teilbereichen vorhanden. Dieser Versorgungsbedarf muss durch den Inhalt eines Schwerpunkts, einer fakultativen Weiterbildung oder einer besonderen Fachkunde für das Facharztgebiet nach der Weiterbildungsordnung umschrieben sein. Die Zulassung wird dabei auf das Leistungsspektrum beschrănkt, für das im entsprechenden Planungsbereich Versorgungsbedarf besteht.

Es gibt einen Mangel an spezialisierten Gemeinschaftspraxen mit spezialisierten Versorgungsaufgaben.

Es besteht ein Mangel an Praxen mit dem Schwerpunkt ambulantes Operieren.

Vgl. Gemeinsamer Bundesausschluss (2006), Nr. 24 Satz 1 a bis d.

55 Vgl. Kröck (1998), S. 58.

${ }^{56} \mathrm{Vgl}$. Europäischer Gerichtshof (1974b); für die unmittelbare Gültigkeit der Dienstleistungsfreiheit vgl. Europäischer Gerichtshof (1974a), Rn. 24 und 26.

${ }^{57}$ Vgl. Kröck (1998), S. 23; Schirmer (2003), S. 152.

${ }^{58}$ Vgl. Kröck (1998), S. 59.

${ }^{59}$ Die genannten Richtlinien werden ihre Gültigkeit am 20.10.2007 mit dem Inkrafttreten der Richtlinie 2005/36/EG verlieren. Diese Richtlinie fasst 15 bisher sektorale Richtlinien zusammen und vereinheitlicht und vereinfacht die Regeln über die Anerkennung von erworbenen Qualifikationen.
} 
im Bereich der vertragärztlichen Versorgung durchgeführte Bedarfsplanung nicht auf Ärzte mit ausländischen Abschlüssen angewandt werden könnte. Diese Frage ist bisher nicht abschließend beantwortet und wird in der Literatur unterschiedlich bewertet. $^{60}$ So wird zum einen argumentiert, dass Art. $43 \mathrm{EGV}$, der eine Beschränkung der Niederlassung von Bürgern eines anderen EU-Mitgliedstaates verbietet, auf Zulassungsbeschränkungen nicht anwendbar sei, solange ausländische und inländische Ärzte gleichermaßen betroffen seien, wenn also keine Diskriminierung der ausländischen Ärzte vorliege. Zum anderen existiert jedoch die Auffassung, dass es sich bei der Niederlassungsfreiheit um ein allgemeines Grundrecht der EU-Bürger handele und sie damit Zulassungsbeschränkungen für Ärzte aus anderen Mitgliedstaaten generell verbiete, unabhängig davon, ob inländische Mediziner ebenfalls an der Berufsausübung gehindert seien.$^{61}$ Kurz gesagt, handelt es sich aus rechtlicher Sicht um die Frage, ob sich aus der Niederlassungsfreiheit lediglich ein Diskriminierungsverbot oder ein weitergehendes Beschränkungsverbot ergibt. Aus zwei Urteilen aus den Jahren 1993 und 1995 kann gefolgert werden, dass auch nicht-diskriminierende Beschränkungen einem Rechtfertigungsdruck unterliegen und der EuGH Art. 43 EGV - wie die anderen Grundfreiheiten auch - als Beschränkungsverbot interpretiert. ${ }^{62}$ In der Rechtssache „Kraus" stellte der Gerichtshof klar, dass Art. 39 und 43 EGV, jeder nationalen Regelung [entgegenstehen], die zwar ohne Diskriminierung aus Gründen der Staatsangehörigkeit anwendbar ist, die aber geeignet ist, die Ausübung der [...] grundlegenden Freiheiten durch die Gemeinschaftsangehörigen einschließlich der Staatsangehörigen des Mitgliedstaats, die die Regelung erlassen hat, zu behindern oder weniger attraktiv zu machen." ${ }^{63}$ In der Rechtssache „Gebhard“ verweist der Gerichtshof auf dieses Urteil und führt zudem aus, welche Voraussetzungen Regelungen eines Mitgliedstaates, die eine Ausübung der Grundfreiheiten des EGV verhindern oder weniger attraktiv machen, erfüllen müssen, um nicht gegen europäisches Recht zu verstoßen: „Sie müssen in nicht-diskriminierender Weise angewandt werden, sie müssen aus zwingenden Gründen des Allgemeinwohls gerechtfertigt sein, sie müssen geeignet sein, die Verwirklichung des mit ihnen verfolgten Ziels zu gewährleisten, und sie dürfen nicht über das hinausgehen, was zur Erreichung des Zieles erforderlich ist."64

Grundsätzlich ist bei einer Interpretation der Rechtsprechung des EuGH Vorsicht angebracht. ${ }^{65}$ Der Gerichtshof entscheidet generell auf den jeweiligen Einzelfall bezogen und scheint die Reichweite seiner Urteile nicht immer zu berücksichtigen, so dass es nur eingeschränkt möglich ist, aus bereits ergangenen Urteilen verallgemeinernde Schlüsse $\mathrm{zu}$ ziehen. Zudem ist in der betrachteten Frage eine Bewertung nur auf Grundlage der für Ärzte tatsächlich einschlägigen Urteile möglich, da die Berücksich-

\footnotetext{
${ }^{60} \mathrm{Vgl}$. Kröck (1998), S. 78ff und die dort angegebene Literatur.

${ }^{61}$ Vgl. z.B. Pitschas (1994), S. 108f; Hănlein (1993), S. 190ff.

${ }^{62}$ Vgl. Kröck (1998), S. 87.

${ }^{63}$ Europäischer Gerichtshof (1993a), Rn. 32.

${ }^{64}$ Europäischer Gerichtshof (1995), Rn. 39.

${ }^{65} \mathrm{Vgl}$. Kröck (1998), S. 88.
} 
tigung von Entscheidungsgründen aus Urteilen, die Angehörige anderer freier Berufe ${ }^{66}$ betreffen, zu Verwirrungen führen kann. In Bezug auf die deutsche Bedarfsplanung steht die europarechtliche Bewertung durch den EuGH darum noch aus. Da dort derzeit keine entsprechende Rechtssache anhängig ist und zudem vom Eingang einer Rechtssache bis zur Entscheidung des Gerichtshofs in aller Regel mehrere Jahr vergehen, ist zu dieser Frage auf kurze Sicht keine Klärung durch den EuGH zu erwarten. So ist eine Niederlassung ausländischer Ärzte im Rahmen der vertragsärztlichen Versorgung momentan nur entsprechend den Regelungen zur Bedarfsplanung möglich.

\subsubsection{Arbeitnehmerfreizügigkeit}

Bereits zu Beginn der europäischen Integrationsbemühungen wurde berücksichtigt, dass die Wahrnehmung des Rechts auf Freizügigkeit eng mit der Koordinierung der sozialen Sicherungssysteme der einzelnen Mitgliedstaaten verknüpft ist. Der Vertrag zur Gründung der Europäischen Wirtschaftsgemeinschaft (EWGV) von 1957 räumte dem europäischen Rat das Recht ein, „die auf dem Gebiet der sozialen Sicherheit für die Herstellung der Freizügigkeit der Arbeitnehmer notwendigen Maßnahmen ${ }^{467}$ zu beschließen. Entsprechende Verordnungen (Nr. 3 und 4) erließ der Rat bereits im Jahre 1958, worin die Bedeutung des europäischen koordinierenden Sozialrechts für die Funktionsfähigkeit einer echten Freizügigkeit deutlich wird. ${ }^{68}$ Diese beiden Verordnungen wurden in den 70er Jahren durch die Verordnung 1408/71/EWG und die dazugehörige Durchführungsverordnung 571/72/EWG abgelöst, die bis heute gelten. Das sog. freizügigkeitsspezifische Sozialrecht stellt keine Harmonisierung im Bereich der sozialen Sicherheit dar, sondern koordiniert lediglich die einzelstaatlichen Maßnahmen auf diesem Gebiet. ${ }^{69}$ Die Kompetenz zur Organisation ihrer sozialen Sicherungssysteme bleibt weiterhin bei den Mitgliedstaaten. Jeder Mitgliedstaat kann darum selbst darüber entscheiden, wer nach seinen Rechtsvorschriften versichert ist und welche Leistungen unter welchen Bedingungen gezahlt werden. Die Regelungen der Verordnung 1408/71/EWG sollen aber garantieren, dass eine Person, die von ihrem Recht auf Freizügigkeit Gebrauch macht, nicht schlechter gestellt wird als eine Person, die ihr Leben lang im selben Mitgliedstaat gewohnt und gearbeitet hat. Die Verordnung galt zunächst nur für Arbeitnehmer und ihre Familienangehörigen, wurde später aber auch

\footnotetext{
${ }^{66}$ Nach der Definition des Bundesverbands der Freien Berufe (BFB) zăhlen diejenigen Berufe zu den freien Berufen, deren Angehörige ,auf Grund besonderer beruflicher Qualifikation persönlich, eigenverantwortlich und fachlich unabhängig geistig-ideelle Leistungen im gemeinsamen Interesse ihrer Auftraggeber und der Allgemeinheit [erbringen]. Ihre Berufsausubung unterliegt in der Regel spezifischen berufsrechtlichen Bindungen nach Maßgabe der staatlichen Gesetzgebung oder des von der jeweiligen Berufsvertretung autonom gesetzten Rechts, welches die Professionalität, Qualität und das zum Auftraggeber bestehende Vertrauensverhältnis gewăhrleistet und fortentwickelt." Bundesverband der Freien Berufe (BFB) (2006). Zu diesen freien Berufen zăhlen laut Einkommensteuergesetz u.a. Ärzte, Zahnärzte, Rechtsanwälte, Notare, Architekten und Journalisten, vgl. § 18 Abs. 1 Nr. 1 S. 2 EStG.

${ }^{67}$ Art. 51 EWGV.

${ }^{68}$ So auch Schaub (2001), S. 44.

${ }^{69} \mathrm{Vgl}$. Europäische Kommission (2002), S. 11.
} 
auf Selbständige und deren Familienmitglieder ${ }^{70}$ sowie auf Studierende ${ }^{71}$ ausgedehnt. ${ }^{72}$ Seit dem Jahr 1998 sind auch Sondersysteme für Beamte in die Verordnung mit einbezogen. Die Bestimmungen gelten in den Ländern der EU sowie in Island, Liechtenstein und Norwegen - und damit im gesamten Europäischen Wirtschaftsraum (EWR) - und seit 2002 in der Schweiz. Aufgrund der Verordnung 859/2003/EG können sich schließlich auch Drittstaatenangehörige und ihre Familienmitglieder auf diese Verordnung berufen, soweit sie ihren rechtmäßigen Wohnsitz in einem Mitgliedstaat haben.

Die Verordnung 1408/71/EWG dient zur Koordinierung der Rechtsvorschriften in den Zweigen der sozialen Sicherheit, die Leistungen infolge von Krankheit und Mutterschaft, Invalidität, Alter, Arbeitsunfällen und Berufskrankheiten, Arbeitslosigkeit und Tod sowie Familienleistungen betreffen. ${ }^{73}$ So regelt sie auch die Versicherungszugehörigkeit von EU-Bürgern, die in einem anderen Mitgliedstaat als ihrem Wohnsitzstaat abhängig beschäftigt sind (sog. Wanderarbeitnehmer). Darüber hinaus haben Personen, die sich vorübergehend in einem anderen Mitgliedstaat aufhalten oder wohnen, im Krankheitsfall auf Kosten ihres zuständigen Versicherungsträgers Anspruch auf Sachleistungen gemäß den Rechtsvorschriften des ausländischen Mitgliedstaates, als ob sie dort versichert wären. ${ }^{74}$ Wenn ein Versicherter sich gezielt zu einer Behandlung ins Ausland ${ }^{75}$ begibt, hat er Anspruch auf eine Kostenübernahme durch sein Versicherungsland, wenn er zuvor eine Genehmigung für die Behandlung erhalten hat. ${ }^{76}$ Diese Genehmigung darf nicht verweigert werden, wenn die betreffende Behandlung zu den im System des Wohnortes vorgesehenen Leistungen gehört und er die Behandlung in Anbetracht seines Gesundheitszustandes und des voraussichtlichen Verlaufs der Krankheit nicht in einem Zeitraum erhalten kann, der für diese Behandlung in seinem Wohnortstaat normalerweise erforderlich ist, " d.h. wenn die Erbringung der Leistung nach den im Versicherungsstaat üblichen Maßstäben nicht rechtzeitig möglich ist. ${ }^{78}$ Eine Inanspruchnahme von Gesundheitsleistungen im Rahmen der Verordnung 1408/71/EWG ist damit auf drei Fälle beschränkt: ${ }^{79}$

- eine unverzüglich notwendige Behandlung während eines Auslandsaufenthalts (Notfall),

\footnotetext{
${ }^{70}$ Verordnung 3095/95/EWG vom 22.12.1995, ABI. L 335 vom 30.12.1995.

${ }^{71}$ Verordnung Nr. 307/1999/EG vom 8.2.1999, ABl. L 38 vom 12.2.1999.

${ }^{72} \mathrm{Vgl}$. Europäische Kommission (2002), S. 13.

${ }^{73} \mathrm{Vgl}$. Art. 4 Verordnung 1408/71/EWG.

${ }^{74} \mathrm{Vgl}$. Europäische Kommission (2002), S. 15.

75 Im Rahmen dieser Arbeit ist mit dem Begriff „Ausland“ stets das europäische Ausland (EWR und Schweiz) gemeint. Nur dort ist eine Leistungsinanspruchnahme zu Lasten der GKV entsprechend der hier vorgestellten Regelungen bzw. der in Abschnitt 2.3.3.1 dargestellten EuGH-Rechtsprechung möglich.

${ }^{76} \mathrm{Vgl}$. Art. 22 Abs. 1 lit c) Verordnung 1408/71/EWG. Auf diese Möglichkeit der grenzüberschreitenden Inanspruchnahme von Gesundheitsleistungen wird in Abschnitt 3.2.1 genauer eingegangen.

${ }^{77}$ Vgl. Art. 22 Abs. 2 Satz 2 Verordnung 1408/71/EWG.

${ }^{78} \mathrm{Vgl}$. Fuchs (2005), Rn. 61

${ }^{79} \mathrm{Vgl}$. Schaub (2001), S. 48.
} 
- bei vorheriger Genehmigung des zuständigen Trägers im Fall eines Wohnortwechsels und

- bei vorheriger Genehmigung im Fall einer gewünschten Auslandsbehandlung mit einem Rechtsanspruch auf diese Genehmigung, wenn die oben genannten Bedingungen erfült sind.

Die Regelungen, die die Behandlungen in Notfällen und bei vorheriger Genehmigung betreffen ${ }^{80}$, gelten nicht nur für die in Art. 2 der Verordnung genannten Personengruppen $^{81}$, sondern beziehen alle krankenversicherten EU-Bürger sowie die bei ihnen wohnenden Familienangehörigen mit ein. ${ }^{82}$

Zahlreiche Veränderungen haben die Verordnung 1408/71/EWG sehr komplex werden lassen, weshalb die Kommission im Dezember 1998 Vorschläge für eine effizientere und nutzerfreundlichere Ausgestaltung unterbreitete. Am 29. April 2004 verabschiedeten der Rat und das Europäische Parlament die Verordnung 883/2004/EG zur „Koordinierung der Systeme der sozialen Sicherheit". Diese Verordnung enthält grundlegende Reformen und Vereinfachungen der Bestimmungen für die Koordinierung im Bereich der sozialen Sicherheit, um die soziale Absicherung der Bürger zu verbessern. Dabei lässt sie die Möglichkeiten zur Inanspruchnahme von Gesundheitsleistungen nach dem koordinierenden Sozialrecht weitgehend unverändert, passt aber die Regelungen an die betreffende Rechtsprechung des EuGH an ${ }^{83}$ So ändert sich beispielsweise die Voraussetzung für die Erteilung einer Genehmigung. In Art. 20 der Verordnung 883/2004/EG heißt es in diesem Zusammenhang: „Die Genehmigung wird erteilt, wenn die betreffende Behandlung Teil der Leistungen ist, die nach den Rechtsvorschriften des Wohnmitgliedstaats der betreffenden Person vorgesehen sind, und ihr diese Behandlung nicht innerhalb eines in Anbetracht ihres derzeitigen Gesundheitszustands und des voraussichtlichen Verlaufs ihrer Krankheit medizinisch vertretbaren Zeitraums gewährt werden kann." Die Verordnung 883/2004/EG stellt damit nicht mehr auf die „normale“ Bereitstellungszeit am Wohnort ab, sondern verwendet nur noch objektive Kriterien. Die Verordnung ersetzt die bestehende Verordnung 1408/71/EWG, sobald die erforderlichen Durchführungsvorschriften verabschiedet sind, d.h. wahrscheinlich nicht vor Ende des Jahres 2006. ${ }^{84}$

\subsubsection{Freiheit des Warenverkehrs}

Die Warenverkehrsfreiheit ist in Art. 28 und 29 EGV verankert, die mengenmäßige Ein- und Ausfuhrbeschränkungen sowie Maßnahmen gleicher Wirkung verbieten. Maßnahmen gleicher Wirkung sind nach der „Dassonville-Formel“ alle Handelsrege-

${ }^{80} \mathrm{Vgl}$. Art. 22 Abs. 1 lit. a) und c) Verordnung 1408/71/EWG.

${ }^{81}$ Laut Art. 2 Verordnung 1408/71/EWG gilt diese Verordnung für Arbeitnehmer, Selbständige und Studierende sowie für deren Familienangehörige und Hinterbliebene. ${ }^{82} \mathrm{Vgl}$. Art. 22a Verordnung 1408/71/EWG.

${ }^{83}$ Siehe zu einer ausführlichen Darstellung dieser Rechtsprechung Abschnitt 2.3.3.1.

${ }^{84} \mathrm{Vgl}$. Europäische Kommission (2005c). 
lungen der Mitgliedstaaten, die geeignet sind, den innergemeinschaftlichen Handel unmittelbar und mittelbar, tatsächlich oder potentiell zu behindern. ${ }^{85}$ Eine Einschränkung erfuhr die „Dassonville-Formel“ durch die Cassis-Rechtsprechung ${ }^{86}$, so dass Hemmnisse für den Binnenhandel der Gemeinschaft, die sich aus den Unterschieden der nationalen Regelungen bezüglich der Herstellung und Vermarktung von Produkten ergeben, hingenommen werden müssen, soweit diese Bestimmungen notwendig sind, um zwingenden Erfordernissen gerecht $\mathrm{zu}$ werden und es sich um nichtdiskriminierende Handelsregelungen handelt. Darüber hinaus erlaubt Art. 30 EGV Handelsbeschränkungen u.a. zum Schutz der Gesundheit und des Lebens von Menschen.

Im Bereich des Gesundheitswesens spielt die Warenverkehrsfreiheit insbesondere für den Arzneimittelmarkt eine große Rolle. In den vergangenen Jahren wurden auf der europäischen Ebene Maßnahmen erlassen, die den freien Arzneimittelverkehr in Europa stärken sollen. Hierzu zählt beispielsweise die Einführung eines seit 1995 europaweit geltenden Zulassungsverfahrens, das die Marktzulassung für Arzneimittel in der EU erleichtert. Zudem schützt die Warenverkehrsfreiheit entsprechend der Rechtsprechung des EuGH den Parallel- und Reimport von Arzneimitteln. Als Parallelimporte bezeichnet man Arzneimittel, die der Hersteller außerhalb des Empfängerlandes produziert und die von einem Parallelimporteur im Land der Produktion erworben und parallel zu den Vertriebswegen des Herstellers oder Erstlieferanten im Empfängerland auf den Markt gebracht werden. Bei reimportierten Arzneimitteln handelt es sich um in einem Land produzierte Medikamente, die vom Hersteller oder Erstlieferanten ins Ausland exportiert und dann von einem Parallelhändler ins Herstellerland zurück importiert werden. ${ }^{87}$ Innerhalb der EU bestehen Anreize zu Re- und Parallelimporten von Arzneimitteln, da sich aufgrund der nationalstaatlichen Zuständigkeit für die Erstattungs- und Preisregulierungen im Rahmen der gesetzlichen Gesundheitssysteme zwischen den Mitgliedstaaten z.T. erhebliche Preisdifferenzen für ein Produkt ergeben.

Die rechtliche Möglichkeit zum Parallelhandel ergibt sich aus dem Vorrang des freien Warenverkehrs vor dem Recht des Markeninhabers auf die alleinige Vermarktung des Produkts. ${ }^{88}$ Der Markeninhaber kann sich auf dieses Recht nicht mehr berufen, sobald das Arzneimittel in einem Staat der EU auf den Markt gebracht worden ist (Grundsatz der regionalen Erschöpfung). Eine vorübergehende Ausnahme stellen die neuen Mitgliedstaaten Tschechien, Estland, Lettland, Litauen, Ungarn, Polen, Slowenien und Slowakei dar: Der Markeninhaber kann einen Parallelimport aus diesen Ländern verhindern, wenn das Patent auf ein Produkt zu einem Zeitpunkt erlassen wurde, zu dem

\footnotetext{
${ }^{85}$ Vgl. Europäischer Gerichtshof (1974c), Leitsatz 1.

${ }^{86} \mathrm{Vgl}$. Europäischer Gerichtshof (1979)

${ }^{87}$ In den Veröffentlichungen der EU wird zwischen diesen beiden Begriffen nicht unterschieden. Dort dienen die Begriffe „Parallelimporte“ und „Parallelhandel“ vielmehr als Oberbegriffe für die Einfuhr von Waren, die außerhalb der offiziellen Vertriebskanäle des Herstellers oder Lizenznehmers aus einem Land in ein anderes eingefuhrt und dort in Verkehr gebracht werden.

${ }^{88}$ Vgl. Danzon (1998), S. 296.
} 
es nicht möglich war, dort ein Patent oder ein ergänzendes Schutzzertifikat ${ }^{89} \mathrm{zu}$ erwerben. ${ }^{90}$ Für die übrige EU gilt jedoch, dass der Markeninhaber von seinem Markenrecht keinen Gebrauch machen darf, um das Umpacken eines parallel eingeführten Produktes zu verhindern, wenn:9!

- der Gebrauch des Markenrechts durch den Inhaber zu einer künstlichen Abschottung der Märkte zwischen den Mitgliedstaaten führt,

- das Umpacken den Originalzustand der Ware nicht beeinträchtigen kann,

- auf der neuen Verpackung angegeben ist, von wem das Produkt hergestellt und umverpackt wurde,

- das umgepackte Arzneimittel nicht so aufgemacht ist, dass dadurch der Ruf der Marke und ihres Inhaber geschädigt werden kann und

- der Markeninhaber unterrichtet wird, bevor das umgepackte Erzeugnis zum Kauf angeboten wird.

Der EuGH hat jedoch auch klargestellt, dass der Parallelimporteur ein Arzneimittel nur dann umpacken darf, wenn es objektiv notwendig ist. Das Umpacken ist entsprechend der Rechtsprechung des EuGH beispielsweise dann objektiv notwendig, wenn der Markeninhaber das gleiche Arzneimittel in unterschiedlichen Packungen und/oder unter einer anderen Marke in verschiedenen Mitgliedstaaten in den Verkehr gebracht hat und Packungen der im Exportland vertriebenen Größe im Einfuhrmitgliedstaat nicht vertrieben werden können. Diese Notwendigkeit ist aber nicht gegeben, wenn der Importeur das Umpacken, das Wiederanbringen der Marke oder ihre Ersetzung ausschließlich aus wirtschaftlichen Gründen vornehmen möchte.

Besitzt ein Arzneimittel eine nationale Zulassung in einem Mitgliedstaat, so kann der Parallelhändler für die Einfuhr in ein anderes Mitgliedsland die Möglichkeit eines im Vergleich zum Genehmigungsverfahren für die erstmalige Zulassung „vereinfachten“ Verfahrens nutzen, wenn das einzuführende Medikament im Wesentlichen einem gleicht, für das im Mitgliedstaat der Einfuhr bereits eine Genehmigung besteht. ${ }^{92} \mathrm{Im}$ hierzu grundlegenden Urteil vom 20. Mai 1976 entschied der EuGH in der Rechtssache „De Peijper ${ }^{603}$, dass es für den Gesundheitsschutz nicht erforderlich sei, bei einem Parallelimport dieselben Unterlagen wie für eine erstmalige Zulassung zu verlangen, wenn ein ,identisches“ Arzneimittel mit dem selben Ursprung in dem betroffenen Importland bereits eine Zulassung besitzt. ${ }^{94}$ Auf die Frage der Gleichheit zweier Produkte ging der EuGH in seiner Einscheidung vom 12. November 1996 in der Rechtssache

\footnotetext{
${ }^{89}$ Ein ergänzendes Schutzzertifikat für ein Arzneimittel kann nach dem Ablauf des Patents beantragt werden und verlängert den Patentschutz für das betreffende Medikament um maximal fünf Jahre. Die rechtliche Grundlage für dieses Zertifikat bildet die Verordnung 1768/92/EWG.

${ }^{90} \mathrm{Vgl}$. Europäische Kommission (2003e), S. 11f. Diese Ausnahme gilt nicht furr Malta und Zypern, da ein Patent oder ein ergänzender Schutz hier bereits vor deren Beitritt erlangt werden konnten.

${ }^{91} \mathrm{Vgl}$. im Folgende Europäische Kommission (2003e), S. 13f.

${ }^{92} \mathrm{Vgl}$. Europäische Kommission (2003e), S. 3 und 7.

${ }^{93} \mathrm{Vgl}$. Europäischer Gerichtshof (1976).

${ }^{94} \mathrm{Vgl}$. Koenig/Engelmann/Sander (2001), S. 921.
} 
„Smith \& Nephew Pharmaceuticals“ ein. Demnach müssen die zuständigen nationalen Behörden eine für ein Arzneimittel erteilte Genehmigung für ein weiteres Medikament gelten lassen, wenn die beiden Arzneispezialitäten, ohne in allen Punkten übereinzustimmen, zumindest nach der gleichen Formel und unter Verwendung des gleichen Wirkstoffs hergestellt worden sind und überdies die gleiche therapeutische Wirkung haben, soweit dem keine Erwägungen eines wirksamen Schutzes des Lebens und der Gesundheit von Menschen entgegenstehen. ${ }^{95}$ Bestehen jedoch therapeutische Unterschiede, so ist eine Genehmigung nach dem vereinfachten Verfahren nicht möglich, und der Parallelimporteur benötigt vor der Einfuhr eine neue Vollzulassung. ${ }^{96}$

Der EuGH hat mit Hinweis auf die Freiheit des Warenverkehrs in diesen und weiteren Urteilen, die die Verletzungen des Markenrechts, doppelte Preissysteme sowie Lieferbeschränkungen betrafen, immer wieder dafür gesorgt, dass die Möglichkeiten der nationalen Akteure, d.h. insbesondere der Originalhersteller, einen Re- oder Parallelimport ihrer Produkte zu verhindern, sehr begrenzt sind. Erst in jüngerer Zeit gab es Hinweise darauf, dass der EuGH beginnt, die Problematik der Beeinflussung von Forschungs- und Entwicklungsaktivitäten durch Parallelimporte sowie den Konflikt zwischen freiem Wettbewerb und staatlich regulierten Arzneimittelmärkten in seine Entscheidungen mit einzubeziehen. In den verbundenen Rechtssachen C-2/01 und C-3/01 „Bundesverband der Arzneimittel-Importeure gegen Kommission der Europäischen Gemeinschaften“ ist der EuGH der Entscheidung des Europäischen Gerichts erster Instanz gefolgt. Im Widerspruchsverfahren gegen das Verbot eines Kontingentierungssytems der Bayer AG hatte das Gericht bereits im Jahre 2000 festgestellt, dass ein Hersteller die Lieferungen an einen Großhändler auf die Mengen beschränken darf, die für die Versorgung des jeweiligen Landes notwendig sind, um auf diese Weise den Reimport seiner Produkte einzudämmen. Eine solche Begrenzung stellt demnach keine verbotene wettbewerbshindernde Vereinbarung zwischen Unternehmen nach Art. 81 EGV dar. Das Gericht hatte der Überzeugung der Kommission widersprochen, dass Parallelimporte langfristig zu einer Harmonisierung der Arzneimittelpreise führen würden" und die These zurückgewiesen, es gebe ein generelles „Verbot der Verhinderung von Parallelimporten, das nicht nur für die Mitgliedstaaten, sondern auch - und in

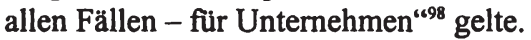

In eine ähnliche Richtung weist die Argumentation des Generalstaatsanwalts des EuGH Jacobs im Schlussantrag zur Rechtssache C-53/03 „Syfait und andere ${ }^{\text {“99 }}$. Der Arzneimittelhersteller Glaxo Wellcome hatte im November 2000 die Lieferung von Medikamenten an griechische Arzneimittelgroßhändler und Apothekergenossenschaften beschränkt, um Reimporte seiner Produkte zu begrenzen. Darum richtete die griechische Wettbewerbskommission ein Vorabentscheidungsersuchen an den EuGH. Der Generalstaatsanwalt vertrat in seinem Schlussantrag angesichts der wirtschaftlichen

\footnotetext{
${ }^{95} \mathrm{Vgl}$. Europäischer Gerichtshof (1996), Leitsatz 1.

${ }^{96} \mathrm{Vgl}$. Europäischer Gerichtshof (1996), Leitsatz 1; Koenig/Engelmann/Sander (2001), S. 922.

${ }^{97} \mathrm{Vgl}$. Europäisches Gericht erster Instanz (EuG) (2000), Rn. 181.

${ }^{98}$ Europäisches Gericht erster Instanz (EuG) (2000), Rn. 178

${ }^{99}$ Europäischer Gerichtshof (2004a).
} 
Besonderheiten der pharmazeutischen Industrie die Auffassung, dass eine Lieferbeschränkung eines marktbeherrschenden Pharmaunternehmens, die darauf abzielt, den Parallelhandel zu begrenzen, als eine angemessene und verhältnismäßige Maßnahme zum Schutze der geschäftlichen Interessen gerechtfertigt sein könne, da eine Lieferpflicht dem Hersteller den Anreiz zu innovativer Tätigkeit nehme und nicht zwingend Freizügigkeit und Wettbewerb fördere. Zudem sei nicht anzunehmen, dass der Parallelhandel den Endverbrauchern von Arzneimitteln oder den Mitgliedstaaten als deren Hauptabnehmer tatsächliche nütze. ${ }^{100}$ Der EuGH verwies die Entscheidung aus verfahrenstechnischen Gründen an die nationalen Gerichte zurück, so dass das mit Spannung erwartete Grundsatzurteil zur Frage, ob und unter welchen Umständen Pharmafirmen dazu berechtigt sind, die Belieferung von Großhändlern zu begrenzen, noch immer aussteht.

Nachdem die Europäische Kommission in Parallelimporten lange Zeit ein wichtiges Instrument sah, um den Wettbewerb im Arzneimittelmarkt zu stärken und das Ziel eines einheitlichen Binnenmarktes für Arzneimittel zu erreichen, ${ }^{101}$ könnte das Urteil zugunsten der Bayer AG mit dazu beigetragen haben, dass die Europäische Kommission beginnt, ihre bisherige Politik zu überdenken. Offenbar erörterte die Generaldirektion Wirtschaft in einem internen Reflexionspapier im Oktober 2004 die Frage, ob man sich statt auf die Erleichterung des Parallelimports nicht eher auf die Förderung des Generikawettbewerbs konzentrieren solle und damit die Angleichung der Preise bzw. Preisregulierungen der offenen Koordinierung zwischen den Mitgliedstaaten überlässt. ${ }^{102}$ Inwieweit dies tatsächlich zu einer veränderten Haltung der Kommission führt und welche konkreten Konsequenzen sich in Zukunft fur ihre Arzneimittelpolitik ergeben, bleibt abzuwarten.

Die private Einfuhr von Arzneimitteln zum persönlichen Gebrauch ist zulässig, wenn die Medikamente im Einfuhrmitgliedstaat zugelassen sind und aus einer Apotheke stammen, unabhängig davon, ob sie verschreibungspflichtig sind. Bei einer Verschreibungspflicht muss das Arzneimittel jedoch von einem Arzt innerhalb der EU verschrieben worden sein. ${ }^{103}$

In der Rechtssache „Decker“ beschäftigte sich der EuGH darüber hinaus mit der Frage, welche Bedeutung der Warenverkehrsfreiheit in Bezug auf eine grenzüberschreitende Nachfrage von Gesundheitsgütern zukommt. ${ }^{104}$ Die Argumentation zu diesem Urteil entspricht zum Teil wortgleich der Begründung des EuGH im Urteil zur Rechtssache „Kohll' ${ }^{\text {clos }}$, das am gleichen Tag ergangen ist und sich auf die Dienstleistungsfreiheit bezieht. Die Darstellung dieser beiden Urteile erfolgt darum gemeinsam in Abschnitt 2.3.3.1, der die Rechtsprechung des EuGH zur grenzüberschreitenden Inanspruchnahme von Gesundheitsleistungen darstellt.

\footnotetext{
${ }^{100} \mathrm{Vgl}$. Europäischer Gerichtshof (2004a), Rn. 100.

${ }^{101} \mathrm{Vgl}$. Hancher (2004).

${ }^{102} \mathrm{Vgl}$. Meyer (2005), S. 18.

${ }^{103}$ Vgl. Kröck (1998), S. 257.

${ }^{104} \mathrm{Vgl}$. Europäischer Gerichtshof (1998b).

${ }^{105} \mathrm{Vgl}$. Europäischer Gerichtshof (1998c).
} 


\subsubsection{Freiheit des Dienstleistungsverkehrs}

Die Dienstleistungsfreiheit schützt auf Grundlage der Art. 49 bis 55 EGV sowohl die aktive Erbringung von Dienstleistungen durch EU-Bürger in einem anderen Mitgliedstaat als auch die EU-weite grenzüberschreitende Nachfrage nach Dienstleistungen (passive Dienstleistungsfreiheit). Dienstleistungen im Sinne des EGV weisen folgende Merkmale auf: Es handelt sich um selbständige wirtschaftliche Tätigkeiten, die i.d.R. gegen Entgelt erbracht werden und deren Ausübung lediglich vorübergehend in einem anderen Mitgliedstaat erfolgt.

Die aktive Dienstleistungsfreiheit gibt natürlichen und juristischen Personen das Recht, in einem anderen Mitgliedstaat als ihrem Heimatstaat eine Dienstleistung zu den gleichen Bedingungen wie Inländer zu erbringen (Diskriminierungsverbot). ${ }^{106}$ Damit ergänzt sie die Niederlassungsfreiheit dahingehend, dass grenzüberschreitende erwerbswirtschaftliche Tätigkeiten auch ohne Wohnsitzverlagerung möglich sind. Sie umfasst auch das Recht der Leistungserbringer im Gesundheitswesen, ihre Tätigkeit vorübergehend in einem anderen Mitgliedstaat auszuüben, ohne dort niedergelassen zu sein. Diese Leistungserbringung wird jedoch durch Aspekte der sozialen Sicherheit erschwert, so dass Ärzte und viele andere Angehörige von Gesundheitsberufen kaum als „normale“ Dienstleister betrachtet werden können. ${ }^{107}$

Im nächsten Abschnitt erfolgt zunächst eine Darstellung der Rechtsprechung des EuGH zur passiven Dienstleistungsfreiheit, die mit dem Urteil in der Rechtssache „Kohll“" begann und in den darauf folgenden Jahren durch verschiedene Entscheidungen weiterentwickelt und präzisiert wurde.

\subsubsection{EuGH-Rechtsprechung zur passiven Dienstleistungsfreiheit}

Während bei der Nutzung der aktiven Dienstleistungsfreiheit der Leistungserbringer selbst in einem anderen Mitgliedstaat tätig wird, gibt die passive Dienstleistungsfreiheit den Konsumenten die Möglichkeit, Leistungen grenzüberschreitend nachzufragen, indem sie einen Anbieter in einem anderen Mitgliedstaat aufsuchen. Die im Folgenden dargestellte Rechtsprechung des EuGH beschäftigt sich in diesem Zusammenhang mit der Frage, inwieweit im Fall einer Inanspruchnahme von Gesundheitsleistungen im Ausland die Kostenübernahme von einer vorherigen Genehmigung des zuständigen Trägers abhängig gemacht werden darf, ohne dass es zu einer unzulässigen Verletzung der Dienstleistungsfreiheit kommt.

Die erste Entscheidung in dieser Frage traf der Gerichtshof im Jahre 1998 in der Rechtssache „Kohll“. Wie bereits erwähnt, erging ein ähnliches Urteil in Bezug auf die Freiheit des Warenverkehrs am gleichen Tag in der Rechtssache „Decker“, welches

\footnotetext{
${ }^{106}$ Vgl. Art. 49 Abs. 1 EGV.

${ }^{107}$ So auch Jorens/Coucheir/Overmeiren (2005), S. 22. In Abschnitt 2.3.3.2 wird darauf eingegangen, ob die derzeit bestehenden Möglichkeiten zur vorübergehenden grenzüberschreitenden Leistungserbringung ausreichen, um die Dienstleistungsfreiheit der Leistungserbringer umfassend zu gewăhrleisten.
} 
hier ebenfalls berücksichtigt wird. In den betreffenden Fällen hatten luxemburgische Sozialversicherte die Kostenübernahme für eine in Deutschland durchgeführte Zahnkorrektur bzw. eine in Belgien gekaufte Brille beantragt. Ihre Krankenkassen lehnten dies mit der Begründung ab, dass nach luxemburgischem Recht eine Inanspruchnahme von Leistungen im Ausland zu Lasten der Krankenkassen nur nach vorheriger Genehmigung erfolgen könne. Der EuGH hatte nun zu entscheiden, ob eine solche Regelung mit den Grundfreiheiten des EGV vereinbar ist. In seiner Entscheidung betonte der Gerichtshof, dass das Gemeinschaftsrecht die Kompetenzen der Mitgliedstaaten zur Ausgestaltung ihrer Systeme der sozialen Sicherheit zwar grundsätzlich unberührt lässt, ${ }^{108}$ sie aber gleichwohl bei der Ausübung dieser Befugnis das Gemeinschaftsrecht beachten müssen. ${ }^{109}$ Aus diesem Grund schließt auch die Zugehörigkeit der strittigen Regelungen zum Bereich der sozialen Sicherheit die Anwendung der entsprechenden Grundfreiheiten nicht aus. ${ }^{110} \mathrm{Da}$ das Genehmigungserfordernis die Sozialversicherten darin behindert, Waren und Dienstleistungen bei ausländischen Leistungserbringern nachzufragen, verstößt die entsprechende Regelung gegen diese Freiheiten."'

Allerdings kann ein Eingriff in die Grundfreiheiten aus einem der im EGV geregelten Rechtfertigungsgründe ${ }^{112}$ oder aus zwingenden Gründen des Gemeinwohls - z.B. einer erheblichen Gefährdung des finanziellen Gleichgewichts des Systems der sozialen Sicherheit - gerechtfertigt werden. Eine solche Gefährdung verneinte der Gerichtshof in den betrachteten Fällen jedoch mit dem Hinweis, dass eine Erstattung lediglich bis zur Höhe der Kosten zu erfolgen habe, wie sie bei einer Inanspruchnahme der Leistungen im Inland entstanden wären. Auch dem Einwand, ein Genehmigungsvorbehalt sei aus Gründen des Schutzes der öffentlichen Gesundheit notwendig, widersprach der EuGH, da die Bedingungen des Zugangs zu geregelten Berufen und ihrer Ausübung zahlreichen Harmonisierungsrichtlinien unterliegen und darum in anderen Mitgliedstaaten der EU tätige Leistungserbringer als ebenso qualifiziert anzuerkennen seien wie einheimische.

Diese Urteile kamen für Sozial- und Europarechtler nicht überraschend, riefen jedoch bei den politischen Entscheidungsträgern - nicht nur in Deutschland - ablehnende Reaktionen hervor. ${ }^{113}$ Der damalige deutsche Gesundheitsminister Horst Seehofer ging davon aus, dass die beiden Urteile keine Auswirkungen auf das deutsche Sozialrecht hätten, da die deutsche gesetzliche Krankenversicherung auf dem Sachleistungsprinzip basiert, während das von den Urteilen direkt betroffene luxemburgische Gesundheitswesen die Kostenerstattung vorsieht. Da der EuGH nur bezogen auf den jeweiligen Einzelfall urteilt, war diese Frage nach den Urteilen in den Rechtssachen „Kohll“ und „Decker" unbeantwortet geblieben. Darüber hinaus stellte sich die Frage, ob die Entscheidungen lediglich für den in den Urteilen betrachteten ambulanten Bereich oder

\footnotetext{
${ }^{108}$ Vgl. Europäischer Gerichtshof (1998c), Rn. 17; Europäischer Gerichtshof (1998b), Rn. 21.

${ }^{109} \mathrm{Vgl}$. Europäischer Gerichtshof (1998c), Rn. 19; Europäischer Gerichtshof (1998b), Rn. 26.

${ }^{110}$ Vgl. Europäischer Gerichtshof (1998c), Rn. 21; Europäischer Gerichtshof (1998b), Rn. 25.

III Vgl. Europäischer Gerichtshof (1998c), Rn. 35; Europäischer Gerichtshof (1998b), Rn. 36.

112 Hier kommt gemäß Art. 36 EGV bzw. Art. 66 i.V.m. Art. 56 EGV der Schutz der Volksgesundheit in Betracht. Vgl. Kötter (1999), S. 244.

${ }^{113} \mathrm{Vgl}$. zu den Reaktionen der Mitgliedstaaten auf die EuGH-Urteile Palm et al. (2000), S. 78ff.
} 
auch für Krankenhausleistungen Gültigkeit habe. In den darauf folgenden Jahren präzisierte der EuGH seine Rechtsprechung in den Entscheidungen zu den Rechtssachen „Smits und Peerbooms" ${ }^{\text {(114 }}$, „Vanbraekel“1/15, „Müller-Fauré und van Riet"(116 und "Watts“117.

Am 12.7.2001 entschied der EuGH in der Rechtssache "Smits und Peerbooms". Im ersten Fall hatte eine in den Niederlanden Sozialversicherte die Kostenerstattung für eine in Deutschland durchgeführte stationäre Behandlung ihrer Parkinsonschen Krankheit beantragt. Diese Erstattung wurde vom zuständigen Träger mit der Begründung abgelehnt, dass eine ausreichende und angemessene Behandlung in den Niederlanden verfügbar gewesen sei. In der Rechtssache Peerbooms wurde ein niederländischer Komapatient in Österreich mittels eines Verfahrens behandelt, dass in den Niederlanden nur auf Versuchsbasis in zwei Kliniken zur Behandlung von Personen unter 25 Jahren angewandt wurde. Der Patient konnte aufgrund seines Alters an diesen Versuchen nicht teilnehmen. Den Antrag auf Übernahme der Behandlungskosten lehnte seine Krankenkasse ab, da eine angemessene Behandlung in den Niederlanden bei einem Leistungserbringer hätte erfolgen können, mit dem der zuständige Träger einen Vertrag geschlossen hatte. Der EuGH hatte in diesen beiden Fällen nun die Frage zu beantworten, ob eine nationale Regelung, die für eine Krankenhausbehandlung im Ausland eine vorherige Genehmigung verlangt, die Dienstleistungsfreiheit der Patienten unzulässig einschränkt. Zudem fragte das vorlegende niederländische Gericht, ob es für die Beantwortung dieser Frage von Bedeutung sei, dass die betreffende Behandlung nicht als in ärztlichen Kreisen üblich betrachtet werde und darum nicht im inländischen Leistungskatalog enthalten sei.

Der EuGH hatte zunächst zu prüfen, ob die betreffenden Regelungen des EGV zur Dienstleistungsfreiheit überhaupt auf Krankenhausleistungen anzuwenden sind, da sie - wie einige Regierungen der Mitgliedstaaten argumentierten - insbesondere dann keine Dienstleistungen im Sinne von Art. 50 EGV $^{118}$ darstellen könnten, wenn sie im Rahmen des betreffenden Krankenversicherungssystems kostenlos erbracht würden. ${ }^{119}$ Der Gerichtshof entschied, dass auch medizinische Tätigkeiten von Art. 50 des Vertrags erfasst sind und damit Dienstleistungen darstellen, unabhängig davon, ob die $\mathrm{Be}-$ handlung in einer Krankenanstalt oder außerhalb erbracht wird. ${ }^{120} \mathrm{Da}$ auch Leistungen einer Krankenhausbehandlung gegen Geld erbracht würden, würden für sie grundsätzlich auch die Grundfreiheiten gelten, und zwar auch dann, wenn sie der Patient nicht direkt bezahle, sondern eine (pauschale) Vergütung durch eine Krankenkasse erfolge, denn auch dann stelle diese Vergütung eine Honorierung für eine wirtschaftliche Tä-

\footnotetext{
${ }^{114} \mathrm{Vgl}$. Europäischer Gerichtshof (2001a).

${ }^{115} \mathrm{Vgl}$. Europäischer Gerichtshof (2001b).

${ }^{116} \mathrm{Vgl}$. Europäischer Gerichtshof (2003e).

$117 \mathrm{Vgl}$. Europäischer Gerichtshof (2006).

${ }^{118}$ Nach Art. 50 EGV gehört zu den Kennzeichen von Dienstleistungen, dass sie gegen Entgelt erbracht werden.

${ }^{119} \mathrm{Vgl}$. Europäischer Gerichtshof (2001a), Rn. 48.

${ }^{120}$ Der EuGH entschied in seiner Urteilsbegründung nicht zwischen ambulanten und stationären Leistungen, sondern zwischen Krankenhausleistungen und Leistungen außerhalb eines Krankenhauses.
} 
tigkeit dar. ${ }^{121}$ Eine Regelung, die die Kostenübernahme für eine Auslandsbehandlung an eine vorherige Genehmigung knüpfe, beeinträchtige damit die Dienstleistungsfreiheit. ${ }^{122}$ Der Gerichtshof sah jedoch in diesem Fall eine Einschränkung der Grundfreiheiten durch zwingende Gründe des Allgemeininteresses begründet. Ein solcher Grund sei im Fall von Krankenhausbehandlungen darin zu sehen, dass Planungsanstrengungen nötig seien, um ein rationelles, stabiles, ausgewogenes und gut zugängliches Angebot an Krankenhauspflege zu gewährleisten. Eine solche Planung ist nach Ansicht des Gerichts nicht möglich, wenn es den Versicherten unter allen Umständen freistünde, Krankenanstalten im In- oder Ausland aufzusuchen, mit denen ihr Träger keine Verträge abgeschlossen habe.

Der Gerichtshof erkennt damit an, dass ein Genehmigungserfordernis im Falle von Krankenhausleistungen nach europäischem Recht zu rechtfertigen ist, obwohl es die Dienstleistungsfreiheit einschränkt. Darüber hinaus prüfte er die in den Niederlanden bestehenden Voraussetzungen für die Erteilung einer solchen Genehmigung. Demnach durfte eine Genehmigung nur erteilt werden, wenn eine ausreichende und angemessene Behandlung im Inland nicht möglich und die gewünschte Behandlung in ärztlichen Kreisen als üblich anerkannt war, da sie nur dann zum niederländischen Leistungskatalog zählte. Zur ersten Bedingung stellte der EuGH klar, dass nationalen Einrichtungen, mit denen der Träger keinen Vertrag abgeschlossen hat, kein Vorrang gegenüber Einrichtungen in anderen Mitgliedstaaten eingeräumt werden darf, wenn eine entsprechende Behandlung in inländischen Vertragseinrichtungen nicht möglich ist. In Bezug auf die zweite Genehmigungsvoraussetzung entschied der Gerichtshof, dass die Ablehnung der Kostenübernahme für Auslandsbehandlungen mit der Begründung, sie sei in ärztlichen Kreisen nicht als üblich angesehen, nur dann zu rechtfertigen ist, wenn sich die Üblichkeit einer Behandlung auf eine hinreichende Erprobung und Anerkennung in der internationalen Medizin bezieht. Bestimmt allein die wissenschaftliche Meinung inländischer Mediziner die Üblichkeit, so sei eine Bevorzugung der inländischen Leistungserbringer nicht auszuschließen und würde die Dienstleistungsfreiheit beschränken. ${ }^{123}$

Ebenfalls am 12.7.2001 verkündete der Europäische Gerichtshof das Urteil in der Rechtssache „Vanbraekel“. In diesem Fall hatte sich die Frau des Klägers, eine belgische Staatsangehörige, zu einer stationären Behandlung nach Frankreich begeben, obwohl der zuständige Träger zuvor den Antrag auf eine Genehmigung abgelehnt hatte, da ein nach belgischem Recht vorgeschriebenes Gutachten nicht erbracht worden war. Dieses Gutachten sollte dem Nachweis dienen, dass eine vorzunehmende Behandlung im Ausland unter besseren medizinischen Bedingungen durchzuführen sei, als es in Belgien möglich wäre. $\mathrm{Da}$ ein nationales Gericht bereits entschieden hatte, dass der

\footnotetext{
${ }^{121}$ Vgl. Europäischer Gerichtshof (2001a), Rn. 54-59.

${ }^{122} \mathrm{Vgl}$. Europäischer Gerichtshof (2001a), Rn. 69.

${ }^{123}$ Es ist zu beachten, dass dies nicht bedeutet, dass die Mitgliedstaaten nun verpflichtet sind, ihre Leistungskataloge an einen ,internationalen Standard“ anzupassen. Die Erforderlichkeit und Üblichkeit bezieht sich lediglich auf die Durchführung einer im nationalen Leistungskatalog vorgesehen Leistung. Die Definition dieses Leistungskataloges selbst bleibt aber in der alleinigen Kompetenz der Mitgliedstaaten. Vgl. Bieback (2001), S. 568.
} 
Träger die Genehmigung zu unrecht abgelehnt hatte und er darum die Kosten für die Behandlung übernehmen musste, blieb für den EuGH lediglich zu klären, in welcher Höhe die Kosten zu erstatten waren. Nach Artikel 22 Abs. 1 lit. c) der Verordnung 1408/71/EWG hat ein Sozialversicherter, dem eine Genehmigung zu einer Auslandsbehandlung erteilt wurde, Anspruch auf Sachleistungen vom zuständigen Träger des ausländischen Mitgliedstaats auf Basis der dort geltenden Rechtsvorschriften, als ob er dort versicherte wäre. Die Kosten der Leistungen trägt seine heimische Krankenkasse. Im hier betrachteten Fall waren die Behandlungskosten im Ausland geringer gewesen, als sie es bei einer entsprechenden Behandlung im Inland gewesen wären. Allerdings hatte die Patientin deutlich höhere Zuzahlungen als im Inland zu leisten. Der EuGH entschied, dass der inländische Kostenträger in diesem Fall eine zusätzliche Erstattung zu leisten habe, soweit der insgesamt geleistete Betrag die inländischen Behandlungskosten nicht übersteigt. Der EuGH begründete seine Entscheidung damit, dass es einen Versicherten davon abschrecken könne, Leistungserbringer im Ausland aufzusuchen und somit eine Behinderung des freien Dienstleistungsverkehrs darstelle, wenn dieser Versicherte im Fall einer Behandlung in einem anderen Mitgliedstaat eine geringere Erstattung erhalte, als er bei der gleichen Behandlung in seinem Heimatstaat beanspruchen könne.

Es bestand nach diesem Urteil jedoch keine endgültige Klarheit hinsichtlich der Frage, wann eine zusätzliche Erstattung nun tatsächlich zu gewähren ist. Zunächst ist dabei festzustellen, dass Art. 34 Abs. 5 der Verordnung 574/72 eine die tatsächlichen Kosten übersteigende Erstattung verbietet. ${ }^{124}$ Das EuGH-Urteil konnte also nicht bedeuten, dass eine höhere Erstattung dann erfolgen muss, wenn die gesamten Behandlungskosten niedriger ausfallen als im Inland. Im vorliegenden Fall waren die nach französischem Recht erstattungsfähigen Kosten zwar geringer als die Kosten, die bei einer entsprechenden Behandlung in Belgien von der dortigen Krankenversicherung übernommen werden, allerdings fallen in Frankreich erheblich höhere Zuzahlungen an. Von einigen Autoren wird darum die Ansicht vertreten, ${ }^{125}$ der EuGH wollte in seinem Urteil erreichen, dass Kostenvorteile durch niedrigere erstattungsfähige Kosten im Ausland nicht dem Träger zugute kommen, der die Genehmigung für die Behandlung zuvor zu Unrecht abgelehnt hat. Die Krankenkasse wird auf diese Weise gewissermaßen für eine unrechtmäßig verwehrte Genehmigung bestraft. Hätte sie die Genehmigung zur Auslandsbehandlung jedoch vorher erteilt, so hätte die Patientin nach dieser Auffassung die anfallenden Zuzahlungen selbst tragen müssen.

Das Urteil kann aber auch dahingehend interpretiert werden - und die EUKommission folgte dieser Auffassung in Art. 23 ihres Vorschlags für eine Richtlinie über Dienstleistungen im Binnenmarkt ${ }^{126}$-, dass ein Patient, der eine entsprechende Genehmigung erhalten hat, Anspruch auf eine zusätzliche Rückerstattung besitzt, wenn es z.B. aufgrund höherer Zuzahlungen im Ausland zu einer Deckungslücke kommt, und zwar bis zur Höhe der Differenz zwischen den im Ausland angefallenen

\footnotetext{
${ }^{124}$ Vgl. auch Kingreen (2001), S. 3383.

${ }^{125} \mathrm{Vgl}$. Kingreen (2001), S. 3383 und ähnlich Novak (2001), 476ff.

${ }^{126}$ Vgl. Europäische Kommission (2004c), Art. 23 Abs. 3.
} 
Kosten und dem höheren Betrag, der bei einer Behandlung im Inland entstanden wäre. ${ }^{127}$ Das Urteil selbst schafft hinsichtlich dieser Frage jedoch keine letztendliche Klarheit. ${ }^{128}$ Der EuGH ging auf diese Frage nochmals im Jahre 2006 im Urteil zur Rechtssache „Watts" ein, das weiter unten in diesem Abschnitt dargestellt ist.

Nachdem der EuGH nun in der Rechtssache „Smits und Peerbooms“ die Frage entschieden hatte, inwieweit ein Genehmigungsvorbehalt im Fall von Krankenhausleistungen mit der Dienstleistungsfreiheit vereinbar ist, war in der Rechtssache „MüllerFauré und van Riet " noch zu klären, wie diesbezüglich bei ambulanten Leistungen zu verfahren ist, die auf Basis des Sachleistungsprinzips erbracht werden. Zwar hatte der Gerichtshof bereits im "Smits/Peerbooms"-Urteil darauf hingewiesen, dass auch für die nach dem Sachleistungsprinzip organisierten Systeme die Grundfreiheiten des EGV grundsätzlich gelten, offen war jedoch weiterhin, ob für in diesen Systemen auBerhalb von Krankenhäusern erbrachte Leistungen ein Genehmigungsvorbehalt zu rechtfertigen ist, um - analog zur Begründung des EuGH in Bezug auf Krankenhausleistungen - die finanzielle Stabilität des Systems nicht zu gefährden. Im Urteil zur Rechtssache „Müller-Fauré und van Riet“ im Jahr 2003 verneinte dies der EuGH mit der Begründung, dass in diesem Bereich aufgrund von Sprachbarrieren, räumlicher Entfernung, Kosten eines Auslandsaufenthalts und fehlenden Informationen über die medizinische Versorgung im Ausland mit einer wirksamen Beschränkung der Behandlungszahlen gerechnet werden könne. ${ }^{129}$ Im Übrigen sei es den Krankenversicherungssystemen nach europäischem Recht möglich, die Erstattung für Auslandsbehandlungen auf diejenigen Kosten zu beschränken, die bei einer entsprechenden Behandlung im Inland entstanden wären. Die Mitgliedstaaten, die über ein Sachleistungssystem verfügen, dürfen in diesem Zusammenhang Erstattungsbeiträge für Auslandsbehandlungen festsetzen, wenn sie auf objektiven, nicht diskriminierenden und transparenten Kriterien beruhen.

Abgerundet wurde die Rechtsprechung zur grenzüberschreitenden Inanspruchnahme von Gesundheitsleistungen mit dem Urteil des EuGH in der Rechtssache ,Watts " vom 16. Mai 2006. ${ }^{130}$ Nachdem sich der Gerichtshof bisher mit Fragen zur Kostenübernahme von Auslandsbehandlungen innerhalb sozialer Krankenversicherungssysteme beschäftigt hatte, ging es in diesem Fall um die grenzüberschreitende Nachfrage einer Patientin des englischen National Health Service (NHS), dessen Finanzierung aus Steuermitteln erfolgt. Die Patientin, Yvonne Watts, litt an Hüftarthritis und beantragte beim Bedford Primary Care Trust (Bedford PCT) eine Genehmigung zur Behandlung im Ausland. Diese Genehmigung wurde nach einer Untersuchung im Oktober 2002 durch einen Facharzt, der sie als „Routinefall“ einstufte, abgelehnt. Damit hätte sich für sie bis zur Operation eine Wartezeit von einem Jahr ergeben. Der Bedfort PCT begründete seine Ablehnung damit, dass die Patientin ,innerhalb der Zielvorgaben der Regierung für den NHS“ und damit „rechtzeitig“ am Wohnort behandelt werden kön-

\footnotetext{
${ }^{127} \mathrm{Vgl}$. Jorens/Coucheir/Overmeiren (2005), S. 16f.

${ }^{128} \mathrm{Vgl}$. zu dieser Diskussion auch Fuchs (2005), S. 256 sowie die dort angegebene Literatur.

${ }^{129} \mathrm{Vgl}$. Europäischer Gerichtshof (2003e), Rn.

${ }^{130} \mathrm{Vgl}$. Europäischer Gerichtshof (2006).
} 
ne. ${ }^{131}$ Aufgrund einer Verschlechterung des Gesundheitszustands der Patientin erfolgte im Januar 2003 eine weitere Untersuchung. Daraufhin sollte die Operation innerhalb von zwei bis drei Monaten erfolgen. Eine Genehmigung zur Auslandsbehandlung lehnte der Bedford PCT erneut ab. Die Patientin ließ sich dennoch im März 2003 in Frankreich behandeln und beantragte eine Erstattung der entstandenen Kosten, die der Bedford PCT ebenfalls ablehnte. Im nachfolgenden Rechtsstreit legte das zuständige englische Gericht dem EuGH verschiedene Fragen zur Vorabentscheidung vor. Zum einen ging es um die Frage, ob die Ablehnung der Behandlung unzulässig war und die Patientin demnach einen Anspruch auf eine Erstattung der Kosten hat. Zum anderen fragte das Gericht, ob sich für Patienten des NHS direkt aus der Dienstleistungsfreiheit ein Anspruch auf eine grenzüberschreitende Nachfrage nach Gesundheitsleistungen ergibt.

Der EuGH entschied, dass die Verpflichtung zur Übernahme von Kosten für Krankenhausbehandlungen in einem anderen Mitgliedstaat grundsätzlich auch für den NHS gilt, obwohl dieser derartige Leistungen kostenfrei erbringt. Eine Genehmigung zur Auslandsbehandlung darf dem Patienten nur dann unter Hinweis auf das Bestehen einer Warteliste versagt werden, wenn nachzuweisen ist, dass die Wartezeit bis zur Behandlung im Wohnsitzland nicht den in Anbetracht des Gesundheitszustands und des klinischen Bedarfs des Betroffenen medizinisch vertretbaren zeitlichen Rahmen überschreitet.

Zudem führt der Gerichtshof aus, dass unabhängig von der Funktionsweise des nationalen Systems Fälle grenzüberschreitender Leistungsinanspruchnahme immer in den Anwendungsbereich der Bestimmungen über den freien Dienstleistungsverkehr fallen. Analog zu seinen Entscheidungen in den Rechtssachen "Smits und Peerbooms“ und „Müller-Fauré und van Riet" betonte der EuGH, dass ein Genehmigungsvorbehalt für Patienten und Leistungserbringer eine Beschränkung der Dienstleistungsfreiheit darstelle, eine solche Beschränkung im Fall von Krankenhausbehandlungen jedoch aufgrund zwingender Gründe zu rechtfertigen sei. Der Gerichtshof kritisierte aber, dass die den NHS betreffenden Regelungen nicht die Kriterien für die Erteilung einer Genehmigung festlegen und damit dem Ermessen der zuständigen nationalen Stelle keine Grenzen setzen. Dies erschwere auch die gerichtliche Kontrolle der Entscheidung.

In Bezug auf die - durch das „Vanbraekel“-Urteil nicht eindeutig beantwortete - Frage nach der Höhe der zu übernehmenden Kosten stellte der EuGH klar, dass Patienten bei einer Auslandsbehandlung einen Anspruch auf Erstattung besitzen, der dem der Versicherten des Landes entspricht, in dem die Behandlung erfolgt. Außerdem müsse der NHS auch mögliche Eigenbeteiligungen übernehmen, sofern der gesamte Erstattungsbetrag die Kosten einer vergleichbaren Behandlung im Inland nicht übersteigt. Die kostenfreie Erbringung der Leistungen in England stelle dabei keinen Hinderungsgrund dar, da ein fiktiver Preis auch im nationalen Gesundheitsdienst nach objektiven Kriterien ermittelt werden könne. Damit wird deutlich, dass es eine ungerechtfertigte Beschränkung des freien Dienstleistungsverkehrs darstellt, wenn ein Versicherter, der

${ }^{131}$ Vgl. Europäischer Gerichtshof (2006), Rn. 26. 
eine Genehmigung für eine Krankenhausbehandlung im Ausland erhalten hat, keine Kostenübernahme in gleicher Höhe beanspruchen kann wie sie ihm bei einer Behandlung im Inland zugestanden hätte. Demnach haben die Versicherten im Fall einer Genehmigung der Auslandsbehandlung Anspruch auf die Übernahme von im Ausland entstandenen Zuzahlungen, solange der insgesamt vom inländischen Finanzierungsträger erstattete Betrag die Kosten nicht übersteigt, die dem Träger durch eine entsprechende Behandlung im Inland entstanden wären. Die Kosten für Reise und Unterbringung müssen aber nur dann übernommen werden, wenn dies bei einer entsprechenden Behandlung im Inland vorgesehen ist.

Mit diesen Urteilen hat der EuGH nun den Rahmen für die Inanspruchnahme von Auslandsleistungen im ambulanten und stationären Sektor abgesteckt. Diese Rechtsprechung ist allerdings auch acht Jahre nach den Kohll/Decker-Urteilen noch immer nicht von allen Mitgliedstaaten in nationales Recht umgesetzt worden. Da zudem das Europäische Parlament im Februar 2006 die Regelungen zur grenzüberschreitenden Kostenübernahme bei Gesundheitsleistungen aus dem Kommissions-Vorschlag für eine Dienstleistungsrichtlinie herausstrich, existiert in diesem Bereich bisher keine EU-weit einheitliche Regelung. In Deutschland fand die Rechtsprechung mit dem Gesetz zur Modernisierung der Gesetzlichen Krankenversicherung (GKV-Modernisierungsgesetz, GMG) im Jahre 2004 Berücksichtigung in den $\S \S 13$ Abs. 4 und 5, 18 und 140e Sozialgesetzbuch (SGB) V. Seither ist eine Inanspruchnahme von Leistungen im EWR auf Basis der Kostenerstattung auch nach dem deutschen Sozialrecht möglich. Damit besteht im Fall von außerhalb von Krankenhäusern erbrachten Leistungen neben der Inanspruchnahme mit vorheriger Genehmigung der Krankenkasse eine zweite Möglichkeiten zur grenzüberschreitenden Behandlung, die sich direkt aus den Grundfreiheiten des EGV ableitet. ${ }^{132}$ Eine Genehmigung vor einer Auslandsbehandlung ist lediglich bei Krankenhausleistungen weiterhin zwingend erforderlich. Für diese Leistungen bleiben damit die Bestimmungen der Verordnung 1408/71/EWG die alleinige Möglichkeit zur Kostenübernahme. Durch die Entscheidung des EuGH in der Rechtssache „Inzian“ vom 23.10.2003 bleiben beide Verfahren - mit und ohne Genehmigung -, die die Kostenübernahme von in einem anderen Mitgliedstaat in Anspruch genommenen Leistungen regeln, nebeneinander bestehen, ohne dass eines einen Vorrang erhält. ${ }^{133} \mathrm{Im}$ entsprechenden Urteil stellte der Gerichtshof klar, dass die Regelungen der Verordnung 1408/71/EWG dazu beitrügen, die Freizügigkeit der Sozialversicherten zu erhöhen, da sie den Patienten Rechte einräumen, die sie andernfalls nicht hätten. Somit stelle die Möglichkeit, Leistungen im Ausland außerhalb von Krankenhäusern weiterhin mit vorheriger Genehmigung der Krankenkasse in Anspruch nehmen zu können, entgegen

\footnotetext{
${ }^{132}$ Die beiden Verfahren unterscheiden sich in der Art der Abrechnung der entstandenen Kosten sowie in der Höhe der vom zuständigen Kostenträger übernommenen Behandlungskosten (vgl. dazu Abschnitt 3.2.1). Welches Verfahren für den Versicherten vorteilhafter ist, lässt sich nur im Einzelfall entscheiden. Darum ist das Nebeneinander beider Verfahren für außerhalb von Krankenhăusern erbrachten Leistungen auch weiterhin sinnvoll.

${ }^{133} \mathrm{Vgl}$. Jorens/Coucheir/Overmeiren (2005), S. 12.
} 
der Argumentation der klagenden französischen Krankenkasse, keine Beschränkung der Dienstleistungsfreiheit dar. ${ }^{134}$

\subsubsection{Die aktive Dienstleistungsfreiheit für Angehörige von Gesund- heitsberufen}

In der oben dargestellten Rechtsprechung ging es vordergründig lediglich um die Frage, inwieweit die Forderung nach einer vorherigen Genehmigung für die Inanspruchnahme von Gesundheitsdienstleistungen im Ausland als Voraussetzung für eine Kostenübernahme der gesetzlichen Krankenkassen die passive Dienstleistungsfreiheit der Versicherten verletzt. Dieselbe Rechtsprechung kann jedoch auch zur Bewertung des Verhältnisses zwischen Leistungserbringern, die sich für eine vorübergehende Erbringung von Dienstleistungen ins Ausland begeben, und den nationalen Krankenversicherungssystemen herangezogen werden. Einen dementsprechenden Hinweis enthält auch das Urteil des EuGH in der Rechtssache „Smits und Peerbooms“, in dem der Gerichtshof betont, dass die dort betrachtete Regelung einer vorherigen Genehmigung als Voraussetzung für die Kostenübernahme bei Auslandleistungen sowohl für die Versicherten als auch für die Leistungserbringer eine Behinderung des freien Dienstleistungsverkehrs darstelle. ${ }^{135}$ Jorens et al. (2005) unternehmen auf dieser Basis einen Versuch, den europäischen Rechtsrahmen abzustecken, in dem ausländische Angehörige von Gesundheitsberufen tätig werden, wenn sie in einem anderen Mitgliedstaat vorübergehend Dienstleistungen erbringen. ${ }^{136}$ Nach Meinung der Autoren besteht grundsätzlich ein Anspruch auf Kostenübernahme durch das inländische Sicherungssystem, wenn Patienten ausländische Leistungserbringer aufsuchen, unabhängig davon, ob sich die Patienten zur Inanspruchnahme ins Ausland begeben oder ob die Leistungserbringer vorübergehend im Mitgliedstaat der Versicherungszugehörigkeit des Patienten tätig werden. Dabei könne von den ausländischen Leistungserbringern nicht erwartet werden, dass sie mit allen Krankenversicherungsträgern, bei denen ihre potenziellen Patienten versichert sein könnten, Verträge abschließen. Nach dieser Interpretation der EuGH-Urteile würden für die vorübergehende Erbringung von Gesundheitsdienstleistungen folgende Bedingungen gelten: ${ }^{137}$

- Wenn der Dienstleistungserbringer Leistungen anbietet, deren Kosten die inländische Krankenkasse bei einer Erbringung durch einen im Inland niedergelassenen vertraglichen Dienstleister übernommen hätte, so hat der Patient Anspruch auf eine Erstattung, deren Höhe nicht unter der liegen darf, die gewährt wird, wenn eine entsprechende Leistung von einem inländischen vertraglichen Dienstleister erbracht wird.

- Der Patient muss keine vorherige Genehmigung einholen, wenn es sich um Leistungen handelt, die außerhalb von Krankenhäusern erbracht werden. Davon unbe-

\footnotetext{
${ }^{134} \mathrm{Vgl}$. Europäischer Gerichtshof (2003f), Rn. 21-22 sowie 51-60.

${ }^{135} \mathrm{Vgl}$. Europäischer Gerichtshof (2001a), Rn. 69.

${ }^{136} \mathrm{Vgl}$. Jorens/Coucheir/Overmeiren (2005), S. 25ff.

${ }^{137} \mathrm{Vgl}$. Jorens/Coucheir/Overmeiren (2005), S. $30 \mathrm{f}$.
} 
rührt bleiben Genehmigungen, die in den inländischen Regelungen zur Kostenübernahme generell für die Inanspruchnahme einer Leistung vorgesehen sind. ${ }^{138}$

- Der Leistungserbringer sollte die gleichberechtigte Möglichkeit erhalten, an dem im Aufnahmemitgliedstaat anwendbaren Vertragssystem teilzunehmen, wobei die Prüfung seiner Bewerbung auf objektiven, transparenten und nicht-diskriminierenden Kriterien beruhen muss.

- Die Mitgliedstaaten behalten die volle Kompetenz bezüglich der Festlegung des persönlichen Geltungsbereichs ihres Systems, der abgedeckten Behandlungen und dem Ausmaß ihrer Abdeckung sowie der Bedingungen, unter denen Leistungen gewährt werden, wenn diese Regelungen unterschiedslos auf in- und ausländische Leistungserbringer angewandt werden, auch wenn sie die Erbringung durch ausländischen Leistungsanbieter erschweren oder verhindern. ${ }^{139}$

Nach dieser Interpretation von Jorens et al. würde die Rechtsprechung des EuGH bezüglich der Geltung der Dienstleistungsfreiheit im Gesundheitswesen zu einer erleichterten Kostenübernahme im Fall einer Inanspruchnahme von Gesundheitsleistungen bei ausländischen Anbietern führen, die lediglich vorübergehend im Inland tätig sind. Es bliebe aber dabei, dass ausländische Ärzte bei der Erbringung von Dienstleistungen auch die gleichen Pflichten besäßen wie die Staatsangehörigen des Aufnahmestaats; insbesondere unterlägen sie den beruflichen und administrativen Disziplinarvorschriften dieses Mitgliedstaats. ${ }^{140}$

Im Bereich der aktiven Dienstleistungsfreiheit hätte es bedeutende Veränderungen gegeben, wenn der Vorschlag der EU-Kommission für eine Richtlinie über Dienstleistungen im Binnenmarkt ${ }^{141}$ in der ursprünglichen Fassung umgesetzt worden wäre. Kernpunkt dieses Vorschlags war die Anwendung des Herkunftslandprinzips auf die Dienstleistungserbringung und somit auch auf die Erbringung von Gesundheitsleistungen. Demnach hätte ein ausländischer Leistungserbringer, wenn er nur vorübergehend in Deutschland tätig gewesen wäre, lediglich die Bestimmungen seines Heimatlandes zu beachten gehabt, um eine Dienstleistung erbringen zu dürfen. Dies bezog sich insbesondere auf die Qualität und den Inhalt der Dienstleistung, die Verträge, Haftungsfragen sowie sonstige nationale Bestimmungen des Herkunftsmitgliedstaates, die die Aufnahme und Ausübung der Dienstleistungen regeln. ${ }^{142}$ Für die Kontrolle des Leistungserbringers wäre die zuständige Behörde des Herkunftslandes verantwortlich geblieben.

In der im Februar 2006 vom Europäischen Parlament in erster Lesung verabschiedeten Fassung der Dienstleistungsrichtlinie sind jedoch - neben anderen Bereichen - auch

${ }^{138}$ So ist beispielsweise in Deutschland bei Zahnersatzleistungen die vorherige Genehmigung eines Heil- und Kostenplans Voraussetzung für die Kostenübernahme der deutschen Krankenkasse.

${ }^{139}$ Diese Bedingung muss gelten, um die Kompetenzen der Mitgliedstaaten fur die Organisation des Gesundheitswesens und die medizinische Versorgung nicht zu verletzten, die ihnen laut Art. 152 Abs. 5 EGV zukommen. Vgl. Jorens/Coucheir/Overmeiren (2005), S. 31.

${ }_{140}$ Vgl. Art. 17 Abs. 1 Satz 2 Richtlinie 93/16/EWG.

141 Vgl. Europäische Kommission (2004c).

${ }^{142}$ Vgl. o.V. (2005d), S. 25 
die Gesundheitsdienstleistungen nicht mehr enthalten, so dass die Richtlinie keine Relevanz mehr für das Gesundheitswesen besitzt. ${ }^{143}$ Der Gesundheitsbereich soll von einer eigenen Richtlinie erfasst werden. Die Kommission hat angekündigt, einen entsprechenden Vorschlag im Jahre 2007 vorzulegen, der u.a. Fragen der Kostenerstattung furr Behandlungen im europäischen Ausland und der Haftung bei entstandenen Schäden behandeln soll. ${ }^{144}$ Der Generalanwalt am EuGH Alber forderte nach dieser Ankündigung der Kommission, auch die Rechte der Leistungserbringer bei der grenzüberschreitenden Ausübung ihres Berufes zu berücksichtigen, die sich aus der aktiven Dienstleistungsfreiheit ergeben. ${ }^{145}$ Neben den Fragen zur Kostenerstattung sollte nach Ansicht von Alber auch die Anbieterseite berücksichtigt werden, indem bürokratische Hürden beseitigt würden, mit denen nationale Behören versuchten, die Berufsausübung ausländischer Ärzte, Zahnärzte oder Pflegekräfte zu behindern. Da gegen eine Richtlinie für Gesundheitsdienstleistungen jedoch Widerstände aus verschiedenen EUStaaten, z.B. Frankreich und Großbritannien bestehen, bleibt abzuwarten, ob die Initiative der Kommission auf kurze Sicht Erfolg haben wird und zu einer schrittweisen Öffnung der nationalen Märkte beitragen kann.

\subsection{Zur Geltung des europäischen Wettbewerbsrechts}

Neben den vier Grundfreiheiten bildet das europäische Wettbewerbsrecht ein weiteres Kernstück zur Verwirklichung eines unionsweiten Binnenmarkts. Die entsprechenden Regelungen dienen dem Schutz des Wettbewerbs vor einvernehmlichen Beschränkungen durch einzelne Marktteilnehmer (Art. 81 EGV) sowie vor der Übermacht einzelner Marktteilnehmer gegenüber anderen Marktteilnehmern (Art. 82 EGV). Art. 86 EGV bestimmt, dass auch öffentliche Unternehmen grundsätzlich dem Wettbewerbsrecht unterliegen, so dass allein aus der Tatsache, dass die deutschen Krankenkassen öffentlich-rechtliche Körperschaften sind, nicht zwangsläufig geschlossen werden kann, dass auf sie die Bestimmungen des Wettbewerbsrechts nicht anzuwenden sind. ${ }^{146}$ Unternehmen, die eine Dienstleistung von allgemeinem wirtschaftlichem Interesse ${ }^{147}$ erbringen, sind nur dann von diesen Regeln befreit, wenn deren Anwendung die Erfüllung der ihnen übertragenen besonderen Aufgaben verhindert. In Bezug auf die Sozialversi

\footnotetext{
${ }^{143} \mathrm{Vgl}$. Spielberg (2006), S. 454.

${ }^{144} \mathrm{Vgl}$. Europäische Kommission (2006b).

${ }^{145} \mathrm{Vgl.} \mathrm{o.V.} \mathrm{(2006),} \mathrm{S.} \mathrm{A} 1780$.

${ }^{146} \mathrm{Vgl}$. Eichenhofer (2001), S. 1.

${ }^{147}$ Dieser Begriff wird in Art. 16 und Art. 86 Abs. 2 EGV verwendet, ist aber weder im Vertrag selbst noch im abgeleiteten Recht näher bestimmt. Es herrscht jedoch weitgehende Übereinstimmung, dass er sich auf wirtschaftliche Tätigkeiten bezieht, die von den Mitgliedstaaten oder der Gemeinschaft mit besonderen Gemeinwohlverpflichtungen verbunden und im Interesse der Allgemeinheit erbracht werden. Dies beinhaltet u.a. Leistungen des Verkehrswesens, der Postdienste, des Energiesektors und der Telekommunikation, aber auch jede sonstige wirtschaftliche Tătigkeit in Zusammenhang mit Gemeinwohlverpflichtungen, z.B. Abfallwirtschaft und sozialer Wohnungsbau. Vgl. o.V. (2005a), S. 4, Fn. 2.
} 
cherungsträger stellen sich in dieser Hinsicht drei, jeweils aufeinander aufbauende Fragen: ${ }^{148}$

- Sind sie Unternehmen nach europäischem Recht?

- Sind sie als Unternehmen mit Dienstleistungen von allgemeinem wirtschaftlichem Interesse betraut?

- Welche Wettbewerbsregeln könnten in diesem Fall die rechtliche und tatsächliche Erfüllung der ihnen übertragenen besonderen Aufgaben verhindern?

Der $\mathrm{EuGH}^{149}$ geht von einem funktionalen, also tätigkeitsbezogenen Unternehmensbegriff aus, d.h. sein Unternehmensbegriff erfasst, unabhängig von der Rechtsform oder der Art der Finanzierung, jede Wirtschaftseinheit, die eine wirtschaftliche Tätigkeit ausübt. ${ }^{150}$ Der Begriff bezieht sich damit auf die im konkreten Fall ausgeübte Tätigkeit, so dass ein und derselbe Rechtsträger für eine bestimmte Tätigkeit als Unternehmen eingestuft werden kann, für eine andere dagegen nicht.

Im Rahmen seiner Rechtsprechung hat der EuGH speziell in Bezug auf die Träger der Kranken- und Rentenversicherung Kriterien für die Beurteilung ihrer Tätigkeiten entwickelt. Danach üben diese Sozialversicherungsträger keine wirtschaftliche Tätigkeit aus, wenn sie mit ihrer Tätigkeit einen rein sozialen Zweck verfolgen und ihre Leistungen von Gesetzes wegen unabhängig von der Höhe der Beiträge erbringen. Nach diesen Kriterien stufte der Gerichtshof in der grundlegenden Entscheidung „Poucet und Pistre" den Träger eines französischen Pflichtversicherungssystems zur Absicherung der Risiken bei Krankheit und Mutterschaft nicht als Unternehmen ein, da das System auf dem Umlageverfahren basierte und bei der Leistungserbringung strikt an gesetzliche Vorgaben gebunden war. ${ }^{\text {si }}$ Im Urteil in der Rechtssache „Cisal“ begründete der EuGH die fehlende Unternehmenseigenschaft einer italienischen staatlichen Unfallversicherungsanstalt insbesondere damit, dass die Bemessung der Beitrags- und Leistungshöhe auf dem Grundsatz der Solidarität beruhte und die Höhe der Leistungen und Beiträge staatlich festgesetzt war. ${ }^{152}$ Dagegen stuft er einen Rentenfonds als Unternehmen ein, der zwar ohne Gewinnerzielungsabsicht soziale Ziele verfolgt und dessen Mitgliedschaft teilweise gesetzlich vorgeschrieben ist, der aber seine Beiträge selbst bestimmt und nach dem Kapitalisierungsprinzip arbeitet, also die Beiträge seiner Mitglieder investiert und die Gewinne verwendet, um damit die an seine Mitglieder auszuzahlenden Pensionen zu finanzieren. ${ }^{153}$

Bei den genannten Entscheidungen ging es stets um die Bewertung der Beziehungen zwischen den Versicherungen und ihren Mitgliedern sowie die Beurteilung der von den Versicherungsträgern angebotenen Dienstleistungen. Da aber Krankenkassen als Nachfrager nach Gesundheitsgütern erheblichen Einfluss auf die jeweiligen Märkte

\footnotetext{
${ }^{148} \mathrm{Vgl}$. Eichenhofer (2001), S. 2.

${ }_{149} \mathrm{Vgl}$. z.B. Europäischer Gerichtshof (1991), Rn. 21; Europäischer Gerichtshof (1993b), Rn. 17.

${ }^{150} \mathrm{Vgl}$. im Folgenden Koenig/Engelmann (2004), S. 683.

${ }^{151} \mathrm{Vgl}$. Europäischer Gerichtshof (1993b), Rn. 7ff.

$152 \mathrm{Vgl}$. Europäischer Gerichtshof (2002), Rn. 38ff und $43 \mathrm{ff}$.

${ }^{153} \mathrm{Vgl}$. Europäischer Gerichtshof (1999), Rn. 79ff.
} 
ausüben können, stellt sich die Frage, unter welchen Voraussetzungen Krankenkassen sich in dieser Hinsicht an den Vorgaben der Bestimmungen des europäischen Wettbewerbs messen lassen müssen.

Konkret war dabei zu klären, ob allein das Anbieten von Gütern auf Märkten als wirtschaftliche Tätigkeit zu werten ist oder ob davon losgelöst auch die Güternachfrage eine wirtschaftliche Tätigkeit darstellt und somit Unternehmenseigenschaften begründet. In der Rechtssache „Fenin“ entschied das Gericht erster Instanz des Europäischen Gerichtshofes (EuG) im Jahre 2000, dass der relevante Begriff der wirtschaftlichen Tätigkeit gekennzeichnet ist durch das Anbieten von Gütern und Dienstleistungen auf einem bestimmten Markt und nicht durch die Einkaufstätigkeit als solche. ${ }^{154}$ Demnach ist die Beurteilung des Kaufs von Erzeugnissen nicht von der späteren Verwendung zu trennen, so dass der wirtschaftliche oder nicht wirtschaftliche Charakter der späteren Verwendung maßgeblich ist für den Charakter der Einkaufstätigkeit. ${ }^{155}$ Dies gilt auch dann, wenn die betreffende Einrichtung als Nachfrager eine erhebliche Marktmacht ausüben kann. ${ }^{156}$ Folglich ist für die Unternehmenseigenschaften von Sozialversicherungsträgern alleine der Charakter der angebotenen Versicherungsleistungen für die Beurteilung maßgeblich. Wenn Krankenversicherungen nach den in der „Poucet und Pistre"-Rechtsprechung genannten Kriterien nicht als Unternehmen einzustufen sind, handeln sie demnach auch dann nicht als Unternehmen, wenn sie die Leistungen zur Versorgung ihrer Mitglieder auf Märkten einkaufen. ${ }^{157}$ Somit gilt: „Die im Rahmen der Nachfragetätigkeit vorhandenen Steuerungsmöglichkeiten der Krankenkassen bleiben demnach außerhalb der Reichweite der EG-Kartellregeln."

In Bezug auf die deutschen gesetzlichen Krankenkassen hatte der EuGH in den verbundenen Rechtssachen C-264/01, C-306/01, C-354/01 und C-355/01 ${ }^{159}$ darüber zu entscheiden, inwieweit sie wirtschaftliche Tätigkeiten ausüben. Insbesondere ging es um die Frage, ob Krankenkassen bzw. ihre Verbände mit der einheitlichen und gemeinsamen Bestimmung von Festbeträgen gegen die Wettbewerbsvorschriften des EGV verstoßen. ${ }^{160}$ Der EuGH ging in seiner Urteilsbegründung nicht auf das „Fenin“Urteil ein, obwohl über die Unternehmenseigenschaften der Kassen als (indirekte) Nachfrager von Gütern zu entscheiden war, sein Urteil wies jedoch in die gleiche Richtung. Er prüfte zunächst, ob die Krankenkassen mit ihrer Haupttätigkeit, also der Erbringung der gesetzlich vorgeschriebenen Leistungen, eine wirtschaftliche Tätigkeit ausüben. $\mathrm{Zu}$ dieser Überprüfung zog der Gerichtshof die in der „Poucet und Pistre“Rechtsprechung entwickelten Kriterien heran und kam zu dem Schluss, dass die Krankenkassen in Deutschland bei der Mitarbeit an der Verwaltung des Systems der sozialen Sicherheit eine rein soziale Aufgabe wahrnähmen, die auf den Grundsatz der Soli-

\footnotetext{
${ }^{154} \mathrm{Vgl}$. Europäisches Gericht erster Instanz (EuG) (2003), Rn. 36 unter Hinweis auf Europäischer Gerichtshof (1998a), Rn. 36.

${ }^{155}$ Das Gericht folgt damit der Argumentation der Kommission.

${ }^{156} \mathrm{Vgl}$. Europäisches Gericht erster Instanz (EuG) (2003), Rn. 37.

${ }^{157} \mathrm{Vgl}$. Koenig/Engelmann (2004), S. 684.

${ }^{158}$ Koenig/Engelmann (2004), S. 684.

$159 \mathrm{Vgl}$. Europäischer Gerichtshof (2004b).

${ }^{160} \mathrm{Vgl}$. zur Kommentierung dieses Urteils Koenig/Engelmann (2004).
} 
darität beruhe und ohne Gewinnerzielungsabsicht ausgeübt werde. ${ }^{161}$ Zudem sei hervorzuheben, dass die Krankenkassen gesetzlich verpflichtet seien, ihren Mitgliedern im Wesentlichen gleiche Pflichtleistungen anzubieten, die unabhängig von der Beitragshöhe sind, und die Krankenkassen somit keine Möglichkeit besäßen, auf diese Leistungen Einfluss zu nehmen. Bei der Ausübung ihrer Hauptaufgabe seien sie demnach nach europäischem Recht nicht als Unternehmen einzustufen.

Dennoch ist es nach der EuGH-Rechtsprechung denkbar, dass Krankenkassen und ihre Verbände bei der Ausübung einzelner Aufgaben, die außerhalb ihrer Haupttätigkeit liegen, wirtschaftlich tätig sind. Darum prüfte der EuGH gesondert, ob die Kassenverbände speziell mit der Festsetzung von Festbeträgen für Arzneimittel eine wirtschaftliche Tätigkeit ausüben. Festbeträge geben die Obergrenze des Erstattungsbetrags für bestimmte Arzneimittel an. Übersteigt der Preis des Arzneimittels den jeweiligen Festbetrag, muss der Versicherte die Mehrkosten selbst tragen. § 35 SGB V sieht vor, dass der Gemeinsame Bundesausschuss von Ärzten und Krankenkassen zunächst Gruppen von Arzneimitteln festlegt, für die Festbeträge vereinbart werden. Diese Gruppen bestehen aus Arzneimitteln

- mit denselben Wirkstoffen,

- mit pharmakologisch-therapeutisch vergleichbaren Wirkstoffen, insbesondere mit chemisch verwandten Stoffen oder

- mit therapeutisch vergleichbarer Wirkung, insbesondere Arzneimittelkombinationen.

In einem zweiten Schritt bestimmen die Spitzenverbände der Krankenkassen die jeweiligen Festbeträge der definierten Gruppen gemeinsam und einheitlich. Es handelt sich dabei um Erstattungshöchstbeträge und nicht um eine zentrale Preisfestsetzung. Die Arzneimittelhersteller besitzen theoretisch die Möglichkeit, einen Preis oberhalb des Festbetrags zu setzen. Allerdings hat sich seit der Einführung der Festbetragsregelung mit dem Gesetz zur Strukturreform im Gesundheitswesen (GesundheitsreformGesetz, GRG) im Jahre 1989 gezeigt, dass die Hersteller sich ökonomisch gezwungen sahen, nahezu alle Preise der erfassten Arzneimittel auf Festbetragsniveau abzusenken bzw. festzulegen.

Nach Ansicht des EuGH erfüllen die Verbände damit eine gesetzliche Pflicht, die ihnen durch das SGB V innerhalb enger Vorgaben auferlegt wird; lediglich die Höhe der Festbeträge ist nicht durch das Gesetz bestimmt. Der Spielraum, den die Kassen hinsichtlich der konkreten Höhe besitzen, berühre jedoch einen Bereich, in dem sie nicht miteinander konkurrierten, da die Arzneimittelkosten von allen Kassen zu gleichen Bedingungen zu tragen seien. Darum handelten die Kassen bei der Festbetragssetzung nicht in einem wirtschaftlichen Interesse, das von ihrem rein sozialen Zweck zu trennen sei. Aus dieser Argumentation folgt, dass auch die Krankenkassenverbände bei der Festsetzung von Arzneimittelfestbeträgen nicht als Unternehmen anzusehen sind. Der EuGH bestätigt damit den Ansatz des EuG in der Rechtssache „Fenin“, da er allein auf

${ }^{161}$ Vgl. Europäischer Gerichtshof (2004b), Rn. 51 ff. 
den Charakter der von den Krankenkassen angebotenen Leistungen abstellt. Mit dieser Entscheidung des Gerichtshofs wurde eine Beantwortung der weiteren Fragen (vgl. S. 34) hinfällig, da die Wettbewerbsregeln des EGV und deren Ausnahmen nur für Unternehmen gelten.

Aus diesem EuGH-Urteil kann nun jedoch nicht darauf geschlossen werden, dass die gesetzlichen Krankenkassen in Deutschland heute keinerlei wirtschaftliche Tătigkeiten ausüben. Als Gegenbeispiel nennen Koenig und Engelmann das Angebot von integrierten Versorgungsformen kombiniert mit Bonusmodellen. Den Krankenkassen wurde hier im Vergleich zu den zuvor möglichen Versorgungsformen größere Spielräume zur individuellen Gestaltung ihrer Leistungen eingeräumt. ${ }^{162}$ Sollte sich außerdem in $\mathrm{Zu}-$ kunft das Krankenversicherungssystem dahingehend verändern, dass es zu einer weiteren Stärkung der Wettbewerbselemente und einer Vergrößerung der Spielräume der einzelnen Kassen hinsichtlich ihres Leistungsangebots kommt, könnte der EuGH bei einer erneuten Beurteilung sogar die Haupttätigkeit der Kassen als wirtschaftlich und diese damit als Unternehmen einstufen. ${ }^{163}$

Doch auch dann wären die Wettbewerbsgrundsätze der Art. 81 und 82 EGV nicht notwendigerweise in vollem Umfang auf die gesetzlichen Krankenversicherungen anwendbar. Wie bereits erwähnt, gelten diese Regelungen nur eingeschränkt für Unternehmen, die mit Dienstleistungen von allgemeinem wirtschaftlichem Interesse betraut $\operatorname{sind}^{164}$ und zwar soweit „die Anwendung dieser Vorschriften nicht die Erfüllung der ihnen übertragenen besonderen Aufgaben rechtlich oder tatsächlich verhindert." "165 Dieser Ausnahmetatbestand ist nach der Rechtsprechung des EuGH bereits dann erfüllt, wenn die Anwendung der Wettbewerbsvorschriften ,die Erfüllung der besonderen Verpflichtungen, die dem Unternehmen obliegen, sachlich oder rechtlich gefährden würde" - eine Bedrohung für das Überleben des Unternehmens muss dagegen nicht bestehen. ${ }^{160}$ Zudem muss die EU-Kommission und nicht der betroffene Mitgliedstaat nachweisen, dass die Erfüllung der Aufgaben nicht durch andere Maßnahmen sicherzustellen ist. Der Mitgliedstaat muss lediglich den Nachweis führen, dass die Anwendung der Vorschriften die Erfüllung der dem Unternehmen übertragenen Aufgaben behindert. ${ }^{167}$ Dennoch bestünde dann keine generelle Ausnahme von den Wettbewerbsvorschriften für die Sozialversicherungsträger mehr, vielmehr wäre in jedem konkreten Einzelfall eine Verhältnismäßigkeitsprüfung notwendig. ${ }^{168}$

\footnotetext{
${ }^{162} \mathrm{Vgl}$. Koenig/Engelmann (2004), S. 686. 87). Ähnlich auch Hänlein/Kruse (2000), S. 172.

${ }^{165}$ Art. 86 Abs. 2 EGV.

${ }^{166}$ Vgl. Europäischer Gerichtshof (1997), Rn. 59.

${ }^{167} \mathrm{Vgl}$. Europäischer Gerichtshof (1997), Rn. $101 \mathrm{ff}$.

${ }^{168}$ Vgl. Riedel (2004), S. 245.
}

${ }^{163}$ Vgl. auch Riedel (2004), S. 245; Neumann-Duesberg (1998), S. 27.

${ }^{164}$ Der Generalanwalt des EuGH betonte in seinen Schlussanträgen vom 22.05.2003 zu den verbundenen Rechtssachen „AOK Bundesverband u.a.“, dass die gesetzlichen Krankenkassen in Deutschland mit solchen Dienstleistungen betraut sind, nämlich mit der Bereitstellung eines auf Solidarităt beruhenden Systems der gesetzlichen Krankenversicherung. Vgl. Europäischer Gerichtshof (2003b), Rn. 
Trotz des EuGH-Urteils zur Festbetragssetzung durch die Spitzenverbände der Krankenkassen sind deshalb in Zukunft auch von den kartellrechtlichen Vorschriften des EGV Auswirkungen auf die nationalen Gesundheitssysteme zu erwarten. Bei zukünftigen Reformen wird insbesondere darauf geachtet werden, die gesetzlichen Krankenversicherungen nicht soweit von ihren „,sozialen“ Eigenschaften zu entkleiden, dass sie sogar bei der Erfüllung ihrer Hauptaufgabe als Unternehmen gelten.

Unabhängig von der Geltung des europäischen Wettbewerbs- und Kartellrechts findet jedoch das europäische Vergaberecht Anwendung im Bereich der GKV, wenn die Krankenkassen als öffentliche Auftraggeber mit Leistungserbringern entgeltliche Verträge schließen und der Wert des Auftrags die Schwelle von $200.000 €$ überschreitet. ${ }^{169}$ Treffen diese Bedingungen zu, so besteht eine Verpflichtung zur öffentlichen Ausschreibung, die das Zustandekommen von Verträgen aus verwaltungstechnischen Gründen erheblich erschweren kann. Eine Anwendung des Vergaberechts kommt jedoch im Bereich der Zulassung von Leistungserbringern zur GKV-Versorgung nur dann in Betracht, wenn die Kassen hier eine bindende Vorauswahl treffen, da nur dann ein öffentlicher Auftrag vorliegt. So bleibt dieses Recht beispielsweise beim Abschluss von Verträgen zur integrierten Versorgung außen vor, da hier letztlich die Patienten die eigentliche Nachfrageentscheidung treffen. Wahrscheinlicher ist seine Anwendung im Bereich der Hilfsmittel.

\subsection{Offene Methode der Koordinierung}

Die Osterweiterung, die stärkere Öffnung nationaler Wirtschaftsräume sowie die politische Vertiefung der Europäischen Union machten aufgrund der zunehmenden Wanderungsbewegungen zwischen den Staaten eine stärkere Koordinierung der nationalen Sozialpolitiken notwendig. Eine Harmonisierung der nationalen Politiken auf europäischer Ebene scheidet jedoch aus, da die alleinige Kompetenz zur Ausgestaltung der Systeme der sozialen Sicherheit bei den Mitgliedstaaten liegt. Der Europäische Rat von Lissabon beschloss deshalb auf seiner Frühjahrstagung 2000, die sog. „offene Methode der Koordinierung" (OMK) im Bereich der sozialen Sicherung einzusetzen. Sie soll dazu beitragen, „die Union zum wettbewerbsfähigsten und dynamischsten wissensbasierten Wirtschaftsraum der Welt zu machen - einem Wirtschaftsraum, der fähig ist, ein dauerhaftes Wirtschaftswachstum mit mehr und besseren Arbeitsplätzen und einem größeren sozialen Zusammenhalt zu erzielen"170

Die OMK zielt darauf ab, eine Verbreitung bewährter Praktiken und eine größere Konvergenz in Bezug auf die wichtigsten Ziele der EU zu erreichen. ${ }^{171}$ Dabei bietet sie den Mitgliedstaaten eine Hilfe bei der schrittweisen Entwicklung ihrer eigenen Politiken. Die Umsetzung der OMK sieht folgende Komponenten vor: ${ }^{172}$

\footnotetext{
${ }^{169} \mathrm{Vgl}$. im Folgenden Koenig/Engelmann/Hentschel (2003), S. $565 \mathrm{ff}$.

${ }^{170}$ Europäischer Rat (2000), Rn. 5.

171 Vgl. Europäischer Rat (2000), Rn. 37.

${ }^{172} \mathrm{Vgl}$. Europăische Kommission (2003d), S. $10 \mathrm{f}$.
} 
- Die Vertreter der Mitgliedstaaten vereinbaren Leitlinien, die jeweils einen genauen Zeitplan für die Verwirklichung der von ihnen gesetzten kurz-, mittel- und langfristigen Ziele beinhalten.

- Es werden quantitative und qualitative Indikatoren und Benchmarks vereinbart, die auf die Bedürfnisse der Mitgliedstaaten zugeschnitten sind. Sie dienen dem Vergleich der unterschiedlichen Strategien der Staaten, um so bewährte Praktiken, die sog. „,best practices”, vergleichen zu können.

- Die Umsetzung der Leitlinien erfolgt durch die Entwicklung konkreter Ziele und den Erlass entsprechender Maßnahmen unter Berücksichtigung der nationalen und regionalen Unterschiede.

- Der Prozess beinhaltet regelmäßige Überwachungen, Bewertungen und gegenseitige Überprüfungen der Politiken der Mitgliedstaaten in Bezug auf die Erreichung der Ziele, ohne dass für den Fall schlechter Bewertungen Sanktionsmöglichkeiten vorsehen sind.

Die aufgeführten Komponenten geben einen Rahmen für die Anwendung der Methode in den verschiedenen Bereichen vor. Bei ihrer konkreten Anwendung bedarf es jeweils einer Entscheidung darüber, wie sie im Einzelnen ausgestaltet wird, welche Arbeitsverfahren Anwendung finden und wie die Organisation der Arbeiten erfolgt. Der Europäische Rat erhofft sich von der Anwendung der Methode eine Verbesserung der bestehenden Prozesse und sieht für sich eine stärkere Leitungs- und Koordinierungsfunktion. ${ }^{173}$

Die OMK ist von ihrer Konzeption her durch ein relativ geringes Maß an Verbindlichkeit für die Mitgliedstaaten gekennzeichnet. ${ }^{174}$ Sie dient dem systematischen Austausch und Vergleich von Problemen und Lösungsstrategien und soll auf diese Weise einen gemeinsamen EU-weiten Lernprozess initiieren. Damit stellt sie einen Mittelweg zwischen einer europaweiten Harmonisierung der Sozialpolitiken und einer vollständig unabhängigen nationalstaatlichen Gestaltung und Steuerung dar. Die Anwendung der OMK erfolgt in Politikbereichen, in denen die EU lediglich über begrenzte Befugnisse verfügt und darum wenig direkten Einfluss auf die Politiken der Mitgliedstaaten nehmen kann. Um das Subsidiaritätsprinzip zu wahren, das für diese Bereiche gilt, bleiben Auswahl und Umsetzung der nationalen Maßnahmen zur Erreichung der im Rahmen der OMK vom allen Mitgliedstaaten gemeinsam erarbeiteten Ziele den nationalen Regierungen überlassen. ${ }^{175}$ Es wird ein dezentraler Ansatz angestrebt, der es der EU, den Mitgliedstaaten, den regionalen und lokalen Ebenen sowie den Sozialpartnern und der Bürgergesellschaft ermöglichen soll, im Rahmen unterschiedlicher Formen von Partnerschaften zu agieren und an der Diskussion über gemeinsame Ziele, Indikatoren und Benchmarks mitzuwirken. ${ }^{176}$ Die Methode zielt zudem darauf ab, durch regelmäßige Überprüfungen und Vergleiche der Zielerreichung in den Mitgliedstaaten trotz der feh-

\footnotetext{
${ }^{173}$ Vgl. Europäischer Rat (2000), Rn. 7.

${ }^{174} \mathrm{Vgl}$. Schmucker (2003a), S. 206.

${ }^{175}$ Vgl. Art. 5 EGV.

${ }^{176}$ Vgl. Europäische Kommission (2003d), S. 12.
} 
lenden Sanktionsmöglichkeiten einen gewissen Druck auf die Regierungen auszuüben, eventuelle negative Ergebnisse zu korrigieren.

Die OMK wurde in Fragen der sozialen Sicherung zunächst in den Bereichen „Soziale Eingliederung und Bekämpfung der Armut”, „Renten” sowie „bessere Arbeitsplätze” (Arbeitsschutz) eingesetzt. Auf seiner Tagung im Juni 2001 in Göteborg übertrug der Europäische Rat der Kommission dann die Aufgabe, einen ersten Bericht über Leitlinien im Bereich des Gesundheitswesens und der Altenpflege zu erstellen, ${ }^{177}$ den diese im Dezember vorlegte. ${ }^{178}$ Er kann als erster Schritt zur Anwendung der OMK im Gesundheitswesen gesehen werden. Darin postuliert die EU-Kommission die gemeinsame Herausforderung für alle Mitgliedstaaten, gleichzeitig

- einen allgemeinen Zugang zu medizinischen Leistungen,

- eine qualitativ hochwertige medizinische Versorgung sowie

- die langfristige Finanzierbarkeit des Gesundheitswesens zu sichern. ${ }^{179}$

In ihrer Mitteilung vom April 2004 schlug die Kommission vor, die Strategie der „Modernisierung der Sozialschutzsysteme“ um eine OMK im Bereich der „Gesundheitsversorgung und Langzeitpflege" $\mathrm{zu}$ erweitern. ${ }^{180}$ In diesem Bericht erarbeitete sie zudem konkretere Vorschläge, wie die gemeinsamen Ziele einer hochwertigen, allgemein zugänglichen und zukunftsfähigen Gesundheitsversorgung und Langzeitpflege zur realisieren sind. ${ }^{181}$

Bereits auf seiner Frühjahrstagung 2003 beschloss der Europäische Rat, die OMKProzesse im Bereich des Sozialschutzes zusammenzuführen. Als Ziele dieses sog. „Streamlining“ nennt die EU-Kommission eine effizientere Gestaltung der Zusammenarbeit der Akteure sowie die Nutzung von Synergien zwischen den verschiedenen Arbeitssträngen. ${ }^{182}$ Die Definition eines einzigen Bündels gemeinsamer Ziele in den Bereichen soziale Eingliederung, Renten sowie Gesundheitsversorgung und Langzeitpflege, das im März 2006 vom Europäischen Rat verabschiedet wurde und an die Stelle der bestehenden separaten Zielbündel trat, soll dieses Anliegen umsetzen. Innerhalb dieses neuen Bündels gibt es drei übergreifende Ziele, die einen allgemeinen Rahmen für die OMK-Arbeiten vorgeben sollen, sowie jeweils drei Ziele in den drei Themenbereichen.183 Die Ziele für den Themenbereich Gesundheit bleiben die Sicherung einer allgemeinen Zugänglichkeit zur Versorgung, die Sicherung der Qualität sowie die nachhaltige Finanzierbarkeit der Gesundheitsversorgung und Pflege. Die Kommission formulierte sie in ihrer Mitteilung jedoch etwas konkreter aus.

\footnotetext{
${ }^{177}$ Vgl. Europäischer Rat (2001), Rn. 43.

${ }^{178} \mathrm{Vgl}$. Europäische Kommission (2001).

${ }^{179} \mathrm{Vgl}$. Europäische Kommission (2001), S. 15.

${ }^{180} \mathrm{Vgl}$. Europäische Kommission (2004b).

${ }^{181} \mathrm{Vgl}$. Europäische Kommission (2004b) sowie Tabelle I im Anhang A.

${ }^{182} \mathrm{Vgl}$. Europäische Kommission (2003d), S. 5.

${ }^{183} \mathrm{Vgl}$. Europäische Kommission (2005e), S. 6. Die in der Mitteilung genannten Ziele sind in Tabelle II im Anhang A aufgefuhrt.
} 
Die Straffung der OMK im Sozialschutz soll dazu beitragen, eine bessere Integration dieses Bereiches in den Lissabon-Prozess ${ }^{184}$ und dabei insbesondere in die Grundzüge der Wirtschaftspolitik und die Europäische Beschäftigungsstrategie (EBS) zu erreichen. ${ }^{185}$ Aus diesem Grund forderte die Kommission, dass sich das neue Bündel der gemeinsamen sozialpolitischen Ziele in Einklang mit den zu verabschiedenden Leitlinien zur Wirtschafts- und Beschäftigungspolitik befindet und eine enge Verzahnung mit diesen aufweist. ${ }^{186}$ Den ersten gemeinsamen Sozialschutzbericht erstellte die Kommission auf Basis der von den Mitgliedstaaten erarbeiteten Berichte in einer Übergangsform im Jahre 2005 mit einem thematischen Schwerpunkt auf die Fragen der sozialen Eingliederung. ${ }^{187}$ Im Jahre 2006 beschäftigte sich der Bericht dann mit der Umsetzung der von der Kommission erarbeiteten übergreifenden Ziele und ging kurz auf die Fortschritte der OMK in den drei Teilbereichen ein. ${ }^{188}$ Dieser gemeinsame Sozialschutzbericht stellt das zentrale Instrument des neuen, gestrafften Prozesses dar und dient dazu, die Fortschritte der offenen Koordinierung der Sozialschutzpolitik und der Eingliederungspolitik zu dokumentieren und zu bewerten. ${ }^{189}$ In den Jahren 2007 und 2008 dienen die Berichte hauptsächlich zur Darlegung der Fortschritte bei der Durchführung der gewählten Strategie. Dieser Zyklus soll in den Folgejahren beibehalten werden, so dass alle drei Jahre ein umfassender, zukunftsweisender Bericht erstellt wird. Im Jahre 2009 ist damit der Beginn der zweiten Runde der OMK im Bereich Sozialschutz mit der Festlegung überarbeiteter und validierter gemeinsamer Ziele durch den Rat vorgesehen.

Im Juni 2006 legte der Ausschuss für Sozialschutz (Social Protection Committee, SPC) einen Bericht vor, der die Indikatoren beinhaltet, auf deren Grundlage die Mitgliedstaaten bis zum September 2006 ihre nationalen Berichte erstellen, die wiederum die Basis für einen umfassenden Bericht der Kommission an den Europäischen Rat für dessen Tagung im Frühjahr 2007 bilden. ${ }^{190}$ Die Indikatoren setzen sich zusammen aus übergreifenden Indikatoren zum Sozialschutz sowie speziellen Indikatoren für jeden der drei in die OMK einbezogenen Teilbereiche. Der Sozialausschuss weist für die Indikatoren zum Bereich "Gesundheitsversorgung und Langzeitpflege" ausdrücklich darauf hin, dass hier bisher die Arbeiten noch nicht abgeschlossen sind und darum die vorgestellten Vorschläge wahrscheinlich nicht vollständig mit der späteren Indikatorenliste für die OMK übereinstimmen. Zu den drei Themen „Zugang zur Gesundheitsversorgung“, „Qualität" und ,nachhaltige Finanzierbarkeit" sind sowohl übergreifende als auch themenbezogene Gesundheitsindikatoren vorgeschlagen, von den bisher 20 Indikatoren erfasst jedoch nur einer die Qualitätsdimension.

\footnotetext{
${ }^{184}$ Der Lissabon-Prozess (auch Lissabon-Strategie oder Lissabon-Agenda) ist ein auf der Frühjahrstagung der europäischen Staats- und Regierungschefs im März 2000 in Lissabon verabschiedetes Programm, dessen Ziel es ist, die EU bis 2010 zum wettbewerbsfähigsten und dynamischsten wissensbasierten Wirtschaftsraum der Welt zu machen.

${ }^{185} \mathrm{Vgl}$. Europäische Kommission (2005e), S. 3.

${ }^{186} \mathrm{Vgl}$. Europäische Kommission (2003d), S. 6.

${ }^{187} \mathrm{Vgl}$. Europäische Kommission (2005b).

${ }^{188} \mathrm{Vgl}$. Europäische Kommission (2006a).

${ }^{189} \mathrm{Vgl}$. auch im Folgenden Europäische Kommission (2003d), S. $18 \mathrm{ff}$.

190 Vgl. Europăische Kommission (2006c).
} 
Bisher wurde die OMK im Bereich der Gesundheitsversorgung und Langzeitpflege mit einiger Zurückhaltung betrieben. ${ }^{191}$ Dies kann darauf zurückzuführen sein, dass die Staats- und Regierungschefs im Europäischen Rat als handlungstragende Personen einer Überprüfung der Ergebnisse ihrer nationalen Politiken kritisch gegenüberstehen. ${ }^{192}$ Sundmacher (2005b, S. 24f) geht hingegen davon aus, dass gerade die Mitgliedstaaten mittels ihres Einflusses im Europäischen Rat eine Zentralisierung der Sozialpolitik auf europäischer Ebene mit dem Einsatz der OMK fördern wollen, weil sie sich daraus politische Vorteile versprechen: $\mathrm{Da}$ die nationalen Sozialpolitiken anders als in vergangenen Jahrzehnten nur noch eingeschränkt zum Gewinn von Wählerstimmen beitragen, sondern aufgrund der notwendigen Reformmaßnahmen ein erhebliches politisches Risiko darstellen, ist es für die nationalen Regierungen rational, die Kompetenz für diese ungeliebten Einschränkungen auf die europäische Ebene zu verlagern. Für eine solche Vorgehensweise sprächen auch die Vorschläge der High Level Reflection Group (2003), die sich vor allem aus hochrangigen Vertretern der Mitgliedsiänder (aus Deutschland z.B. Gesundheitsministerin Ulla Schmidt) zusammensetzt und eine stärkere Kompetenzverlagerung in der Gesundheitspolitik bis hin zur Reform des einschlägigen Art. 152 EGV erwägt. ${ }^{193}$ Die Zurückhaltung beim Einsatz der OMK geht laut Sundmacher von der Europäischen Kommission aus, die umfangreiche Möglichkeiten des Rates zur Einflussnahme auf die Einführung und Ausgestaltung der Methode kritisch sieht und einen Machtverlust befürchtet. ${ }^{194}$

Obwohl die nationalen Akteure im deutschen Gesundheitswesen den Einsatz der OMK mit dem Ziel einer engeren Zusammenarbeit der Mitgliedstaaten und einer stärkeren Koordinierung der nationalen Politiken grundsätzlich begrüßen, ${ }^{195}$ gibt es zahlreiche skeptische Stimmen. Diese üben sowohl an der Umsetzung der Methode im Allgemeinen als auch an dem von der Kommission vorgestellten Streamlining Kritik, die sich insbesondere auf drei Punkte bezieht: ${ }^{196}$

- Gefahr eines Demokratiedefizits,

- Tendenz zur Überbetonung fiskalischer Aspekte,

- Probleme bei Indikatorenbildung und Benchmarking.

Eine mangelnde demokratische Legitimation wird von den Kritikern sowohl auf europäischer als auch auf nationaler Ebene gesehen. Da der Ministerrat die gemeinsamen Ziele für den Koordinierungsprozess auf Vorschlag der Europäischen Kommission festlegt und eine Einbeziehung des Europäischen Parlaments in den Prozess derzeit nicht vorgesehen ist, ${ }^{197}$ bleibt das einzige demokratisch gewählte Gremium auf europäischer Ebene bei der Erarbeitung und Umsetzung außen vor. Zudem erhalten auf der

\footnotetext{
${ }^{191}$ So auch Schulte (2005), S. 21.

${ }^{192}$ Diese Auffassung vertreten z.B. Heidenreich/Bischoff (2006), S. 13 f.

${ }^{193} \mathrm{Vgl}$. Europäische Kommission (2003f), S. $10 \mathrm{ff}$.

${ }^{194} \mathrm{Vgl}$. Sundmacher (2005b), S. 24f.

${ }^{195} \mathrm{Vgl}$. Arbeitsgemeinschaft der Spitzenverbände der gesetzlichen Krankenkassen (2002), S. 4; Tiemann (2004), S. 96.

${ }^{196}$ Vgl. u.a. Gesellschaft für Versicherungswissenschaft und -gestaltung (GVG) (2002).

${ }^{197}$ Vgl. Arbeitsgemeinschaft der Spitzenverbände der gesetzlichen Krankenkassen (2002), S. 5.
} 
Ebene der Mitgliedstaaten die nichtstaatlichen Akteure sowie die regionalen Verwaltungs- und Regierungsebenen kaum Gelegenheit, an der Zielbestimmung und der Analyse der nationalen Gegebenheiten mitzuarbeiten. Darum besteht die Gefahr, de facto einen EU-Standard zu definieren, der nicht berücksichtigt, dass in der unterschiedlichen Ausgestaltung der sozialen Sicherungssysteme auch die Präferenzen der betroffenen Bürger zum Ausdruck kommen. Zudem wird vielfach betont, dass der Einsatz der OMK nicht die Kompetenz der Mitgliedstaaten für die Gesundheitspolitik aushöhlen darf, auch wenn der Europäische Rat und die Kommission durch sie neue strategische Kompetenzen in diesem Bereich erhalten. ${ }^{198}$ Hier ist zu beobachten, dass der Rat eine Mitarbeit der verschiedenen gesellschaftlichen Gruppen am Prozess der Zielbildung eher behindert, ${ }^{199}$ obwohl die Kommission eine stärkere Beteiligung der sektoralen Vertragspartner auf europäischer Ebene als wünschenswert ansieht. ${ }^{200}$ Allerdings dürfte eine solche Einbeziehung weiterer Akteure zu Problemen mit dem derzeit sehr engen Zeitplan führen.

Darüber hinaus besteht die Befürchtung, dass die geplante Abstimmung des Koordinierungsprozesses im Bereich Sozialschutz mit den Instrumenten zur Koordinierung der Wirtschafts- und Beschăftigungspolitik die Konzentration auf die Finanzierungsseite des Gesundheitswesens fördert und qualitative Aspekte der Versorgung in den Hintergrund rückt. ${ }^{201}$ Diese Tendenz könnten datentechnische Probleme verstärken, die sich daraus ergeben, dass die Entwicklung in den sozialpolitischen Bereichen nur sehr eingeschränkt durch quantitative Indikatoren abbildet werden kann und die notwendigen qualitativen Daten oftmals kaum verfügbar sind.

Ohnehin ergeben sich bei der Indikatorenbildung sowie bei der Erhebung der benötigten Daten große konzeptionelle Schwierigkeiten, die den Einsatz der Methode im Gesundheitsbereich grundsätzlich in Frage stellen. Um den Grad der Realisierung der vorher vereinbarten Ziele in den einzelnen Mitgliedstaaten abzubilden bzw. zu messen, ist die Erarbeitung unionsweit einheitlicher objektivierbarer Indikatoren vorgesehen. ${ }^{202}$ Erfahrungen aus internationalen Gesundheitssystemvergleichen (z.B. von der World Health Organization (WHO) und der Organisation for Economic Cooperation and Development (OECD)) zeigen jedoch, dass die Datenlage für einen europaweiten Systemvergleich unzureichend ist, da in den Mitgliedstaaten zum Teil Datenlücken und Mängel in der Qualität der erhobenen Daten bestehen. ${ }^{203}$ Obwohl auf diesem Gebiet in den vergangenen Jahren erhebliche Anstrengungen unternommen wurden, mangelt es den erhobenen Daten oftmals an Vergleichbarkeit. Da sich die sehr unterschiedliche

\footnotetext{
198 Vgl. u.a. Bundesministerium für Gesundheit und Soziale Sicherung (BMGS) (2005c); Deutscher Bundesrat (2004); Gesellschaft für Versicherungswissenschaft und -gestaltung (GVG) (2004), S. 6; Orosz (2004), S. 24; Arbeitsgemeinschaft der Spitzenverbănde der gesetzlichen Krankenkassen (2004), S. 32. Schmucker (2003a), S. 209.

${ }^{199} \mathrm{Vgl}$. Heidenreich/Bischoff (2004), S. 11.

${ }^{200} \mathrm{Vgl}$. Europäische Kommission (2004b), S. 8.

${ }^{201} \mathrm{Vgl}$. Gesellschaft für Versicherungswissenschaft und -gestaltung (GVG) (2003), S. 6 f.

${ }^{202} \mathrm{Vgl}$. Arbeitsgemeinschaft der Spitzenverbände der gesetzlichen Krankenkassen (2002), S. 6.

${ }^{203} \mathrm{Vgl}$. Arbeitsgemeinschaft der Spitzenverbände der gesetzlichen Krankenkassen (2002), S. 8ff; Gesellschaft für Versicherungswissenschaft und -gestaltung (GVG) (2002), S. 7.
} 
Ausprägung der nationalen Gesundheitssysteme grundsätzlich negativ auf diese Vergleichbarkeit auswirkt, werden die damit verbundenen methodischen Probleme auch langfristig bestehen bleiben. Die Interpretation erfassbarer Strukturmerkmale besitzt darum oftmals nur dann eine ausreichende Aussagekraft, wenn sie die Eigenschaften des jeweiligen Systems mit einbezieht. ${ }^{204}$ Neben diesen grundsätzlichen datentechnischen Schwierigkeiten im Gesundheitsbereich weckt die Forderung der Kommission nach einer möglichst geringen Gesamtzahl der Indikatoren ${ }^{205}$ die Befürchtung, dass das Benchmarking der verschiedenen Gesundheitssysteme kein adäquates Bild wiedergeben wird und auf diese Weise die Erkenntnisse und Empfehlungen verzerrt.

Die skizzierten Faktoren tragen dazu bei, dass aus heutiger Sicht eine generelle Beurteilung der zukünftigen Ausgestaltung der Methode und ihre Auswirkungen auf die nationalen Systeme nicht möglich ist. Die Reaktionen auf den Weltgesundheitsbericht der WHO ${ }^{206}$ im Jahre 2000, der dem deutschen Gesundheitssystem unter 191 Ländern lediglich Platz 25 zuwies, zeigten jedoch, dass derartige Benchmarking-Prozesse Diskussionen in den einzelnen Ländern über die Effizienz und Effektivität des nationalen Gesundheitswesens auslösen können. ${ }^{207} \mathrm{Da}$ auf europäischer Ebene in der Gesundheitspolitik derzeit ein Gestaltungsvakuum besteht, das in den vergangenen Jahren zunehmend vom Europäischen Gerichtshof durch die Anwendung des Binnenmarkt- und Wettbewerbsrechts auf die nationalen Gesundheitssysteme gefüllt wurde, könnte der OMK die positive Rolle zukommen, qualitative Gesundheitsziele und -indikatoren zu definieren, auf deren Basis sich die europäische Gesundheitspolitik in Zukunft vom bloßen Anhängsel der Binnenmarktintegration zum eigenständigen Gemeinschaftsziel entwickelt. ${ }^{208}$ Aufgrund der Komplexität des Systems erscheint jedoch die Empfehlung von Sundmacher (2005a, S. 208) sinnvoll, die Methode auf Teilbereiche anzuwenden und statt einer sehr breiten eher eine vertiefte Anwendung vorzunehmen, z.B. indem sich die Bearbeitung jährlich auf bestimmte Schwerpunktthemen beschränkt, die in einen längeren Turnus regelmäßig wieder aufgenommen werden. Offenbar ist eine ähnliche Vorgehensweise im Gesundheitsbereich für den ersten Zyklus der Berichterstattung und Bewertung im Rahmen der OMK vorgesehen. Die nationalen Berichte der Mitgliedstaaten, die im September 2006 vorgelegt werden sollten, enthalten neben allgemeineren Teilen auch fachspezifische Pläne für die Teilbereiche. Dabei soll sich der Plan für die Gesundheitsversorgung und Langzeitpflege ,auf die Ermittlung von Einzelfragen konzentrieren, bei denen sich ein gegenseitiger Austausch und gegenseitiges Lernen anbieten “209. Inwieweit sich daraus eine tatsächliche Koordinierung der mit-

${ }^{204}$ Ein Beispiel bilden die im internationalen Vergleich hohen Arzneimittelausgaben in Frankreich. Um dieses Ergebnis beurteilen zu können, bedarf es einer Analyse, ob und inwieweit diese Aufwendungen zu Einsparungen in anderen Sektoren füren, z.B. im stationären Sektor durch eine geringere Zahl von Krankenhauseinweisungen.

${ }^{205}$ Vgl. Europäische Kommission (2003d), S. 16.

${ }^{206} \mathrm{Vgl}$. World Health Organization (WHO) (2000).

${ }^{207} \mathrm{Vgl}$. Weber/Brenner/Weisner (2001), S. A2932ff.

${ }^{208} \mathrm{Vgl}$. Schmucker (2003a), S. 209.

${ }^{209}$ Europäische Kommission (2005e), S. 8. 
gliedstaatlichen Politiken oder gar eine Konvergenz der Gesundheitssysteme ergeben wird, bleibt abzuwarten.

\subsection{Zwischenfazit}

Die EU nimmt explizit und implizit auf verschiedenen Wegen Einfluss auf die nationalen Gesundheitssysteme und -politiken. Auch wenn aufgrund des Subsidiaritätsprinzips keine einheitliche europäische Gesundheitspolitik existiert, begann die Entwicklung der Kompetenzen der $E U$ im Gesundheitsbereich bereits mit der Unterzeichnung des Vertrages über die Gründung der Europäischen Gemeinschaft für Kohle und Stahl (EGKS) im April 1951. Sie vollzog sich in verschiedenen Phasen, wobei der derzeit letzte Schritt in der Anwendung der OMK im Themenbereich Gesundheit zu sehen ist. Mit dem Maastrichter Vertrag erhielt die EU Einflussnahmemöglichkeiten im Bereich der Prävention und bei der Sicherstellung eines hohen Gesundheitsschutzniveaus. Die von der Kommission seit 1993 aufgelegten und vorgeschlagenen Aktionsprogramme zeigen deutlich, dass sie den Willen hat, diese Kompetenzen zu nutzen. In zunehmendem Maße unternimmt sie dabei den Versuch, auf diesem Weg Einfluss auf die Organisation der Gesundheitssysteme zu gewinnen. Bisher wirken die von der EU auf Basis dieser Zuständigkeiten ergriffenen Maßnahmen zwar direkt auf verschiedene Politikfelder mit Gesundheitsbezug, lassen dabei aber die nationalen Gesundheitssysteme weitgehend unberührt. Es sind vielmehr vorwiegend die Regelungen zur Verwirklichung eines einheitlichen Binnenmarktes, die Einfluss auf die Organisation der Systeme nehmen. Hierzu zählen die vier Grundfreiheiten des EGV, die sich aus den sachbezogenen Freiheiten des Waren- und Kapitalverkehr und den personenbezogenen Freiheiten des Dienstleistungs- und Personenverkehrs zusammensetzen, sowie das europäische Wettbewerbsrecht.

Im Rahmen des Gesundheitswesens ist die Personenverkehrsfreiheit in ihrer Ausprägung als Niederlassungsfreiheit für die Mitglieder derjenigen Berufe relevant, die in selbstständiger Tätigkeit ausgeübt werden - hierzu zählen beispielsweise niedergelassene Ärzte und Apotheker. Für sie besteht aufgrund dieser Freiheit die Möglichkeit, ihrer Tätigkeit dauerhaft in einem anderen EU-Mitgliedstaat nachzugehen. Im Rahmen dieser Arbeit ist diesbezüglich beispielsweise von Interesse, inwieweit die in Deutschland praktizierte Bedarfsplanung im Bereich der vertragsärztlichen Versorgung gegen diese Niederlassungsfreiheit verstößt, wenn sie Mediziner mit Staatsangehörigkeiten anderer EU-Staaten oder ausländischen Examen an der Aufnahme einer selbständigen Tätigkeit hindert. $\mathrm{Zu}$ dieser Frage existieren in der Literatur divergierende Ansichten. $\mathrm{Da}$ der EuGH auf diese Frage bisher nicht eingegangen ist, besteht hier keine rechtliche Klarheit.

Abhängig beschäftigten EU-Bürgern gewährt die Arbeitnehmerfreizügigkeit das Recht, unionsweit eine Arbeit aufzunehmen. Bereits zu Beginn der europäischen Integrationsbemühungen wurde berücksichtigt, dass die Koordinierung der sozialen Sicherungssysteme der einzelnen Mitgliedstaaten eine wichtige Voraussetzung für die Wahrnehmung des Rechts auf Freizügigkeit darstellt. Das koordinierende Sozialrecht regelt in den Verordnungen 1408/71/EWG und 571/72/EWG in Bezug auf Leistungen 
bei Krankheit die Versicherungszugehörigkeit von sog. Wanderarbeitnehmern sowie die Möglichkeit der Leistungsinanspruchnahme bei vorübergehendem Auslandsaufenthalt. Lange Zeit stellte eine Inanspruchnahme auf Basis dieser Regelungen die einzige Möglichkeit dar, eine grenzüberschreitende Kostenübernahme der Krankenkasse zu erreichen. Außer in Notfällen bedurfte es hierzu einer vorherigen Genehmigung des zuständigen Trägers, die nur unter relativ restriktiven Bedingungen erteilt werden konnte. Die auf Basis dieser Regelungen durchgeführten Behandlungen nahmen und nehmen darum lediglich einen verschwindend geringen Anteil an allen von der GKV getragenen Leistungen ein.

Eine weitere Möglichkeit zur Kostenübernahme durch die Krankenkassen bei einer grenzüberschreitenden Leistungsinanspruchnahme schufen im ambulanten Bereich die EuGH-Urteile in den Rechtssachen „Kohll" und „Decker" sowie die darauf aufbauende Rechtsprechung. Sie räumen EU-Bürgen das Recht ein, im Rahmen der Dienstleistungs- bzw. Warenverkehrsfreiheit ausländische Leistungserbringer außerhalb von Krankenhäusern aufzusuchen und nachträglich die Kosten von den Krankenkassen erstattet zu bekommen. Sollte sich darüber hinaus erweisen, dass die „Kohll/Decker“Rechtsprechung auch zur Bewertung des Verhältnisses zwischen den nationalen Krankenversicherungssystemen und derjenigen ausländischen Leistungserbringern heranzuziehen ist, die Leistungen nur vorübergehend im Inland erbringen, könnte dies dazu führen, dass im Fall einer Inanspruchnahme von Gesundheitsleistungen bei diesen ausländischen Anbietern eine erleichterte Kostenübernahme durch die nationalen Krankenversicherungen möglich wird. Damit würde die territoriale Begrenzung der Leistungserbringung auf das jeweilige Land der Niederlassung deutlich aufgeweicht.

Die Warenverkehrsfreiheit spielt im Gesundheitswesen aber nicht nur bei der grenzüberschreitenden Inanspruchnahme von Leistungen, sondern auch in Bezug auf zahlreiche Regelungen im Arzneimittelbereich eine große Rolle. So wurde das europaweit geltenden Zulassungsverfahren, das die Marktzulassung für Arzneimittel in der EU erleichtert, zur Stärkung des freien Arzneimittelverkehrs eingeführt. Zudem schützt die Warenverkehrsfreiheit entsprechend der Rechtsprechung des EuGH den Parallel- und Reimport von Arzneimitteln und setzt damit vor allem auch patentgeschützte Arzneimittel einem stärkeren Preisdruck aus. Wie in Abschnitt 3.3.1 ausgeführt, ergibt sich aufgrund der zahlreichen Regulierungen in den Mitgliedstaaten daraus ein Spannungsverhältnis zwischen freiem Warenverkehr innerhalb der EU und den nationalen Preisregulierungen, die in allen europäischen Mitgliedstaaten direkt oder indirekt zur Anwendung kommen.

Neben den vier Grundfreiheiten bildet die Regelungen des europäischen Wettbewerbsrechts ein weiteres Kernstück zur Verwirklichung des unionsweiten Binnenmarkts. Diese Regelungen spielen bei der Beurteilung der Unternehmenseigenschaften von gesetzlichen Krankenkassen eine Rolle. Der EuGH wertete die Krankenkassen in seinem viel beachteten Urteil zur Festsetzung von Arzneimittelfestbeträgen durch die Krankenkassenverbände in Deutschland sowohl in der Erfüllung ihrer Haupttätigkeit als auch bei der Festsetzung der Festbeträge nicht als Unternehmen. Dennoch kommt heute schon in Betracht, dass die Krankenkassen - beispielsweise beim Angebot integrierter Versorgungsformen - wirtschaftliche Tätigkeiten ausüben und somit in diesem Be- 
reich Unternehmenseigenschaften aufweisen können. Zudem könnte bei einer weiteren Stärkung der Wettbewerbselemente und einer Vergrößerung der Spielräume einzelner Kassen hinsichtlich ihres Leistungsangebots auch die Haupttätigkeit der Kassen als wirtschaftlich einzustufen sein. Es ist deshalb zu erwarten, dass in Zukunft auch von den wettbewerbsrechtlichen Vorschriften des EGV verstärkt Auswirkungen auf die nationalen Gesundheitssysteme ausgehen. Allerdings ergeben sich hier - anders als durch die Anwendung der Grundfreiheiten auf das Gesundheitswesen - wahrscheinlich keine liberalisierenden Tendenzen. Vielmehr ist damit zu rechnen, dass die drohende Anwendung des Wettbewerbsrechts tendenziell zur Zurückhaltung bei der Einführung wettbewerblicher Elemente in die deutsche Kassenlandschaft führen wird.

Eine Konsequenz aus den Urteilen des EuGH zur grenzüberschreitenden Kostenübernahme ist ein Bedeutungszuwachs der Gesundheitspolitik auf europäischer Ebene, weil durch die Möglichkeit einer größeren Patientenwanderung der Wunsch nach einer stärkeren Koordinierung der Gesundheitssysteme entstand. Da ein direktes Eingreifen in die Organisation der nationalen Gesundheitssysteme aufgrund des Subsidiaritătsprinzips nicht möglich ist, entschied sich der Europäische Rat, die sog. „offene Methode der Koordinierung " (OMK) im Bereich der Gesundheitsversorgung einzusetzen. Die OMK dient dem systematischen Austausch und Vergleich von Problemen und Lösungsstrategien im Rahmen von Benchmark-Prozessen und soll auf diese Weise einen gemeinsamen EU-weiten Lernprozess initiieren. Damit stellt sie einen Mittelweg zwischen einer europaweiten Harmonisierung der Sozialpolitiken und einer vollständig unabhängigen nationalstaatlichen Gestaltung und Steuerung dar. Die Methode ist von ihrer Konzeption her durch ein relativ geringes $\mathrm{Ma}$ an Verbindlichkeit für die Mitgliedstaaten gekennzeichnet. Ihr Einsatz erfolgt in Politikbereichen, in denen die EU lediglich über begrenzte Befugnisse verfügt. Im Gesundheitsbereich befindet sich die Anwendung der Methode noch in der Anfangsphase und ist mit Problemen insbesondere bei der Indikatorenbildung behaftet, so dass aus heutiger Sicht eine generelle Beurteilung der zukünftigen Ausgestaltung der Methode und ihrer Auswirkungen auf die nationalen Systeme nicht möglich ist. Bei einer günstigen Entwicklung könnte der OMK jedoch die positive Rolle zukommen, qualitative Gesundheitsziele und -indikatoren zu definieren, auf deren Basis sich eine aktive europäische Gesundheitspolitik entwickelt, die nicht nur indirekt durch die Binnenmarktintegration vorangetrieben wird.

Die Darstellung der Einflussfaktoren der Europäischen Union auf die Gesundheitsversorgung der Mitgliedstaaten zeigt, dass diese Faktoren seit Beginn der Integrationsbemühungen bestehen und in den vergangenen Jahren und Jahrzehnten stark an Bedeutung gewonnen haben. Von besonderem öffentlichem Interesse war dabei die Rechtsprechung des EuGH zur grenzüberschreitenden Kostenübernahme bei Gesundheitsleistungen. Doch auch die anderen dargestellten direkten und indirekten Einflussfaktoren werden in den kommenden Jahren an Bedeutung gewinnen und in zunehmendem Maße auf die Organisation der nationalen Gesundheitssysteme einwirken. Diese allein als Gefahr für die nationalen Kompetenzen oder gar die finanzielle Steuerungsfähigkeit des Systems zu sehen, entspricht einer sehr pessimistischen Sichtweise, die unberücksichtigt lässt, dass diese Entwicklungen das Potential besitzen, Ineffizienzen und 
Ineffektivitäten in den nationalen Gesundheitssystemen $\mathrm{zu}$ identifizieren und $\mathrm{zu}$ verringern. Von besonderem Interesse ist daher, ob die zu beobachtenden Entwicklungen eine europakompatible Ausgestaltung des Systems erzwingen und inwieweit dies dazu beitragen kann, ordnungspolitische Sünden der Vergangenheit aufzudecken und verkrustete Strukturen aufzubrechen. Neben der Darstellung der aktuellen Auswirkungen der Europäischen Integration in einzelnen Teilbereichen des deutschen Gesundheitswesens skizziert das folgende Kapitel mögliche europakompatible Lösungen sowie deren Auswirkungen auf die Wettbewerbsituation in den verschiedenen Bereichen. 


\section{Analyse der Auswirkungen}

\subsection{Vorbemerkungen}

In den folgenden Abschnitten stehen die Auswirkungen der Europäischen Integration auf wichtige Teilbereiche des Gesundheitswesens im Mittelpunkt des Interesses. Ausgangspunkt für diese Überlegungen waren die Urteile des EuGH über die Bedeutung der Waren- und Dienstleistungsfreiheit im Gesundheitswesen. Durch sie wurde deutlich, dass die nationalen Gesundheitssysteme keine europarechtsfreien Räume darstellen. Vielmehr erfordert die Integration der europäischen Märkte eine Öffnung der einzelstaatlichen Gesundheitsmärkte, da die vier Grundfreiheiten auch dort ihre Gültigkeit besitzen. Eine wesentliche Voraussetzung dafür, dass diese neu entstandenen Möglichkeiten tatsächlich Änderungen in den Systemen bringen, liegt jedoch in der Bereitschaft der Versicherten und Patienten, die gewonnenen Freiheiten auch zu nutzen. Die Auswirkungen einer wachsenden Patientenmobilität sind dabei insbesondere im Bereich der ambulanten ärztlichen Versorgung sowie auf dem Arzneimittelmarkt relevant.

Die Betrachtungen des vorangegangenen Kapitels haben jedoch auch gezeigt, dass die Einflussnahme der EU auf die Gesundheitsversorgung nicht erst mit den EuGHUrteilen begonnen hat und sich nicht allein auf die Durchsetzung der Grundfreiheiten beschränkt. Soweit hier aktuelle Entwicklungen bestehen, die isoliert in einzelnen Sektoren Veränderungen erwarten lassen, werden sie im Folgenden in den entsprechenden Abschnitten berücksichtigt. In allen untersuchten Bereichen spielen darüber hinaus internationale Vergleiche und Erfahrungen in anderen Mitgliedstaaten bei politischen Entscheidungen über die zukünftige Ausgestaltung des Gesundheitssystems eine wachsende Rolle. Diese Entwicklung könnte durch die Anwendung der OMK auf die Gesundheitsversorgung nochmals verstärkt werden. Tendenzen, die länderübergreifend in einer größeren Zahl anderer Mitgliedstaaten bei Reformen im Gesundheitswesen zu beobachten sind, sollen darum im Folgenden als zusätzlicher Hinweis für eine Abschätzung der zu erwartenden Entwicklung in Deutschland herangezogen werden.

In die Betrachtung mit einbezogen werden die ambulante vertragsärztliche Versorgung, der stationäre Sektor in seiner Trennung in Krankenhäuser sowie Vorsorge- und Rehabilitationseinrichtungen, der Arzneimittel- sowie der Apothekenmarkt. Im vierten Kapitel wird darauf eingegangen, ob sich durch die zu beobachtende Europäisierung der Gesundheitsmärkte ein Wettbewerb der verschiedenen Systeme ergibt und wie dieser zu bewerten ist.

\subsection{Ambulante und stationäre Versorgung}

\subsubsection{Patientenmobilität}

Die Rolle der Patienten ist bei der Frage nach den Auswirkungen der Europäischen Integration von großer Bedeutung, da der Umfang der durch die Integration verursachten Veränderungen in hohem Maße von der tatsächlichen Inanspruchnahme von Auslands- 
leistungen und damit von der grenzüberschreitenden Mobilität der Versicherten und Patienten abhängt. Eine wichtige Determinante möglicher Abwanderungen von GKVVersicherten ins Ausland sind dabei die Voraussetzungen für die dortige Leistungsinanspruchnahme. Wie in den Abschnitten 2.3.1.2 und 2.3.3.1 bereits angesprochen, bestehen für europäische Versicherte mit den Regelungen der Verordnung 1408/71/EWG (in Verbindung mit Verordnung 574/72/EWG) und der Inanspruchnahme auf Grundlage der Dienstleistungs- und Warenverkehrsfreiheit grundsätzlich zwei Verfahrenswege, um von ihrer Krankenkasse eine Kostenübernahme für im Ausland nachgefragte Gesundheitsleistungen zu erreichen.

Die beiden Möglichkeiten der ambulanten Leistungsinanspruchnahme im Ausland unterscheiden sich hinsichtlich der Auswirkungen auf die Patienten. Das europäische koordinierende Sozialrecht sieht in den Verordnungen 1408/71/EWG und 574/72/EWG ${ }^{210}$ - neben den Regelungen für sog. Grenzgänger - eine Möglichkeit der Kostenübernahme für Auslandsleistungen lediglich in Notfällen oder mit vorheriger Genehmigung des inländischen Kostenträgers vor. In diesen beiden Fällen besitzt der Versicherte einen Anspruch auf Leistungen des ausländischen Trägers, als ob er bei $\mathrm{ihm}$ versichert wäre. Die im Rahmen der Behandlung erbrachten Leistungen richten sich also nach dem Katalog des ausländischen Trägers, ${ }^{211}$ und der Versicherte hat ggf. die Zuzahlungen zu leisten, die im jeweiligen Land üblicherweise anfallen. Lediglich die Dauer der Leistungsgewährung orientiert sich an den Regelungen seines Heimatlandes. Die erforderliche Genehmigung für eine geplante Behandlung im Ausland muss die zuständige Krankenkasse erteilen, wenn die betreffende Behandlung zum inländischen Leistungskatalog gehört und der Versicherte in Anbetracht seines derzeitigen Gesundheitszustands und des voraussichtlichen Verlaufs der Krankheit diese Behandlung nicht in einem Zeitraum erhalten kann, der für diese Behandlung in seinem Wohnsitzstaat normalerweise erforderlich ist. ${ }^{212}$ Diese Regelungen finden in Bezug auf Krankenhausleistungen ihren Niederschlag explizit in $\S 13$ Abs. 5 SGB V. Auch nach der in Abschnitt 2.3.3.1 dargestellten Rechtsprechung des EuGH stellen sie für diese Leistungen die einzige Basis für eine grenzüberschreitende Inanspruchnahme mit Kostenerstattung der Krankenkassen dar. Wie der EuGH in seinen einschlägigen Urteilen mehrfach ausführte, gelten Waren- und Dienstleistungsfreiheit zwar grundsätzlich für alle Gesundheitsleistungen unabhängig davon, ob sie innerhalb oder außerhalb eines Krankenhauses erbracht werden. In Bezug auf Krankenhausleistungen erkannte der

\footnotetext{
${ }^{210}$ Diese Verordnungen gelten für alle Staaten des Europäischen Wirtschaftsraums (EWR) und für die Schweiz. Zum EWR gehören derzeit neben den 25 Mitgliedstaaten der EU auch die Lănder Liechtenstein, Island und Norwegen. Die Verordnungen werden aufgehoben von der Verordnung 883/2004/EG und der zugehörigen Durchfuhrungsverordnung, die ab dem Tag gelten, an dem die Durchfuhrungsverordnung in Kraft tritt. Derzeit erarbeitet die Kommission einen Vorschlag zu dieser Verordnung.

${ }^{211}$ In diesem Zusammenhang ist es wichtig zwischen der Behandlung, fur die die Genehmigung besteht, und den in der Behandlung enthaltenen Leistungen zu unterscheiden. Die Behandlung selbst muss im deutschen Leistungskatalog enthalten sein, damit eine Genehmigung zur Auslandbehandlung durch die Krankenkasse überhaupt möglich ist. Hinsichtlich der Fragen, welche Einzelleistungen während der Behandlung im Ausland erbracht und erstattet werden können, ist jedoch der ausländische Leistungskatalog relevant.

${ }_{212} \mathrm{Vgl}$. Art. 22, Abs. 2, Satz 2 Verordnung 1408/71/EWG.
} 
Gerichtshof jedoch an, dass eine Einschränkung der Freiheiten in Form einer vorherigen Genehmigung gerechtfertigt sei, um die Versorgung der Bevölkerung mit diesen Leistungen nicht zu gefährden. ${ }^{213}$

Da bei einer Behandlung mit vorheriger Genehmigung der Krankenkasse - wie bereits oben erwähnt - für die in der Behandlung enthaltenen Leistungen der ausländische Leistungskatalog gilt, hängt es von den Unterschieden zwischen ausländischem und deutschem Leistungskatalog ab, ob eine Inanspruchnahme ausländischer Behandlungen auf dieser Grundlage für deutsche GKV-Patienten attraktiv ist. Die Inanspruchnahme kann jedoch für die Versicherten im Vergleich zu einer entsprechenden inländischen Behandlung mit höheren Kosten einhergehen. Da die Übernahme der Kosten durch die inländische Krankenkasse nach den Bestimmungen des ausländischen Kostenträgers erfolgt, müssen die Patienten eventuell anfallende Zuzahlungen selbst tragen. Diese können u.U. wesentlich höher sein als die in Deutschland üblichen. So fallen beispielsweise in Frankreich bei einer Klinikbehandlung 20 Prozent der Kosten sowie eine Pauschale in Höhe von $14 € /$ Tag als Zuzahlung an. ${ }^{214}$ In Deutschland liegt dieser Selbstbehalt lediglich bei $10 € /$ Tag für die ersten 28 Tage. $^{215}$

Eine Änderung könnte sich hier durch die EuGH-Urteile in den Rechtssachen „Vanbraekel“ und „Watts" ergeben. ${ }^{216}$ Diese können dahingehend interpretiert werden, dass einem Versicherten, der sich auf Basis einer Genehmigung zu einer Behandlung ins Ausland begibt, zusätzlich zur Übernahme der im Ausland erstattungsfähigen Kosten eine weitergehende Erstattung zusteht, wenn die nach ausländischem Recht erstattungsfähigen Kosten zwar geringer sind als die Kosten einer entsprechenden Behandlung im Inland, der Versicherte im Ausland aber Zuzahlungen zu leisten hat. Diese wären vom inländischen Träger insoweit zu übernehmen, wie der Gesamtbetrag für die Auslandsbehandlung die Kosten für eine entsprechende Behandlung im Inland nicht übersteigt. Auf diese Weise könnten Behandlungen, die in ausländischen Mitgliedstaaten mit hohen Selbstbeteiligungen verbunden sind, im Rahmen einer Inanspruchnahme mit vorheriger Genehmigung für die Versicherten aus finanzieller Sicht an Attraktivität gewinnen. Voraussetzung dafür ist, dass die nach ausländischem Recht erstattungsfähigen Kosten geringer sind als die im Inland von den Kassen zu tragenden Behandlungskosten, da die Patienten nur dann eine (teilweise) Übernahme der im Ausland geleisteten Zuzahlungen erwarten könnten.

Im Fall der außerhalb von Krankenhäusern erbrachten Leistungen hat der EuGH anders als bei krankenhausbasierten Leistungen keinen Rechtfertigungsgrund für eine Beschränkung der Grundfreiheiten durch einen Genehmigungsvorbehalt gesehen. Für diese Leistungen existiert daher entsprechend der „Kohll/Decker"-Urteile die Möglichkeit zur grenzüberschreitenden Inanspruchnahme mit nachträglicher Kostenerstattung der zuständigen Krankenkasse, ohne dass eine vorherige Genehmigung notwendig ist. Durch das Urteil des EuGH in der Rechtssache „Inizan“ steht diese Möglich-

\footnotetext{
${ }^{213} \mathrm{Vgl}$. Europäischer Gerichtshof (2001a); Rn. 105 sowie die Ausführungen in Abschnitt 2.3.3.1.

${ }^{214} \mathrm{Vgl}$. Service Public Portail de l'Administration Française (2007).

${ }^{215} \mathrm{Vgl}$. § 61 SGB V.

${ }^{216}$ Siehe zu einer ausführlichen Darstellung der Urteile Abschnitt 2.3.3.1.
} 
keit gleichrangig neben den Regelungen der Verordnung 1408/71/EWG. ${ }^{217}$ Fragt der Patient entsprechend der „Kohll/Decker"-Rechtsprechung Behandlungen im Ausland nach, sieht sich die deutsche Krankenkasse allerdings lediglich zur Übernahme von Kosten in einer Höhe verpflichtet, wie sie bei einer vergleichbaren Behandlung im Inland entstanden wären bzw. - wenn diese geringer sind - bis zur Höhe der tatsächlich entstandenen Kosten, wobei die Kasse dazu verpflichtet ist, ausreichende Abschläge vom Erstattungsbetrag für Verwaltungskosten und fehlende Wirtschaftlichkeitsprüfungen vorzusehen sowie in Deutschland vorgesehene Zuzahlungen in Abzug zu bringen. ${ }^{218}$ Eine Kostenerstattung erfolgt grundsätzlich nur für solche Leistungen, die auch Bestandteil des deutschen Leistungskatalogs sind. Auch bei einer Inanspruchnahme entsprechend der „Kohll/Decker“-Urteile trägt der Patient ein Kostenrisiko, da er den Teil der im Ausland entstehenden Kosten selbst zahlen muss, der die in Deutschland geltenden Sätze für die entsprechende Behandlung übersteigt. Zudem darf der Patient im Rahmen einer Behandlung nur solche Leistungen wählen, die Bestandteil des deutschen Leistungskatalogs sind, da die Kassen die Kosten ansonsten nicht übernehmen.

Bei der Inanspruchnahme von nicht-krankenhausbasierten Leistungen ist deshalb zu prüfen, welches Verfahren für den Patienten die günstigere Option darstellt. Problematisch ist hierbei, dass den meisten Versicherten Informationen über die alternativen Möglichkeiten zur Inanspruchnahme von Auslandsleistungen fehlen, so dass sie nicht rational abwägen können, welche Option für sie Vorteile bietet. ${ }^{219}$ Die Informationen der Krankenkassen beschränken sich zumeist auf die Darstellung der Regelungen, die in Verordnung 1408/71/EWG festgeschrieben sind. Auf die Möglichkeit einer nachträglichen Kostenerstattung entsprechend der „Kohll/Decker"-Rechtsprechung und die damit verbundenen Regelungen wird kaum hingewiesen. Hier wäre eine offensivere Informationspolitik der Krankenkassen über die verschiedenen rechtlichen Möglichkeiten der Inanspruchnahme sowie ein Informationsangebot über die im Ausland entstehenden Behandlungskosten und Zuzahlungen sowie die entsprechenden deutschen Sätze und den deutschen Leistungskatalog wünschenswert, um die Patienten in die Lage zu versetzen, das für sie günstigere Verfahren zu wählen. Ein Anreiz dazu besteht für die Krankenkassen, wenn durch geringere Behandlungskosten im Ausland Kosteneinsparungen erzielt werden können. Darüber hinaus bietet die derzeitige Situation einzelnen Krankenkassen die Möglichkeit, sich durch ausführliche Informationen über die Kostenerstattungsregelungen bei Auslandsleistungen im Wettbewerb um neue Mitglieder von anderen Kassen abzuheben.

$\mathrm{Zu}$ beachten ist aber, dass die gesetzlichen Krankenkassen über die Erteilung einer Genehmigung nach der Verordnung 1408/71/EWG nicht frei entscheiden können. Entsprechend dieser Verordnung haben die Krankenkassen nur dann das Recht, die $\mathrm{Zu}$ stimmung zu einer Auslandsbehandlung zu verweigern, ,wenn die gleiche oder eine

${ }^{217}$ Vgl. Europäischer Gerichtshof (2003f), Rn. 21-22 und 51-60 sowie Jorens/Coucheir/Overmeiren (2005),S. 12.

${ }^{218}$ Vgl. $\$ 13$ Abs. 4 SGB V.

${ }^{219}$ Den Bedarf an Informationen über ausländische Gesundheitssysteme, aber auch das eigene System bei Patienten und Fachvertretern stellte auch der „Hochrangige Reflexionsprozess zur Patientenmobilität" der EU fest. Vgl. Europäische Kommission (2003f), S. 7f. 
für den Versicherten ebenso wirksame dem allgemein anerkannten Stand der medizinischen Erkenntnisse entsprechende Behandlung einer Krankheit rechtzeitig bei einem Vertragspartner $[\ldots]$ im Inland erlangt werden kann ${ }^{\prime 2220}$. Geht man davon aus, dass umgekehrt nur dann eine Genehmigung erteilt werden darf, wenn die genannten Anspruchsvoraussetzungen erfüllt sind, ${ }^{221}$ so setzen die betreffenden Regelungen einer Wanderung von GKV-Patienten ins europäische Ausland enge Grenzen und sind nicht geeignet, einen bedeutenden Einfluss auf die Ausgestaltung des deutschen Gesundheitswesens auszuüben. Die Möglichkeit einer Patientenwanderung in nennenswertem Umfang ist erst durch die EuGH-Rechtsprechung entstanden.

Ein Patient wird jedoch nur dann Gesundheitsleistungen grenzüberschreitend in Anspruch nehmen, wenn er davon ausgehen kann, dass die Qualität der Leistung im Ausland einen gewissen Standard erfüllt. Wie bereits in Abschnitt 2.3.3.1 erwähnt, lässt der EuGH mögliche Qualitätsunterschiede in der medizinischen Versorgung zwischen den Mitgliedstaaten nicht als Grund für eine Beschränkung der Dienstleistungsfreiheit gelten. Er entschied bereits im Urteil zur Rechtssache „Kohll“" im Jahre 1998, dass aufgrund zahlreicher Koordinierungs- und Harmonisierungsrichtlinien bezüglich der Zugangsbedingungen zum Beruf ausländische Leistungserbringer - namentlich Ärzte und Zahnärzte - als ebenso qualifiziert anzusehen sind wie im Inland tätige. ${ }^{222}$ Diese Auffassung wird vielfach kritisiert, da die entsprechenden EU-Vorschriften i.d.R. nicht die Ausübung des Berufes betreffen. ${ }^{223}$ Auf diese Weise erfolge zwar eine gewisse Sicherung der Strukturqualität, jedoch könne auf die Prozess- und Ergebnisqualität kein Einfluss genommen werden. Darum begrüßen zahlreiche Autoren die mit dem GMG neu geschaffene Möglichkeit, im Ausland tätige Leistungserbringer mittels Verträgen in das Sachleistungssystem der GKV einzubinden und auf diese Weise die inländischen Kontrollmechanismen zur Qualitätssicherung auf die grenzüberschreitende Inanspruchnahme auszuweiten. ${ }^{224}$ Eichenhofer (1999, S. 111) weist zudem darauf hin, dass durch Art. 93 der Verordnung 1408/71/EWG die Möglichkeit besteht, im Fall eines Kunstfehlers einen Ersatzanspruch an den ausländischen Träger durchzusetzen. Wird der Fehler im Inland auf Kosten der Krankenkasse behoben, geht dieser Ersatzanspruch kraft Gesetz auf sie über und sie kann diesen grenzüberschreitend geltend machen. Kötter (2003, S. 307) vertritt jedoch die Meinung, dass diese Regelung als Form der nachträglichen Qualitätssicherung zu kurz greift. Sie plädiert vielmehr dafür, auf Basis der Vertragsbestimmungen zum Gesundheitswesen (Art. 152 EGV), zum Verbraucherschutz (Art. 153 EGV) und zur Schaffung von Mindestvorschriften im Bereich der sozialen Sicherheit (Art. 137 Abs. 2b EGV) europaweite Mindeststandards zu schaffen. ${ }^{225}$ Zudem ist davon auszugehen, dass ein Haftungsanspruch bei Behandlungen mit vorheriger Genehmigung leichter durchsetzbar ist als bei selbst beschafften Leistungen, da unterschiedliche rechtliche Beziehungen bestehen. Im ersten Fall be-

\footnotetext{
${ }^{220} \mathrm{Vgl}$ § 13 Abs. 5 SGB V.

${ }^{221} \mathrm{Vgl}$. ähnlich Ribhegge (2005), S. 16.

${ }^{222} \mathrm{Vgl}$. Europäischer Gerichtshof (1998b), Rn. 48

${ }^{223}$ Vgl. z.B. Schulte (1999b), S. 275; Kötter (1999), S. 245; Fuchs (2004), S. 230.

${ }^{224}$ Vgl. z.B. Kingreen (2001), S. 3384; Becker (2003), S. 2275 f.

${ }^{225} \mathrm{Vgl.} \mathrm{Kötter} \mathrm{(2003),} \mathrm{S.} 307$.
} 
steht eine Verbindung der inländischen Krankenkasse zum ausländischen Träger, da dieser die entsprechenden Leistungen im Rahmen der Sachleistungsaushilfe erbringt und der inländischen Krankenkasse in Rechnung stellt. Im Fall selbst beschaffter Leistungen zahlt der Patient den ausländischen Leistungserbringer direkt und reicht die Rechnungen bei seiner Krankenkasse ein, die weder mit diesem Leistungserbringer noch mit einem ausländischen Träger in Kontakt treten muss.

Aufschluss darüber, welchen Risiken Versicherte bei der Inanspruchnahme ohne Genehmigung infolge der fehlenden grenzüberschreitenden Qualitätskontrollen ausgesetzt sind, können Erfahrungen mit der Notfallbehandlung im Ausland, wie beispielsweise der Behandlung von deutschen Skiunfallopfern in Österreich, geben. In diesen Fällen war eine vorherige Qualitätskontrolle ausländischer Ärzte und Krankenhäuser auch bisher nicht möglich. Bei dieser Diskussion sollte zudem Berücksichtigung finden, dass die Patienten selbst eine qualitativ hochwertige Versorgung suchen. Dieses Interesse des Patienten führt dazu, dass das Problem mangelnder Qualităt bei der Liberalisierung der europäischen Gesundheitsmärkte quantitativ eher von untergeordneter Bedeutung sein dürfte. Personen, die sich bewusst für eine Auslandsbehandlung entscheiden, werden der Qualität der Leistung eine große Bedeutung beimessen und sich im Vorfeld der Behandlung darüber informieren. ${ }^{226}$

Weitere Determinanten einer Nachfrage nach Gesundheitsleistungen im Ausland sind die Mobilität des Versicherten sowie eventuell zu erwartende Sprachprobleme. Diese beiden Aspekte sprechen dafür, dass eine grenzüberschreitende Leistungsinanspruchnahme vor allem in grenznahen Gebieten stattfinden wird. Zudem sind die Eigenschaften der medizinischen Leistungen von Bedeutung. Das Interesse der Versicherten wird sich vor allem auf Behandlungen richten, die möglichst wenig Arztbesuche umfassen und keine intensive Nachsorge erfordern. Bestehen im Inland fur Leistungen Qualitätsgarantien wie beim Zahnersatz in Deutschland, die bei einer Behandlung im Ausland entfallen, ist davon auszugehen, dass die Versicherten diese Leistung nur dann im Ausland nachfragen, wenn andere Gründe wie beispielsweise deutlich geringere Kosten dafür sprechen. Zusammenfassend hängt die Nachfrage nach Gesundheitsleistungen aus theoretischer Sicht von folgenden Bedingungen $a b:^{227}$

- rechtliche Möglichkeiten der Inanspruchnahme,

- Preisdifferenzen der Güter und Dienste,

- Qualitätsunterschiede bzw. Homogenität der Leistungen,

- Mobilität der Versicherten,

- Reputation des jeweiligen Gesundheitswesens bzw. der jeweiligen Leistungsanbieter,

- Eigenheiten der medizinischen Leistungen, wie einmal oder wiederkehrend, keine oder intensive Nachsorge, mit oder ohne Qualitätsgarantien,

${ }^{226}$ So auch Wille/Knerr (2000), S. 270.

${ }^{227} \mathrm{Vgl}$. Wille (1998), S. 30 
- Umfang und Art der Selbstbeteiligung im In- und Ausland,

- haftungsrechtliche Regelungen und Möglichkeiten der Rechtsdurchsetzung,

- Sprachbarrieren, die u.a. die Möglichkeiten zur Information und Beratung einschränken.

Die Europäische Kommission versuchte im Rahmen des sog. „Reflexionsprozesses auf hoher Ebene über die Patientenmobilität" Daten von den Mitgliedstaaten über das tatsächliche Ausmaß der grenzüberschreitenden Inanspruchnahme von Gesundheitsleistungen und die Aufteilung dieser Behandlungsfälle auf die beiden bestehenden Kostenübernahmeregelungen zu erhalten. ${ }^{228}$ Anhang B gibt in den Tabellen III bis V die dabei erhobenen Daten wieder. Diese weisen erhebliche Lücken auf, da verschiedene Länder keine Angaben machten, und sind schwer vergleichbar, da nicht überall (getrennte) Daten zu den beiden Inanspruchnahmemöglichkeiten vorlagen. Auch für Deutschland konnten im Rahmen des Reflexionsprozesses keine Zahlen gewonnen werden. Die Daten der aufgeführten Mitgliedstaaten lassen dennoch erkennen, dass sich das Ausmaß der Inanspruchnahme - sowohl was die Zahl der Behandlungsfälle als auch die damit verbundenen Zahlungen angeht - maximal im einstelligen Prozentbereich bewegt. Die Erhebung zeigt damit, dass die Patientenmobilität auch Jahre nach den „Kohll/Decker“-Urteilen des EuGH nur ein relativ geringes Ausmaß erreicht hat. Die Deutsche Verbindungsstelle Krankenversicherung Ausland (DVKA) ermittelte einen Betrag von rund 211 Mio. $€$, der im Jahre 2001 bundesweit für Aushilfeleistungen $^{229}$ aufgewandt wurde. ${ }^{230}$ Dies entspracht lediglich einem Anteil von 0,16 Prozent am Gesamtbudget der GKV in Deutschland im Jahre 2001 (ca. 130 Mrd. €). Eine im Jahr 2003 veröffent-lichte Mitgliederbefragung der Techniker Krankenkasse (TK) ergab, dass lediglich 7 Prozent der im Ausland in Anspruch genommenen medizinischen Leistungen zuvor geplant wurden. In allen anderen Fällen handelte es sich um Notfallbehandlungen. ${ }^{231}$

Da sich alle hier aufgeführten Daten auf die Jahre 1999 bis 2002 beziehen und erst die Urteile in den Rechtssachen „Smits und Peerbooms“ und „Müller-Fauré und van Riet“ in den Jahren 2001 bzw. 2003 die Bedeutung der EuGH-Rechtsprechung für alle europäischen Gesundheitssysteme deutlich machten - entsprechende Regelungen fanden vielfach erst danach Eingang in nationales $\operatorname{Recht}^{232}-$, könnten aktuellere Daten darüber Auskunft geben, inwieweit sich das Inanspruchnahmeverhalten der Versicherten seither geändert hat. Solche Daten sind derzeit jedoch nicht verfügbar. Es ist zudem

\footnotetext{
${ }^{228} \mathrm{Vgl}$. Anhänge zu Europäische Kommission (2003a).

${ }^{229}$ Nimmt ein EU-Bürger im Rahmen eines Notfalls oder mit vorheriger Genehmigung Leistungen in einem anderen Mitgliedstaat in Anspruch, so werden diese ihm vom ausländischen Träger als Sachleistungen gewährt. Diese Leistungen werden als „Aushilfeleistungen“ bezeichnet.

${ }^{230} \mathrm{Vgl}$. Sozialministerium Baden-Württemberg (2003), S. 9f.

${ }^{231}$ Vgl. Techniker Krankenkasse (TK) (2003), S. 14. Befragt wurden 6.000 Mitglieder der Techniker Krankenkasse mit einem Mindestalter von 18 Jahren, wobei über 2.100 Fragebögen ausgewertet werden konnten.

${ }^{232}$ In Deutschland wurde die Möglichkeit einer Kostenerstattung von im Ausland außerhalb von Krankenhäusem selbst beschafften Leistungen erst durch das GMG im Jahre 2004 im SGB V verankert.
} 
davon auszugehen, dass sich signifikante Veränderungen erst mittel- bis langfristig zeigen werden. Die Befragung der Techniker Krankenkasse zeigt aber auch, dass die TK-Versicherten einer Liberalisierung der Inanspruchnahme von Leistungen im europäischen Ausland überwiegend positiv gegenüber stehen. Nur 14 Prozent der Befragten bewerteten die Möglichkeit zur Wahl zwischen inländischen und ausländischen Leistungserbringern als negativ.

Ein großer Teil der beobachteten Behandlungsfälle konzentriert sich bisher in den sog. Euregios. Euregios sind eigenständige, transnationale Gebilde aus Grenzregionen benachbarter Staaten und können als grenzüberschreitende Regionen aufgefasst werden. Die EU fördert diese Zusammenschlüsse unter anderem durch die Gemeinschaftsinitiative zur transeuropäischen Zusammenarbeit (Interreg III 2000-2006). ${ }^{233}$ Die erste Euregio im deutsch-niederländischen Grenzgebiet entstand bereits 1958 im Raum von Enschede (Niederlande) und Gronau (Deutschland). Seither haben sich europaweit zahlreiche weitere grenzüberschreitende Kooperationen durch Euregios oder andere Formen entwickelt, die zum Teil auch bei der Gesundheitsversorgung der Bevölkerung zusammenarbeiten. In den vier Euregios im Grenzgebiet zwischen NordrheinWestfalen und den Niederlanden ${ }^{234}$ bestehen über 30 Projekte grenzüberschreitender Versorgung mit Gesundheitsleistungen. Beispielsweise haben in den Gebieten RheinWaal, Rhein-Maas-Nord und Maas-Rhein die AOK-Rheinland und die niederländische Kasse CZ eine gemeinsame Gesundheitskarte geschaffen (,Gesundheitscard International"), die die Versicherten dazu berechtigt, im Nachbarland allgemeine fachärztliche Behandlungen, Arzneimittel und stationäre Behandlungen in Form von Sachleistungen in Anspruch zu nehmen. Auf diese Weise wird der Weg der Patienten zum nächsten Arzt oder Krankenhaus verkürzt und eine bessere Auslastung freier Kapazitäten erreicht.

Darüber hinaus kann es für die Versicherten vorteilhaft sein, wenn die Mitgliedstaaten im Bereich der hochspezialisierten Einrichtungen miteinander kooperieren, beispielsweise in Form sog. Referenzzentren, wie sie der „Reflexionsprozess auf hoher Ebene über die Patientenmobilität" in seinem Abschlussbericht vorschlug. ${ }^{235}$ Auf diese Weise erfolgen eine Bündelung des Fachwissens und eine effizientere Allokation der vorhandenen Mittel, da speziell im Bereich der Erforschung und Behandlung seltener Krankheiten oftmals sehr hohe fixe Kosten entstehen.

Insgesamt eröffnet die Europäisierung des Gesundheitswesens den Versicherten und Patienten neue Möglichkeiten und bietet trotz der vorhandenen Risiken zahlreiche Chancen, qualitativ bessere oder kostengünstigere Leistungen im europäischen Ausland nachzufragen. Voraussetzung dafuir ist aber, dass sich die Patienten als Kunden begreifen und aktiv nach Informationen über entsprechende Behandlungs- und Kostenerstattungsmöglichkeiten suchen. Den Krankenkassen kommt dabei die Aufgabe zu, ihre Versicherten bei dieser Suche zu unterstützen. Letztlich können auch sie von

${ }^{233} \mathrm{Vgl}$. auch im Folgenden Greß/Axer/Wasem (2003), S. 85f.

${ }^{234}$ Dabei handelt es sich um die Euregios Enschede-Gronau, Rhein-Waal, Rhein-Maas-Nord und Maas-Rhein.

${ }^{235} \mathrm{Vgl}$. Europäische Kommission (2003f), S. 6. 
einer wachsenden grenzüberschreitenden Patientenmobilität profitieren, beispielsweise in Form von Kosteneinsparungen durch die Inanspruchnahme einzelner Leistungen wie Zahnersatz oder ambulante Kuren im Ausland.

Aus volkswirtschaftlicher Sicht sind die langfristigen Einflüsse der Nachfrageverschiebungen von großer Bedeutung, die in den verschiedenen Bereichen des Gesundheitswesens wirken und zu einer nachhaltigen Veränderung der Struktur der Gesundheitsversorgung in Deutschland beitragen können. Mögliche Auswirkungen dieser Einflüsse auf die im ambulanten Bereich tätigen Ärzte sowie die Krankenhäuser und Kureinrichtungen beschreiben die folgenden Abschnitte.

\subsubsection{Ambulante Versorgung}

Ambulante bzw. Vertragsärzte spielen im Gesundheitswesen eine wichtige Rolle als Anbieter von Gesundheitsleistungen. Bei der ökonomischen Betrachtung dieses Sektors gilt es zu beachten, dass sich die Märkte des Gesundheitswesens erheblich vom idealtypischen Markt der volkswirtschaftlichen Analyse unterscheiden. Da ein großer Teil der deutschen Bevölkerung im Rahmen der gesetzlichen Krankenversicherung gegen die finanziellen Risiken im Krankheitsfall abgesichert ist und ein fast eigenbeteiligungsfreier Zugang zu Gesundheitsleistungen besteht, reagiert die Nachfrage nach diesen Leistungen sehr preisunelastisch. Dadurch üben die Patienten als Nachfrager der Leistungen keine oder nur eine eingeschränkte Kontrolle im Hinblick auf die Effizienz der Leistungserbringung aus. Dagegen besitzen sie durchaus ein Interesse, die Qualität der Gesundheitsgüter zu überwachen. Doch auch hier ist die Kontrollmöglichkeit der Nachfrager sehr eingeschränkt, da sie selbst nicht über das nötige Wissen verfügen, eine Diagnose zu stellen, und die Qualität des ärztlichen Handelns nicht bewerten können. In der Regel hat der Arzt darum einen wesentlich größeren Entscheidungsspielraum als er Anbietern idealtypisch unter Wettbewerbsbedingungen zur Verfügung steht.

Wenn man nun die Anreizstruktur betrachtet, unter der Ärzte ihren Beruf ausüben, so ist davon auszugehen, dass ihre Zielsetzung sich nicht wesentlich von der anderer Akteure im Wirtschaftsgeschehen unterscheidet. Ein wichtiger Grund für die Berufswahl dürfte für sie die Erwartung eines überdurchschnittlichen Einkommens sein, und der Versuch, ein angemessenes Einkommen zu erzielen, dürfte ihr Angebotsverhalten prägen. Das ärztliche Standesethos kann hier zwar eine bindende Nebenbedingung darstellen, es ist jedoch davon auszugehen, dass die okkonomischen Rahmenbedingungen i.d.R. einen erheblichen Einfluss auf das Arztverhalten ausüben. Aus ökonomischer Sicht gilt es deshalb, die wirtschaftliche Anreizstruktur der Ärzte bei der Ausgestaltung der im vertragsärztlichen Bereich angewandten Regulierungen zu beachten, um das Ziel einer kostengünstigen und qualitativ hochwertigen Versorgung der Versicherten zu erreichen.

Dabei ist zunächst die Wahl des Vergütungssystems von Bedeutung. So bietet beispielsweise ein System, dass die Vergütung des Arztes an die Menge der erbrachten Leistungen knüpft, dem Arzt aus ökonomischer Sicht einen Anreiz, Leistungen zu 
verordnen, die medizinisch unnötig und unwirtschaftlich sind, um auf diese Weise sein Einkommen zu erhöhen. ${ }^{236}$ Eine solche angebotsinduzierte Nachfrage bietet einen Erklärungsansatz für die Beobachtung, dass die Erhöhung der Ärztedichte oftmals mit einem Anstieg der Inanspruchnahme der ärztlichen Leistungen pro Kopf einhergeht. ${ }^{237}$ Der Einfluss der ambulanten Ärzte durch ihr Überweisungs- und Verordnungsverhalten reicht dabei weit über den ambulanten Sektor hinaus. ${ }^{238}$ Die von den Vertragsärzten veranlassten Leistungen verursachten im Jahre $2001 \mathrm{mehr}$ als 70 Prozent aller GKVfinanzierten Leistungsausgaben, womit sie etwa viermal so hoch waren wie das von den Vertragsärzten selbst erbrachte Leistungsvolumen. Der Wunsch, die von den niedergelassenen Ärzten erbrachten und verordneten Leistungen zu begrenzen, hat daher zum Einsatz umfangreicher Regulierungsmaßnahmen im vertragsärztlichen Bereich geführt, die wettbewerbliche Elemente weitgehend ausklammerten, ohne letztlich dauerhaft die angestrebte Kontrolle der Kosten zu erreichen. Die Liberalisierung der Gesundheitsmärkte durch den europäischen Binnenmarkt wird darum vielfach begrüßt, da von ihr gerade auch in der ambulanten Versorgung eine Auflockerung verkrusteter Strukturen und ein stärkerer Wettbewerb zwischen den Leistungserbringern im Gesundheitswesen erwartet werden.

\subsubsection{Bedarfsplanung}

Die Bedarfsplanung stellt eine Begrenzung der Zahl der niedergelassenen Ärzte mit dem Ziel dar, einer ärztlichen „Überversorgung“ der Bevölkerung entgegenzuwirken. Sie ist eine Reaktion auf die Beobachtung, dass eine steigende Arzt- und Facharztdichte mit wachsenden Pro-Kopf-Ausgaben in der betreffenden Region einhergeht und soll dazu beitragen, die Kostensteigerung im Gesundheitswesen zu begrenzen. Beim derzeitigen Honorierungssystem kann sich eine steigende Zahl von Vertragsärzten zwar nicht (direkt) auf die Gesamthonorarsumme auswirken, weil diese nach oben begrenzt ist. Durch die große Bedeutung des Verordnungsverhaltens der Ärzte auf die Menge der veranlassten Leistungen würde sich aber ein expansiver Einfluss auf die Kosten für Arznei-, Heil- und Hilfsmittel ergeben.

Nachdem die Zulassung zur vertragsärztlichen Versorgung lange Zeit für alle Ärzte mit der erforderlichen Qualifikation ohne weitere Beschränkungen möglich war, schuf das „Gesetz zur Verbesserung der kassenärztlichen Bedarfsplanung“, das am 01.01.1987 in Kraft trat, die Möglichkeit zur Begrenzung der Zulassungen. ${ }^{239}$ Die Regelungen blieben jedoch weitgehend ohne Wirkung, da eine Steuerung der Zulassung

\footnotetext{
${ }^{236}$ Ein solches Verhalten des Arztes wird begünstigt durch die unvollkommenen Informationen des Patienten und eine quasi kostenlose Inanspruchnahme der Leistungen. Inwieweit dieser Anreiz besteht, hängt vom jeweiligen Vergütungssystem ab (vgl. dazu Abschnitt 3.2.2.2.1).

${ }^{237}$ Die These der angebotsinduzierten Nachfrage ist jedoch umstritten, da andere Erklärungsansătze existieren und somit aus dem empirisch relativ gut belegten Zusammenhang zwischen Ärztedichte und Pro-Kopf-Ausgaben für ärztliche Leistungen nicht zweifelsfrei auf eine Nachfrageinduzierung geschlossen werden kann. Vgl. Breyer/Zweifel/Kifmann (2005), S. 345ff; Sauerland (2002), S. 84ff.

${ }^{238} \mathrm{Vgl}$. Pfaff/Nagel (1995), S. 41.

${ }^{239} \mathrm{Vgl}$. im Folgenden Rosenbrock/Gerlinger (2004), S. 115.
} 
damit kaum möglich war: Erst nach einer Überschreitung der Bedarfskennzahlen um 50 Prozent konnte ein Planungsbereich geschlossen werden, wobei mindestens die Hälfte der Zulassungsbezirke geöffnet bleiben musste. Mit dem Gesetz zur Sicherung und Strukturverbesserung der GKV (Gesundheitsstrukturgesetz, GSG) traten im Jahre 1992 verschärfte Bestimmungen in Kraft, die mit geringen Veränderungen noch heute gelten.

Das SGB V sieht in den $\S \S 99$ bis 103 vor, dass Bedarfsplanungsrichtlinien zur Feststellung der Überversorgung erlassen werden. ${ }^{240}$ Anhand dieser Richtlinien werden arztgruppenspezifische Verhältniszahlen für den allgemeinen bedarfsgerechten Versorgungsgrad aus dem Verhältnis der Zahl der Einwohner zur Zahl der zugelassenen Vertragsärzte ermittelt. Einbezogen in die Bedarfsplanung wurden zunächst zwölf Arztgruppen. ${ }^{241}$ Stichtag für die Ermittlung der entsprechenden Verhältniszahlen war der 31.12.1990. Zum 1.1.1999 wurden darüber hinaus Anästhesisten und Psychotherapeuten in die Bedarfsplanung aufgenommen und entsprechende Verhältniszahlen ermittelt. Eine Überversorgung in einem Bezirk gilt als erreicht, wenn der allgemeine bedarfsgerechte Versorgungsgrad dort um 10 Prozent überschritten wird. Die Kassenärztlichen Vereinigungen erstellen auf Grundlage der Bedarfsplanungsrichtlinie im Einvernehmen mit den Landesverbänden der Krankenkassen und den Verbänden der Ersatzkassen den Bedarfsplan zur Sicherstellung der vertragsärztlichen Versorgung. Stellen die Landesausschüsse der Ärzte und Krankenkassen Überversorgung fest, müssen sie eine räumlich begrenzte und arztgruppenspezifische Zulassungssperre anordnen. Im entsprechenden Bezirk dürfen dann zeitlich begrenzt keine neuen Vertragsarztsitze ausgeschrieben werden, so dass eine Niederlassung als Vertragsarzt in einem solchen Bezirk nur durch die Übernahme einer frei werdenden Praxis oder im Rahmen einer Sonderbedarfszulassung ${ }^{242}$ möglich ist.

Abbildung 1 zeigt die Zahl der offenen Planungsbereiche in den Kassenärztlichen Vereinigungen zu Beginn des Jahres 2006. Während für Hausärzte ${ }^{243}$ die Niederlassung in 243 offenen Planungsbereichen (ca. 62 Prozent aller Planungsbereiche) möglich war, bestand für einzelne Facharztgruppen eine faktische Niederlassungssperre. ${ }^{244}$ Dies galt insbesondere für fachärztlich tätige Internisten, Chirurgen, Urologen, Hautärzte, Radiologen und Kinderärzte, für die weniger als 10 Prozent der Bezirke offen waren. Die sich daraus ergebenen Niederlassungsmöglichkeiten zu Beginn des Jahres 2006 für die verschiedenen Arztgruppen stellt Abbildung 2 dar.

\footnotetext{
${ }^{240} \mathrm{Vgl}$. im Folgenden Allgemeine Ortskrankenkasse (AOK) (2005b).

${ }^{241}$ Betroffen von der Bedarfsplanung waren zu diesem Zeitpunkt Hausärzte, Augenärzte, Nervenärzte, Orthopäden, HNO-Ärzte, Frauenärzte, Kinderärzte, Radiologen, Hautărzte, Urologen und Chirurgen.

${ }^{242} \mathrm{Vgl}$. Fußnote $54 \mathrm{im}$ Abschnitt 2.3.1.1.

${ }^{243} \mathrm{Zu}$ den Hausärzten zählen Allgemeinärzte, praktische Ärzte und hausärztlich tätige Internisten.

${ }^{244}$ Die Bedarfsplanung betrifft Arztgruppen, bei denen bundesweit mehr als 1.000 Ärzte an der vertragsärztlichen Versorgung teilnehmen. Vgl. § 101 Abs. 2 Satz 2 SGB V.
} 
Abbildung 1: Anzahl offener Planungsbereiche in den Kassenärztlichen Vereinigungen Anfang 2006

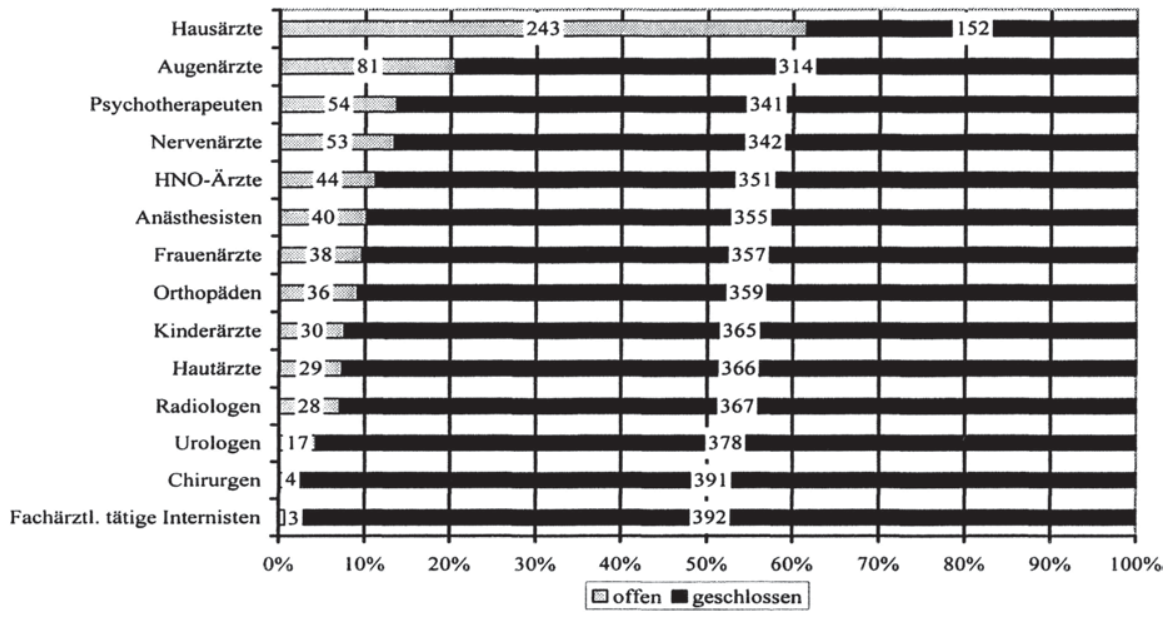

Quelle: Kassenärztliche Bundesvereinigung (KBV) (2007), Meldungen der Kassenärztlichen Vereinigungen

Abbildung 2: Niederlassungsmöglichkeiten nach Arztgruppen (Anfang 2006

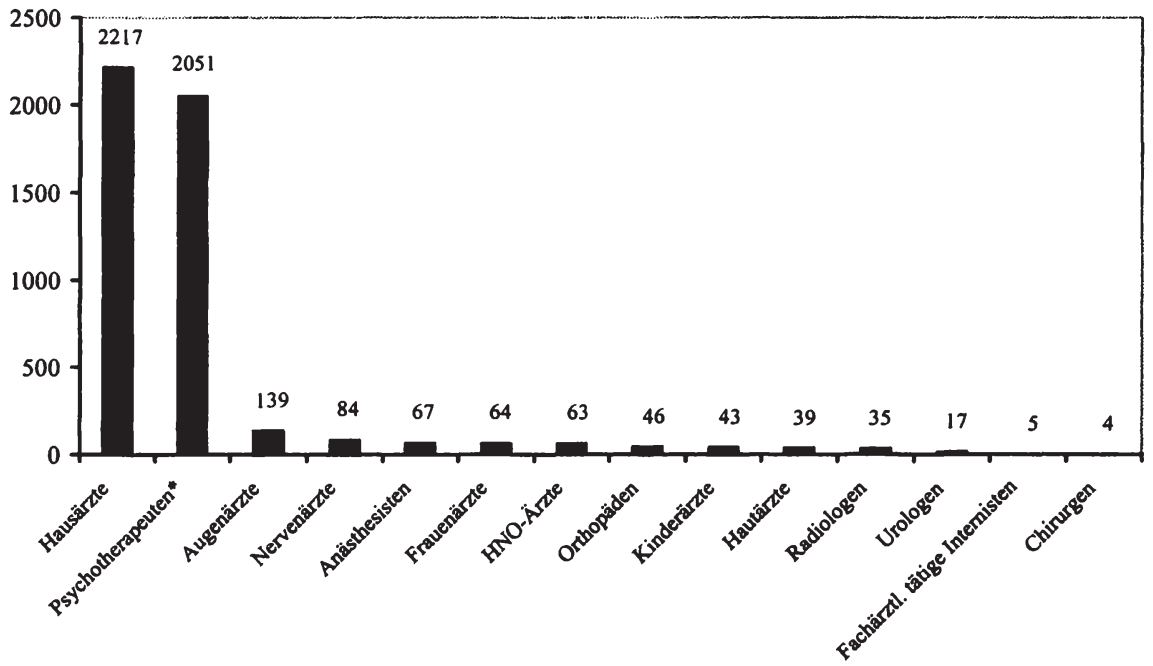

* einschließlich psychologischer Psychotherapeuten

Quelle: Kopetsch (2006), S. 398; eigene Darstellung 
Die Abbildung zeigt, dass für die meisten Fachärzte nur relativ wenige Möglichkeiten bestanden, als niedergelassener Vertragsarzt tätig zu werden. Für sie ergaben sich im gesamten Bundesgebiet je nach Arztgruppenzugehörigkeit zwischen 4 und $139 \mathrm{Nie}-$ derlassungsmöglichkeiten. Nur die Situation der Hausärzte und Psychotherapeuten ${ }^{245}$ stellte sich deutlich besser dar. Für sie bestand mit jeweils über 2.000 freien Arztsitzen eine deutlich bessere Chance, einen geeigneten Niederlassungsort zu finden. Änderungen dieser Gegebenheiten sind für die nähere Zukunft nicht zu erwarten.

Damit übt die Bedarfsplanung einen starken Einfluss auf die Niederlassungsmöglichkeiten der Fachärzte aus und schränkt somit deren Wahlfreiheit in Bezug auf den Ort ihrer Tätigkeit bedeutend ein. Damit entzieht sie diesen Teilarbeitsmarkt der Mediziner fast gänzlich einer wettbewerblichen Steuerung und stellt aus ordnungspolitischer Sicht einen starken planerischen Eingriff dar. Es ist daher nicht verwunderlich, dass die Grundfreiheiten und dabei insbesondere die Niederlassungs- sowie die Dienstleistungsfreiheit direkt und indirekt - über eine Erhöhung der Mobilität von Ärzten und Patienten - das Potential besitzen, in diesem Bereich Änderungen anzustoßen.

Die passive Dienstleistungsfreiheit entfaltet dabei durch Abwanderungen der Patienten ins Ausland ihre Wirkung. Da die Krankenkassen die Kosten ambulanter ärztlicher Behandlungen im Ausland übernehmen müssen, sind die deutschen gesetzlich Versicherten bei der Leistungsinanspruchnahme nicht mehr auf die inländischen Leistungserbringer beschränkt. Es steht ihnen frei, jeden Arzt innerhalb der EU bzw. des EWR aufzusuchen, der im jeweiligen Krankenversicherungssystem zur Versorgung der Patienten berechtigt ist, wodurch es theoretisch zu einer erheblichen Ausweitung der Kapazitäten im ambulanten Bereich kommt, die außerhalb der Steuerungsmöglichkeiten durch die inländische Bedarfsplanung liegt. Dies könnte dazu führen, dass die steigende Arztdichte insbesondere in grenznahen Gebieten mit einer Zunahme der Pro-KopfAusgaben einhergeht, zumal die im Ausland entstehenden Ausgaben bisher weder in die Gesamtvergütung der Vertragsärzte noch in die in Deutschland bestehenden Regelleistungsvolumina für Arznei- und Heilmittel einbezogen werden. ${ }^{246}$

Die Ausnahmen vom Territorialprinzip eröffnen zudem denjenigen Ärzten neue Optionen, die aufgrund der deutschen Regelungen keine Niederlassungsmöglichkeit im Inland erhalten. Sie können eine Praxis im grenznahen Ausland eröffnen, um insbesondere deutsche Patienten zu behandeln, was de facto eine Erhöhung der Arztdichte in dieser Region mit sich bringen würde. Zu beachten ist, dass bei dieser Konstellation die Sprachbarriere als wichtiges Mobilitätshemmnis wegfällt. Doch inwieweit besitzen deutsche Ärzte einen Anreiz, diese Strategie zu verfolgen? Neben den möglicherweise bestehenden Niederlassungsbeschränkungen im Ausland sind in dieser Hinsicht die

\footnotetext{
${ }^{245}$ Die zahlreichen Niederlassungsmöglichkeiten für Psychotherapeuten resultieren aus einer Mindestquote. Sowohl die Gruppe der ärztlichen als auch die der psychologischen Psychotherapeuten mulssen mindestens 40 Prozent aller Psychotherapeuten in einem Planungsbereich stellen (§101 Abs. 4 SGB V). Aufgrund dieser Regelung ergaben sich beispielsweise im Jahre 2005 für ärztliche Psychotherapeuten 1.814 Niederlassungsmöglichkeiten, für psychologische Psychotherapeuten dagegen nur 237 . Vgl. Kopetsch (2006), S. 398; eigene Berechnungen.

${ }^{246} \mathrm{Vgl}$. dazu ausführlicher die Abschnitte 3.2.2.2 und 3.2.2.3.
} 
zukünftige Entwicklung der Niederlassungsmöglichkeiten im ambulanten Bereich sowie die Beschäftigungsmöglichkeiten außerhalb dieses Sektors von wesentlicher Bedeutung.

Lediglich für die Gruppe der Hausärzte zeigt sich - insbesondere in den fünf neuen Bundesländern - ein etwas anderes Bild. Hier existiert bereits heute eine große Zahl freier Arztsitze. Zudem lag hier der Anteil der Hausärzte, die 60 Jahre oder älter waren, im Jahre 2002 zwischen 21,3 und 27,9 Prozent und damit jeweils deutlich über dem Bundesdurchschnitt von 15,6 Prozent. Die Prognose des Wissenschaftlichen Instituts der AOK (WIdO) ergab, dass in den Jahren 2002 bis 2010 insgesamt nochmals 2.684 hausärztliche Vertragsarztsitze in diesen Ländern frei werden.

Abbildung 3: Entwicklung der Altersstruktur der Vertragsärzte

Zahl der Ärzte je Jahrgang

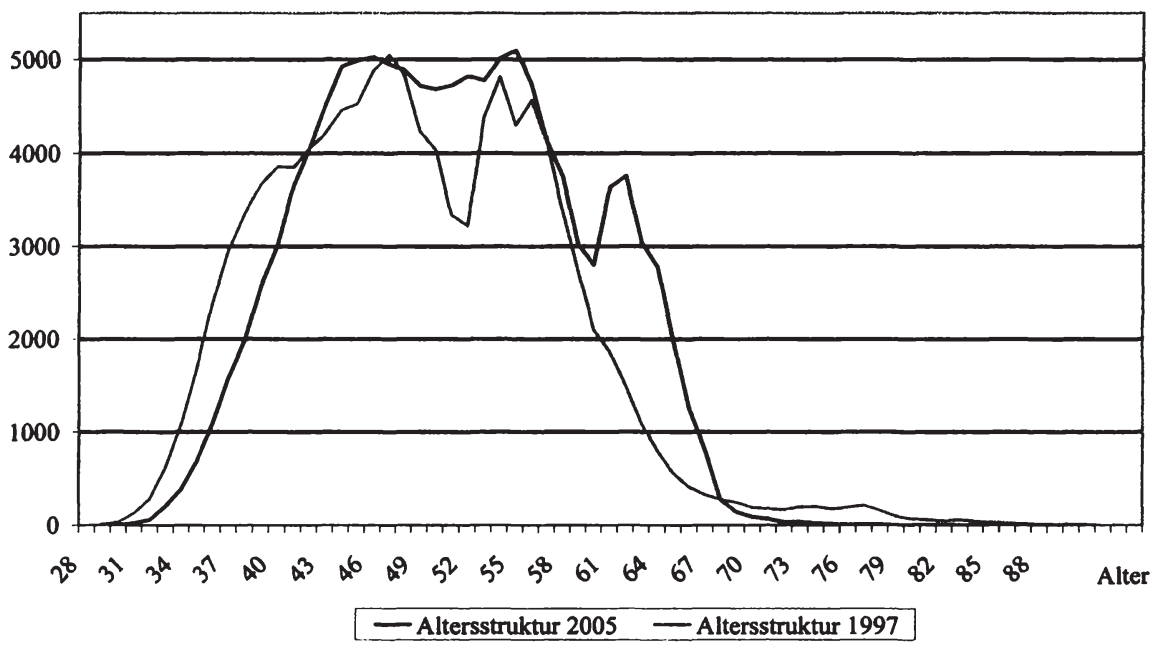

Quelle: Kassenärztliche Bundesvereinigung (KBV) (2007); eigene Darstellung

Abbildung 3 vergleicht die Altersstruktur der Vertragsärzte zum 31.12.1997 mit der Struktur des Jahres 2005. Es zeigt sich eine Rechtsverschiebung der Kurve, d.h. das Durchschnittsalter der Vertragsärzte ist im betrachteten Zeitraum angestiegen. Aus den zugrunde liegenden Daten ${ }^{247}$ lässt sich errechnen, dass der Anteil der Ärzte, die $60 \mathrm{Jah}-$ re oder älter sind, von 10,3 auf 17,5 Prozent stieg, während der Anteil der jüngeren Ärzte unter 40 Jahren von 18,2 auf 7,2 Prozent deutlich sank. Das bedeutet, dass in den nächsten zehn Jahren ein relativ großer Teil der Ärzte die Altersgrenze von 68 Jahren erreichen und damit aus der vertragsärztlichen Versorgung ausscheiden

${ }^{247}$ Vgl. Kassenärztliche Bundesvereinigung (KBV) (1998), Tabelle A 25 und Kassenärztliche Bundesvereinigung (KBV) (2007), S. 28. 
wird. ${ }^{248} \mathrm{Da}$ eine große Zahl der Planungsbereiche für die Facharztgruppen von Überversorgung geprägt ist, ${ }^{249}$ ergibt sich daraus jedoch kein bedeutender Ersatzbedarf.

Lediglich für die Gruppe der Hausärzte zeigt sich - insbesondere in den fünf neuen Bundesländern - ein etwas anderes Bild. Hier existiert bereits heute eine große Zahl freier Arztsitze. Zudem lag hier der Anteil der Hausärzte, die 60 Jahre oder älter waren, im Jahre 2002 zwischen 21,3 und 27,9 Prozent und damit jeweils deutlich über dem Bundesdurchschnitt von 15,6 Prozent. ${ }^{250}$ Die Prognose des Wissenschaftlichen Instituts der AOK (WIdO) ergab, dass in den Jahren 2002 bis 2010 insgesamt nochmals 2.684 hausärztliche Vertragsarztsitze in diesen Ländern frei werden. ${ }^{251}$

Im Hinblick auf die Beschäftigungsmöglichkeiten lässt sich damit zusammenfassen, dass im Bereich der niedergelassenen Fachärzte der Bedarf an Ärzten heute und auch in der näheren Zukunft weitgehend gedeckt ist, wodurch keine signifikant steigende Zahl von Neuzulassungen zu erwarten ist. Im hausärztlichen Bereich ergeben sich dagegen - insbesondere in den fünf neuen Bundesländern - deutlich bessere Beschäftigungsmöglichkeiten. Allerdings zeigt die Entwicklung der vergangenen Jahre eine stetige Abnahme der zugelassenen Hausärzte, was darauf hindeutet, dass diese Tätigkeit für junge Ärzte unattraktiv ist und aus ihrer Sicht keine Alternative zur fachärztlichen Tätigkeit oder anderen Beschäftigungsmöglichkeiten darstellt.

Aufgrund fehlender Niederlassungsmöglichkeiten in Deutschland kann eine Niederlassung im grenznahen Ausland, um dort deutsche Patienten behandeln zu können, die sich auf Basis der „Kohl-/Decker"-Rechtsprechung ins Ausland begeben, für Fachärzte in Einzelfällen durchaus eine attraktive Option darstellen. Diese Möglichkeit kann zudem für Ärzte interessant sein, die aufgrund der Altersbegrenzung ihre Kassenzulassung verloren haben, aber mit der Eröffnung einer Praxis im Nachbarland ihre bisherigen Patienten weiter behandeln können. Es ist jedoch davon auszugehen, dass dieser Weg nur von einer überschaubaren Zahl von Medizinern gewählt werden wird, da verschiedene Faktoren gegen eine hohe Mobilität der deutschen Patienten sprechen. Solange für die Abrechnung der in Deutschland erbrachten Leistungen das Sachleistungsprinzip gilt, bleibt die Kostenerstattung für die deutschen Patienten ein Hindernis bei der grenzüberschreitenden Nachfrage, zumal sie u.U. mit einem Eigenanteil an den

\footnotetext{
${ }^{248}$ Die Zulassung als Vertragsarzt endet i.d.R. am Ende des Kalendervierteljahres, in dem der Vertragsarzt 68 Jahre alt wird - vorausgesetzt, der Arzt war bis zu diesem Zeitpunkt mindestens 20 Jahre zugelassen. Durch das Gesetz zur Änderung des Vertragsarztrechts und anderer Gesetze (Vertragsarztrechtsănderungsgesetz - VÄndG) vom 22.12.2006 hat sich hier eine Verănderung ergeben. Demnach greift die Altersgrenze von 68 Jahren nicht, wenn vom Landesausschuss der Ärzte und Krankenkassen festgestellt wurde, dass in einem bestimmten Gebiet des Zulassungsbezirks ärztliche Unterversorgung besteht oder unmittelbar droht. Vgl. § 95 Abs. 7 SGB V.

${ }^{249} \mathrm{Vgl}$. Klose/Uhlemann/Gutschmidt (2003). Die Studie befasst sich mit der Situation in den östlichen Bundesländern und in Bayern im Jahre 2002. Es ist davon auszugehen, dass sich die Situation in den Planungsbezirken der westlichen KVen ähnlich darstellt.

${ }^{250} \mathrm{Vgl}$. Klose/Uhlemann/Gutschmidt (2003), Abbildung 7, Seite 13.

251 Die Berechnung erfolgt unter der Annahme, dass alle Vertragsärzte mit Vollendung des 68. Lebensjahres aus der vertragsärztlichen Versorgung ausscheiden. Vgl. dazu auch die Anmerkung in Fn. 247.
} 
Kosten, höheren Zuzahlungen und rechtlichen Unsicherheiten rechnen müssen. Aufgrund der hohen durchschnittlichen Arztdichte in Deutschland ist zudem davon auszugehen, dass in den meisten Gebieten keine Mangelsituation besteht, die eine grenzüberschreitende Nachfrage erforderlich machen könnte. Darüber hinaus deutet die weit unterdurchschnittliche Arbeitslosenquote bei Medizinern darauf hin, ${ }^{252}$ dass trotz einer geringen Zahl von Niederlassungsmöglichkeiten im fachärztlichen Bereich neben der hausärztlichen Tätigkeit weitere Beschäftigungsmöglichkeiten in anderen Feldern bestehen. Dafür spricht auch, dass viele Personalverwaltungen in Krankenhäusern einen Mangel an ärztlichem Personal beklagen. ${ }^{253}$ Offenbar entscheiden sich immer mehr Mediziner gegen die Tätigkeit am Patienten und wählen Beschäftigungsalternativen beispielsweise in der Pharmaindustrie oder im Medizinjournalismus. ${ }^{254}$

So hat die Rechtsprechung des EuGH mit den Urteilen zur passiven Dienstleistungsfreiheit eine Möglichkeit geschaffen, die Regelungen der Bedarfsplanung zu umgehen. Auch wenn aufgrund der zu erwartenden geringen Zahl von Niederlassungen deutscher Ärzte im Ausland hiervon keine gravierenden Auswirkungen auf das Gesamtsystem ausgehen werden, hat die Rechtsprechung dazu beigetragen, die starren deutschen Regulierungen in diesem Bereich zu durchbrechen, was zu einem stärkeren Wettbewerb zwischen den Leistungserbringern vor allem in Grenzgebieten beitragen kann.

Doch nicht nur die passive, auch die aktive Dienstleistungsfreiheit besitzt grundsätzlich das Potential, im bestehenden System Veränderungen anzustoßen. Sie ermöglicht Ärzten, die in einem anderen EU-Mitgliedstaat zugelassen sind, eine vorübergehende Tätigkeit in Deutschland aufzunehmen. Folgt man der Argumentation von Jorens/Coucheir/Overmeiren (2005), die u.a. auf der „Kohll/Decker“-Rechtsprechung des EuGH basiert, müssten diese Ärzte aus rechtlicher Sicht eine gleichberechtigte Möglichkeit erhalten, am Vertragssystem des Aufnahmemitgliedstaates teilzunehmen. ${ }^{25 s}$ Doch diese Option allein reicht nach Ansicht der Autoren nicht aus, um eine Beschränkung der Dienstleistungsfreiheit zu verhindern. Erst wenn die Krankenkassen die Kosten der von diesen Ärzten erbrachten Leistungen außerhalb des Vertragssystems durch eine nachträgliche Kostenerstattung übernähmen, besäßen diese Ärzte tatsächlich die Möglichkeit, ihre Freiheit zur vorübergehenden Erbringung von Dienstleistungen zu nutzen. Würde eine größere Zahl von ausländischen Ärzten diese Option wählen, liefe die nationale Bedarfsplanung notgedrungen ins Leere. Voraussetzung wäre allerdings, dass die Patienten diese Leistungserbringer tatsächlich in Anspruch

${ }^{252}$ Im Jahre 2003 betrug die jahresdurchschnittliche Arbeitslosenquote für das gesamte Bundesgebiet 10,5 Prozent; die entsprechende Quote für Akademiker wird auf ca. 4 Prozent geschätzt. Nochmals günstiger stellte sich die Situation der Humanmediziner dar. Die hier errechnete Quote von unter 2 Prozent furr das Jahre 2003 deutet auf eine weitgehende Vollbeschäftigung mit lediglich friktioneller Arbeitslosigkeit hin. Vgl. Bundesagentur furr Arbeit (BfA) / Zentralstelle für Arbeitsvermittlung (ZAV) (2004a), S. $6 f$ und 55. Einen leichten Anstieg der Quote (auf 2,1 Prozent) gab es im Jahre 2004. Vgl. Bundesagentur für Arbeit (BfA) / Zentralstelle für Arbeitsvermittlung (ZAV) (2004b), S.

18.

${ }^{253} \mathrm{Vgl}$. Fiehn/Schinnenburg (2004), S. A 626.

${ }^{254} \mathrm{Vgl}$. Kopetsch (2004), S. 316.

${ }^{255}$ So auch Jäger-Lindemann (2004), S. 83. 
nehmen, zumal auch hier die Kostenerstattung ein Hindernis darstellen könnte. In diesem Kontext wäre die Umsetzung des Vorschlags der Kommission für eine Richtlinie über Dienstleistungen im Binnenmarkt von großer Bedeutung gewesen. Sie hätte es ausländischen Leistungserbringern ermöglicht, deutschen Versicherten im Inland Behandlungen zu den Konditionen ihres Heimatlandes - also ggf. auch zu geringeren Preisen - anzubieten. Die Patienten hätten sich dann für eine Behandlung bei einem ausländischen Arzt nicht mehr ins Ausland begeben müssen. Da bereits heute Behandlungen mit hohen Zuzahlungen im Inland (z.B. Zahnersatzbehandlungen) in nennenswertem Umfang im Ausland nachgefragt werden, ist davon auszugehen, dass ein Angebot dieser Leistungen in Deutschland für deutsche Versicherte grundsätzlich interessant gewesen wäre. Durch die Herausnahme der Gesundheitsdienstleistungen aus dem Kommissionsvorschlag steht eine solche Regelung derzeit jedoch nicht mehr zur Diskussion.

Auswirkungen, die die Bedarfsplanung in Fragen stellen könnten, sind aber auch auf anderem Wege möglich. Wie im Abschnitt 2.3.1.1 ausgeführt, wird in der rechtswissenschaftlichen Literatur die Auffassung vertreten, dass die Bedarfsplanung dann gegen die europäische Niederlassungsfreiheit verstößt, wenn sie dazu führt, dass einem Arzt mit einem in einem anderen EU-Mitgliedstaat erworbenen Diplom die Niederlassung als Vertragsarzt in Deutschland verwehrt bleibt. Daraus ergäbe sich, dass diese Ärzte einen Niederlassungsort in Deutschland auswählen könnten und zudem das Recht hätten, an der Versorgung der GKV-Versicherten teilzunehmen. Inwieweit sich daraus Konsequenzen für die Arztzahlen ergeben, hängt davon ab, ob diese Option für eine ausreichend große Zahl von Ärzten interessant ist.

Besonderes Interesse verdienen dabei zwei Aspekte. Zum einen sind mit der EUOsterweiterung zahlreiche Ärzte EU-Bürger geworden, deren berufliche Situation in ihrem Heimatland sehr unbefriedigend ist und die durch eine Niederlassung als Facharzt in Deutschland auch finanziell deutlich profitieren könnten. ${ }^{256}$ Für Ärzte, die ihre Aus- oder Weiterbildung vor dem Beitritt ihres Staates zur EU begonnen haben, kann es dabei zwar zu Problemen bei der Anerkennung ihres Diploms in anderen Mitgliedstaaten kommen. Begann die Ausbildung jedoch nach dem 1. Mai 2004 - dem Datum des Beitritts dieser Länder zur EU -, so gilt auch für diese Diplome die automatische Anerkennung entsprechend der Richtlinie 93/16/EWG. Zum anderen sind die Grundfreiheiten immer dann anwendbar, wenn ein Arzt sein Diplom in einem anderen EUStaat als dem Land seiner Staatsangehörigkeit erworben hat, so dass aufgrund der Niederlassungsfreiheit auch einem deutschen Arzt mit einem Diplom aus einem anderen Mitgliedstaat eine Zulassung als Vertragsarzt nicht verweigert werden könnte. In diesem Zusammenhang ist von besonderer Relevanz, dass der EuGH im Juli 2005 die bis dahin geltenden Zulassungsvoraussetzungen für das Medizinstudium in Österreich als europarechtswidrig ansah. In Österreich stand der Zugang zum Medizinstudium allen Bewerbern mit österreichischem Reifezeugnis (Matura) offen, während Bewerber mit entsprechenden ausländischen Hochschulreifezeugnissen einen Studienplatz im Land ihres Schulabschlusses nachweisen mussten. Durch das Urteil des Gerichtshofes konn-

${ }^{256}$ Vgl. zur Situation der Ärzte in Polen Merten (2004a). 
te dieses Verfahren ab dem Jahre 2006 nicht mehr angewandt werden. Relevant ist diese Entwicklung in Bezug auf die hier untersuchte Fragestellung, da deutsche Medizinstudenten mit einem österreichischen Studienabschluss das Recht zur freien Niederlassung als Vertragsarzt in Deutschland erhalten würden, wenn die in Deutschland praktizierte Bedarfsplanung tatsächlich gegen die europäische Niederlassung verstößt. Wie groß die Zahl deutscher Medizinstudenten in Österreich langfristig tatsächlich sein wird, hängt u.a. davon ab, welche alternativen Zulassungsbeschränkungen die dortigen Universitäten ergreifen werden. Im Studienjahr 2006 kam es zu einem spürbaren Zustrom deutscher Studienanfänger an den österreichischen Fakultäten für Medizin. ${ }^{257}$ In Reaktion auf das EuGH-Urteil wurden in Österreich Quoten für den Zugang zum Medizinstudium eingeführt, wonach 75 Prozent der Studienplätze für Inhaber österreichischer Reifezeugnisse vorgesehen sind und lediglich 20 Prozent an Studienbewerber aus anderen EU-Staaten und 5 Prozent an Bewerber aus Nicht-EU-Staaten vergeben werden. Nun ist zwar damit zu rechnen, dass diese Regelungen ebenfalls gegen europäisches Recht verstoßen, allerdings könnten bei einer entsprechenden Klage eines abgewiesenen Studenten oder der EU-Kommission mehrere Jahre bis zur Entscheidung des EuGH vergehen.

Damit lässt sich zusammenfassend ein wachsender Einfluss der Grundfreiheiten auf die deutsche Bedarfsplanung feststellen, der insbesondere bei einem möglichen Verstoß gegen die Niederlassungsfreiheit spürbare Konsequenzen haben könnte. Sollte sich aufgrund der dargestellten Entwicklungen tatsächlich eine signifikante Zunahme der freien Niederlassungen von Ärzten mit ausländischen Diplomen in Deutschland ergeben, würde die Abschottung dieses Arbeitsmarktes partiell aufgebrochen, was zu einem stärkeren Wettbewerb der Leistungserbringer führen würde. Zudem stellt sich dann die Frage nach einer Gleichbehandlung von In- und Ausländern. Zwar kann diese durch die Grundfreiheiten nicht erzwungen werden, da eine Schlechterstellung von Inländern ohne grenzüberschreitenden Bezug nicht nach europäischen Rechts zu bewerten ist, dennoch bietet eine solche Inländerdiskriminierung eine Konfliktpotential, das durch die Einführung von weniger restriktiven Maßnahmen entschärft werden kann. ${ }^{258}$

Auch die Dienstleistungsfreiheit führt, selbst für den realistischen Fall einer zumindest in der kurzen Frist sehr geringen Mobilität von Ärzten und Patienten, zu einer Durchbrechung des kollektivvertraglich gestalteten Systems und liefert damit einen Anstoß, über alternative Steuerungsformen nachzudenken, die mehr Freiraum für wettbewerbliche Elemente lassen.

Diese Überlegungen haben Konsequenzen für weitere wichtige Steuerungselemente im Gesundheitswesen, da mit einem möglichen Wegfall der Bedarfsplanung ein Instrument zur Eindämmung der insgesamt erbrachten Leistungsmenge verloren ginge. Damit wäre es umso wichtiger, Vergütungsstrukturen zu entwickeln, die den Ärzten Anreize zu einer wirtschaftlichen Behandlung der Patienten bieten - insbesondere auch in

\footnotetext{
${ }^{257} \mathrm{Vgl}$. Spiegel Online (2006).

${ }^{258}$ Pitschas weist darauf hin, dass inländische Bewerber eine ausschließliche Gültigkeit von Zulassungsbeschränkungen für sie als eine willkürliche Ungleichbehandlung betrachten könnten, die mit dem Art. 3 Abs. 1 Grundgesetz (GG) nicht vereinbar sei. Vgl. Pitschas (1994), S. 109.
} 
Bezug auf die von ihnen verordneten Leistungen. Im Hinblick auf die Öffnung der Grenzen für Gesundheitsleistungen ist es dabei wünschenswert, dass diese Vergütungssysteme auch grenzüberschreitend angewandt werden können, ohne dass die Einbeziehung im Ausland niedergelassener Ärzte systemfremder Verfahren bedarf.

\subsubsection{Vertragsärztliche Vergütung}

\subsection{Varianten der vertragsärztlichen Vergütung}

Durch die Wahl des Vergütungssystems kann das Angebotsverhalten der ambulanten Ärzte wesentlich beeinflusst werden. Pfaff/Nagel (1995) formulieren in Anlehnung an das Jahresgutachten 1988 des SVRKAiG die Anforderungen an ein rationales Vergütungssystem folgendermaßen: "Es soll u.a. keine Anreize zu medizinisch ungerechtfertigten Leistungsmengenausweitungen geben, das medizinisch Erforderliche aber in guter Qualität sicherstellen, überflüssige Doppelleistungen vermeiden und zu einer funktionsgerechten Arbeitsteilung innerhalb der ambulanten Versorgung und zwischen dem ambulanten und stationären Sektor führen. Gleichzeitig soll die notwendige Fachkunde gesichert, das Aus- und Weiterbildungsniveau gefördert und ein möglichst hoher technischer Standard gewährleistet werden. Schließlich sollte eine Patientenselektion vermieden werden, und es sollten einfache Prüfmöglichkeiten und ein hohes $\mathrm{Ma} ß$ an Flexibilität bestehen." 259

Eine in der aktuellen Diskussion um die Reform des bestehenden Systems immer wieder erhobene Forderung stellt die Orientierung der Honorierung der Ärzte am Ergebnis ihrer Arbeit - dem Outcome - dar. Eine solche Vergütung hat den Vorteil, dass sie dem Arzt einen Anreiz bietet, die Behandlung zu wählen, die den Patienten rasch gesunden lässt. Allerdings stößt man bei der Realisierung einer solchen Vergütung im modernen Gesundheitssystem auf verschiedene Hindernisse. Obwohl die Evaluation von Gesundheitsleistungen einen immer breiteren Raum in der Gesundheitsökonomie einnimmt, ist die Messung des Ergebnisses der ärztlichen Tätigkeit immer noch mit gravierenden Problemen verbunden: ${ }^{260}$ Zum einen ist der Heilerfolg schwer messbar, was sich bereits darin zeigt, dass eine praktikable Definition von Gesundheit bisher nicht existiert. Zum anderen hängt der Behandlungserfolg nicht allein von der ärztlichen Leistung, sondern auch von zahlreichen anderen Faktoren ab, deren Einfluss jedoch wiederum nicht erfassbar ist, so dass der Beitrag des Arztes zur Genesung des Patienten unklar bleibt. Und zuletzt ist bei vielen Krankheiten eine Gesundung nur langfristig oder nicht vollständig erreichbar. Hier kann der Arzt oftmals nur die gesundheitlichen Einschränkungen lindern. Aus diesen Gründen ist eine erfolgsorientierte Vergütung aus heutiger Sicht nicht praktikabel.

${ }^{259}$ Vgl. SVRKAiG (1988), Ziffer 148, S. 54, zitiert nach Pfaff/Nagel (1995) S. 41.

${ }^{260} \mathrm{Vgl}$. Schulenburg (1992), S. 119. 
Alternativ stehen verschiedene Systeme zur Auswahl, deren Grundlage messbare Kriterien bilden, die das Leistungsgeschehen erfassen sollen. Als Bemessungskriterien sind dabei beispielsweise denkbar: ${ }^{261}$

- die Menge der erbrachten Einzelleistungen (Einzelleistungsvergütung),

- die Zahl der "potentiellen" Patienten, die sich zu Beginn der Periode bei einem Arzt ihrer Wahl einschreiben (Kopfpauschale) bzw. die Zahl der durchgefuihrten Behandlungen (Fallpauschale) oder

- die Abrechnungsperiode (Fixum).

Je nach Wahl des Bemessungskriteriums erhält der Arzt durch die Vergütung unterschiedliche Anreize hinsichtlich der Wirtschaftlichkeit und der Qualităt der gewählten Behandlung. Eine Honorierung mittels Einzelleistungsvergütung setzt die vorherige Bewertung der einzelnen Leistungen voraus. ${ }^{262}$ Wird die Einzelleistungsvergütung in Verbindung mit einer zentralen Honorarfestsetzung angewandt, so liegt diesem Verfahren eine Gebührenordnung zugrunde, in der alle abrechenbaren Leistungen mit einem Geldbetrag oder einem Punktwert aufgeführt sind. Durch die damit vorgenommene relative Bewertung der Leistungen ist es theoretisch möglich, die Erbringung von besonders wünschenswerten Leistungen - z.B. zuwendungsorientiertere Behandlungen im Sinne einer "sprechenden" Medizin - zu fördern.

Bei der Produktion der Einzelleistungen besteht ein Anreiz zur effizienten Erstellung, da dadurch bei gegebenen Gebührensätzen der Gewinn pro erbrachter Leistung maximiert wird. Allerdings besitzt der Arzt kein Interesse an der Wahl einer kostenminimalen Kombination von Einzelleistungen. Solange die gewählten Einzelleistungen einen positiven Deckungsbeitrag aufweisen ist es für ihn bis zum Erreichen seiner Kapazitätsgrenze vielmehr rational, diejenige Behandlung zu wählen, die bei gleichem $\mathrm{Be}-$ handlungserfolg mehr Einzelleistungen erfordert bzw. zusätzliche Einzelleistungen zu erbringen, die für die Genesung des Patienten nicht notwendig sind.

Die Einzelleistungsvergütung führt damit zu einer Ausdehnung der ärztlichen Leistungen, die sich in der Praxis in verschiedenen Fehlentwicklungen zeigt: ${ }^{263}$

- Es besteht ein Anreiz, die Zahl der Arztbesuche pro Behandlungsfall oder die Behandlungsintensität erheblich auszuweiten.

- Durch die häufigen Wiederbestellungen kommt es zu sehr kurzen Arzt-PatientenKontakten, die für den Patienten mit längeren Wartezeiten sowie Zeit- und Wegekosten sowie Belastungen am Arbeitsplatz verbunden sind.

- Die häufigen Wiederbestellungen üben wahrscheinlich eine expansive Wirkung auf die veranlassten Leistungen aus, weil ein Arztbesuch i.d.R. mit Verschreibungen von Arznei-mitteln, Massagen, der Nutzung medizinisch-technischer Leistungen u.a.m. verbunden ist.

\footnotetext{
${ }^{261} \mathrm{Vgl}$. Schulenburg (1981), S. 181.

${ }^{262}$ Vgl. im Folgenden Schulenburg (1981), S. $205 \mathrm{ff}$.

${ }^{263}$ Vgl. SVRKAiG (1988), Ziffer 119, S. 49f.
} 
- Eine Mengenausweitung erhöht bei vielen Untersuchungs- bzw. Behandlungsmethoden (z.B. Röntgenuntersuchungen oder Arzneimitteltherapien) das Risiko von gesundheitlichen Beeinträchtigungen durch unerwünschte Nebenwirkungen.

Positiv wird im Allgemeinen gesehen, dass die Einzelleistungsvergütung für die Patienten zu einer umfassenden diagnostischen und therapeutischen Versorgung führt. ${ }^{264}$ Voraussetzung ist allerdings, dass die erbrachten Leistungen in Bezug auf die Diagnose bzw. die Behandlung der jeweiligen Krankheit effektiv sind.

Wählt man den medizinischen Behandlungsfall als Grundlage der Vergütung, so besteht die Möglichkeit, eine Pauschale je eingeschriebenem Patienten (Kopfpauschale) oder je Behandlungsfall (Fallpauschale) zu zahlen. Im Kopfpauschalensystem sind die Patienten verpflichtet, sich zu Beginn einer Abrechnungsperiode bei einem Arzt ihrer Wahl einzuschreiben, den sie im Fall einer Erkrankung aufsuchen müssen. ${ }^{265}$ Diese Pauschale liefert dem Arzt grundsätzlich ein Interesse daran, die Größe seines Patientenstamms zu maximieren. ${ }^{266}$ Ferner besteht für den Arzt ein Anreiz, kostenminimale Faktorkombinationen und Behandlungen zu wählen. ${ }^{267} \mathrm{Da}$ die gesamten Aufwendungen aus dem Betrag beglichen werden müssen, den der Arzt vor der Periode erhält, wird er versuchen, die Kosten so gering wie möglich zu halten. Es existiert daher kein ökonomischer Anreiz, unwirksame oder schädlichen Leistungen zu erbringen.

Zusätzlich besitzt der Arzt ein Interesse daran, einen möglichst gesunden Patientenstamm zu versorgen, was zu einer stärker präventiven Ausrichtung der Behandlung beitragen kann, ${ }^{268}$ andererseits aber die Gefahr der Unterversorgung bestimmter Patienten birgt, da der angestrebte Behandlungserfolg im Wesentlichen von der moralischen Einstellung des Arztes zu seinem Beruf abhängt. Bei dieser Vergütungsform trăgt der Arzt das finanzielle Risiko einer steigenden Morbidität seines Patientenstamms. Er wird daher versuchen, möglichst solche Patienten zu attrahieren bzw. zu halten, die Kosten unterhalb der für sie gezahlten Kopfpauschale verursachen. Es fehlt deshalb bei einer einheitlichen Pauschale pro Patient ein finanzieller Anreiz, "kostspielige" Patienten angemessen zu behandeln. Durch eine Variation der Kopfpauschalen nach bestimmten Morbiditätsmerkmalen wie beispielsweise Alter und Geschlecht oder mittels Klassifikation nach Diagnosen ${ }^{269}$ kann der Gefahr einer Unterversorgung der entsprechenden Patientengruppen zwar entgegen gewirkt werden, ${ }^{270}$ doch eine Tendenz zur Risikoselektion durch die Ärzte wird sich nie ganz vermeiden lassen. Zudem ergibt sich für den Arzt der Anreiz, die Patienten möglichst schnell und u.U. ungerechtfertigt an die nächst höhere Versorgungsebene zu überweisen. ${ }^{271}$ Obwohl diese Vergütungsform die direkt von den Ärzten verursachten Kosten beschränkt, ist darum eine vollständige Kostenkontrolle aufgrund der durch nicht oder nur unzureichend durchge-

\footnotetext{
${ }^{264} \mathrm{Vgl}$. Reinhardt/Sandier/Schneider (1986), S. 19.

${ }^{265}$ Vgl. für eine ausfuhrliche Beschreibung der Anreizwirkungen Schulenburg (1981), S. 223ff.

${ }^{266}$ Vgl. Pfaff/Nagel (1995), S. 44; SVRKAiG (1988), Ziffer 131-132, S. 51.

${ }^{267}$ Vgl. Schulenburg (1981), S. 227; SVRKAiG (1988), Ziffer 131, S. 51.

${ }^{268} \mathrm{Vgl}$. Schulenburg (1981), S. 237.

${ }^{269} \mathrm{Vgl}$. Reinhardt/Sandier/Schneider (1986), S. 25.

${ }^{270} \mathrm{Vgl}$. SVRKAiG (1988), Ziffer 132, S. 51.

${ }^{271}$ Vgl. SVRKAiG (1988), Ziffer 131, S. 51.
} 
führte Behandlungen entstehenden Folgekosten sowie aufgrund des Weiterschiebens der Kosten auf andere Institutionen nicht möglich. ${ }^{272}$ Eine Möglichkeit, diese Überweisungen zumindest einzudämmen, besteht darin, dem Arzt für von ihm überwiesene $\mathrm{Pa}$ tienten nur einen Teil der Pauschale zu zahlen. ${ }^{273}$ Der Abschlag, den der Arzt hinnehmen muss, darf aber wiederum nicht so hoch sein, dass notwendige Überweisungen hinausgezögert werden oder unterbleiben.

In der Literatur findet sich das Argument, dass die im Kopfpauschalensystem bestehende Gefahr einer Unterversorgung der Patienten abgemildert wird, wenn ein intensiver Wettbewerb um Patienten herrscht: ${ }^{274}$ Wenn die Zahl der eingeschriebenen Patienten von der Leistung des Arztes in der Vorperiode abhängig ist, so wird der Arzt, auch wenn seine ärztliche Ethik eher schwach ausgeprägt ist, die Patienten möglichst gut behandeln, um sie im Wettbewerb nicht an Kollegen zu verlieren. ${ }^{275}$ Daraus würde folgen, dass neben einem guten Behandlungserfolg auch kurze Behandlungs- und Wartezeiten angestrebt werden. Problematisch ist jedoch, dass der Patient hier als souveräner Konsument gesehen wird, "[...] der Umfang, Inhalt und Angemessenheit medizinischer Dienstleistungen kompetent beurteilen kann. Dies ist jedoch in aller Regel nicht der Fall. Zudem verfügt der Arzt in der Regel über einen großen Spielraum, das Maß des medizinisch Notwendigen selbst zu definieren."1276

Als alleinige Vergütungsform ist die Kopfpauschale zudem ungeeignet, da sie eine Einschreibung der Patienten voraussetzt und damit die Fachärzte außen vor bleiben, die gänzlich oder überwiegend aufgrund von Überweisungen tätig werden. ${ }^{27}$

Eine Fallpauschale setzt stärker als die Kopfpauschale am tatsächlichen Behandlungsgeschehen an. Sie kann sich orientieren an

- der Zahl der tatsächlich behandelten Patienten,

- den angefangenen bzw. beendeten Behandlungen oder

- der Zahl aller innerhalb der Periode vorgenommenen Behandlungen. ${ }^{278}$

Alle drei Honorarformen haben aus allokativer Sicht den Vorteil, dass sie dem Arzt einen Anreiz bieten, einerseits die Einzelleistungen mit minimalem Ressourcenaufwand zu produzieren und andererseits (für einen gegebenen Behandlungserfolg) die Behandlung mit der geringst möglichen Zahl an Einzelleistungen zu wählen. ${ }^{279}$ Doch bietet die Fallpauschale, wie die Kopfpauschale, einen Anreiz zur Risikoselektion durch eine

\footnotetext{
${ }^{272}$ So. auch Pfaff/Nagel (1995), S. 44f.

${ }^{273} \mathrm{Vgl}$. SVRKAiG (1988), Ziffer 133, S. 52.

${ }^{274}$ Vgl. SVRKAiG (1988), Ziffer 131, S. 51.; Amold/Armann (1991), S. 31; Schulenburg (1981), S. 236.

${ }^{275} \mathrm{Vgl}$. Schulenburg (1981), S. 236ff.

${ }^{276}$ Gerlinger (1997), S. 44, Fn. 80.

${ }^{277}$ Vgl. Pfaff/Nagel (1995), S. 44; Schulenburg (1981), S. 243.

${ }^{278} \mathrm{Vgl}$. Schulenburg (1981), S. 181.

${ }^{279} \mathrm{Vgl}$. Schulenburg (1981), S. 251.
} 
Unterversorgung der „teuren“ Patienten und ungerechtfertigte Überweisungen ${ }^{280}$, verbunden mit den oben genannten Nachteilen für die Kostenkontrolle.

Weitere Nachteile entstehen durch die im Vergleich zur Kopfpauschale stärkeren Orientierung dieser Vergütungsformen am tatsächlichen Leistungsgeschehen, da sie den Leistungserbringern einen größeren Einfluss auf die Höhe der Vergütung verleihen. ${ }^{281}$ Anders als bei der Kopfpauschale steht die Gesamtvergütungssumme darum erst ex post fest und ist vom tatsächlichen Behandlungsgeschehen abhängig. Das Morbiditätsrisiko und auch das Risiko einer angebotsinduzierten Mengenausweitung tragen damit z.T. die Krankenkassen und somit die Versichertengemeinschaft. ${ }^{282}$ Werden als Bemessungskriterium die angefangenen bzw. beendeten Behandlungen oder die Zahl aller Behandlungen zugrunde gelegt, stellt zudem die klare Abgrenzung eines Behandlungsfalls ein Problem dar. So ist es denkbar, dass ein Patient innerhalb derselben Periode wegen zwei oder mehrerer Krankheiten behandelt wird, so dass der Arzt mehrere Fallpauschalen abrechnen kann. ${ }^{283}$ Hier bestehen für den Arzt tendenziell der Anreiz und die Möglichkeit zur Manipulation. Praktisch am leichtesten umzusetzen ist darum die Berechnung nach der Zahl aller behandelten Patienten.

Im Gegensatz zum Kopfpauschalensystem stehen Fallpauschalen einer präventiven Ausrichtung der Versorgung eher entgegen: Da der Arzt nur im Fall einer Erkrankung seines Patienten eine Vergütung erhält, besitzt er ein ökonomisches Interesse daran, dass dieser möglichst häufig leichte Krankheiten aufweist und vernachlässigt u.U. präventive Maßnahmen. So ist es bei einer Vergütung auf Basis der Anzahl aller behandelten Patienten sowie aller vorgenommenen Behandlungen für den Arzt ökonomisch rational, die Patienten zufrieden zu stellen, ohne einen optimalen Behandlungserfolg, d.h. eine schnelle Gesundung anzustreben, damit der Patient in der nächsten Abrechnungsperiode wieder in der Arztpraxis erscheint.

Wird die ärztliche Leistung in Form eines fixen Betrags pro Periode gezahlt, so kommt dies einer Anstellung des Arztes bei der zahlenden Institution gleich. ${ }^{284}$ Eine solche Honorierung in Form eines Gehalts bietet sich an, wenn die erbrachten Leistungen nicht nach Einzelleistungen oder Behandlungsfällen aufgegliedert werden können, beispielsweise weil Ärzte in Teams zusammenarbeiten oder wenn Ärzte zusätzlich in Lehre oder Forschung tätig sind. Welche Steuerungswirkungen von einer Gehaltszahlung ausgehen, hängt davon ab, inwieweit die Leistung eines Arztes Einfluss auf sein zukünftiges Einkommen ausübt. Besteht hier kein direkter Zusammenhang, fehlt der Anreiz, einen guten Behandlungserfolg zu erreichen, da die hierfür notwendigen zusätzlichen Anstrengungen und Aufwendungen nicht vergütet werden. Der Arzt wird also i.a. versuchen, seine Aktivität so gering wie möglich zu wählen. Als untere Gren-

\footnotetext{
${ }^{280} \mathrm{Vgl}$. SVRKAiG (1988), Ziffer 133, S. 52; Pfaff/Nagel (1995), S. 45.

${ }^{281} \mathrm{Vgl}$. Pfaff/Nagel (1995), S. 44.

${ }^{282}$ Dabei ist es aus ökonomischer Sicht sinnvoll, dass die Krankenkassen das Risiko einer steigenden Morbidität tragen, das damit auf die gesamte Versichertengemeinschaft übergeht. Problematisch ist aber, dass nur schwer feststellbar ist, ob Veränderungen im Leistungsgeschehen tatsächlich aus einer verănderten Morbidităt oder vielmehr aus einer ungerechtfertigten Mengenausweitung resultieren.

${ }^{283} \mathrm{Vgl}$. Schulenburg (1981), S. 254.

${ }^{284} \mathrm{Vgl}$. im Folgenden Reinhardt/Sandier/Schneider (1986), S. $26 \mathrm{f}$.
} 
ze existiert die ärztliche Ethik, also innere Wertmaßstäbe, die durch finanzielle Anreize nicht beeinflussbar sind. Dies führt tendenziell zu langen Wartezeiten, schnellen Überweisungen, ungünstiger Standortwahl, ungünstigen Öffnungszeiten und - wie oben bereits erläutert - einer schlechten Behandlungsqualität. ${ }^{285}$ Zudem besteht nur dann ein Anreiz zur kostenminimalen Produktion der Einzelleistungen, wenn der Arzt die Produktionskosten (teilweise) selbst tragen muss. Andererseits ergeben sich ähnliche Anreizwirkungen wie bei der Einzelleistungsvergütung, wenn das Gehalt vom Umfang der erbrachten Leistungen in der Vorperiode abhängt. Letztlich wird die Wirkung dieser Vergütungsform also stark von der Auswahl der Kriterien beeinflusst, die der Bemessung des Gehalts zugrunde liegen.

Die Darstellung der theoretischen Überlegungen zeigt, dass keines der vorgestellten Vergütungssysteme alleine zu optimalen Ergebnissen führt. Aus allokativer Sicht bietet die Vergütung mittels pauschalierter Zahlungen zwar den Vorteil, dass sowohl Produktions- als auch Behandlungseffizienz erreicht werden, da der Arzt ein wirtschaftliches Interesse daran hat, möglichst wenige Ressourcen einzusetzen. Doch gerade darin liegt auch der Nachteil dieses Systems. Wenn der Arzt überwiegend aufgrund finanzieller Anreize handelt - wovon auch bei Ärzten auszugehen ist - wird er versuchen, seinen Patientenstamm aus Personen zusammenzustellen, die ein unterdurchschnittliches Morbiditätsrisiko aufweisen. Vorteilhaft daran ist, dass der Arzt seine Medizin eher präventiv ausrichtet, wenn er sich daraus Kosteneinsparungen in der Zukunft erhofft. Allerdings droht Patienten eine Unterversorgung, wenn sie - beispielsweise aufgrund chronischer Krankheiten - überdurchschnittliche Kosten verursachen. Im Gegensatz dazu ist davon auszugehen, dass beim Einsatz der Einzelleistungsvergütung die Versorgung der Versicherten von besserer Qualität ist, da keine notwendigen Leistungen unterbleiben. Da das finanzielle Risiko einer Mengenausweitung hier bei den Krankenkassen liegt, ergibt sich aber ein Anreiz zu einer medizinisch nicht notwendigen Angebotssteigerung.

Auch die Ergebnisse empirischer Studien deuten darauf hin, dass ein Zielkonflikt zwischen Wirksamkeit und Wirtschaftlichkeit besteht. Vergütungssysteme, die Anreize zu einer qualitativ hochwertigen Versorgung bieten, führen zu einer Ausdehnung der Leistungen über das medizinisch notwendige Maß hinaus. Andererseits besteht bei einer Vergütung, die zu einer größeren Wirtschaftlichkeit in der Produktion der Gesundheitsleistungen führt, die Gefahr der Unterversorgung der Patienten. ${ }^{286}$ In der Realität sind die reinen Formen der Honorarsysteme selten anzutreffen. Sowohl das aktuelle als auch das geplante zukünftige deutsche System bestehen aus einer Kombination verschiedener Vergütungsregelungen.

\subsection{Vertragsärztliche Vergütung in Deutschland}

Das Sozialgesetzbuch schreibt kein explizites System für die Vergütung der ambulanten Ärzte durch die Krankenkassen vor. § 85 SGB V regelt lediglich die Zahlung einer

${ }^{285} \mathrm{Vgl}$. Schulenburg (1981), S. 193ff.

${ }^{286} \mathrm{Vgl}$. Reinhardt/Sandier/Schneider (1986), S. 12f. 
Gesamtvergütung an die jeweilige Kassenärztliche Vereinigung mit befreiender Wirkung. ${ }^{287}$ Diese Gesamtvergütung stellt das Ausgabenvolumen für die Gesamtheit der zu vergütenden vertragsärztlichen Leistungen in der jeweiligen Kassenärztlichen Vereinigung (KV) dar. ${ }^{288}$ Sie kann auf verschiedene Arten berechnet werden: als Festbetrag, auf Basis von Einzelleistungen, Kopfpauschalen, Fallpauschalen oder eines Systems, das sich aus der Verbindung dieser oder weiterer Berechnungsarten ergibt.

Derzeit zahlen die meisten Kassen eine Gesamtvergütung, die auf der Grundlage von „mitgliederbezogenen Kopfpauschalen“2689 errechnet wird. Die Kassenärztlichen Vereinigungen teilen diesen von den Kassen gezahlten Betrag auf die niedergelassenen Vertragsärzte ihres Bezirks auf. Als Schlüssel zur Verteilung auf die einzelnen Arztgruppen dient der Honorarverteilungsmaßstab (HVM), der seit dem GMG gemeinsam und einheitlich zwischen den KVen und den Landesverbänden der Krankenkassen sowie den Ersatzkassen zu vereinbaren ist. Zur Berechnung der individuellen Honorare setzt der Einheitliche Bewertungsmaßstab (EBM) die einzelnen Leistungen durch zugeordnete Punktwerte miteinander in Beziehung. Am Ende des Quartals wird die der KV zur Verfügung stehende Gesamtsumme für die Vergütung der jeweiligen Facharztgruppe durch die von allen Vertragsärzten der entsprechenden Gruppe erbrachte Punktemenge geteilt und das jeweilige Honorar des einzelnen Arztes berechnet, so dass der Arzt erst nach Ablauf der Periode die Höhe seiner Vergütung kennt. Einige Leistungen stellen dabei - entweder gesetzlich (z.B. Impfungen) oder vertraglich (z.B. Strukturverträge zum ambulanten Operieren) geregelte - Ausnahmen dar und werden mit festen Punktwerten und ohne Mengenbeschränkung vergütet. Das SGB V schreibt vor, dass die Gesamtvergütung jährlich nicht stärker wachsen darf als die beitragspflichtigen Einnahmen, um das Ziel der Beitragssatzstabilität nicht zu gefährden. ${ }^{290}$

Diese Art der Vergütung hat für die Krankenkassen den Vorteil, dass das finanzielle Risiko eines steigenden Behandlungsbedarfs - z.B. durch eine Zunahme der Morbidität oder eine anbieterinduzierte Nachfragesteigerung - von den Ärzten getragen wird und sich nicht auf die zu zahlende Gesamtvergütung auswirkt. Den Ärzten gibt dieses System jedoch auf individueller Ebene einen Anreiz zur Leistungsausdehnung: Bei gedeckelter Gesamtvergütung müssen die Punktwerte floaten, um sich an die jeweils erbrachte Leistungsmenge anzupassen. Für den einzelnen Arzt bedeutet dies, dass sein Einkommen zurückgeht, wenn die insgesamt von allen Ärzten der betreffenden KV erbrachte Leistungsmenge steigt, seine individuell abgerechnete Punktmenge aber konstant bleibt. Um dies zu verhindern, wird er versuchen, die von ihm erbrachte Leis-

\footnotetext{
${ }^{287} \mathrm{Vgl}$. im Folgenden insbesondere Rosenbrock/Gerlinger (2004), S. $121 \mathrm{ff}$.

${ }^{288}$ Vgl. § 85 Abs. 2 Satz 2 SGB V.

${ }^{289}$ Vgl. Jacobs (2002), S. 18.

${ }^{290}$ Vgl. § 85 Abs. 2 i.V.m. § 71 SGB V. Der Grundsatz der Beitragssatzstabilităt lässt jedoch Beitragssatzsteigerungen $\mathrm{zu}$, wenn die notwendige medizinische Versorgung auch unter Ausschöpfung von Wirtschaftlichkeitsreserven nicht ohne Beitragssatzerhöhungen zu gewährleisten ist. Zudem bestehen einige Ausnahmen, so dass beispielsweise Ausgabensteigerungen aufgrund gesetzlich vorgeschriebener Vorsorge- und Früherkennungsmaßnahmen nicht den Grundsatz der Beitragssatzstabilität verletzen.
} 
tungsmenge zu steigern ${ }^{291}$, um den Punktwertverfall zu kompensieren. Dieses individuell rationale Verhalten führt $\mathrm{zu}$ einer weiter steigenden Leistungsmenge und einem weiteren Punktwertverfall. Um diese Anreize zur Mengenausweitung einzudämmen, existierte ab dem Jahre 1997 zusätzlich zur sektoralen Budgetierung ein Praxisbudget als Obergrenze für die Gesamtsumme der abrechnungsfähigen Leistungen je Arzt, das jedoch zum 1. Juli 2003 wieder abgeschafft wurde. Eine Budgetierung für den einzelnen Arzt existiert jedoch weiterhin, wenn auf der KV-Ebene der jeweilige HVM ein sog. Individualbudget vorsieht.

Im Rahmen des Vergütungssystems ergaben sich durch das allgemeine Kassenwahlrecht der Versicherten Nachteile für die Vertragsärzte. Die Höhe der von den Kassen gezahlten Kopfpauschalen wurde erstmals mit dem GSG im Jahre 1992 festgesetzt und richtete sich nach den durchschnittlichen Leistungsausgaben der Kassen für die ambulante ärztliche Versorgung pro Mitglied im Jahre 1991 (für Westdeutschland) bzw. im 2. Halbjahr 1992 (für Ostdeutschland). ${ }^{292}$ Aufgrund unterschiedlicher Versichertenstrukturen der Kassen im Hinblick auf die Einkommensverhältnisse und das Krankheitsrisiko ergaben sich z.T. große Differenzen in den zu zahlenden Kopfpauschalen. Diese Struktur ist bis heute erhalten geblieben, obwohl sich die Mitgliederstruktur der einzelnen Kassen vor allem durch den Kassenwechsel zahlreicher Versicherter nach der Einführung des Kassenwahlrechts stark verändert hat. In der Tendenz wechseln vor allem junge und gesunde Versicherte die Krankenkasse, wobei die Höhe des Beitragssatzes eine wesentliche Entscheidungsgröße darstellt. ${ }^{293} \mathrm{Geht}$ man davon aus, dass der allgemeine Beitragssatz einer Krankenkasse und die von ihr gezahlte Kopfpauschale positiv korreliert sind, sinkt durch den Wechsel der Versicherten hin zu Kassen mit geringer Kopfpauschale die insgesamt zur Verfügung stehende Gesamtvergütung, obwohl sich der Behandlungsbedarf durch den Wechsel nicht ändert. Dies führt c.p. zu einem Sinken der Punktwerte und zu Einkommensverlusten bei den ambulanten Ärzten.

Darüber hinaus ergeben sich durch die Budgetierung des Ausgabenvolumens weitere grundlegende Probleme. So besteht die Gefahr, dass aufgrund mangelnder Flexibilität der Steuerungssysteme die Versorgungsstruktur des jeweiligen Basisjahres festgeschrieben wird, die langfristig nicht mehr mit dem tatsächlichen Leistungsgeschehen übereinstimmt. Zudem kann eine Budgetierung bestenfalls eine Kontrolle der Ausgaben erreichen; bei unzureichender Ausgestaltung führt die Budgetierung zu Qualitätsverschlechterungen und schafft Anreize zu ineffizientem Verhalten.

Aufgrund des Punktwertverfalls, der auch durch verschiedene mengenbegrenzende $\mathrm{Maßnahmen} \mathrm{nicht} \mathrm{gestoppt} \mathrm{werden} \mathrm{konnte,} \mathrm{besteht} \mathrm{für} \mathrm{die} \mathrm{Vertragsärzte} \mathrm{ein} \mathrm{Interesse}$ an einer Reform des Systems, die ihnen die Vergütung zu einem festen Punktwert bietet und somit eine größere Honorarsicherheit bringt. Zudem fordern die Ärzte den Ü-

${ }^{291}$ Voraussetzung hierfür ist, dass es dem Arzt möglich ist, entweder Leistungen zu übernehmen, die zuvor ein Kollege erbracht hat, oder einen zusätzlichen Behandlungsbedarf zu induzieren, der zuvor nicht vorhanden war.

${ }_{292}$ Vgl. Flintrop (2000), S. A 2976.

${ }^{293} \mathrm{Vgl}$. Schwarze/Andersen (2001). 
bergang des Morbiditätsrisikos auf die Versicherer, also die Krankenkassen. Die Krankenkassen erhoffen sich ihrerseits durch die Änderungen eine größere Transparenz, die im alten System nicht gegeben ist, da die gezahlten Kopfpauschalen nicht mehr dem tatsächlichen Behandlungsgeschehen entsprechen. Zudem waren im alten System die Inkompatibilitäten an der Schnittstelle zwischen ambulanten und stationären Versorgung ein Hindernis für integrierte Versorgungsformen.

Das GMG sieht daher einen Umbau des ambulanten Vergütungssystems vor. ${ }^{294} \mathrm{Ab}$ 2009 sollen die Gesamtvergütung und die Vergütung der einzelnen Ärzte auf Basis von morbiditätsorientierten Regelleistungsvolumina (RLV) berechnet werden. Der Einsatz dieser RLV ist auf allen Vergütungsebenen vorgesehen, wodurch die Honorarverteilungsmaßstäbe der KVen entfallen. Basis der RLV ist der auf Grundlage der Zahl und der Morbiditätsstruktur der Versicherten einer Krankenkasse jeweils ermittelte Behandlungsbedarf. Der zwischen den Vertragsparteien vereinbarte Behandlungsbedarf gilt laut Gesetz als notwendige medizinische Versorgung. ${ }^{295}$ Die Kassenärztlichen Vereinigungen und die Krankenkassen verteilen diesen Behandlungsbedarf nach zu vereinbarenden Kriterien auf die einzelnen Arztgruppen in Form von arztgruppenbezogenen RLV, die von den Vertragsparteien der Gesamtverträge jährlich angepasst werden sollen. Die Zahlung der Gesamtvergütung der Kassen erfolgt weiterhin mit befreiender Wirkung, so dass die über die RLV hinausgehenden Leistungen von ihnen grundsätzlich nicht erstattet werden. Eine Ausnahme besteht, wenn die Leistungsmenge aufgrund eines bei der Vereinbarung nicht vorhersehbaren Anstiegs des morbiditätsbedingten Behandlungsbedarfs zunimmt. Diese Leistungen sind mit einem auf 10 Prozent des Regelpunktwerts abgestaffelten Punktwert zu vergüten.

Als letzten Schritt bestimmen die KVen auf Grundlage einer Vereinbarung mit den Landesverbänden der Krankenkassen arztbezogene RLV sowie deren Zuweisung auf die an der vertragsärztlichen Versorgung teilnehmenden Ärzte. Dieses Volumen gibt die vom Arzt innerhalb eines bestimmten Zeitraums abrechenbare Leistungsmenge an, die mit dem festen Regelpunktwert vergütet wird. ${ }^{296}$ Die Summe der arztbezogenen RLV ist dabei nicht mit dem zugehörigen arztgruppenbezogenen RLV identisch. Leistungen, die über das Regelleistungsvolumen hinausgehen, werden - anders als auf der Ebene der Gesamtvergütung - generell mit einem auf 10 Prozent des Regelpunktwerts abgestaffelten Punktwert vergütet. Hiervon kann abgewichen werden, wenn es zu einer außergewöhnlich starken Erhöhung der Zahl der durch den Arzt behandelten Versicherten kommt.

Diese Form der Vergütung stellt für die Ärzte bis zur Grenze des Regelleistungsvolumens eine Einzelleistungsvergütung mit festen Punktwerten dar. Durch die starke Abstaffelung des Punktwerts für Leistungen, die über diese Grenze hinausgehen, soll den Ärzten aber der mit der Einzelleistungsvergütung verbundene Anreiz zur "übermäßigen" Mengenausweitung genommen werden. Eine Steuerung der GKV-Ausgaben für den ambulanten Bereich ist bei festen Punktwerten notwendigerweise nur über die

\footnotetext{
${ }^{294} \mathrm{Vgl}$. Abs. 65 GMG; § 85a und b SGB V.

${ }^{295}$ Vgl. $\$ 85$ a Abs. 4, Satz 2 SGB V.

${ }^{296} \mathrm{Vgl}$. $84 \mathrm{~b}$ Abs. 2 SGB V.
} 
Festlegung der maximal abrechenbaren Leistungsmenge möglich. Dies kann zu einer impliziten Rationierung durch die Ärzte führen: Ist ihr Regelleistungsvolumen ausgeschöpft, besteht für sie i.d.R. kein finanzieller Anreiz, darüber hinaus Leistungen zu erbringen, da davon auszugehen ist, dass der auf 10 Prozent des Regelpunktwertes abgestaffelte Punkwert für die meisten Leistungen nicht kostendeckend sein dürfte. Damit ist nicht auszuschließen, dass medizinisch notwendige Leistungen in die nächste Abrechnungsperiode verschoben werden oder ganz unterbleiben. Eine solche Verschiebung von Leistungen kann zu einer Unterversorgung der Bevölkerung führen. Diese Gefahr ist nur dann nicht gegeben, wenn der zwischen den Vertragsparteien vereinbarte Behandlungsbedarf, der laut Gesetz als notwendige medizinische Versorgung gilt, den tatsächlichen Bedarf hinreichend genau abbildet. $\mathrm{Ob}$ dies ex ante überhaupt möglich ist, bleibt fraglich.

Andererseits kann im Konsens der Vertragsparteien durch die Vereinbarung eines höheren Behandlungsbedarfs von der gesetzlich verankerten Grundlohnsummenbindung abgewichen werden, was dem Grundsatz der Beitragssatzstabilität zuwiderlaufen würde. Problematisch dabei ist, dass die Ärzte damit aus theoretischer Sicht die Möglichkeit besitzen, den festgelegten Behandlungsbedarf zu beeinflussen. Die für die Vereinbarung des Behandlungsbedarfs zwischen Kassen und KVen vorgeschriebene Eingruppierung der Patienten in Risikoklassen erfolgt auf Basis verschiedener Risikomerkmale, wie Alter und Geschlecht sowie der von den Ärzten gestellten Diagnosen. Dabei besteht die Gefahr, dass die Ärzte im Rahmen der Diagnosestellung versuchen werden, ihre Patienten in möglichst hohe Risikoklassen einzuordnen („Upcoding“), um durch den zu beobachtenden Morbiditätsanstieg eine Begründung für entsprechende Honorarforderungen zu erhalten. ${ }^{297} \mathrm{Da}$ der Regelpunktwert längerfristig unverändert bleiben soll, führt ein steigender Behandlungsbedarf zwingend zu einem steigenden Honorar der Vertragsärzte und damit zu steigenden Ausgaben der Kassen in der vertragsärztlichen Versorgung. Durch die Morbiditätsorientierung des Systems und den damit verbundenen Übergang des Morbiditätsrisikos auf die Krankenkassen übernehmen diese nun auch das finanzielle Risiko einer anbieterinduzierten Nachfragesteigerung.

\subsection{Europatauglichkeit der betrachteten Systeme und Ände- rungsvorschläge}

In Folge der EuGH-Urteile in den Rechtssachen „Koh11“ und „Decker“2098 wurde vielfach vermutet, dass das bestehende Vergütungssystem sowie die damit verbundene Budgetierung des Honorars bei einer Umsetzung der Urteile in Deutschland nicht aufrechterhalten werden könne. ${ }^{299}$ Die bisherige Entwicklung bestätigte diese Vermutung nicht, da die Patientenmobilität ein nur sehr geringes Ausmaß erreichte. Die nachfolgenden Betrachtungen sollen jedoch Auskunft darüber geben, welche Auswirkungen

\footnotetext{
${ }^{297}$ So auch Staffeldt (2004), S. 52.

${ }^{298} \mathrm{Vgl}$. zur Darstellung dieser Urteile Abschnitt 2.3.3.1.

${ }^{299}$ Vgl. z.B. Münnich (1998), S. $129 f$.
} 
bei einer signifikant zunehmenden Auslandsnachfrage deutscher Patienten zu erwarten sind. Die bevorstehende Reform der vertragsärztlichen Vergütung macht es dabei nötig, auch das geplante System in die Betrachtung einzubeziehen. Dabei ergibt sich auch die Gelegenheit zu überprüfen, inwieweit die Politik die zunehmende Europăisierung der Gesundheitsmärkte bei Veränderungen der gesetzlichen Rahmenbedingungen mit einbezieht.

Leistungen im ambulanten Bereich, die Versicherte außerhalb Deutschlands in Anspruch nehmen - unabhängig davon, ob dies mit oder ohne vorherige Genehmigung geschieht - sind laut Bundesmantelvertrag Ärzte ( $\$ 3$ Abs. 2 Nr. 9) bzw. ArztErsatzkassen-Vertrag ( $\$ 2$ Abs. 12 Nr. 9) keine Leistungen der vertragsärztlichen Versorgung und werden daher nicht in die Gesamtvergütung einbezogen. ${ }^{300}$ Die Krankenkassen erstatten diese Leistungen zusätzlich zur Vergütung der inländischen Ärzte. Auch wenn es aufgrund einer zunehmenden Auslandsnachfrage zu einem Rückgang der im Inland erbrachten Leistungen käme, wäre damit im derzeitigen System eine Ausgabensteigerung im ambulanten Bereich verbunden, wenn die Kassen - wie realistischerweise anzunehmen ist - keine Absenkung der Gesamtvergütung durchsetzen könnten. Ein Nachfragerückgang im Inland bedeutet c.p. eine Steigerung des Punktwertes für die erbrachten Leistungen, von dem insbesondere diejenigen Ärzte profitierten, die keine Reduktion ihrer individuellen Leistungsmenge erleiden würden. Da jedoch die Ärzte, die von der Verschiebung der Nachfrage in größerem Maße betroffen wären, einen Einkommensverlust hinnehmen müssten, besteht für sie der Anreiz, mit einer Angebotsinduktion zu reagieren, so dass letztlich die im Inland erbrachte Leistungsmenge kaum sinken würde. Insgesamt ergäbe sich dann eine deutliche Zunahme der im In- und Ausland insgesamt erbrachten Leistungsmenge, die eine spürbare Ausgabensteigerung verursachen würde. Es stellt sich beim derzeitigen System darum die Frage, ob eine Einbeziehung der ausländischen Leistungserbringer in die Budgetierung möglich ist.

Im grenznahen Ausland könnte dies durch den Abschluss von Verträgen mit den dort ansässigen Leistungserbringern geschehen. ${ }^{301}$ Es ist davon auszugehen, dass der wesentliche Teil der Auslandsnachfrage in diesen Gebieten anfällt. Die Zahl der Kostenerstattungsfälle würde unter diesen Umständen gering bleiben, weshalb die Kassen hierfür entsprechende Rücklagen bilden könnten, die bei der Vereinbarung der Gesamtvergütung Berücksichtigung fänden. Es gilt in diesem Kontext allerdings zu beachten, dass derartige Regelungen Reaktionen der inländischen Vertragspartner erwarten lassen, die auf eine Erhöhung der Budgets drängen, was letztendlich zu einer Beitragserhöhung führen könnte. Ökonomisch ist eine solche Erhöhung nicht zwingend nötig, da die Möglichkeit der Inanspruchnahme im Ausland nicht zu einer steigenden Gesamtnachfrage nach medizinischen Leistungen führen muss. Letztlich hängt das Ergebnis jedoch von der jeweiligen Verhandlungsposition der Vertragspartner ab.

Diese Überlegungen erlangen allerdings erst dann Relevanz, wenn die Inanspruchnahme von Auslandsleistungen ein wesentlich größeres Ausmaß einnimmt, als es der-

${ }^{300} \mathrm{Vgl}$. auch im Folgenden Jacobs/Wasem (2003), S. 30ff.

${ }^{301} \mathrm{Vgl}$. Wille (1998), S. $352 \mathrm{f}$. 
zeit der Fall und für die nähere Zukunft zu erwarten ist. Aufgrund der geplanten Umstellung der vertragsärztlichen Vergütung ist daher von größerer Bedeutung, inwieweit sich das neue System als europatauglich erweist.

Die Kontrolle der Ausgabenentwicklung im ambulanten Bereich erfolgt in diesem neuen System durch eine Steuerung der Menge in Form der Vorgabe eines Behandlungsbedarfs. Die Berücksichtigung einer stark zunehmenden Nachfrage nach ambulanten Leistungen im Ausland ist dabei grundsätzlich möglich. Es ist davon auszugehen, dass von einer derartigen Entwicklung nicht alle KVen gleichermaßen betroffen wären, sondern insbesondere KV-Bezirke in grenznahen Gebieten Nachfragerückgänge verzeichnen würden. Eine wirksame Ausgabenbegrenzung setzt voraus, dass der vereinbarte inländische Behandlungsbedarf in diesen Bezirken entsprechend der Inanspruchnahme im Ausland vermindert wird. Dabei ist jedoch von ähnlichen Schwierigkeiten auszugehen wie bei der oben diskutierten Reduktion der inländischen Gesamtvergütung. Allerdings ist zu berücksichtigen, dass sich eine derartige Entwicklung nur schrittweise ergeben wird. Geht man davon aus, dass der vereinbarte Behandlungsbedarf in Zukunft ohnehin tendenziell ansteigen wird, könnten die Kassen in den genannten Gebieten mit dem Argument einer beobachteten zunehmenden Patientenmobilität einen geringeren Anstieg der vereinbarten Menge erreichen als es im Durchschnitt der KVen der Fall ist. Voraussetzung hierfür sind ausreichende Informationen über die tatsächlichen Patientenwanderungen.

Grundsätzlich bleibt aber festzuhalten, dass eine Einbeziehung ausländischer Ärzte in die vorgestellten Systeme nur durch systemfremde Elemente (z.B. Einzelverträge oder Kostenerstattung) möglich ist. Dies zeigt, dass das Problem der grenzüberschreitenden Inanspruchnahme von Gesundheitsleistungen bei politischen Entscheidungen über Reformen der vertragsärztlichen Vergütung unberücksichtigt bleibt. Aufgrund der sehr geringen Fallzahlen ergeben sich daraus bisher keine spürbaren Auswirkungen auf die inländischen Akteure. Aus ökonomischer Sicht ist dennoch eine grundlegende Änderung der vertragsärztlichen Vergütung wünschenswert. Sowohl das derzeitige als auch das geplante System weisen eine hohe Regulierungsdichte auf, die wettbewerbliche Elemente aus der vertragsärztlichen Vorsorgung weitgehend ausschließt und damit einen Preis- und/oder Qualitätswettbewerb verhindert. Da auf diese Weise das angestrebte Ziel einer wirtschaftlichen Erbringung qualitativ hochwertiger Leistungen offenbar nicht sichergestellt werden kann, ist eine grundsätzliche Strukturveränderung erstrebenswert. Eine europakonforme Lösung stellt dabei ein System individueller Verträge zwischen Ärzten und Krankenkassen dar, das darüber hinaus die Inanspruchnahme von Nicht-Vertragsärzten mittels Kostenerstattung erlaubt. Dieses System behandelt inländische und ausländische Ärzte gleich und baut Hindernisse bei der grenzüberschreitenden Inanspruchnahme von Gesundheitsleistungen ab. Eine wachsende Patientenmobilität stellt hier kein grundsätzliches Problem dar, weil Einzelverträge (ohne Kontrahierungszwang) den Kassen die Möglichkeit geben, flexibler auf Veränderungen im Leistungsgeschehen zu reagieren. Auf diese Weise könnte auch die Bedarfsplanung entfallen, die bei einer stark wachsenden Patientenmobilität ohnehin an Wirksamkeit verliert. Zudem ergäbe sich ein Wettbewerb unter den Leistungserbringern um Verträge mit den Krankenkassen sowie vice versa der Krankenkassen um 
Verträge mit guten Leistungserbringern, der auch grenzüberschreitend Wirkung zeigen und eine weitere Europäisierung der Gesundheitsmärkte fördern würde.

\subsubsection{Veranlasste Leistungen}

Die Höhe der GKV-Ausgaben für Arznei-, Heil- und Hilfsmittel wird wesentlich durch das Verordnungsverhalten der niedergelassenen Ärzte bestimmt. Darum setzen verschiedene Maßnahmen zur Mengensteuerung dieser sog. veranlassten Leistungen bei den ambulant tätigen Ärzten an. In Deutschland existierte von 1993 bis 2000 ein Budget für die GKV-Arzneimittelausgaben (einschließlich Heilmittel). ${ }^{302}$ Die Höhe der Kostenobergrenze für die von den Vertragsärzten verordneten Leistungen verhandelten die Landesverbände der Krankenkassen jährlich mit den KVen. Wenn die niedergelassenen Ärzte einer KV die Grenze überschritten, sollte sich die ärztliche Gesamtvergütung in diesem Bereich - bis zu einer Obergrenze - um den Fehlbetrag verringern, so dass die Ärzte einer KV kollektiv für die hohen Ausgaben gehaftet hätten. Die Umsetzung dieses Kollektivregresses stellte sich problematisch dar und warf juristische Probleme auf, so dass er trotz wiederholter Budgetüberschreitungen nie zur Anwendung kam. Aus ökonomischer Sicht ergaben sich durch diese Regelungen Anreizprobleme, da das Budget auf der Ebene der KVen bestimmt wurde, obwohl die tatsächliche Ausgabenentscheidung beim einzelnen Arzt lag und zudem mit der Kollektivhaftung kein wirksamer Sanktionsmechanismus existierte. Wäre es tatsächlich zu Sanktionen gekommen, wären auch Ärzte mit wirtschaftlichem Verschreibungsverhalten bestraft worden. Insgesamt gingen damit von der Budgetierung nicht die gewünschten steuernden Anreize aus. ${ }^{303}$

Mit dem Gesetz zur Ablösung des Arznei- und Heilmittelbudgets (ArzneimittelbudgetAblösegesetz, ABAG) wurde die Budgetierung zum 1.1.2002 abgeschafft und durch arztindividuelle Richtgrößen ${ }^{304}$ ersetzt, die getrennt für Arznei- und Heilmittel vereinbart werden. Die KVen vereinbaren jährlich mit den zuständigen Verbänden der Krankenkassen

- das Ausgabenvolumen für die insgesamt von den Vertragsärzten veranlassten Leistungen,

- Versorgungs- und Wirtschaftlichkeitsziele und konkrete, auf die Umsetzung dieser Ziele ausgerichtete Maßnahmen - insbesondere zur Information und Beratung sowie

- Kriterien für Sofortmaßnahmen zur Einhaltung des vereinbarten Ausgabevolumens im laufenden Jahr sowie

- einheitliche arztgruppenspezifische Werte als Durchschnittswerte, die das Volumen der je Vertragsarzt verordnungsfähigen Leistungen festlegen.

\footnotetext{
${ }^{302}$ Vgl. im Folgenden Rosenbrock/Gerlinger (2004), S $177 \mathrm{ff}$.

${ }^{303}$ Die deutliche Unterschreitung des Budgets um ca. 2 Mrd. DM in Westdeutschland im Jahr 1993 ist wohl überwiegend auf einen psychologischen Effekt zurückzuführen. Vgl. o.V. (1998a).

${ }^{304} \mathrm{Vgl}$. 84 SGB V.
} 
Die Einhaltung der Richtgrößen kann im Rahmen von Wirtschaftlichkeitskontrollen überprüft werden. Überscheitet ein Arzt die für ihn geltende Richtgröße um mehr als 25 Prozent, muss er den Mehraufwand erstatten, wenn er keine Praxisbesonderheiten als Begründung anführen kann. Diese Zahlungen können bei einer wirtschaftlichen Gefährdung des Arztes allerdings gestundet oder erlassen werden. Das GMG verstärkte den Anreiz zur Einhaltung der Zielvereinbarungen, indem es Vereinbarungen über einen prozentualen Betrag der Gesamtvergütung möglich macht, von dem Bonuszahlungen an die Vertragsärzte geleistet werden können, die ihr Richtgrößenvolumen einhalten. ${ }^{305}$ Diese Boni können einen Nachteil der in Deutschland geltenden Richtgrößen ausgleichen: $\mathrm{Da}$ die Richtgrößen gewissermaßen ein virtuelles Budget darstellen, haben sie gegenüber realen Budgets den Nachteil, dass der verordnende Arzt für eine Unterschreitung des Budgets nicht damit belohnt wird, dass er den Differenzbedarf als zusätzliches Einkommen erhält. ${ }^{306}$

Grundsätzlich gilt es bei einer Budgetierung bestimmter Leistungen zu beachten, in welcher Form für den verordnenden Arzt Ausweichoptionen bestehen. Beispielsweise besteht als Alternative zu einer Arzneimittelverordnung u.U. die Möglichkeit, eine nicht-medikamentöse Behandlung zu wählen, die mit der Verordnung von Heilmitteln einhergehen kann. Diese Option ist für den Arzt interessant, wenn in diesem Bereich kein bzw. ein separates weniger restriktives Budget besteht. Problematisch ist dieses Verordnungsverhalten, wenn es sich nicht an der medizinischen Notwendigkeit orientiert, sondern vorrangig den wirtschaftlichen Interessen des Arztes dient. Neben der Wahl einer alternativen Therapie gehört prinzipiell auch die Überweisung - insbesondere kostenintensiver Fälle - an einen anderen Arzt oder an den stationären Sektor zu den Handlungsoptionen des Arztes. Bei einer sektoralen Budgetierung entlastet eine Überweisung in einen anderen Sektor (z.B. ins Krankenhaus oder vom Hausarzt an einen Facharzt) neben dem Arznei- oder Heilmittelmittelbudget auch ein eventuell bestehendes Honorarbudget. ${ }^{307}$

Ein Budget ist dann ideal ausgestaltet, wenn es den Leistungserbringer dazu anreizt, in medizinisch notwendigen Fällen möglichst preisgünstige Arznei- oder Heilmittel zu verordnet und unnötige Verordnungen zu unterlassen. ${ }^{308}$ In Bezug auf Arzneimittel stellte der Sachverständigenrat zur Begutachtung der Entwicklung im Gesundheitswesen fest, dass eine Budgetierung der Ausgaben die Steuerung der Ausgabenzuwächse ermöglicht, wenn die folgenden wesentlichen Elemente Berücksichtigung finden:

\footnotetext{
${ }^{305} \mathrm{Vgl}$. 884 Abs. 4a SGB V

${ }^{306} \mathrm{Vgl}$. Sachverständigenrat zur Begutachtung der Entwicklung im Gesundheitswesen (2005), Rn. 808.

${ }^{307}$ Diesem Problem versuchen verschiedene in Deutschland existierende Modellversuche einer sog. „Integrierten Versorgung“ zu begegnen. In einem Netz zwischen den einzelnen medizinischen Versorgungssektoren bieten niedergelassene Haus- oder Fachärzte mit stationären Einrichtungen eine gemeinsame medizinische Versorgung an.

${ }^{308} \mathrm{Vgl}$. Sachverständigenrat zur Begutachtung der Entwicklung im Gesundheitswesen (2005), Rn. 809.
} 
- „die Individualisierung von Budgets,

- die Sanktionierung von Budgetüberschreitungen,

- das Ermöglichen von Überschreitungen in begründeten Ausnahmefällen,

- die zeitnahe Bereitstellung von Information zum individuellen Verordnungsverhalten,

- das Vermeiden von Nachteilen durch wirtschaftliches Verhalten,

- das Berücksichtigen von Handlungsalternativen des Arztes durch eine sektorenund therapieübergreifende Perspektive und

- der Einsatz von flankierenden qualitätssichernden Maßnahmen zur Vermeidung von Unter- und Fehlversorgung. ${ }^{\text {.309 }}$

Die Ausgestaltung der derzeit geltenden Richtgrößen bietet in dieser Hinsicht deutliche Vorteile gegenüber den früheren Budgets in Verbindung mit Kollektivregressen. Wie sind diese Regelungen jedoch im Kontext einer wachsenden Inanspruchnahme ausländischer Leistungserbringer zu bewerten? Unterschieden werden müssen dabei zwei relevante Fälle: Ein in Deutschland niedergelassener Arzt verordnet ein Medikament oder Heilmittel, das der Patient in einer Apotheke oder bei einem Leistungserbringer im europäischen Ausland einlöst bzw. der Patient erhält ein Rezept von einem ausländischen Arzt, wobei es in diesem Fall unerheblich ist, ob er es in Deutschland oder im Ausland einlöst.

Im Jahre 2001 stellte das deutsche Gesundheitsministerium in einer „Mitteilung der Bundesrepublik Deutschland an die Kommission der Europäischen Union" fest, dass ein „Anspruch der Versicherten auf Kostenerstattung für in Apotheken der Mitgliedstaaten aufgrund deutscher Rezepte erworbenen Arzneimitteln besteht und dieser Anspruch nicht mehr unter Hinweis auf das geltende Sachleistungsprinzip abgelehnt werden darf." Dies gilt analog auch für von inländischen Ärzten verordnete Heil- und Hilfsmittel. Begrenzt ist diese Erstattungspflicht auf Medikamente und Leistungen, für die in Deutschland eine Leistungspflicht der GKV besteht. Die Höhe des Erstattungsbetrags entspricht dabei maximal der Summe, die die deutsche Krankenkasse bei einem Erwerb in Deutschland dafür aufgewendet hätte. Für Arzneimittel übernehmen die Kassen die Kosten maximal bis zur Höhe der deutschen Apothekenabgabepreise bzw. der geltenden Festbeträge abzüglich der in Deutschland fälligen Zuzahlungsbeträge sowie dem Kassenrabatt, jedoch nicht mehr als die tatsächlich angefallenen Kosten. Die Ausgaben für die im Ausland in Anspruch genommenen Heilmittel werden ebenfalls maximal bis zur Höhe der in Deutschland anfallenden Kosten ersetzt. Die entsprechenden Ausgaben werden auf das Regelleistungsvolumen des verordnenden Arztes angerechnet. Falls die dabei entstehenden Kosten geringer sind als bei einer Inanspruchnahme im Inland - was insbesondere bei im Ausland erworbenen Medikamenten der Fall sein kann - entsteht für den Arzt ein größerer finanzieller Spielraum für die Verordnung von veranlassten Leistungen.

${ }^{309}$ Sachverständigenrat zur Begutachtung der Entwicklung im Gesundheitswesen (2005), Rn. 813. 
Auch wenn ein ausländischer Arzt Leistungen verordnet, übernehmen die deutschen Krankenkassen deren Kosten, wenn der Arzt im ausländischen System zur Versorgung von Patienten zugelassen ist. Eine Einbeziehung dieser Leistungen in die Richtgrößen der deutschen KVen und Ärzte ist jedoch schon deshalb ausgeschlossen, weil keine individuelle Zuordnung möglich ist. Kommt es zu einer wachsenden Inanspruchnahme ausländischer Ärzte durch deutsche Patienten und geht diese mit einem Nachfragerückgang im Inland einher, der auch zu einem geringeren Verordnungsvolumen führt, besteht bei Richtgrößen gegenüber einer fixen Budgetierung jedoch der Vorteil, dass dies i.d.R. automatisch zu geringeren Kosten für veranlasste Leistungen führt, da die Kassen kein fixes Budget zahlen, sondern nur die tatsächlichen Verordnungen erstatten. ${ }^{310}$ Eine stärkere finanzielle Belastung der Krankenkassen infolge der Nachfrageverschiebung ins Ausland ist in diesem Szenario denkbar, wenn es ohne die Verschiebung zu einer so massiven Überschreitung der Arzneimittelrichtgrößen auf KV-Ebene oder bei einzelnen Ärzten gekommen wäre, dass die Ärzte Regresszahlungen an die Kassen hätten leisten müssen. Fallen diese Zahlungen aufgrund der Abwanderung der Nachfrage weg und kompensieren die im Ausland anfallenden Kosten die Kosteneinsparungen im Inland, müssen die Kassen dennoch die gesamten Arzneimittelausgaben im In- und Ausland tragen, ohne dass sie durch eine Regresszahlung von Seiten der Ärzten entlastet werden. ${ }^{311}$ Auch wenn die inländischen Ärzte auf einen spürbaren Rückgang der Inlandsnachfrage mit einer Angebotsinduktion reagieren, kann diese zu insgesamt höheren Ausgaben für veranlasste Leistungen führen. ${ }^{312}$ Hierbei ist aber zu berücksichtigen, dass sich für die Ärzte aus einer Nachfrageverschiebung, die sich lediglich auf die veranlassten Leistungen bezieht, keine finanziellen Nachteile ergeben. Eine Angebotsinduktion ihrerseits ist darum wenig wahrscheinlich. Hinzu kommt, dass die Anbieter der Leistungen selbst keine Möglichkeit besitzen, eine zusätzliche Nachfrage zu generieren. Für die Krankenkassen besteht darum vor allem dann ein Interesse, die im Ausland verordneten Leistungen bei der Vereinbarung der inländischen Richtgrößen und Zielvereinbahrungen zu berücksichtigen, wenn es zu einer deutlichen Verschiebung der Nachfrage nach ambulanten ärztlichen Leistungen ins Ausland und einer damit einhergehenden steigenden Verordnung veranlasster Leistungen kommt. Es ist jedoch zu erwarten, dass KVen und Ärzte eine Reduktion der Richtgrößen nicht ohne weiteres hinnehmen werden, da sie eine Einschränkung ihrer Handlungsfreiheit befürchten. Interessant ist in diesem Zusammenhang, dass die Zielvereinbarungen für das nächste Jahr zwischen KVen und Krankenkassen bis zum 30. November des vorhergehenden Jahres vereinbart sein müssen. Ansonsten entscheidet das zuständige Schiedsamt bzw. bei Klagen gegen den Schiedsspruch das Bundessozialgericht über die Höhe der Richtgrößen. Auf dem juristischen Weg könnte daher auch gegen einen eventuellen Widerstand der Ärzte tatsächlich eine Berücksichtigung der im Ausland verordneten Arzneimittel bzw. Heilmittel erreicht werden, wenn sie einen spürbaren Umfang erreichen.

\footnotetext{
310 Ähnlich Domscheit (1998), S. 248.

${ }^{311} \mathrm{Vgl}$. Jacobs/Wasem (2003), S. 52.

${ }^{312}$ Aus diesem Grund ist die Anreizwirkung des Vergütungssystems der ambulant tătigen Ärzte auch im Bereich der veranlassten Leistungen von großer Bedeutung.
} 


\subsubsection{Stationäre Versorgung}

Die stationäre Versorgung in Deutschland wird zum einen erbracht von Krankenhäusern, die die Akutbehandlung der Patienten übernehmen, wenn eine dauerhafte Unterbringung und medizinische Überwachung notwendig ist, zum anderen von Vorsorgeund Rehabilitationseinrichtungen, die im Bereich der Prävention und Nachsorge tätig sind.

\subsubsection{Krankenhäuser}

Der Krankenhaussektor war in den vergangenen Jahrzehnten strukturellen Veränderungen unterworfen, die sich auch in den Daten zur Krankenhausversorgung in Deutschland niederschlagen (vgl. Tabelle 1). Die Zahl der Krankenhäuser lag 2003 mit 2.197 um fast 9 Prozent niedriger als 1991 (2.411 Kliniken). Im gleichen Zeitraum sank die Bettenzahl von 665.565 auf 541.901 - also um gut 18 Prozent - und die durchschnittliche Bettenauslastung nahm von 83,8 (1991) auf 77,6 Prozent (2003) ab, was mit einer deutlichen Abnahme der durchschnittlichen Verweildauer einherging. Gleichzeitig ist eine Zunahme der stationären Behandlungsfälle zu beobachten, deren Ursache in einer steigenden Morbidität aber auch in den Anreizwirkungen des Fallpauschalensystems liegen kann. Diese äußern sich darin, dass Patienten (vorzeitig) entlassen und wieder aufgenommen werden, um auf diese Weise eine Fallpauschale mehrmals abrechnen zu können („Fall-Splitting“). ${ }^{313}$ Seit der Verordnung zum Fallpauschalensystem für Krankenhäuser für das Jahr 2004 (KVPF 2004) sind einem solchen Verhalten jedoch Grenzen gesetzt. \$2 der Verordnung regelt für bestimmte Fälle eine Zusammenlegung von zwei oder mehreren Aufenthalten zu einem Gesamtfall. Im internationalen Vergleich weist Deutschland - trotz der eben beschriebenen Entwicklungen - jedoch immer noch eine hohe Bettenkapazität und eine überdurchschnittliche Verweildauer auf. ${ }^{314}$

Der stationäre Sektor verursacht den größten Anteil der Leistungsausgaben der GKV. Dieser Anteil ist in der Vergangenheit von 25,2 Prozent im Jahre 1970 auf 34,3 Prozent im Jahre 2003 angestiegen. ${ }^{315}$ Auch die Zahl der Ärzte wuchs im stationären Sektor schneller als im ambulanten. Damit bilden diese Zahlen den Bedeutungszuwachs des stationären Sektors gegenüber der ambulanten Versorgung ab. Die Kostenentwicklung im stationären Bereich hat dazu geführt, dass die GKV-Ausgaben für die stationäre Versorgung seit 1993 einer Budgetierung unterliegen, die sich sowohl auf den gesamten Sektor als auch auf jedes einzelne Krankenhaus bezieht. Mit der Einführung eines „durchgängigen, leistungsorientierten und pauschalierten“316 Vergütungssystems, das auf diagnosebezogenen Fallpauschalen (Diagnosis Related Groups (DRGs)) beruht und seit 2004 schrittweise eingeführt wird, ist die Erwartung verbunden, dass

\footnotetext{
${ }^{313} \mathrm{Vgl}$. Rosenbrock/Gerlinger (2004), S. 141.

${ }^{314} \mathrm{Im}$ OECD-Durchschnitt lag die Verweildauer im Jahr 2000 bei rund 6,9 Tagen.

${ }^{315} \mathrm{Vgl}$. Verband der Angestellten-Krankenkassen (VdAK) (2006); eigene Berechnungen.

${ }^{316} \mathrm{Vgl}$. § 17 Abs. 1 Krankenhausfinanzierungsgesetz (KHG).
} 
wettbewerbliche Elemente im stationären Sektor an Bedeutung gewinnen und die Budgetierung ab dem Jahre 2007 entfallen kann. ${ }^{317}$

Tabelle 1: Eckzahlen der stationären Versorgung in Deutschland von 1991 bis 2003

\begin{tabular}{|l|l|l|l|l|l|l|}
\hline & $\begin{array}{l}\text { Kranken- } \\
\text { häuser }\end{array}$ & Betten & $\begin{array}{l}\text { Betten je } \\
10.000 \\
\text { Einwohner }\end{array}$ & Patienten & $\begin{array}{l}\text { Durch- } \\
\text { schnittliche } \\
\text { Verweil- } \\
\text { dauer }\end{array}$ & $\begin{array}{l}\text { Durch- } \\
\text { schnittliche } \\
\text { Bettenaus- } \\
\text { lastung }\end{array}$ \\
\hline & Anzahl & Anzahl & Anzahl & Anzahl & Tage & Prozent \\
\hline 1991 & 2411 & 665.565 & 83,2 & 13.924 .907 & 14,6 & 83,8 \\
\hline 1992 & 2381 & 646.995 & 80,3 & 14.233 .471 & 13,9 & 83,6 \\
\hline 1994 & 2337 & 618.176 & 75,9 & 14.626 .639 & 12,7 & 82,1 \\
\hline 1995 & 2325 & 609.123 & 74,6 & 15.001 .593 & 12,1 & 81,7 \\
\hline 1996 & 2269 & 593.743 & 72,5 & 15.231 .877 & 11,4 & 80,2 \\
\hline 1997 & 2258 & 580.425 & 70,7 & 15.510 .578 & 11 & 80,7 \\
\hline 1998 & 2263 & 571.629 & 69,7 & 15.952 .070 & 10,7 & 81,9 \\
\hline 1999 & 2252 & 565.268 & 68,9 & 16.260 .785 & 10,4 & 81,8 \\
\hline 2000 & 2242 & 559.651 & 68,1 & 16.486 .672 & 10,1 & 81,5 \\
\hline 2001 & 2240 & 552.680 & 67 & 16.583 .906 & 9,8 & 80,7 \\
\hline 2002 & 2221 & 547.284 & 66,4 & 17.432 .272 & 9,2 & 80,1 \\
\hline 2003 & 2197 & 541.901 & 66,7 & 17.295 .910 & 8,9 & 77,6 \\
\hline
\end{tabular}

Quelle: Statistisches Bundesamt (2006)

Die Kompetenzen für die Krankenhausplanung und -finanzierung sind auf verschiedene Entscheidungsträger verteilt. ${ }^{318}$ Der Bund erlässt die Rahmenbedingungen zur Steuerung der Angebotskapazitäten und zur Vergütung der Krankenhausleistungen. Die Planung der Kapazitäten fällt in die Zuständigkeit der Länder, die den Auftrag haben, die bedarfsgerechte Versorgung der Bevölkerung sicherzustellen. Die Landesbehörden sind verpflichtet, mit den im Land beteiligten Verbänden und Institutionen eng zusammenzuarbeiten, wobei auch das betroffene Krankenhaus über ein Anhörungsrecht verfügt. Diese Beteiligungsrechte umfassen jedoch nur Anhörung und Beratung, so dass die Entscheidungsbefugnis über den Krankenhausplan letztlich beim jeweiligen Bundesland liegt. Die Krankenkassen können mit den Krankenhäusern zwar eine geringere Bettenzahl vereinbaren als es der Plan vorsieht, aber die Leistungsstruktur des Krankenhauses darf nicht geändert werden. ${ }^{319}$ Ein Krankenhaus, das

${ }^{317} \mathrm{Vgl}$. Allgemeine Ortskrankenkasse (AOK) (2005e).

${ }^{318} \mathrm{Vgl}$. im Folgenden Rosenbrock/Gerlinger (2004), S. $143 \mathrm{ff}$.

${ }^{319} \mathrm{Vgl}$. 109 SGB V. 
in den Landeskrankenhausplan aufgenommen wurde, besitzt einen Rechtsanspruch auf den Abschluss eines Versorgungsvertrags mit den zuständigen Krankenkassenverbänden. Unter bestimmten restriktiven Bedingungen können die Verbände diesen Vertrag kündigen, de facto besteht jedoch für die Kassen ein Kontrahierungszwang mit den Planungskrankenhäusern. Damit bleibt den Krankenkassen kaum Entscheidungsfreiheit beim Vertragsabschluss mit den jeweiligen Krankenhäusern. Die Planung obliegt weitestgehend den Ländern, die die Möglichkeit haben, neben den gesundheitspolitischen auch andere Ziele zu verfolgen. Aus ökonomischer Sicht führt dies zu Problemen, da die Finanzierung der Vertragskrankenhäuser getrennt durch zwei voneinander unabhängige Entscheidungsträger erfolgt. Bei dieser dualen Finanzierung trägt der Staat die Investitionskosten der Krankenhäuser, also die Kosten, die durch die Vorhaltung von Krankenhauskapazitäten entstehen, während die Finanzierung der laufenden Kosten durch die Krankenkassen erfolgt, die seit 2004 mittels DRGs mit den Krankenhäusern abrechnen. Der Beitrag des Staates kann ökonomisch begründet werden mit der Theorie der „option demand“, die erstmals im Jahre 1964 von B. A. Weisbrod diskutiert wurde: ${ }^{320}$ Das Vorhandensein von freien Betten in Krankenhäuser kann auch gesunden Menschen einen Nutzen stiften, da auf diese Weise ihre Versorgung im eventuellen Krankheitsfall sichergestellt ist (sog. Optionskonsum). Allerdings ist der Konsum dieser Option nicht rival, d.h. die Bereitstellung eines Bettes bietet mehreren Menschen Versorgungssicherheit. Zudem ist ein Ausschluss von Nichtzahlern im Krankheitsfall aus ethischen Gründen kaum möglich, so dass ein Anreiz zum Freifahrerverhalten besteht. Um die Finanzierung des öffentlichen Gutes „Bettenkapazität“ zu sichern, kann daher eine Finanzierung aus Steuermitteln sinnvoll sein. ${ }^{321}$ Die getrennt Finanzierung von Investitions- und laufenden Kosten führt jedoch dazu, dass die Länder bei der Krankenhausplanung die Folgekosten ihrer Entscheidung nicht berücksichtigen müssen, während die Krankenkassen als Träger der laufenden Kosten wiederum nicht in die Investitionsentscheidungen einbezogen werden. Infolgedessen werden eher zu hohe Kapazitäten aufgebaut. Zu beachten ist aber auch, dass die Krankenhausfinanzierung von der jeweiligen finanziellen Situation des zuständigen Bundeslandes abhängig ist, so das letztlich davon auszugehen ist, dass die Investitionsentscheidungen weniger einer langfristigen Strategie folgen als vielmehr auf Basis der jeweiligen Kassenlage getroffen werden. Es ist daher unwahrscheinlich, dass auf diesem Weg die tatsächlich notwendigen Kapazitäten bereitgestellt werden.

Die „Kohll/Decker“-Rechtsprechung des EuGH nimmt auf die deutschen Krankenhäuser weniger Einfluss als auf den ambulanten Sektor. Der Gerichtshof hatte bereits im Urteil zur Rechtssache „Smits und Peerbooms“ festgestellt, dass ein System der vorherigen Genehmigung notwendig sei, um im Inland ein ausgewogenes Angebot qualitativ hochwertiger Krankenhausversorgung zu gewährleisten, das ständig in ausreichendem Maß zugänglich ist. Zudem trage ein solches System zur Beherrschung der Kosten bei und verhindere eine Verschwendung finanzieller, technischer und personeller

${ }^{320} \mathrm{Vgl}$. Weisbrod (1964).

${ }^{321} \mathrm{Vgl}$. Weisbrod (1964), S. 471-477; Cullis/West (1979), S. 35; Breyer/Zweifel/Kifmann (2005), S. 179. 
Ressourcen. ${ }^{322}$ Dies rechtfertigt eine solche Beschränkung der Dienstleistungsfreiheit nach europäischem Recht. Diese Auffassung bekräftigte der EuGH nochmals in seinem Urteil zur Rechtssache „Müller-Fauré und van Riet“. ${ }^{323}$ Demnach müssen die Krankenkassen nur dann die Kosten einer geplanten Inanspruchnahme von Krankenhausleistungen im Ausland übernehmen, wenn sie zuvor eine Genehmigung dafür erteilt haben. Dieses System der vorherigen behördlichen Genehmigung muss auf objektiven und nicht-diskriminierenden Kriterien beruhen, die im Voraus bekannt sind und sich auf eine leicht zugängliche Verfahrensregelung stützten. Zudem muss es dazu geeignet sein, den Betroffenen zu garantieren, dass über ihren Antrag innerhalb angemessener Frist objektiv und unparteilich entschieden wird. ${ }^{324}$ Die Patienten haben einen Rechtsanspruch auf die Erteilung einer Genehmigung, wenn die vom EuGH formulierten Bedingungen erfüllt sind. ${ }^{325}$ Die Genehmigung darf darüber hinaus nicht aus rein wirtschaftlichen Erwägungen abgelehnt werden. ${ }^{326}$ Das Bestehen einer Warteliste für eine Behandlung kann darum die Ablehnung der Genehmigung nicht rechtfertigen, vielmehr schränkt eine zu lange oder unübliche Wartezeit den Zugang zu einer ausgewogenen und qualitativ hochwertigen Krankenversorgung ein und ist darum als Grund für eine Beschränkung des Zugangs zu ausländischen Leistungen nicht europarechtskonform. Wartelisten, welche die konkreten Umstände des Gesundheitszustands des Patienten nicht berücksichtigen, können demnach nicht als Begründung für die Ablehnung einer Genehmigung herangezogen werden, sondern sprechen eher für einen Anspruch der Patienten auf eine Erlaubnis zur stationären Behandlung im Ausland. ${ }^{327}$ Die nationalen Behörden müssen gemäß des EuGH-Urteils deshalb bei der Aufstellung der Wartelisten die konkreten Umstände des Gesundheitszustands berücksichtigen und gegebenenfalls eine Genehmigung zur stationären Auslandsbehandlung erteilen, wenn die Wartezeit „zu lange oder unüblich ${ }^{6328}$ ist. $\mathrm{Da}$ in der stationären Versorgung in Deutschland Wartelisten kaum existieren, ist zu erwarten, dass dieser Aspekt der EuGH-Rechtsprechung zu keiner signifikante Abwanderung von Patienten führen wird. Vielmehr könnten deutsche Kliniken vom international guten Ruf des deutschen Gesundheitswesens und der Existenz von Wartelisten in anderen europäischen Ländern profitieren.

Auch ein weiterer Aspekt der EuGH-Rechtsprechung zur Dienstleistungsfreiheit begünstigt eine Zunahme der Zahl von Behandlungen ausländischer Patienten in Deutschland. In der Rechtssache „Smits und Peerbooms“ hatte der EuGH u.a. darüber

\footnotetext{
${ }^{322} \mathrm{Vgl}$. Europäischer Gerichtshof (2001a), Rn. 78f.

${ }^{323} \mathrm{Vgl}$. Europäischer Gerichtshof (2003e), Rn. 79f.

${ }^{324} \mathrm{Vgl}$. Europäischer Gerichtshof (2003e), Rn. 85.

${ }^{325} \mathrm{Vgl}$. dazu Abschnitt 2.3.3.1. Eine Genehmigung darf nur dann versagt werden, wenn der Patient die gleiche oder eine ebenso wirksame Behandlung rechtzeitig in einer vertraglich gebundenen Einrichtung im Inland erhalten kann, wobei nicht nur der Gesundheitszustand des Patienten, das Ausmaß der Schmerzen oder die Art der Behinderung, sondern auch die Vorgeschichte des Patienten zu berücksichtigen ist. Vgl. Europäischer Gerichtshof (2003e), Rn. 90.

${ }^{326} \mathrm{Vgl}$. Europäischer Gerichtshof (2003e), Rn. 92.

${ }^{327}$ Diese Auffassung bestätigte der EuGH im Jahre 2006 nochmals in seinem Urteil in der Rechtssache „Watts“. Vgl. Europäischer Gerichtshof (2006), Rn. 119.

${ }^{328} \mathrm{Vgl}$. Europäischer Gerichtshof (2003e), Rn. 92.
} 
zu entscheiden, ob die Genehmigung zur Behandlung im Ausland von der Bedingung abhängig gemacht werden darf, dass sie in ärztlichen Kreisen üblich ist. Der Gerichtshof urteilte, dass eine solche Einschränkung mit dem Gemeinschaftsrecht vereinbar sei, sofern sich die Üblichkeit der Behandlung nicht auf nationale Standards bezieht, sondern darauf, „was von der internationalen Medizin als hinreichend erprobt und anerkannt angesehen wird“" ${ }^{329}$ Grundsätzlich kann sich eine solche Regelung positiv auf die Inanspruchnahme von Kliniken auswirken, die über eine gute technologische Ausstattung verfügen und auf Basis der aktuellen wissenschaftlichen Erkenntnisse arbeiten. Ein guter internationaler Ruf der deutschen Versorgung führt daher auch auf diesem Weg tendenziell zu einer Zuwanderung ausländischer Patienten. Studien zeigen, dass die Zahl der ausländischen Patienten, die sich in deutschen Krankenhäusern behandeln lassen, zunimmt. Grundsätzlich sind für die deutschen Kliniken Behandlungen ausländischer Patienten wirtschaftlich interessant, da sie außerhalb der geltenden deutschen Budgets abgerechnet werden, jedoch ist der Umfang dieser Inanspruchnahme noch so gering, dass bisher keine Auswirkungen auf die finanzielle Ausstattung des gesamten stationären Systems festzustellen sind. ${ }^{330}$ Zusammenfassend ist daher festzustellen, dass die Urteile des EuGH zur Gültigkeit des Waren- und Dienstleistungsverkehrs im stationären Sektor wenig Einfluss auf die bestehenden Regelungen genommen haben. Eine zunehmende Mobilität der ausländischen Patienten würde sich auf die deutsche Krankenhauslandschaft aber eher positiv auswirken. Allerdings gilt zu beachten, dass die duale Finanzierung der Krankenhauskosten dazu führt, dass bei einer Behandlung ausländischer Patienten lediglich die Leistungsausgaben und nicht die entstehenden Investitionskosten mit dem ausländischen Finanzierungsträger abgerechnet werden können, während sich im umgekehrten Fall die deutschen Krankenkassen in Ländern mit monistischer Krankenhausfinanzierung an den Investitionskosten beteiligen. ${ }^{331}$ Hier würde der Übergang von der dualen zur monistischen Finanzierung zu einer gerechteren Zurechnung der Kosten führen und zudem die Vergleichbarkeit der Behandlungsausgaben erhöhen.

Die Erwartung größerer struktureller Änderungen im stationären Sektor wurde im Jahre 2003 mit dem Urteil des EuGH in der Rechtssache „Jaeger" verbunden. ${ }^{332} \mathrm{Der}$ EuGH hatte in dieser Rechtssache die Frage zu beantworten, ob verschiedene Regelungen des deutschen Arbeitszeitgesetzes (ArbZG) vom 6. Juni 1994 mit den Vorschriften der Arbeitszeitrichtlinie 93/104/EG ${ }^{333}$ vereinbar waren. Das deutsche $\mathrm{Ar}$ beitsrecht unterscheidet zwischen „Arbeitsbereitschaft“, „Bereitschaftsdienst“ und „Rufbereitschaft““ ${ }^{334}$ Arbeitsbereitschaft liegt vor, wenn der Arbeitnehmer dem Arbeitgeber

\footnotetext{
${ }^{329} \mathrm{Vgl}$. Europäischer Gerichtshof (2001 a), Rn. 94.

${ }^{330} \mathrm{Vgl}$. Juszczakm/Zangerle (2004), S. 1148.

${ }^{331} \mathrm{Vgl}$. Schulte (2000), S. 35.

${ }^{332} \mathrm{Vgl}$. Europäischer Gerichtshof (2003c).

${ }^{333}$ Diese Richtlinie wurde auf Basis des Art. 118a EGV (alt) erlassen, der dem Rat auferlegte, die Verbesserung der Arbeitsumwelt zu fördern, um die Sicherheit und die Gesundheit der Arbeitnehmer verstärkt zu schützen. Vgl. Erwägungsgründe zur Richtlinie 93/104/EG. Sie wurde durch die Richtlinie 2003/88/EG (sog. Arbeitszeitrichtlinie der EU) ersetzt.

${ }^{334} \mathrm{Vgl}$. auch im Folgenden Europäischer Gerichtshof (2003c), Rn. 12 -16.
} 
am Arbeitsplatz zur Verfügung stehen und sich ständig bereithalten muss, um im Bedarfsfall von sich aus tätig werden zu können. Während des Bereitschaftsdienstes ist der Arbeitnehmer dazu verpflichtet, sich innerhalb oder außerhalb des Betriebes an einem vom Arbeitgeber bestimmten Ort aufzuhalten und sich auf Anforderung des Arbeitgebers zur Arbeitsaufnahme bereitzuhalten. Er darf jedoch ruhen oder sich anderweitig beschäftigen, solange seine beruflichen Leistungen nicht erforderlich sind. Zudem definiert das deutsche Arbeitszeitgesetz noch die Rufbereitschaft, die dadurch gekennzeichnet ist, dass der Arbeitnehmer sich nicht an einer vom Arbeitgeber bestimmten Stelle bereithalten muss, sondern nur jederzeit erreichbar sein muss, um seine beruflichen Aufgaben auf Abruf unverzüglich wahrnehmen zu können. Das deutsche Arbeitszeitgesetz von 1994 sah vor, dass lediglich die Arbeitsbereitschaft in vollem Umfang Arbeitszeit darstellt. Bereitschaftsdienst und Rufbereitschaft wurden dagegen als Ruhezeiten behandelt, mit Ausnahme des Teils der Dienstzeit, in dem der Arbeitnehmer tatsächlich seine beruflichen Aufgaben wahrgenommen hat. In seinem Urteil entschied der Gerichtshof nun, dass der Bereitschaftsdienst, den ein Arzt im Krankenhaus leistet, gemäß Richtlinie 93/104/EG in vollem Umfang als Arbeitszeit zu werten sei. Schätzungen zufolge hätte die Umsetzung dieses Urteils es notwendig gemacht, in Deutschland eine bedeutende Zahl neuer Stellen für Klinikärzte zu schaffen. Der Marburger Bund ging von 15.000 neuen Stellen aus und die Deutsche Krankenhausgesellschaft (DKG) erwartete sogar, dass 27.000 Stellen neu zu schaffen seien. ${ }^{335}$ Das deutsche Arbeitszeitgesetz wurde schließlich zum 1.1.2004 dahingehend geändert, dass nun auch der Bereitschaftsdienst als Arbeitszeit gewertet wurde. Allerdings galt für bestehende Tarifverträge eine Übergangszeit bis Ende 2005. Zudem wurde gleichzeitig eine sog. „Opt-Out"-Regelung in das deutsche Arbeitszeitgesetz aufgenommen, die entsprechend der EU-Arbeitszeitrichtlinie von den Mitgliedstaaten in deren nationaler Gesetzgebung berücksichtigt werden kann. ${ }^{336}$ Sie erlaubt es, im Rahmen eines Tarifvertrags die Arbeitszeit ohne einen späteren Zeitausgleich über die vorgesehene maximale Arbeitszeit von zehn Stunden je Werktag ${ }^{337}$ hinaus zu verlängern, wenn in die Arbeitszeit regelmäßig und in erheblichem Umfang Arbeitsbereitschaft oder Bereitschaftsdienst fallen und sichergestellt ist, dass die Gesundheit des Arbeitnehmers nicht gefährdet ist. Eine solche Arbeitszeitverlängerung ist aber nur möglich, wenn der Beschäftigte ihr schriftlich zustimmt. ${ }^{338}$ Dieses Opt-out war zuvor im deutschen ArbZG nicht vorgesehen und bietet nun die Möglichkeit, die Folgen der EuGH-Rechtsprechung abzumildern, so dass die Zahl der neu zu schaffenden Stellen deutlich unter den oben genannten Schätzungen bleiben dürfte.

Die Europäische Union legte nach dem „Jaeger"-Urteil am 22.9.2004 und am 31.05.2005 Vorschläge zur Änderung der Richtlinie 2003/88/EG vor ${ }^{339}$, die u.a. die

\footnotetext{
${ }^{335} \mathrm{Vgl}$. Hackenbroch (2003), S. 166.

${ }^{336} \mathrm{Vgl}$. Art. 22 der Richtlinie 2003/88/EG.

${ }^{337}$ „Die werktägliche Arbeitszeit der Nachtarbeitnehmer darf acht Stunden nicht uberschreiten. Sie kann auf bis zu zehn Stunden nur verlängert werden, wenn abweichend von $\S 3$ innerhalb von einem Kalendermonat oder innerhalb von vier Wochen im Durchschnitt acht Stunden werktäglich nicht überschritten werden." $§ 6$ Abs. 2 ArbZG.

${ }^{338} \mathrm{Vgl}$. Spielberg (2004), S. 3148.

${ }^{339} \mathrm{Vgl}$. Europäische Kommission (2004d) und Europäische Kommission (2004a).
} 
Einführung neuer Definitionen zu den Begriffen „Bereitschaftsdienst" und „inaktive Zeit während des Bereitschaftsdienstes" vorsehen. Der Begriff des Bereitschaftsdiensts ist in der derzeit gültigen EU-Arbeitszeitrichtlinie nicht enthalten. Die Vorschläge der Kommission sehen vor, ihn zu definieren als „Zeit, in der der Arbeitnehmer an seinem Arbeitsplatz zur Verfügung stehen muss, um auf Aufforderung des Arbeitgebers seine Tätigkeit ausüben oder seine Aufgaben wahrnehmen zu können“340, während als ,inaktive Zeit" diejenige Zeit abgrenzt wird, ,in der der Arbeitnehmer Bereitschaftsdienst [...] hat, aber von seinem Arbeitgeber nicht zur Ausübung seiner Tätigkeit oder Wahrnehmung seiner Aufgaben aufgefordert wird. ${ }^{\text {(341 }}$ Die inaktive Zeit während des Bereitschaftsdiensts soll dann nicht als Arbeitszeit gewertet werden, es sei denn nationalstaatliche Regelungen ${ }^{342}$ sehen etwas anderes vor. Die Zeit, in der der Arbeitnehmer während des Bereitschaftsdiensts dagegen effektiv tätig ist, wird immer zur Arbeitszeit gerechnet. ${ }^{343}$ Diese Änderung der Arbeitszeitrichtlinie soll dazu dienen, die Auswirkungen der durch das EuGH-Urteil notwendigen Novellierungen der Arbeitszeitgesetze abzumildern.

Daneben sehen die Änderungsvorschläge eine Einschränkung der Opt-out-Regelungen vor. ${ }^{344}$ Der Anlass hierfür sind Erfahrungen in Großbritannien, das als einziger Mitgliedstaat diese Möglichkeit bereits vor dem „Jaeger“-Urteil in seiner nationalen Gesetzgebung vorsah. Die Kommission hat in einem Bericht, der u.a. die Ausgestaltung und Anwendung dieser Opt-out-Regelung in Großbritannien untersuchte, festgestellt, dass die dortige „Gesetzgebung und Praxis nicht alle von der Richtlinie vorgesehenen Garantien zu bieten scheinen “345. Auch die verfügbaren Daten zur praktischen Anwendung der Regelungen sprechen dafür, dass das britische System der Arbeitszeitgestaltung trotz der Einführung der europäischen Arbeitszeitrichtlinie im Jahre 1994 hauptsächlich aufgrund der Anwendung des Opt-out unverändert blieb und somit das Ziel der Richtlinie verfehlt wurde. ${ }^{346}$ Vorgeschlagen ist eine Änderung der entsprechenden Regelung dahingehend, dass die notwendige Zustimmungserklärung des Arbeitnehmers nicht mehr gemeinsam mit dem Arbeitsvertrag oder während der Probezeit unterschrieben werden darf und dass die Zustimmung auf ein Jahr befristet wird - mit der Möglichkeit, sie danach zu verlängern. Bei Anwendung der Opt-out-Klausel soll eine Beschränkung der maximalen Wochenarbeitszeit auf 55 Stunden erfolgen, wobei von dieser Regelung durch Tarifvereinbarungen abgewichen werden kann.

Zum derzeitigen Zeitpunkt zeichnet sich keine schnelle Einigung über die Änderung der Richtlinie ab. Beim Treffen des europäischen „Beschäftigungsrates“ am 2. Juni

\footnotetext{
${ }^{340}$ Europäische Kommission (2004d), S. 9.

${ }^{341} \mathrm{Vgl}$. Europäische Kommission (2004d), S. 9.

${ }^{342}$ Solche nationalstaatlichen Regelungen können Gesetze, tarifvertragliche Vereinbarungen oder Vereinbarungen zwischen den Sozialpartnern in Übereinstimmung mit der einzelstaatlichen Gesetzgebung sein.

${ }^{343} \mathrm{Vgl}$. Europäische Kommission (2004d), S. 9.

${ }^{344} \mathrm{Vgl}$. Europäische Kommission (2004d), S. S. $11 \mathrm{f}$.

${ }^{345}$ Europäische Kommission (2003c), S. 10, z.B. erfolgte die Unterzeichnung dort mit dem Arbeitsvertrag und die vorgeschrieben Listenführung war inadäquat.

${ }^{346} \mathrm{Vgl}$. Europäische Kommission (2003c), S. 12
} 
2005 konnte keine Einigung erzielt werden, wobei die dargestellte Änderung der Optout-Klausel den Hauptstreitpunkt darstellte. Eine Gruppe von Mitgliedstaaten - angeführt von Großbritannien und Polen - lehnte den am 31. Mai durch die Kommission vorgelegten Kompromissvorschlag ${ }^{347}$ ab. Es ist unklar, wann und ob die geplanten Änderungen umgesetzt werden können. Damit gilt bis auf weiteres in Deutschland das oben erwähnte Arbeitszeitgesetz vom 1.1.2004, das den Bereitschaftsdienst vollständig als Arbeitszeit wertet, aber mit Zustimmung des Beschäftigten eine Überschreitung der maximalen Wochenarbeitszeit von 48 Stunden zulässt. Auf diese Weise besteht für die Krankenhäuser die Möglichkeit, die nach dem „Jaeger“-Urteil des EuGH erwarteten dramatische Auswirkungen auf die Stellensituation im stationären Sektor nach Ablauf der Übergangsfrist zum 1.1.2006 durch eine Nutzung der Opt-out-Klausel abzuschwächen, so dass sich auch auf diesem Weg der Einfluss der EU im stationären Sektor nur sehr bescheiden ausnimmt.

\subsubsection{Vorsorge- und Rehabilitationseinrichtungen}

Neben den Krankenhäusern gehören zum stationären Sektor die Vorsorge- und Rehabilitationseinrichtungen, die die stationäre und Teile der ambulanten Versorgung der Patienten im Bereich der Vor- und Nachsorge übernehmen. Tabelle 2 zeigt die Entwicklung der Vorsorge- und Rehabilitationseinrichtungen von 1991 bis 2005.

Die Jahre 1991 bis 1996 waren von einer Entwicklung geprägt, die mit einer steigenden Zahl von Häusern und aufgestellten Betten sowie einer durchschnittlichen Bettenauslastung von deutlich über 80 Prozent einherging. Die Gesundheitsreform im Jahre 1996 brachte dann starke Einschnitte mit sich, die zu einer spürbaren Reduktion der Fallzahlen und Pflegetage sowie der geringsten Bettenauslastung im betrachteten Zeitraum führten. Nach zahlreichen Stellenstreichungen und drohenden Schließungen einer signifikanten Zahl von Einrichtungen wurden von Seiten der Politik zusätzliche finanzielle Mittel zur Verfügung gestellt, ${ }^{348}$ so dass die Jahre 1997 bis 2001 für die Einrichtungen hinsichtlich der Fallzahlen und der Bettenauslastung eher positiv verliefen. In den Jahren 2002 bis 2005 sanken wiederum die Fallzahlen und Pflegetage sowie die Bettenauslastung; die Zahl der Einrichtungen insgesamt ging weiter zurück.

Rund zwei Drittel der deutschen Vorsorge- und Rehabilitationskliniken mit insgesamt annähernd 150.000 Betten befinden sich in Heilbädern und Kurorten. ${ }^{349}$ Diese Orte liegen in der Regel abseits von großen Ballungsgebieten in eher ländlichen und peripheren Gegenden und sind oftmals von großer Bedeutung für die jeweilige Region, beispielsweise in Bezug auf das kulturelle Angebot oder die Verkehrsinfrastruktur. Die Kureinrichtungen zählen häufig zu den wichtigsten Arbeitgebern in diesen Regionen, so dass eine große wirtschaftliche Abhängigkeit von der Gesamtentwicklung des deutschen Kurwesens besteht. ${ }^{350}$

\footnotetext{
${ }^{347} \mathrm{Vgl}$. Europäische Kommission (2004a).

${ }^{348}$ Vgl. Clade (1997), S. A 2178.

${ }^{349}$ Vgl. Deutscher Heilbäderverband (DHV) (2005).

${ }^{350} \mathrm{Vgl}$. Deutscher Heilbäderverband (DHV) (2005).
} 
Tabelle 2: Einrichtungen, Betten und Patientenbewegung in Vorsorge- und Rehabilitationseinrichtungen, $1991-2005$

\begin{tabular}{|l|l|l|l|l|l|l|}
\hline \multirow{3}{*}{} & \multicolumn{3}{|l|}{$\begin{array}{l}\text { Vorsorge- oder Reha- } \\
\text { bilitationseinrichtungen }\end{array}$} & \multicolumn{4}{l|}{ Patientenbewegung } \\
\cline { 2 - 7 } insgesamt & $\begin{array}{l}\text { aufgestellte } \\
\text { Betten }\end{array}$ & Fallzahl & Pflegetage & $\begin{array}{l}\text { durch- } \\
\text { schnittliche } \\
\text { Verweildauer }\end{array}$ & $\begin{array}{l}\text { durch- } \\
\text { schnittliche } \\
\text { Bettenaus- } \\
\text { lastung }\end{array}$ \\
\cline { 2 - 7 } & Anzahl & & Anzahl & in 1.000 & in Tagen & in Prozent \\
\hline 1991 & 1.181 & 144.172 & 1.473 .427 & 45.729 & 31,0 & 86,9 \\
\hline 1992 & 1.209 & 149.910 & 1.574 .891 & 48.833 & 31,0 & 89,0 \\
\hline 1993 & 1.245 & 155.631 & 1.632 .218 & 50.469 & 30,9 & 88,8 \\
\hline 1994 & 1.329 & 172.675 & 1.764 .518 & 55.069 & 31,2 & 87,4 \\
\hline 1995 & 1.373 & 181.633 & 1.895 .887 & 58.820 & 31,0 & 88,7 \\
\hline 1996 & 1.404 & 189.888 & 1.916 .531 & 57.839 & 30,2 & 83,2 \\
\hline 1997 & 1.387 & 188.869 & 1.575 .454 & 42.972 & 27,3 & 62,3 \\
\hline 1998 & 1.395 & 190.967 & 1.746 .345 & 46.107 & 26,4 & 66,1 \\
\hline 1999 & 1.398 & 189.597 & 1.915 .334 & 49.874 & 26,0 & 72,1 \\
\hline 2000 & 1.393 & 189.822 & 2.046 .227 & 52.852 & 25,8 & 76,1 \\
\hline 2001 & 1.388 & 189.253 & 2.096 .904 & 53.514 & 25,5 & 77,5 \\
\hline 2002 & 1.343 & 184.635 & 2.041 .272 & 52.107 & 25,5 & 77,3 \\
\hline 2003 & 1.316 & 179.789 & 1.899 .558 & 49.204 & 25,9 & 75,0 \\
\hline 2004 & 1.294 & 176.473 & 1.889 .362 & 47.442 & 25,1 & 73,5 \\
\hline $2005^{1)}$ & 1270 & 174.521 & 1.815 .366 & 46.787 & 25,8 & 73,4 \\
\hline
\end{tabular}

1) vorlăufiges Ergebnis

Quelle: Statistisches Bundesamt (2006); eigene Darstellung

In Deutschland existieren für medizinische Vorsorge- und Rehabilitationsleistungen verschiedene Finanzierungsträger. Die wichtigsten sind die gesetzlichen Krankenkassen sowie die Rentenversicherungsträger, wobei die gesetzlichen Krankenkassen vor allem die Genehmigung und Finanzierung der Vorsorgeleistungen übernehmen, während für Rehabilitationsleistungen zahlenmäßig am häufigsten Träger der gesetzlichen Rentenversicherung zuständig sind. Die Anreize und Möglichkeiten der Patienten zur Inanspruchnahme von Kuren im Ausland ergeben sich vor allem durch die jeweiligen Regelungen im Sozialgesetzbuch und die anzuwendenden europarechtlichen Regelungen. Unabhängig davon, welcher Träger verantwortlich ist, müssen alle ambulanten und stationären Vorsorge- und Rehabilitationsleistungen vor ihrem Beginn vom Arzt 
verordnet und beim zuständigen Kostenträger beantragt werden. Für die Inanspruchnahme von Kuren ${ }^{351}$ ist also generell eine Genehmigung erforderlich - auch wenn sie in Deutschland in Anspruch genommen werden.

Übernimmt die Krankenkasse die Kosten für medizinische Vorsorgeleistungen, so bestimmt sie Art, Dauer, Umfang, Beginn und Durchführung der Leistung sowie die Vorsorgeeinrichtung, in der die Maßnahme durchgeführt wird. ${ }^{352}$ Die Kasse erstattet die Kosten für An- und Abreise, Heil-, Hilfs- und Arzneimittel, Unterbringung und die ärztlichen Honorare. Die Patienten haben eine Zuzahlung von $10 €$ pro Tag zu tragen. Bei ambulanten Leistungen entfällt die Übernahme der Verpflegungs-, Unterbringungs- und Reisekosten; die Krankenkassen können hierfür jedoch einen Zuschuss von bis zu $13 €$ pro Tag zahlen. ${ }^{353}$ Für die in Anspruch genommenen Kurmittel (z.B. Bäder und Massagen) fallen die gesetzlich vorgeschriebenen Zuzahlungen an. Der Patient kann eine Kureinrichtung - in Abhängigkeit von der jeweiligen Indikation - im Einvernehmen mit seinem Arzt auswählen.

Die gesetzliche Grundlage für eine Kostenübernahme für medizinische Rehabilitationsleistungen durch andere Leistungsträger als die Krankenversicherung bilden die Regelungen des SGB IX. Der zuständige Rentenversicherungsträger trägt die Kosten für ärztliche Betreuung, therapeutische Leistungen und medizinische Anwendungen ${ }^{354}$, Reise, Unterkunft und Verpflegung, ${ }^{355}$ soweit sie für die Teilnahme an der Rehabilitation notwendig sind. Die Zuzahlungen betragen auch hier $10 €$ pro Tag. Ambulante Reha-Maßnahmen werden wohnortnah entweder in stationären oder in eigenständigen ambulanten Zentren durchgeführt.

Tabelle 3 gibt einen Überblick über die verschiedenen Vorsorge- und Rehabilitationsleistungen, deren Zielsetzung sowie die Einrichtungen, in denen die jeweiligen Leistungen erbracht werden.

Hinsichtlich der Kostenübernahme für Kuren im Ausland durch die verschiedenen deutschen Leistungsträger bestehen divergierende Regelungen. Eine Inanspruchnahme von Eltern-Kind-Kuren ist im Ausland nicht möglich, da derartige Leistungen in keinem anderen Mitgliedstaat Bestandteil des Leistungskatalogs darstellen und darum keine geeigneten Einrichtungen vorhanden sind. ${ }^{356}$ Eine Inanspruchnahme von Rehabilitationsleistungen im Ausland ist dahingehend mit Schwierigkeiten verbunden, dass diese Leistungen dort oftmals anders ausgerichtet sind als in Deutschland. Zudem ist im Ausland keine Einbeziehung des sozialen und beruflichen Umfeldes möglich und ein nahtloser Übergang in die Nachsorge schwieriger. Nichtsdestotrotz kann in Einzel-

\footnotetext{
${ }^{351}$ Der Begriff „Kur“ findet sich seit dem GKV-Modernisierungsgesetz vom 1.1.2000 nicht mehr im Sozialgesetzbuch, das seitdem stattdessen von Vorsorge- und Rehabilitationsleistungen spricht. Er wird im vorliegenden Text jedoch als Sammelbegriff für die entsprechenden Leistungen verwendet.

${ }^{332}$ Vgl. § 23 Abs. 5 SGB V.

${ }^{353}$ Bei Kleinkindern beträgt der Zuschuss bis zu $21 €$ pro Tag. Vgl. § 23 Abs. 2 SGB V.

${ }^{354} \mathrm{Vgl}$. § 26 Abs. 2 SGB IX.

${ }^{355}$ Vgl. § 53 SGB XI.

${ }^{356} \mathrm{Vgl}$. auch im Folgenden Spitzenverbände der Krankenkassen (2005), S. $3 \mathrm{f}$.
} 
fällen eine solche Durchführung (z.B. im Heimatland eines Patienten mit ausländischer Herkunft) sinnvoll sein.

Tabelle 3: Übersicht über Vorsorge- und Rehabilitationsleistungen

\begin{tabular}{|c|c|c|}
\hline & Ziel & Einrichtung \\
\hline $\begin{array}{l}\text { ambulante Vorsorge- } \\
\text { leistung }\end{array}$ & \multirow{2}{*}{$\begin{array}{l}\text { Behebung einer Schwächung } \\
\text { der Gesundheit, Verhütung } \\
\text { einer Krankheit oder Ver- } \\
\text { meidung ihrer Verschlimme- } \\
\text { rung }\end{array}$} & $\begin{array}{l}\text { staatlich anerkannte Heil- } \\
\text { bäder, private Unterbrin- } \\
\text { gung }\end{array}$ \\
\hline $\begin{array}{l}\text { stationäre Vorsorge- } \\
\text { leistung }\end{array}$ & & $\begin{array}{l}\text { staatlich anerkannte Heil- } \\
\text { bäder, Unterbringung in } \\
\text { einer Kurklinik }\end{array}$ \\
\hline $\begin{array}{l}\text { ambulante Rehabilita- } \\
\text { tion }\end{array}$ & \multirow{2}{*}{$\begin{array}{l}\text { Beseitigung oder Milderung } \\
\text { einer bestehenden Krankheit } \\
\text { oder Behinderung }\end{array}$} & wohnortnahe Einrichtung \\
\hline $\begin{array}{l}\text { stationäre Rehabilita- } \\
\text { tion }\end{array}$ & & $\begin{array}{l}\text { staatlich anerkannte Heil- } \\
\text { bäder, Unterbringung in } \\
\text { einer Klinik }\end{array}$ \\
\hline $\begin{array}{l}\text { ambulante Kompakt- } \\
\text { kur }\end{array}$ & $\begin{array}{l}\text { intensive Therapieprogram- } \\
\text { me in kleinen Gruppen, vor } \\
\text { allem bei chronischen Krank- } \\
\text { heiten }\end{array}$ & $\begin{array}{l}\text { staatlich anerkannte Heil- } \\
\text { bäder, private Unterbrin- } \\
\text { gung, kürzere Dauer (drei } \\
\text { Wochen) }\end{array}$ \\
\hline $\begin{array}{l}\text { Anschlussheil- } \\
\text { behandlung (AHB) } \\
\text { (Rehabilitation) }\end{array}$ & $\begin{array}{l}\text { nach einem stationären Kran- } \\
\text { kenhausaufenthalt bei Er- } \\
\text { krankungen auf der AHB- } \\
\text { Indikationsliste }\end{array}$ & $\begin{array}{l}\text { wohnortnahe Einrichtung } \\
\text { (entweder ambulant oder } \\
\text { stationär) }\end{array}$ \\
\hline $\begin{array}{l}\text { Mutter-/Vater-Kind- } \\
\text { Maßnahmen (Vorsor- } \\
\text { ge und Rehabilitati- } \\
\text { on) } \S 24 \text { SGB V }\end{array}$ & $\begin{array}{l}\text { Vorsorge- und Rehaleistun- } \\
\text { gen, die im Zusammenhang } \\
\text { mit den gesundheitlichen Be- } \\
\text { lastungen durch die Famili- } \\
\text { enarbeit stehen }\end{array}$ & stationäre Unterbringung \\
\hline
\end{tabular}

Quelle: Bundesministerium für Gesundheit und Soziale Sicherheit (BMGS) (2005a); eigene Darstellung

Werden Maßnahmen zur ambulanten oder stationären Vorsorge bzw. Rehabilitation von der gesetzlichen Krankenkasse genehmigt, so können sie grundsätzlich auch in einem anderen EU-Land in Anspruch genommen werden. Wie bei den Leistungen der Akutversorgung existieren auch hier die beiden Verfahren der Kostenübernahme, zum einen durch die Rechtsprechung des EuGH auf Basis der Waren- und Dienstleistungsfreiheit, zum anderen mit einer vorherigen Genehmigung der zuständigen Krankenkasse für eine Auslandsbehandlung auf Grundlage der Verordnung 1408/71/EWG. Von der Wahl des Verfahrensweges hängt die Höhe der Kostenerstattung der Krankenkasse ab. Die entsprechenden Regelungen werden in den folgenden Abschnitten näher erläutert. Welche Option für den Patienten günstiger ist, hängt u.a. vom ausländischen Leis- 
tungskatalog sowie den im Ausland entstehenden Kosten und Zuzahlungen ab. Diese Frage kann deshalb nur für den jeweiligen Einzelfall beantwortet werden. Da für die Übernahme der Kosten einer Kur grundsätzlich die Genehmigung der Krankenkasse erforderlich ist, unabhängig davon, ob die Maßnahme im In- oder Ausland erfolgt, scheidet hier eine nachträgliche Kostenübernahme für selbst beschaffte Leistungen, wie sie bei ambulanten ärztlichen Leistungen möglich ist, aber generell aus.

Bei ambulanten Kuren gilt § 13 Abs. 4 SGB V, so dass der Patient bei der Antragstellung durch seinen Arzt einen Wunschkurort angeben und somit einen Ort in einem anderen EU-Mitgliedstaat wählen kann. Deutschen Einrichtungen darf hier von Seiten der Krankenkasse kein grundsätzlicher Vorzug eingeräumt werden. Es ist lediglich darauf zu achten, dass der Kurort medizinisch sinnvoll gewählt wird, die Qualität der Behandlungen genauso gut wie in Deutschland ist und das Personal im Kurort Deutsch sprechen kann. Eine weitere Genehmigung der Kasse für die Inanspruchnahme im Ausland ist nicht notwendig, so dass auch die in der Verordnung 1408/71/EWG aufgeführten Voraussetzungen ${ }^{357}$ nicht erfüllt sein müssen.

Hinsichtlich der finanziellen Konsequenzen für die Patienten ist von Bedeutung, dass die Krankenkassen die Kosten lediglich bis zur Höhe der in Deutschland geltenden Sätze erstatten. Die Patienten haben die Zuzahlungen entsprechend der in Deutschland gültigen Regelungen zu tragen, d.h. im Fall ambulanter Maßnahmen übernehmen sie die gesetzlich vorgeschriebenen Zuzahlungen, z.B. für die in Anspruch genommenen Heilmittel. Diese betragen 10 Prozent der anfallenden Kosten zuzüglich $10 €$ je Verordnung. Die prozentuale Abhängigkeit der Zuzahlung von den Kosten kann den Patienten einen Anreiz bieten, die entsprechenden Leistungen in Ländern in Anspruch zu nehmen, in denen sie zu geringeren Kosten erbracht werden. Hinzu kommt, dass die Versicherten die Unterbringungs- und Verpflegungskosten deutlich senken können, wenn sie die vor allem zugunsten osteuropäischer Länder bestehenden Preisunterschiede nutzen. Da davon auszugehen ist, dass diese Preise mit den Kosten für medizinische Behandlungen stark positiv korrelieren, werden in der Regel die anfallenden Behandlungskosten die deutschen Sätze unterschreiten. Somit ist das Risiko, dass durch die Behandlung Kosten entstehen, die über die in Deutschland geltenden Erstattungen hinausgehen und von den Patienten selbst getragen werden müssten, relativ gering.

Im Fall stationärer Maßnahmen bestimmt die Krankenkasse die Kureinrichtung nach „pflichtgemäßem Ermessen“" ${ }^{358}$ Auch hier ist die Durchführung der Kur im Ausland grundsätzlich möglich. Allerdings ist aus rechtlicher Sicht nicht eindeutig, ob hierfür

\footnotetext{
${ }^{357}$ Wie in Abschnitt 3.2.1 erläutert, muss gemäß Verordnung 1408/71/EWG die zustăndige Krankenkasse eine Genehmigung zur Auslandsbehandlung erteilen, wenn die betreffende Behandlung zum inländischen Leistungskatalog gehört und der Versicherte in Anbetracht seines derzeitigen Gesundheitszustands und des voraussichtlichen Verlaufs der Krankheit diese Behandlung im Inland nicht in einem Zeitraum erhalten kann, der fur diese Behandlung in seinem Wohnsitzstaat normalerweise erforderlich ist. Im Umkehrschluss mulssen die genannten Voraussetzungen erfullt sein, damit die Kasse eine Genehmigung zur Auslandsbehandlung erteilen kann.
}

${ }^{358} \mathrm{Vgl}$. $\$ 23$ Abs. 4 und $\S 40$ Abs. 3 SGB V. 
eine Genehmigung entsprechend der Verordnung 1408/71/EWG benötigt wird. ${ }^{359}$ In diesem Fall wäre eine Genehmigung der Krankenkasse für eine Inanspruchnahme der Kur im Ausland nur dann zu erteilen, wenn eine entsprechende Behandlung nicht in einer angemessenen Zeit in Deutschland möglich wäre. Kingreen (2006) geht jedoch davon aus, dass in Bezug auf Kureinrichtungen der Genehmigungsvorbehalt nicht wie bei Krankenhausleistungen mit der zur Aufrechterhaltung der Versorgung notwendigen Planbarkeit der Infrastruktur begründbar ist. ${ }^{360}$ Demnach wäre eine Inanspruchnahme dieser Leistungen im Ausland ebenfalls nicht von den genannten Voraussetzungen der Verordnung 1408/71/EWG abhängig. Da die Auswahl der Einrichtung der Kasse überlassen bleibt, ist davon auszugehen, dass sich eine verstärkte Inanspruchnahme ausländischer Anbieter vor allem dann ergeben wird, wenn solche Angebote für die Kassen interessant sind, z.B. weil dabei deutlich geringere Behandlungskosten anfallen oder die Kassen sich dadurch eine Steigerung der Attraktivität ihres Angebots erhoffen, die ihre Wettbewerbsposition verbessert. Immer mehr Kassen schließen daher Verträge mit attraktiven osteuropäischen Einrichtungen ${ }^{361}$ und erhalten auf diese Weise die Möglichkeit, durch die Suche nach preisgünstigen Anbietern ihre Ausgaben für diese Maßnahmen deutlich zu senken. Solche Verträge sind nach § 140e SGB V zwischen deutschen Krankenkassen und ausländischen Leistungsanbietern möglich und existieren bereits sowohl für ambulante als auch stationäre Kuren. Nach Angaben der Hanseatischen Krankenkasse (HEK) liegen die Kosten für stationäre Kuren in Tschechien um etwa 10 Prozent unter denen deutscher Einrichtungen. ${ }^{362}$ Bei der Inanspruchnahme stationärer Vorsorge- oder Rehabilitationsmaßnahmen auf Basis der Grundfreiheiten im Ausland übernimmt die Krankenkasse die Kosten lediglich bis zu der Höhe, wie sie in Deutschland entstanden wären. Der Patient hat darum ein finanzielles Interesse daran, dass die im Ausland anfallenden Behandlungskosten die deutschen Sätze nicht übersteigen, da er ansonsten den Differenzbetrag selbst zahlen muss. Da sich für die Kasse nur dann finanzielle Vorteile aus einer Auslandsbehandlung ergibt, wenn die dort anfallenden Kosten geringer sind als die einer vergleichbaren $\mathrm{Be}$ handlung im Inland, wird sie dies bei ihrer Auswahl berücksichtigen, so dass der Patienten nicht befürchten muss, bei einer Auslandsbehandlung höhere Kosten tragen zu müssen als im Inland. Ein finanzieller Vorteil aus geringeren Kosten ergibt sich für die Patienten aber nicht, da die Zuzahlungen für die Patienten wie in Deutschland $10 €$ pro Tag betragen. Die Vorteile der Versicherten aus stationären Maßnahmen im Ausland können sich darum vorrangig nur aus der Qualität der erbrachten Leistungen und der Attraktivität des jeweiligen Kurortes ergeben.

Sowohl bei ambulanten als auch bei stationären Maßnahmen setzt eine Kostenübernahme voraus, dass die in Anspruch genommenen Leistungserbringer im jeweiligen

\footnotetext{
${ }^{359} \mathrm{Vgl}$. auch im Folgenden Kingreen (2006), S. $211 \mathrm{ff}$.

${ }^{360}$ Für eine ausfuhrlichere Darstellung der vom EuGH genannten Rechtfertigungsgründe für einen Genehmigungsvorbehalt bei krankenhausbasierten Leistungen siehe Abschnitt 2.3.3.1, der die entsprechenden EuGH-Urteile erläutert.

${ }^{361}$ Im Jahre 2005 hatten bereits die Hanseatische Krankenkasse (HEK) sowie zwölf Betriebskrankenkassen solche Verträge. Vgl. Das Erste - Plusminus (2005).

${ }^{362} \mathrm{Vgl}$. Hanseatische Krankenkasse (HEK) (2005).
} 
nationalen System der Krankenversicherung zur Versorgung der Versicherten berechtigt sind. Ein finanzielles Risiko kann sich für die Patienten bei der Inanspruchnahme im Rahmen der Waren- und Dienstleistungsfreiheit daraus ergeben, dass die Kosten derjenigen Arznei-, Heil- und Hilfsmittel nicht übernommen werden, die in Deutschland von der Erstattung ausgeschlossen sind. Aus diesem Grund ist es auch für die $\mathrm{Pa}$ tienten von Vorteil, wenn ihre Krankenkasse mit der gewählten Einrichtung im Ausland einen Vertrag geschlossen hat. Dieser erleichtert zudem die Abrechnungen mit den ausländischen Einrichtungen und kann gewisse Qualitätsanforderungen sicherstellen.

Da die Mobilität der Patienten entscheidend von ihrem Gesundheitszustand abhängt, ist davon auszugehen, dass eine Inanspruchnahme stationärer Leistungen im Ausland eher bei Vorsorge- als bei Rehabilitationsmaßnahmen in Frage kommt. Hinzu kommt, dass bei Vorsorgemaßnahmen i.d.R. keine direkte Nachbehandlung bzw. Nachsorge notwendig ist daher die Wohnortnähe der Einrichtung von weit geringerer Bedeutung ist als bei Rehabilitationsleistungen. Lediglich in Grenzregionen kann sich die Situation anders darstellen, wenn eine passende ausländische Kureinrichtung für deutsche Patienten besser erreichbar ist als eine entsprechende Einrichtung in Deutschland.

Auch wenn die Versicherten für die Inanspruchnahme einer Kur im Ausland keine Genehmigung ihrer Krankenkasse entsprechend der Verordnung 1408/71/EWG benötigen, steht es ihnen dennoch frei, diesen Verfahrensweg zu wählen, wenn die gleiche oder eine für den Versicherten ebenso wirksame, dem allgemein anerkannten Stand der medizinischen Erkenntnisse entsprechende Behandlung nicht rechtzeitig bei einer Vertragseinrichtung im Inland durchgeführt werden kann. In diesen Fällen erhalten die Versicherten die Leistungen vom ausländischen Träger als Sachleistungen, müssen aber die im Ausland anfallenden Zuzahlungen tragen, die die deutschen Selbstbehalte deutlich übersteigen können. So haben sie beispielsweise in Frankreich 30 Prozent der Kosten einer Thermalkur zu übernehmen. ${ }^{363}$ Eine zusätzliche Erstattung könnte hier aber aufgrund der Entscheidungen des EuGH in den Rechtssachen „Vanbraekel“ und „Watts" möglich werden. ${ }^{364}$ Finanziell attraktiv kann eine Inanspruchnahme im Ausland auf Basis der Verordnung 1408/71/EWG sein, wenn dort niedrigere Selbstbehalte anfallen oder - im Fall von ambulanten Maßnahmen - die Unterbringungskosten geringer sind als in Deutschland. Die im Rahmen der Maßnahme erstattungsfähigen Leistungen richten sich nach dem ausländischen Leistungskatalog, wodurch die Gefahr, unwissentlich Arznei-, Heil- und Hilfsmitteln in Anspruch zu nehmen, deren Kosten nicht von der Krankenkasse übernommen werden, nicht gegeben ist. Aufgrund der genannten, sehr restriktiven Voraussetzungen für eine Genehmigung bieten die Verordnung 1408/71/EWG jedoch lediglich in Ausnahmefallen eine Möglichkeit für eine Kur im Ausland.

Grundsätzlich besteht bei den GKV-Versicherten ein großes Interesse, Kuren auch grenzüberschreitend nachzufragen. Eine Befragung der Techniker Krankenkasse unter ihren Versicherten ergab, dass sich 67 Prozent der Befragten vorstellen können, Kuren

${ }^{363}$ Vgl. Service Public. Portail de l'Administration Française (2006).

${ }^{364} \mathrm{Vgl}$. zur Darstellung dieser Urteile Abschnitt 2.3.3.1. 
auch in ausländischen Einrichtungen wahrzunehmen. ${ }^{365}$ Die Attraktivität dieser Angebote für die Versicherten und Krankenkassen spiegelt sich auch in den Daten zur Inanspruchnahme wider: Im Jahre 2004 wurden von den Krankenkassen rund ambulante 30.000 Kuren im europäischen Ausland bezahlt. Gemessen an den ca. 118.000 ambulanten Vorsorgemaßnahmen, die in Deutschland genehmigt wurden, beträgt der Anteil der Auslandskuren damit rund 25 Prozent. ${ }^{366}$ Insbesondere hinsichtlich der ambulanten Vorsorgemaßnahmen besteht damit bereits ein intensiver grenzüberschreitender Wettbewerb der Kureinrichtungen, den vor allem Orte in Grenznähe zu den osteuropäischen Mitgliedstaaten zu spüren bekommen und der sich auf die Kosten und die Qualität der in Deutschland erbrachten Leistungen positiv auswirken kann.

Während Kuren zu Lasten der Krankenkassen bereits heute auf Basis der europäischen Grundfreiheiten grundsätzlich auch im Ausland nachgefragt werden können, gilt dies für Rehabilitationsmaßnahmen anderer Finanzierungsträger nicht. Die Regelungen des SGB V sind auf diese Träger nicht anwendbar. Die Kostenübernahme für im Ausland in Anspruch genommene Leistungen richtet sich hier nach § $18 \mathrm{~S}$. 1 SGB IX, wonach eine Erstattung nur dann möglich ist, wenn die Erbringung der Leistung im Ausland wirtschaftlicher ist. Unter Berücksichtigung der „Kohll/Decker"-Rechtsprechung wird diese Regelung von verschiedenen Autoren als europarechtswidrig betrachtet, da das Erfordernis einer wirtschaftlicheren Erbringung ausländische Leistungsanbieter gegenüber Inländern benachteiligt. ${ }^{367}$ Von Bedeutung ist in diesem Zusammenhang auch das Urteil des EuGH in der Rechtssache „Leichtle“ vom 18.03.2004. ${ }^{368}$ Der Gerichtshof hatte darin in Bezug auf das Beihilfesystem, das einen Teil der Krankheitskosten der deutschen Beamten absichert, über eine Regelung zu entscheiden, die die Kostenübernahme bei einer in einem anderen Mitgliedstaat durchgeführten Kur von einer vorherigen Anerkennung der Beihilfefähigkeit abhängig machte. Demnach konnte eine Genehmigung nur dann erteilt werden, wenn die geplante Kur wegen wesentlich größerer Erfolgsaussichten in diesem anderen Mitgliedstaat zwingend notwendig war. Der EuGH kam zu dem Ergebnis, dass eine solche Regelung die Dienstleistungsfreiheit einschränkt und damit gegen europäisches Recht verstößt, da das Erfordernis größerer Erfolgsaussichten ausländische Anbieter diskriminiert. Der Gerichtshof sah hier - anders als bei Krankenhausleistungen - keine konkreten Anhaltspunkte, den Genehmigungsvorbehalt mit der Gefahr für die finanzielle Stabilität des Systems der sozialen Sicherheit zu rechtfertigen. Ebenso sah er ihn nicht als notwendig an, um in Deutschland einen bestimmten Umfang der Versorgung und eine bestimmte Kompetenz aufrecht zu erhalten. ${ }^{369}$ Dementsprechend kann eine Kostenübernahme für einen Kuraufenthalt im Ausland nicht mehr von einer größeren Wirtschaftlichkeit oder Wirksamkeit abhängig gemacht werden. Verschiedene Gründe sprechen jedoch dafür, dass auch bei einer Anpassung der entsprechenden Regelungen im SGB IX an europäisches Recht der Anteil der von den zuständigen Trägern finanzierten Maßnahmen im

\footnotetext{
${ }^{365} \mathrm{Vgl}$. Techniker Krankenkasse (TK) (2003), S. 67.

${ }^{366} \mathrm{Vgl}$. gesundheit.de (2005).

${ }^{367} \mathrm{Vgl}$. Fuchs (2005), S. 266; Kingreen (2006), S. $214 \mathrm{f}$.

${ }^{368} \mathrm{Vgl}$. Europäischer Gerichtshof (2004c).

${ }^{369} \mathrm{Vgl}$. Europåischer Gerichtshof (2004c), Rn. 46 und 47.
} 
Ausland eher gering bleiben wird. Zum einen obliegt die Auswahl der Einrichtung dem jeweiligen Finanzierungsträger, so dass dieser einen großen Einfluss auf den Ort des Leistungsgeschehens behält - im Fall ambulanter Rehabilitationsmaßnahmen ist sogar eine wohnortnahe Erbringung vorgeschrieben. Zum anderen ist davon auszugehen, dass der Gesundheitszustand von Patienten, die eine Maßnahme der medizinischen Rehabilitation benötigen, durchschnittlich schlechter ist als bei Teilnehmern an Vorsorgemaßnahmen. Dieser Umstand wird die Bereitschaft der Patienten, sich für einen stationären Aufenthalt ins Ausland zu begeben, stark einschränken.

Für deutsche Krankenkassen ist die zunehmende Inanspruchnahme von Kuren in Osteuropa zunächst mit der Möglichkeit verbunden, die Ausgaben für Vorsorge- und Rehabilitationsleistungen zu senken. Bei einer Substitution von Inlands- durch Auslandsleistungen ist jedoch eine Situation denkbar, die mit negativen finanziellen Konsequenzen für die deutschen Krankenkassen verbunden wäre. ${ }^{370}$ Eine Verlagerung der Nachfrage in größerem Ausmaß ins Ausland kann zu einer Unterauslastung der inländischen Einrichtungen führen, die deshalb z. T. nicht mehr rentabel arbeiten können. Wenn eine Schließung nicht möglich ist, weil ansonsten eine flächendeckende Versorgung der Versicherten in bestimmten Bereichen gefährdet ist oder politische Gründen dagegen stehen, wird eine Erhöhung der Pflegesätze und/oder der Zuzahlungen der Versicherten notwendig, wenn die höheren durchschnittlichen Kosten pro Patient im Inland nicht durch wesentlich geringere Pro-Kopf-Kosten im Ausland ausgeglichen werden können. Höhere Pflegesätze könnten - zumindest rein theoretisch - eine Beitragssatzsteigerung nach sich ziehen. Letztendlich würde die gewachsene Zahl von Auslandskuren damit $\mathrm{zu}$ einer Mehrbelastung einzelner Patientengruppen (Kur- und Rehabilitationspatienten durch Erhöhung der Zuzahlungen) oder aller Versicherten (durch Beitragssatzsteigerungen) führen. Eine solche Beitragssatzerhöhung könnte nach den Urteilen des EuGH eine Beschränkung der Dienstleistungsfreiheit rechtfertigen, wenn nicht weniger restriktive Instrumente zur Verfügung stehen, um die finanzielle Stabilität des deutschen Gesundheitssystems zu erhalten. ${ }^{371}$

Dieser Effekt darf allerdings nicht überschätzt werden. Im Jahre 2004 betrug der Anteil der Ausgaben für ambulante und stationäre Kuren 0,4 Prozent der gesamten GKVLeistungsausgaben ${ }^{372}$; dies entspricht Ausgaben in Höhe von rund. 0,5 Mrd. $\epsilon^{373}$ Wenn man davon ausgeht, dass der eben beschriebene Zusammenhang zu Ausgaben in doppelter Höhe führen würde, ergäbe sich daraus eine Erhöhung des durchschnittlichen Beitragssatzes um 0,05 Prozentpunkte. ${ }^{374}$

\footnotetext{
${ }^{370} \mathrm{Vgl}$. Wille/Knerr (2000), S. $277 \mathrm{f}$.

${ }^{371}$ Vgl. Domscheit (1998), S. 249

${ }^{372}$ Kuren für Mütter und Väter sowie Anschlussheilbehandlungen bleiben dabei außer Betracht, da deren Erbringung im Ausland mit gravierenden Problemen verbunden ist und darum kein nennenswertes Ausmaß erreichen wird.

${ }^{373}$ Die Leistungsausgaben der GKV betrugen im Jahre 2004 insgesamt 131,2 Mrd. $€$. Vgl. Bundesministerium für Gesundheit (BMG) (2005), Tabelle 10.6.

${ }^{374}$ Ein Beitragssatzpunkt entspricht Einnahmen von rund 9,7 Mrd. $€$. Vgl. Bundesministerium für Gesundheit (BMG) (2006b).
} 
Zudem sind Entwicklungen denkbar, die dem oben beschriebenen Zusammenhang entgegenwirken. Zunächst kann im europäischen Rahmen die Attrahierung von ausländischen Patienten die gesunkene Inlandsnachfrage ausgleichen, wobei es eine Rolle spielt, inwieweit Kuren und Rehabilitation zu den Leistungen ausländischer gesetzlicher Leistungskataloge zählen bzw. inwieweit ausländische Gäste bereit sind, diese Leistungen privat zu bezahlen und welches Ansehen das deutsche Kurwesen im Ausland genießt. Da die deutschen Einrichtungen in einem Preiswettbewerb mit den Kurorten in Osteuropa nicht bestehen könnten, bietet es sich an, die Qualität der Leistungen als Wettbewerbsparameter zu nutzen. Eine zweite Möglichkeit, relevante Kostensteigerungen zu verhindern, besteht in einer frühzeitigen Planung der Kapazitäten. Auch wenn die Schließung ganzer Einrichtungen u.U. unerwünscht ist, kann möglicherweise die Rentabilität einzelner Einrichtungen durch die Reduktion der Bettenzahl erhalten bleiben.

Da der Anteil der durch die öffentlichen Träger finanzierten Aufenthalte rückläufig ist, gewinnen privat organisierte und finanzierte Kuraufenthalte für die deutschen Heilbäder und Kurorte zunehmend an Bedeutung. Im Jahre 2004 besuchten 17 Mio. Gäste deutsche Heil- und Kurorte, wobei nur knapp 2 Mio. Gäste Maßnahmen in Anspruch nahmen, die durch die sozialen Sicherungssysteme finanziert wurden. ${ }^{375}$ Durch diese relativ große Abhängigkeit der deutschen Kureinrichtungen von privat finanzierten Kuren ist für eine Abschätzung der Auswirkungen der Europäischen Integration auch eine Betrachtung dieses Bereichs von Bedeutung. Die Möglichkeit einer privat finanzierten Inanspruchnahme wird von den deutschen und ausländischen Kurorten zunehmend angeboten und beworben und gewann aufgrund des eher restriktiven Genehmigungsverhaltens der Krankenkassen in den letzten Jahren fur die Patienten an Bedeutung.

Genaue Zahlen zu privat finanzierten Aufenthalten von Deutschen in ausländischen Kurorten sind nicht zu ermitteln. Einzelne Beispiele sprechen aber dafür, dass insbesondere osteuropäische Orte in den letzten Jahren an Attraktivität gewonnen haben. So stieg die Zahl deutscher Gäste im tschechischen Karlsbad von 9.624 im Jahre 1996 auf 22.088 im Jahre 2004 an. ${ }^{376}$ Verschiebt sich die Nachfrage zunehmend ins Ausland, ergeben sich zwar keine direkten Auswirkungen für die gesetzlichen Krankenkassen, die an der Planung und Finanzierung nicht beteiligt sind. Es dürfte jedoch für die Entwicklung der deutschen Kureinrichtungen eine bedeutende Rolle spielen, wo der überwiegende Teil dieser Nachfrage wirksam wird. Eine Abwanderung deutscher Gäste könnte übrigens durch die Attrahierung ausländischer Besucher kompensiert werden. Im Jahre 2005 betrug ihr Anteil an allen Gästen in Heil- und Kurorten ca. 8,5 Prozent. Dieser Anteil konnte gegenüber 2004 allerdings nicht gesteigert werden. Die Zahl aller Gäste stieg zwar in beiden Jahren an, aufgrund einer sinkenden durchschnittlichen Aufenthaltsdauer ging jedoch die Zahl der Übernachtungen leicht zurück. ${ }^{377}$ Für die deutschen Kureinrichtungen haben sich durch den Rückgang der durch die Sozialver-

\footnotetext{
${ }^{375}$ Vgl. Deutscher Heilbäderverband (DHV), (2005).

${ }^{376} \mathrm{Vgl}$. Karlovy (Karlsbad), (2006).

${ }^{377} \mathrm{Vgl}$. Deutscher Tourismusverband (2006), S. 16.
} 
sicherungsträger finanzierten Maßnahmen sowie die Konkurrenz ausländischer Kurorte damit stark veränderte wirtschaftliche Rahmenbedingungen ergeben, die auch in Zukunft die deutsche Kurlandschaft beeinflussen werden.

Betrachtet man die Entwicklung insgesamt, so bleibt festzuhalten, dass für die Versicherten Kuren im Ausland insbesondere aufgrund attraktiver äußerer Bedingungen und/oder geringerer Kosten grundsätzlich interessant sind. Gerade bei ambulanten Kuren - unabhängig davon, ob sie privat oder durch die Krankenkassen finanziert werden - können die Preisunterschiede zugunsten osteuropäischer Kurorte dazu beitragen, die Unterbringungs- und Verpflegungskosten deutlich zu reduzieren. Dabei ist es für die Patienten vorteilhaft, wenn ihre Krankenkasse einen Vertrag mit den ausländischen Kureinrichtungen geschlossen hat, auch wenn dies für eine Kostenübernahme nicht zwingend notwendig ist. Die vertragliche Bindung erleichtert aber die Abrechnung der Leistungen und bietet die Möglichkeit, gewisse Qualitätsanforderungen sicherzustellen. Aufgrund der bestehenden Anreize wird erwartet, dass sich die Inanspruchnahme von Kuren in den folgenden Jahren verstärkt ins (ost-)europäische Ausland verlagern wird. Bisher zeichnet sich keine dramatische Verschiebung ab, da auch die Rechtsunsicherheit für die Patienten noch sehr groß ist. ${ }^{378}$ Wenn die Bedeutung dieses Problems aufgrund wachsender Erfahrungen und zunehmenden Kooperationen zwischen ausländischen Anbietern und deutschen Krankenkassen reduziert werden kann, ist davon auszugehen, dass die Attraktivität einer Auslandskur für die Patienten deutlich größer sein wird als zum derzeitigen Zeitpunkt. Dies muss aber nicht nur negative Auswirkungen auf das deutsche Kurwesen haben. Durch die vergleichsweise hohe Qualität der deutschen Einrichtungen ist eine Kur in Deutschland für andere EU-Bürger attraktiv, so dass auf diese Weise eine Abnahme der inländischen Nachfrage kompensiert werden könnte. Grenzen bei der Inanspruchnahme von Rehabilitationsmaßnahmen, die von anderen Leistungsträgern als den gesetzlichen Krankenkassen finanziert werden, setzen die derzeit geltenden rechtlichen Regelungen. Die Regelungen des SGB IX sind jedoch aufgrund der Forderung des Nachweises einer größeren Wirtschaftlichkeit von Auslandsleistungen als Voraussetzung für die Kostenerstattung höchstwahrscheinlich europarechtswidrig.

${ }^{378} \mathrm{Vgl}$. Merten (2004b), S. 1942. 


\subsection{Arzneimittel- und Apothekenmarkt}

\subsubsection{Arzneimittelmarkt}

Arzneimittel nehmen in der modernen Gesundheitsversorgung eine wichtige Stellung ein, was u.a. daran zu erkennen ist, dass die Therapie mit Arzneimitteln die am häufigsten angewandte Behandlungsform bei Krankheiten ist. ${ }^{379}$ Aus ökonomischer Sicht weist das Gut Arzneimittel einige Besonderheiten auf, die zu Abweichungen vom gängigen Marktmodell führen.

Die Contergan-Katastrophe Anfang der 60er Jahre zeigte, dass im Arzneimittelbereich Maßnahmen zur Überprüfung der Sicherheit in größerem Umfang notwendig sind als bei den meisten anderen Gütern, weshalb beispielsweise das deutsche Arzneimittelgesetz (AMG) vor der Marktzulassung eines Medikaments einen Nachweis seiner Qualität, Wirksamkeit und Unbedenklichkeit verlangt. Um die Patienten vor möglichen $\mathrm{Ge}-$ fahren der Arzneimittel zu schützen, darf darüber hinaus ein großer Teil der Medikamente nur aufgrund einer ärztlichen Verordnung an die Patienten abgegeben werden, da der Arzt über das notwendige Fachwissen bezüglich eines sinnvollen Einsatzes verfugt und Nutzen und Gefahren besser abschätzen kann als der Patient selbst. Diese Maßnahme führt aber auch dazu, dass für rezeptpflichtige Arzneimittel die Nachfrageentscheidung nicht von den Konsumenten selbst getroffen wird. Durch den großen Anteil krankenversicherter Menschen an der Bevölkerung ist zudem der Konsum der Medikamente i.d.R. von deren Finanzierung weitgehend getrennt, so dass die Nachfrage nach ärztlich verordneten Arzneimitteln nahezu preisunelastisch reagiert. Da auf diese Weise keine ausreichende Abwägung zwischen Kosten und Nutzen stattfindet, ergibt sich aus allokativer Sicht ein ineffizient hoher Arzneimittelverbrauch, der in allen Ländern der EU begrenzende staatliche Maßnahmen nach sich zieht. Lediglich im Selbstmedikationsmarkt entscheidet der Verbraucher selbst über Nachfrage, Konsum und Finanzierung des Arzneimittels, so dass in diesem Bereich aus theoretischer Sicht - abgesehen von Maßnahmen zur Arzneimittelsicherheit - keine regulativen Eingriffe notwendig sind.

Ein weiteres Charakteristikum des Arzneimittelmarktes stellen die sehr hohen Aufwendungen dar, die die Arzneimittelhersteller für die Entwicklung neuer Arzneimittel tragen müssen. Um ihnen den Anreiz zu Forschung und Entwicklung zu erhalten, existieren in den Industrienationen Patentschutzregelungen, die ein zeitlich begrenztes Monopol gewähren, das einen Preiswettbewerb weitgehend verhindern und einen Preisaufschlag auf die Grenzkosten zur Amortisation der Forschungs- und Entwicklungskosten ermöglichen soll. Erst nach Ablauf dieser Schutzfrist ist ein Wettbewerb mit Nachahmerpräparaten möglich und auch erwünscht.

Aus europarechtlicher Sicht ist für den Arzneimittelmarkt die Warenverkehrsfreiheit von herausragender Bedeutung. Die Union wird tätig, wenn nationales Recht einem grenzüberschreitenden innergemeinschaftlichen Marktzutritt entgegensteht und somit

${ }^{379}$ Vgl. Rosenbrock/Gerlinger (2004), S. 163. 
zur Gewährleistung der vier Grundfreiheiten angeglichen werden muss. ${ }^{380}$ Zahlreiche europäische Richtlinien und Verordnungen - z.B. zur Arzneimittelzulassung oder zum Patentschutz - haben daher das Ziel, einen einheitlichen europaweiten Markt für Arzneimittel zu schaffen, um die Konkurrenzfähigkeit der europäischen Arzneimittelindustrie zu erhalten und zu verbessern. Daneben spielt die Vorabharmonisierung von Maßnahmen zur Produktsicherheit, die die Patienten und die Gesellschaft vor den gesundheitlichen und wirtschaftlichen Folgen von unerwünschten Arzneimittelwirkungen schützen sollen, im Arzneimittelmarkt eine größere Rolle als in den meisten anderen Sektoren. ${ }^{381} \mathrm{Da}$ aber für den Arzneimittelmarkt das Subsidiaritätsprinzip gilt, verbleibt die Regelungskompetenz hinsichtlich der Verordnungsfähigkeit und der nationalen Kostendämpfungsmaßnahmen bei den Mitgliedstaaten, so dass die EU keine Zuständigkeit für eine vollständige Harmonisierung der einzelstaatlichen arzneimittelspezifischen Regulierungen besitzt. Dies zeigt den Konflikt, in dem sich die europäische Arzneimittelpolitik befindet. Zum einen wird beispielsweise bei der Zulassung und dem Patentschutz ein einheitlicher Markt mit gleichen Zugangsbedingungen angestrebt, der dazu führt, dass Arzneimittel europaweit in vergleichbarer Qualität angeboten werden, zum anderen unterliegen die Preisbildung und Erstattung von Arzneimitteln den Regulierungen der einzelnen Mitgliedstaaten, was - zumindest für den großen Teilbereich der im Rahmen der Sozialversicherungssysteme erstattungsfähigen Medikamente - zu hohen Preisdifferenzen zwischen den europäischen Ländern fuhrt.

In den folgenden Abschnitten werden zunächst die europarechtlichen Maßnahmen dargestellt, die durch eine Harmonisierung nationaler Regelungen zur Durchsetzung der Grundfreiheiten und damit zu einem einheitlichen europäischen Arzneimittelmarkt beitragen sollen. Die daran anschließende Übersicht über mögliche Arzneimittelpreisregulierungen, ihre Anwendung in der EU und die daraus resultierenden Preisdifferenzen verdeutlicht den oben beschriebenen Zielkonflikt zwischen freiem Binnenmarkt und nationaler Regelungskompetenzen, der zu verschiedenen - weitgehend jedoch erfolglosen - Versuchen auf europäischer Ebene geführt hat, eine Angleichung der nationalen Maßnahmen zu erreichen. Darum bleibt der aus den Preisdifferenzen resultierende Anreiz zu Re- und Parallelimporten bestehen, die aufgrund der Warenverkehrsfreiheit innerhalb der EU zulässig sind. ${ }^{382}$ Sie dienen den Mitgliedstaaten ebenfalls als Maßnahme zur Kostensenkung, sind aber aufgrund der negativen Auswirkungen auf die innovativen Arzneimittelhersteller differenziert zu betrachten. Zur Förderung eines einheitlichen europäischen Binnenmarktes für Arzneimittel hat die EU in den vergangenen Jahren auch verschiedene Regelungen in Bezug auf Nachahmerpräparate erlassen, die zum Abschluss des Kapitels betrachtet werden.

${ }^{380}$ Vgl. Boroch (1994), S. 185.

${ }^{381}$ Vgl. Boroch (1994), S. 186.

${ }^{382}$ Vgl. dazu Abschnitt 2.3.2. 


\subsubsection{Europäische Marktzulassungsregelungen}

Den ersten Schritt zu einem europäischen Markt für Arzneimittel unternahm die EWG im Jahre 1965 mit der Richtlinie 65/65/EWG zur Angleichung der Rechts- und Verwaltungsvorschriften über Arzneispezialitäten. Aufbauend auf diese Richtlinie wurden zahlreiche Ergänzungen und Modifikationen erlassen, die der Vereinfachung und Koordination der Marktzulassung von Arzneimitteln dienten. ${ }^{383}$ Die Zweite Richtlinie zur Angleichung der Rechts- und Verwaltungsvorschriften über Arzneispezialitäten (75/319/EWG) bildete dann die Grundlage für zwei Verfahren, die die Marktzulassung von Arzneimitteln vereinfachten: ${ }^{384}$ Das Mehrstaatenverfahren ermöglichte eine gegenseitige Anerkennung nationaler Zulassungsanträge, während das Konzertierungsverfahren eine Vereinfachung der Marktzulassung für gen- und hochtechnologische Arzneimittel zum Ziel hatte. Im Rahmen des Maßnahmenkatalogs zur Schaffung des einheitlichen Binnenmarkts und in Reaktion auf Schwierigkeiten mit dem Mehrstaatenverfahren wurden dann zu Beginn der 90er Jahre Regelungen für ein neues Zulassungssystem geschaffen. Demnach stehen für die Arzneimittelzulassung drei mögliche Verfahren zur Verfügung: ${ }^{385}$

Das zentrale Verfahren nach Verordnung 2309/93/EWG ${ }^{386}$ gilt seit 1995 und war zunächst nur für Arzneimittel vorgeschrieben, deren Herstellung mit Hilfe biotechnologischer Prozesse erfolgt, konnte aber für andere innovative Arzneimittel freiwillig gewählt werden. In diesem Verfahren stellt der Hersteller des Arzneimittels den Zulassungsantrag direkt bei der europäischen Zulassungsbehörde „European Agency for Evaluation of Medicinal Products" (EMEA), die für alle Mitgliedstaaten bindend über die Zulassung entscheidet. Die Zulassung ist zunächst fünf Jahre gültig und kann danach auf Grundlage einer von der Agentur vorgenommenen Neubeurteilung des Nutzen-Risiko-Verhältnisses grundsätzlich unbegrenzt verlängert werden. ${ }^{387}$ Seit der Revision der europäischen Arzneimittelgesetzgebung im März 2004 gilt das zentrale Verfahren verpflichtend für Arzneimittel mit neuen Stoffen, wenn sie zur Behandlung von Aids, Krebs, Diabetes und neurodegenerativen oder seltenen Erkrankungen bestimmt sind. Es kann fakultativ auch für Arzneimittel mit einem neuen innovativen Wirkstoff beantragt werden. ${ }^{388}$

\footnotetext{
${ }^{383}$ Die Richtlinie 65/65/EWG wurde erst im Jahre 2001 durch die Richtlinie 2001/83/EG des Europäischen Parlaments und des Rates zur Schaffung eines Gemeinschaftskodexes fur Humanarzneimittel aufgehoben.

${ }^{384}$ Vgl. Boroch (1994), S. $189 f$.

${ }^{385}$ Vgl. Spalcke (2004), S. 2 ff.

${ }^{386}$ Diese Verordnung wurde durch die Verordnung 726/2004/EG vom 31. März 2004 zur Festlegung von Gemeinschaftsverfahren furr die Genehmigung und Überwachung von Human- und Tierarzneimitteln und zur Errichtung einer Europäischen Arzneimittel-Agentur aufgehoben.

${ }^{387}$ Diese zeitlich unbegrenzte Verlängerung ist erst seit dem Jahre 2004 durch die Verordnung 726/2004/EG (Art. 14, Satz 2) möglich geworden. Zuvor war die Verlängerung der Zulassung auf weitere fün Jahre begrenzt.

${ }^{388} \mathrm{Vgl}$. Allgemeine Ortskrankenkasse (AOK) (2005a).
} 
Das dezentrale Verfahren der Richtlinie 93/39/EWG ${ }^{389}$ findet seit 1998 Anwendung und gilt für Arzneimittel, die in mehreren Mitgliedstaaten oder der gesamten EU vertrieben werden sollen. Dabei wird der Zulassungsantrag zunächst bei einer nationalen Zulassungsbehörde gestellt und - nach einer positiven Entscheidung - entsprechend dem Prinzip der gegenseitigen Anerkennung eine Zulassung in beliebig vielen weiteren Mitgliedstaaten erteilt. Dieses Verfahren stellt eine Weiterentwicklung des Mehrstaatenverfahrens mit der wesentlichen Änderung dar, dass die Kommission bei Uneinigkeiten zwischen den Mitgliedstaaten ein Schiedsverfahren unter Leitung der EMEA durchführt, dessen Ergebnis für alle EU-Staaten bindend ist. Die weitaus größte Zahl der Arzneimittelzulassungen in der EU entfällt auf dieses Verfahren.

Daneben ist weiterhin eine rein nationale Zulassung für diejenigen Arzneimittel möglich, die lediglich in einem Mitgliedstaat vertrieben werden sollen. Voraussetzung der Zulassung ist u.a. der Nachweis der Wirksamkeit sowie der angemessenen pharmazeutischen Qualität und Unbedenklichkeit. ${ }^{390}$

Die EU unternahm darüber hinaus seit 1985 in weiteren Bereichen Maßnahmen zur Verwirklichung des freien Waren- und Kapitalverkehrs im Arzneimittelmarkt. Hierzu gehören u.a. die Richtlinien ${ }^{391}$

- zur Einhaltung der Prinzipien der „Guten Herstellungspraxis“ (91/356/EWG)392,

- über den Großhandelsbetrieb (92/25/EWG)393,

- über die Einstufung bei der Abgabe von Arzneimitteln (92/26/EWG),

- über die Etikettierung und die Packungsbeilage (92/27/EWG),

- über die Werbung mit Humanarzneimitteln (92/28/EWG),

die zur Schaffung von gesamteuropäischen Rahmenbedingungen beitrugen, die ihrerseits das Inverkehrbringen und den freien Handel von Arzneimitteln insgesamt vereinfachten. ${ }^{394}$

\subsubsection{Schutzrechte}

Neben den Maßnahmen zur Angleichung der Regelungen, die die Zulassung und den Vertrieb von Arzneimitteln betreffen, ist es ein Anliegen der EU, die Patentschutzregelungen zu vereinheitlichen. Diese sind von wesentlicher Bedeutung für die Innovati-

\footnotetext{
${ }^{389}$ In Verbindung mit den Verordnungen 65/65/EWG und 75/319/EWG.

390 Vgl. § 1 AMG.

${ }^{391} \mathrm{Vgl}$. Boroch (1994), S. 193.

${ }^{392}$ Diese Richtlinie wurde aufgehoben durch Richtlinie 2003/94/EG der Kommission vom 8. Oktober 2003 zur Festlegung der Grundsätze und Leitlinien der Guten Herstellungspraxis fur Humanarzneimittel und furr zur Anwendung beim Menschen bestimmte Prüfpräparate.

${ }^{393}$ Diese und die im Folgenden aufgezăhlten Richtlinien wurden zusammengefasst und z.T. geändert durch Richtlinie 2001/83/EG des Europäischen Parlaments und des Rates vom 6. November 2001 zur Schaffung eines Gemeinschaftskodexes für Humanarzneimittel.

${ }^{394} \mathrm{Vgl}$. Boroch (1994), S. 194.
} 
onsanreize im Arzneimittelbereich, weil sie den Herstellern ermöglichen, innovative Arzneimittel für einen bestimmten Zeitraum exklusiv zu vermarkten ${ }^{395}$ Das Patent gewährt damit gewissermaßen ein Monopol auf Zeit, das die Hersteller nutzen können, um die Kosten der langwierigen Forschungs- und Entwicklungsarbeit zu finanzieren, die einen wesentlichen Teil der Gesamtkosten forschender Arzneimittelunternehmen ausmachen. Diese Kosten sind überwiegend versunkene Kosten (,sunk costs"), die nicht wieder rückgängig gemacht werden können. ${ }^{396} \mathrm{Da}$ sie nicht von der Höhe des Outputs abhängen, ist für die Kostenstruktur forschender Arzneimittelhersteller ein hoher Fixkostenanteil typisch. Bei i.d.R. relativ geringen variablen Kosten führt dies dazu, dass auch bei großen Ausbringungsmengen die Durchschnittskosten oberhalb der Grenzkosten liegen. Ließe man in dieser Situation einen ungehinderten Marktzutritt von Nachahmerpräparaten (sog. Generika) zu, die wesentlich geringere Entwicklungskosten verursachen, käme es zu einem Wettbewerb, der - in einem vollkommenen Markt - den Grenzkostenpreis erzwingen würde. Eine solche Preissetzung führt aber zu Verlusten bei den innovativen Herstellern und verhindert eine Amortisation der Kosten für Forschung und Entwicklung. Dies senkt den Anreiz zur Entwicklung weiterer innovativer Produkte und kann langfristig zum Marktaustritt der forschenden Pharmaunternehmen und zu einer geringeren Zahl von pharmazeutischen Innovationen führen. Aus diesem Grund erhalten die Hersteller ein zeitlich befristetes Monopol in Form eines Patents, das den Wettbewerb zwischen innovativen Produkten und Generika ausschließt und einen Anreiz zur Produktion innovativer Arzneimitteln geben soll.

Auf Basis des 1973 in München unterzeichneten Europäischen Patentübereinkommens (EPÜ) wurde die Europäische Patentorganisation (EPO) gegründet, deren Exekutivorgan das Europäische Patentamt (EPA) ist. ${ }^{397}$ Das EPÜ legt ein einheitliches Verfahren für die Patenterteilung fest, auf dessen Grundlage das EPA ein europäisches Patent erteilt, das jeweils in den ausgewählten Mitgliedstaaten der EPO gütig ist, auf verschiedene Erstreckungsstaaten ${ }^{398}$ erweitert werden kann und eine Laufzeit von 20 Jahren hat. Dieses Europäische Patent stellt ein Bündel der nationalen Patente dar, das nach der Erteilung in diese nationalen Patente zerfällt, so dass in jedem Land grundsätzlich weiterhin die nationalen Bestimmungen gelten. Eventuelle Rechtstreitigkeiten werden darum nach dem jeweiligen nationalen Recht entschieden. ${ }^{399}$ Damit existiert im Bereich der EU bereits eine weitgehende Harmonisierung auf diesem Gebiet: Von den derzeit 25 Mitgliedstaaten der EU ist lediglich Malta nicht dem EPÜ beigetreten. ${ }^{400} \mathrm{Al}-$

\footnotetext{
${ }^{395}$ Das Recht der exklusiven Vermarktung bedeutet in diesem Zusammenhang, dass ein Schutz gegen die Markteinfuhrung von Nachahmerprodukten besteht. Der Parallelimport von Arzneimitteln von einem Mitgliedstaat in einen anderen ist jedoch auf Grundlage der Warenverkehrsfreiheit erlaubt (vgl. dazu auch Abschnitt 3.3.1.4.5).

${ }^{396} \mathrm{Vgl}$. im Folgenden Danzon (1998), S. 296.

397 Vgl. im Folgenden Wikepedia (2006).

398 Die Erstreckungsstaaten sind derzeit Albanien, Bosnien-Herzegowina, Kroatien, die ehemalige jugoslawische Republik Mazedonien sowie Serbien und Montenegro.

${ }_{99}$ In Deutschland ist das Patentrecht im Patentgesetz (PatG) kodifiziert.

${ }^{400}$ Allerdings ist Malta als Mitglied der World Trade Organization (WTO) verpflichtet, das Agreement on Trade-Related Aspects of Intellectual Property Rights (TRIPS) einzuhalten, das gewisse Anforderungen an das nationale Patentrecht stellt (z.B. Mindestdauer des Patentschutzes: 20 Jahre).
} 
lerdings stellt das EPÜ ein Instrument zwischenstaatlichen Rechts dar und ist nicht Bestandteil der gemeinschaftlichen Rechtsordnung, obwohl es sein Entstehen der Idee aus den 1960er Jahren verdankt, ein für die gesamte im Entstehen begriffene europäische Gemeinschaft gültiges Patentschutzsystem zu schaffen. ${ }^{401}$ Dieses Vorhaben war jedoch in einem rein gemeinschaftlichen Rahmen nicht zu verwirklichen, so dass die Initiative lediglich in der Unterzeichnung des Übereinkommens mündete, dem nach und nach fast alle Mitgliedstaaten beitraten. Ein zweiter Versuch, ein Gemeinschaftspatent zu schaffen, führte zur Unterzeichnung des „Luxemburger Übereinkommens über das Gemeinschaftspatent" im Jahre 1977, das jedoch nie in Kraft trat, weil es nicht von alle Mitgliedstaaten ratifiziert wurde. Seit 1997 diskutiert nun die Europäische Kommission erneut die Möglichkeit eines Gemeinschaftspatents ${ }^{402}$ und kündigte im Jahre 1999 in einer Mitteilung ${ }^{403}$ eine neuerliche Initiative an, die sie dort in groben Zügen skizzierte. Im Jahre 2000 betonten die Staats- und Regierungschefs auf der Tagung des Europäischen Rates in Lissabon, wie wichtig eine unverzügliche Einfuhrung des Gemeinschaftspatents zur Unterstützung der dort beschlossenen Strategie zur Förderung der Wettbewerbsfähigkeit der EU sei. Doch auch nach mehreren Beratungen steht eine abschließende Entscheidung des Rates über einen entsprechenden Vorschlag der Europäischen Kommission ${ }^{404}$ noch aus und ist in näherer Zukunft aufgrund hartnäckig bestehender Uneinigkeiten in Fragen der Übersetzung der Patentansprüche nicht zu erwarten, ${ }^{405}$ obwohl die Forderung nach einer schnellen Einführung in jüngster Zeit in den Aktionsplan der Kommission zur Wiederbelebung der Lissabon-Ziele Eingang gefunden hat ${ }^{406}$.

Da ein Patent bereits vor der Prüfungs- und Versuchsphase des Arzneimittels bestehen muss, um eine Imitation durch Konkurrenten zu verhindern, ist die tatsächliche Nutzungsdauer geringer als die Laufzeit des Patents. In Deutschland betrug die effektive Nutzungsdauer bei einer Patentlaufzeit von 20 Jahren nach Angaben des Bundesverbandes der pharmazeutischen Industrie (BPI) im Jahre 2004 in vielen Fällen weniger als zehn Jahre ${ }^{407} \mathrm{Um}$ diese Dauer zu verlängern und damit die Abwanderung forschender Arzneimittelhersteller zu verhindern, schuf der Europäische Rat mit der Verordnung 1768/92/EWG vom 18. Juni 1992 ein ergänzendes Schutzzertifikat für Arzneimittel, das den gleichen Schutz bietet wie das Grundpatent. Das Schutzzertifikat verlängert die Nutzungsdauer des Patents um fünf Jahre, wobei die Dauer der Ausschließlichkeit nicht mehr als 15 Jahre nach der ersten Zulassung des Arzneimittels in einem Mitgliedstaat betragen darf. ${ }^{408}$ Bei fristgerechter Anmeldung gilt das Schutzzertifikat ab dem Zeitpunkt, in dem das Grundpatent ausläuft. Die Wahl der ersten Marktzulas-

\footnotetext{
${ }^{401} \mathrm{Vgl}$. Europäische Kommission (2000c), S. 3.

${ }^{402} \mathrm{Vgl}$. Europäische Kommission (1997).

${ }^{403} \mathrm{Vgl}$. Europäische Kommission (1999).

${ }^{404} \mathrm{Vgl}$. Europarische Kommission (2000c).

${ }^{405}$ Derzeit sieht der Kommissionsvorschlag vor, dass die Patentanspruche - der kürzeste, wenngleich wichtigste Teil der Patentanmeldung, da darin die Grenzen des Schutzes festgelegt werden - in alle Amtssprachen der EU übersetzt werden müssen. Vgl. Europäische Union (2004).

${ }^{406} \mathrm{Vgl}$. Europäische Kommission (2005a), S. 8.

${ }^{407} \mathrm{Vgl}$. Bundesverband der pharmazeutischen Industrie (BPI) (2004), S. 15.

${ }^{408}$ Vgl. Kröck (1998), S. $244 f$.
} 
sung in der EU als Beginn der maximalen effektiven Schutzdauer wurde getroffen, um zu verhindern, dass ein Arzneimittel in einem Mitgliedstaat noch Patentschutz genießt, während es in einem anderen bereits patentfrei und damit kopierbar ist. ${ }^{409}$

Durch den EU-Beitritt der osteuropäischen Staaten Estland, Lettland, Litauen, Polen, Slowenien, Slowakei, Tschechien und Ungarn sowie deren Beitritt zum Europäischen Patentübereinkommen gelten die Regelungen zum Europäischen Patent und zum ergänzenden Schutzzertifikat seit einigen Jahren auch in diesen Staaten. Zuvor war die Patentierbarkeit von Arzneimitteln dort nicht vorgesehen.

\subsubsection{Einflussnahmeversuche der EU auf nationale Kostendämp- fungsmaßnahmen}

Wie bereits erwähnt, dienen die bisher darstellten Maßnahmen der Durchsetzung des freien Warenverkehrs in der EU dem Ziel, den grenzüberschreitenden Handel mit Arzneimitteln zu unterstützen. In der Logik des Binnenmarktkonzepts soll dieser Handel einen zusätzlichen wettbewerblichen Angleichungsdruck auf die einzelstaatlichen arzneimittelrechtlichen Regulierungen der Mitgliedstaaten ausüben und auf diese Weise zu einer Harmonisierung der entsprechenden Regelungen beitragen, die zur Realisierung eines einheitlichen europäischen Arzneimittelmarktes notwendig sind. ${ }^{410}$ Trotz der Fortschritte hinsichtlich einer Vereinheitlichung der Zulassungs- und Vertriebsregelungen kann bisher nicht von einem einheitlichen europäischen Binnenmarkt für Arzneimittel gesprochen werden, obwohl die Europäische Union in der Vergangenheit verschiedene Versuche unternommen hat, eine Angleichung der Kostendämpfungsmaßnahmen zu erreichen. Dabei gilt die im Jahre 1990 verabschiedete sog. „Transparenzrichtlinie ${ }^{\star 4 / 11}$ als der weitreichendste Schritt der EU in diese Richtung. Ziel der Richtlinie ist es, europarechtliche Anforderungen an nationalstaatliche Eingriffe in den Arzneimittelmarkt festzulegen, anhand derer die Betroffenen (z.B. die pharmazeutische Industrie) überprüfen können, ob diese Maßnahmen direkte oder indirekte mengenmäßige Beschränkungen darstellen, die die Warenverkehrsfreiheit verletzen würden. ${ }^{412} \mathrm{Da}$ der Bereich der nationalen Kostendämpfungsmaßnahmen und damit auch die Regelungen bezüglich Preissetzung und Erstattung außerhalb des direkten Einflussbereiches der EU liegen, fordert die besagte Richtlinie lediglich, nationale Entscheidungen über Preisbildung und Erstattung durch objektive und nachvollziehbare Kriterien transparent zu machen. De facto legitimiert sie damit nationalstaatliche Eingriffe in die Arzneimittelpreissetzung sowie in die Sozialversicherungssysteme, solange damit keine Diskriminierung von Erzeugnissen aus anderen EU-Mitgliedstaaten verbunden ist. ${ }^{413}$

\footnotetext{
${ }^{409}$ Vgl. Mehnert (1998), S. 187.

${ }^{410} \mathrm{Vgl}$. Boroch (1994), S. 194.

${ }^{41}$ Richtlinie 89/105/EWG des Rates vom 21. Dezember 1988 betreffend die Transparenz von Maßnahmen zur Regelung der Preisfestsetzung bei Arzneimitteln fur den menschlichen Gebrauch und ihre Einbeziehung in die staatlichen Krankenversicherungssysteme.

${ }^{412}$ Vgl. Kröck (1998), S. 242.

${ }^{413}$ Vgl. Boroch (1994), S. $195 f$.
} 
Nach Art. 9 der Richtlinie sollte die Europäische Kommission zwei Jahre nach deren Inkrafttreten über die gewonnenen Erfahrungen berichten und weitergehende Vorschläge erarbeiten. ${ }^{414}$ Den Versuch einer solchen Fortschreibung unternahm die Kommission im Jahre 1991. ${ }^{415}$ Die zentralen Leitlinien dieses Änderungsvorschlags waren Deregulierung und eine Verstärkung des Wettbewerbs. Die Kommission sprach sich darin gegen direkte Preiskontrollen aus und forderte eine größere Produkttransparenz, den Einsatz von Generika sowie die Einführung von Arzneimittelzuzahlungen. Mit Blick auf die Aufteilung der Kompetenz sollte die eigentliche Richtlinie lediglich insoweit geändert werden, dass eine größere Transparenz in den Verfahren zur Preissetzung, Erstattung und Zulassung erreicht würde. In einem Entwurf zu einer ergänzenden Empfehlung unterbreitete die Kommission jedoch explizite Vorschläge an die Mitgliedstaaten, wie eine wettbewerbliche Ausrichtung der Arzneimittelversorgung zu erreichen sei. Gegen diese Empfehlung gab es heftige Widerstände von Seiten der Pharmaindustrie und der Mitgliedstaaten, die auf den Grundsatz der Subsidiarität verwiesen, so dass diese Fortschreibung letztlich nicht realisiert wurde.

Danach gab es weitere Initiativen der EU-Kommission, die u.a. dazu beitragen sollten, eine Angleichung der Preissetzungs- und Erstattungsstrategien und auch der Preise selbst zu erreichen. ${ }^{416}$ Diese Initiativen basierten auf Verhandlungen zwischen der pharmazeutischen Industrie und den Mitgliedstaaten auf europäischer Ebene. ${ }^{417}$ Der sog. Runde Tisch - vom EU-Kommissar Martin Bangemann im Jahre 1996 initiiert als Forum, das neben den offiziellen Institutionen agierte - hatte das Ziel, zu einem Konsens zwischen den Mitgliedstaaten, der Pharmaindustrie und der Europäischen Kommission über die Maßnahmen zu gelangen, die zur Schaffung eines gemeinsamen Marktes im Bereich der Arzneimittel ergriffen werden sollten. Dabei hatten die Vertreter der Industrie die Erwartung, auf diesem Wege höhere Preise für innovative Arzneimittel sowie eine Abmilderung der Effekte des Parallelhandels zu erreichen. Letztlich scheiterten diese Verhandlungen im Jahre 1998 daran, dass die Mitgliedstaaten nicht bereit waren, auf eine Kostendämpfungspolitik mittels direkter Preiskontrollen zu verzichten.

Zwei Jahre nach dem Scheitern des Runden Tisches nahm eine hochrangige Arbeitsgruppe „Innovation und Bereitstellung von Arzneimitteln" - die sog. G10-Arzneimittelgruppe - ihre Arbeit auf. Die Europäische Kommission wollte damit einen Mechanismus schaffen, der unter Berücksichtigung der Kombination aus gemeinschaftlichen und einzelstaatlichen Kompetenzen die Probleme des pharmazeutischen Sektors unter einem neuen Blickwinkel betrachten und kreative Lösungen finden sollte. ${ }^{418} \mathrm{Da}$ Veränderungen in diesem Bereich letztlich nur mit der Zustimmung der Mitgliedstaaten zu erreichen sind, war zu erwarten, dass der G10-Prozess nicht im Vorschlag einer konkreten Gesetzgebung münden würde. Im Jahre 2002 legte die Gruppe ihren Be-

\footnotetext{
${ }^{414}$ Vgl. im Folgenden Kotzian (2003), S. $155 \mathrm{f}$.

${ }^{415} \mathrm{Vgl}$. Europäische Kommission (1991).

${ }^{416} \mathrm{Vgl}$. im Folgenden Kotzian (2003), S. $161 \mathrm{ff}$.

417 Weitere Akteure wie z.B. Konsumenten, Apotheken, Großhändler, Parallelimporteure und Ärzte blieben dabei weitestgehend außen vor.

${ }^{418} \mathrm{Vgl}$. Europäische Kommission (2003b), S. 3.
} 
richt $^{419}$ vor, der sich mit vierzehn Handlungsvorschlägen sowohl an die Kommission als auch an die einzelnen Mitgliedstaaten wandte. Im Hinblick auf die Preissetzungsund Erstattungssysteme der Mitgliedstaaten erarbeitet sie folgende Vorschläge:

- Die Mitgliedstaaten sollen überprüfen, ob die Zeitspanne zwischen Zulassung eines Arzneimittels und der Preissetzungs- bzw. Erstattungsentscheidung den europarechtlichen Anforderungen entspricht. Zudem sollten sie versuchen, in diesem Bereich eine größere Transparenz und Einheitlichkeit zu erreichen.

- Die Mechanismen und Konzepte zur Einstufung von Medikamenten in verschreibungspflichtige und nicht-verschreibungspflichtige sollen mit dem Ziel überprüft werden, die Entwicklung eines wettbewerbsfähigen Marktes für verschreibungsfreie Arzneimittel zu fördern.

- Die Preise von Arzneimitteln, die nicht durch die Sozialversicherungen erstattet werden, sollen dem freien Wettbewerb überlassen werden.

Mit einer Mitteilung ${ }^{420}$ reagierte die Kommission auf die Empfehlungen der G10Arzneimittelgruppe und unterbreitete Vorschläge zu deren Umsetzung. Sie ging dabei im Abschnitt über die Förderung der Marktintegration und über neue Wege zur Ausgabenbegrenzung in den von ihr vorgeschlagenen Aktionen über die Forderungen der Arzneimittelgruppe hinaus. ${ }^{421}$ So kündigte sie die Einleitung eines Reflexionsprozesses an, der mögliche Maßnahmen der Mitgliedstaaten zur Kontrolle ihrer Arzneimittelausgaben untersuchen soll, und unterbreitete bereits sehr konkrete Änderungsvorschläge. Die Kommission präferiert ein System, in dem die Hersteller die Preise für neue Produkte festlegen dürfen, während die Mitgliedstaaten mit der pharmazeutischen Industrie Maßnahmen zur Eindämmung der Pharmaausgaben aushandeln, beispielsweise in Form von jährlichen Rückzahlungen oder Rabatten, die auf Grundlage der mit diesen Produkten auf den nationalen Märkten erzielten Gewinnen berechnet werden.

Angesichts der geltenden Kompetenzverteilung kann ein solcher Vorschlag lediglich als eine mögliche unverbindliche Diskussionsgrundlage gesehen werden und stellt in keiner Form eine bindende Handlungsempfehlung dar. Es fällt jedoch auf, dass die Europäische Kommission seit der Erarbeitung der Transparenzrichtlinie immer wieder versucht, Einfluss auf die Kostendämpfungsmaßnahmen der Mitgliedstaaten im Arzneimittelsektor zu gewinnen. Da dies auf direktem Weg nicht möglich ist, versucht sie ihre Ziele durch von ihr initiierte Verhandlungen und Foren zum Erfahrungsaustausch zu erreichen. In dieser Vorgehensweise zeigen sich Parallelen zur Grundidee der Offenen Methode der Koordinierung ${ }^{422}$, die seit dem Jahre 2000 auf dem Gebiet der sozialen Sicherung angewandt wird, um durch Informations- und Erfahrungsaustausch sowie Benchmarking zwischen den Mitgliedstaaten eine stärkere und schnellere Konvergenz der nationalen Systeme zu unterstützen.

\footnotetext{
${ }^{419}$ High Level Group on Innovation and Provision of Medicines (2002).

${ }^{420}$ Europäische Kommission (2003b).

${ }^{421} \mathrm{Vgl}$. im Folgenden Europäische Kommission (2003b), S. $17 \mathrm{f}$.

${ }^{422} \mathrm{Vgl}$. zur Darstellung dieser Methode Abschnitt 2.5.
} 
Die Empfehlungen im Arzneimittelbereich spiegeln dabei auch die industriefreundliche Einstellung der Kommission wider, deren Vorschläge für die Umgestaltung der Regulierungssysteme regelmäßig eine Freigabe der Arzneimittelpreise beinhalten ${ }^{423}$, um die Wettbewerbsfähigkeit der europäischen Pharmaindustrie zu fördern. Inwieweit das Vorgehen der Kommission erfolgreich sein kann, hängt letztlich weiterhin von der Bereitschaft der Mitgliedstaaten ab, in diesem Prozess zusammenzuarbeiten und eigene Politiken zu überdenken. Angesichts der unterschiedlichen Regulierungsansätze der Mitgliedstaaten, die zum Teil sehr restriktive Maßnahmen vorsehen, und der divergierenden Interessenlage scheint es jedoch unwahrscheinlich, dass die Strategie der EU zu einer schnellen Konvergenz der Systeme hin zu einer wettbewerblichen Ausrichtung führen wird.

\subsubsection{Nationale Kostendämpfungsmaßnahmen im Arzneimittelmarkt}

In keinem Land der Europäischen Union erfolgt die Preisbildung für Arzneimittel völlig ohne staatliche Eingriffe. Die angewandten Systeme sind unterschiedlich ausgestaltet und unterscheiden sich hinsichtlich des Ausmaßes der Kontrolle. Da Arzneimittelpreise durch die Preisbildung der pharmazeutischen Industrie sowie die Regelungen auf Großhandels- und Apothekenebene entstehen, kann eine Beeinflussung der Preise auf nur einer oder auch auf mehreren dieser Stufen erfolgen. Im folgenden Abschnitt wird auf mögliche Regulierungsformen auf der Herstellerebene eingegangen. ${ }^{424}$

Tabelle 4 zeigt überblickartig die möglichen Maßnahmen zur Steuerung der Arzneimittelausgaben. Neben der Beeinflussung der Arzneimittelpreise kann der Staat auch die Menge der zu Lasten der gesetzlichen Krankenversicherung verordneten Arzneimittel begrenzen, indem er beispielsweise das Verordnungsverhalten der Ärzte beeinflusst oder durch Zuzahlungen die Preiselastizität der Patienten erhöht. In der Tabelle werden darum die Maßnahmen zunächst danach unterschieden, ob sie primär auf den Preis oder die verordnete Menge wirken. Bezüglich der Art der Steuerungswirkung wird dann unterschieden, ob ein direkter oder ein indirekter Einfluss auf Preis und/oder Menge besteht.

${ }^{423}$ Eine Kostendämpfung im Bereich der Krankenversicherungssysteme sollte dann über Regelungen bezüglich der Erstattungsfähigkeit und -höhe angestrebt werden.

${ }^{424}$ Vgl. dazu Breyer/Zweifel/Kifmann (2005), S. 474ff. 
Tabelle 4: Instrumente der Kostendämpfung im Arzneimittelsektor

\begin{tabular}{|c|c|c|c|c|}
\hline \multirow[t]{2}{*}{ Steuerungsmaßnahme } & \multicolumn{2}{|c|}{$\begin{array}{l}\text { Primäre } \\
\text { Wirkungsebene }\end{array}$} & \multicolumn{2}{|c|}{$\begin{array}{l}\text { Art der Steuerungs- } \\
\text { wirkung }\end{array}$} \\
\hline & Preis & Menge & direkt & indirekt \\
\hline Staatliche Preisfestsetzung & $\mathrm{x}$ & & $\mathrm{x}$ & \\
\hline $\begin{array}{lll}\begin{array}{l}\text { Staatliche } \\
\text {-senkungen }\end{array} & \text { Preisstopps und } \\
\end{array}$ & $\mathbf{x}$ & & $\mathbf{x}$ & \\
\hline Festbeträge & $\mathbf{x}$ & & $x^{1}$ & $x^{1}$ \\
\hline Arzneimittelbudgets & $\mathbf{x}$ & $x$ & & $\mathbf{x}$ \\
\hline Positiv- und Negativlisten & & $\mathbf{x}$ & & $x$ \\
\hline Ausschluss aus Positivlisten & & $\mathrm{x}$ & $x^{1}$ & $x^{1}$ \\
\hline $\begin{array}{l}\text { Rezeptfreistellung von Arznei- } \\
\text { mitteln }\end{array}$ & & $x$ & & $\mathbf{x}$ \\
\hline Zuzahlungen & & $\mathrm{x}$ & & $\mathrm{x}$ \\
\hline Förderung von Parallelimporten & $\mathbf{x}$ & & & $x$ \\
\hline Förderung von Generika & $\mathrm{x}$ & & & $\mathbf{x}$ \\
\hline
\end{tabular}

' Die Steuerungswirkung von Festbeträgen wird sowohl als „direkt" als auch als ,indirekt" charakterisiert. Direkt wirken sie insofern, als sie die Erstattungsgrenzen für bestimmte Arzneimittel durch die Finanzierungsträger definieren. Indirekt wirken sie, da die Preisbildung nach wie vor den Firmen uberlassen bleibt, denn diese können die Erstattungsgrenze sowohl über- als auch unterschreiten. Analog kann beim Ausschluss eines Arzneimittels aus der Positivliste argumentiert werden, die de facto eine Festsetzung der Erstattungsobergrenze bei $0 €$ darstellt.

Quelle: nach Rosenbrock/Gerlinger (2004), S. 173; eigene Darstellung

\subsection{Direkte Preisregulierung}

Die restriktivste staatliche Beeinflussung der Arzneimittelpreise stellt die Festsetzung von Arzneimittel(höchst)preisen dar, bei der staatliche Stellen die Preise und deren spätere Änderungen genehmigen müssen. ${ }^{1}$ In Europa ist ein solches Vorgehen beispielsweise in Frankreich, Belgien, Griechenland, Luxemburg, Norwegen, Portugal, Spanien und in den meisten Beitrittsstaaten vorgeschrieben. Eine stärkere Einflussnahmemöglichkeit erhalten die Hersteller, wenn ihnen Preisverhandlungen mit der Regierung möglich sind; doch auch in diesen Systemen bleibt die letztendliche Entscheidung über die Höhe der Preise in der Hand der staatlichen Regulierungsbehörden. In vielen Ländern, die eine direkte Preisregulierung vor-nehmen, dienen bei der Bestimmung des inländischen Preises die Preise des Produkts oder ähnlicher Produkte in

' Vgl. im Folgenden Wasem/Greß/Niebuhr (2005), S. 50ff. 
anderen europäischen Staaten als Referenz. In Griechenland wird dabei der jeweils niedrigste Preis in allen EU-Staaten als inländischer Preis festgesetzt. Die meisten anderen Länder wählen dagegen einen Durchschnittspreis aus ausländischen Preisen für den Vergleich. Vielfach erfolgt in den betreffenden Ländern zusätzlich eine Regulierung der Margen auf Großhandels- und Apothekenebene, so dass die Apothekenpreise letztlich staatlich bestimmt sind.

Zum Bereich der direkten Preisregulierungen zählen auch gesetzlich vorgeschriebene Preisstopps oder -reduktionen. Derartige Maßnahmen wurden in den 1990er Jahren mit Ausnahme von Schweden und Luxemburg in allen damaligen EU-Mitgliedstaaten ergriffen. ${ }^{2}$ Dieses relativ interventionistische Instrument fand also auch in Ländern mit einer eher marktwirtschaftlichen Steuerung des Arzneimittelmarktes Anwendung (z.B. in Deutschland, Dänemark und Großbritannien).

\subsection{Indirekte Preisregulierungen}

Eine indirekte staatliche Beeinflussung der Arzneimittelpreise erfolgt in Europa vor allem durch Festbetragssysteme und Renditeregulierungen. Sie lassen den Herstellern grundsätzlich mehr Freiheit bei der Preissetzung als die direkten Regulierungsmaßnahmen.

Die Festbetragssysteme regulieren dabei die Arzneimittelpreise indirekt über die Festsetzung von Höchstgrenzen für die Erstattung durch die sozialen Sicherungssysteme. Zunächst werden Arzneimittel dabei in Gruppen mit ähnlichen therapeutischen Wirkungen oder gleichen Wirksubstanzen zusammengefasst. Dann wird für jede Gruppe ein einheitlicher Erstattungspreis vorgegeben. Voraussetzung für den Einsatz dieses Instruments ist ein hinreichend großer Anteil von Generika am Arzneimittelmarkt, um ausreichend besetzte Festbetragsgruppen bilden zu können. ${ }^{3}$ Die Hersteller können die Preise ihrer Produkte frei wählen, haben dabei aber zu berücksichtigen, dass die Patienten den Differenzbetrag zwischen dem Endverbraucherpreis und dem Festbetrag selbst tragen müssen. Ziel dieser Maßnahme ist es, dass bei gleicher Qualität ein möglichst günstiges Arzneimittel in der Therapie Einsatz findet. ${ }^{4}$ Die Einführung des Festbetragssystems fuihrte in Deutschland zu einem starken Preiswettbewerb oberhalb des Festbetrags, da die Patienten hier extrem preiselastisch reagierten. Im Bereich der festbetragsgebundenen Arzneimittel senkten (fast) alle Hersteller ihre Preise auf das Festbetragsniveau ab, so dass so dass der Preisindex für diese Produkte von 1989 bis 2003 kontinuierlich sank. ${ }^{5}$ Allerdings fielen im Zeitablauf immer weniger Produkte unter diese Regelungen: Während im Jahre 1997 noch 60 Prozent des Arzneimittelumsatzes mit Festbetragsmedikamenten erzielt wurden, waren es im Jahre 2004 nur noch

\footnotetext{
${ }^{2}$ Vgl. Rosian et al. (2001), S. 401.

${ }^{3}$ Vgl. Rosian et al. (2001), S. 392.

${ }^{4} \mathrm{Vgl}$. Wasem/Greß/Niebuhr (2005), S. 55.

${ }^{5}$ Vgl. Nink/Schröder (2005), Abb. 4.7, S. 205. Im Januar 2004 kam es zu einem Anstieg des Preisindexes, der allerdings allein auf die Änderung der Arzneimittelpreisverordnung durch das GMG zurückzufuhren ist, die zu einer Verteuerung der preiswerten Arzneimittel unterhalb des Festbetrags geführt hat. Vgl. Nink/Schröder (2005), S. 206.
} 
35,8 Prozent. $^{6}$ Aus diesem Grund weitete die damalige Bundesregierung mit dem GMG im Jahre 2004 die Festbetragsregelung auf patentgeschützte Arzneimittel ohne therapeutischen Zusatznutzen aus.

Einen Nachteil des deutschen Systems stellt die sinkende Wettbewerbsintensität unterhalb des Festbetragsniveaus dar. ${ }^{7}$ In diesem Bereich übernimmt die gesetzliche Krankenversicherung die Kosten der Arzneimittel bis auf die für die Patienten anfallenden Zuzahlungen, die jedoch für die meisten Medikamente vom Preis unabhängig sind. ${ }^{8}$ Eine Intensivierung des Wettbewerbs auch unterhalb der Erstattungsgrenzen wird beispielsweise im niederländischen System erreicht. ${ }^{9}$ Um die Generikasubstitution und die Abgabe preisgünstiger Arzneimittel zu fördern, darf der Apotheker einen prozentualen Anteil der Differenz zwischen Abgabepreis und Erstattungsgrenze behalten, wenn der Preis des abgegebenen Medikaments unterhalb dieser Grenze liegt. Auf diese Weise erhält er einen Anreiz, das günstigste Produkt zu wählen.

Insgesamt haben Festbetragsregelungen seit ihrer ersten Einführung im Jahre 1989 in Deutschland europaweit an Bedeutung gewonnen: Anfang der 1990er Jahre folgten Dänemark, die Niederlande und Schweden dem deutschen Beispiel; weitere Länder mit Festbetragssystemen sind Belgien, Italien und Spanien.

Als weitere indirekte Preisbeeinflussung lässt die Renditeregulierung den Herstellern im Vergleich zu den bisher vorgestellten Verfahren die größten Freiheiten in ihrer Preissetzung. ${ }^{10}$ Dieses Verfahren findet in Europa lediglich in Großbritannien in Form des Pharmaceutical Price Regulation Scheme (PPRS) Anwendung. ${ }^{11}$ Die Teilnahme an diesem Programm ist für die Firmen freiwillig, allerdings ist es Voraussetzung für die Erstattungsfähigkeit, dass der Preis eines Medikaments dem PPRS unterliegt. Die Produzenten innovativer Arzneimittel können die Preise für ihre Produkte frei setzen, vorausgesetzt, der Gewinn des Unternehmens übersteigt eine zuvor festgesetzte Grenze nicht. Das britische Gesundheitsministerium und der Pharmaverband ABPI (Association of the British Pharmaceutical Industry) handeln diese periodisch bzw. alle funf Jahr für jedes Unternehmen aus. Wenn ein Unternehmen das festgelegte Limit überschreitet, muss es entweder den Überschuss direkt abführen oder in der nächsten Periode die Preise senken. Bei einer Unterschreitung kann das Unternehmen eine Preiserhöhung durchführen. Das Ziel dieser Regulierung ist die Bereitstellung von sicheren und wirksamen Arzneimitteln zu angemessenen Preisen sowie die Unterstützung der forschenden pharmazeutischen Industrie. Die Regelung berücksichtigt die Notwendigkeit eines Gewinnaufschlages auf die reinen Herstellungskosten, um Innovationsanreize zu schaffen. Allerdings erzeugt die Renditeregulierung Anreize zu „kreativer“ Buchfuhrung und einer Verschwendung von Ressourcen, da der zusătzlich erlaubte

\footnotetext{
${ }^{6} \mathrm{Vgl}$. Nink/Schröder (2005), S. 207.

7 Vgl. Danzon (1997a), S. $19 f$.

${ }^{8} \mathrm{Vgl}$. zur Darstellung der Zuzahlungsregelungen in Deutschland Abschnitt 3.3.2.2.

${ }^{9}$ Vgl. Danzon (1997a), S. 20.

${ }^{10} \mathrm{Vgl}$. im Folgenden Wasem/Greß/Niebuhr (2005), S. 56.

"Die Preise für Generika und rezeptfreie Produkte werden in Großbritannien durch das Maximum Price Scheme staatlich festgesetzt. Vgl. Rychlik/Bundesverband der Pharmazeutischen Industrie (BPI) (2003), S. 21.
} 
Gewinn mit der Höhe des Kapitaleinsatzes steigt und es deshalb für die Hersteller unter bestimmten Bedingungen rational ist, den beobachtbaren Kapiteleinsatz über das effiziente Maß hinaus zu erhöhen. Im Ergebnis führte diese Regulierungsform in Großbritannien zu einer Stabilisierung der Arzneimittelpreise auf hohem Niveau.

Grundsätzlich besteht beim Einsatz von Maßnahmen zur Regulierung von Arzneimittelpreisen ein Konflikt zwischen dem Ziel, den Herstellern die Möglichkeit zur Amortisation ihrer Forschungsaufwendungen zu geben, um ihre Innovationsanreize zu erhalten, und dem Wunsch der Krankenkassen und Patienten nach einer Begrenzung des Wachstums der Arzneimittelausgaben. Die vorgestellten Systeme zur direkten Preisregulierung lassen dabei i.d.R. jedoch unberücksichtigt, dass die Aufwendungen für Forschung und Entwicklung international getragen werden müssen. Eine Preissetzung, die dies außen vor lässt, stellt ein Free-Rider-Verhalten von Seiten der nationalen Regierungen da und senkt die Innovationsanreize für die international tätigen forschenden Pharmafirmen. ${ }^{12}$ Zudem erfolgt die direkte Preisfestsetzung trotz gesetzlich festgelegter Kriterien relativ willkürlich. Auch die Bestimmung mittels internationaler Vergleiche bietet durch die bestehenden Unterschiede in den Dosierungsformen und -stärken einen bedeutenden Interpretationsspielraum. Da davon auszugehen ist, dass die Einwohner der europäischen Mitgliedstaaten bezüglich des Arzneimittelkonsums unterschiedliche Präferenzen aufweisen, fehlt die theoretische Begründung für eine Preissetzung mittels internationaler Vergleiche. Darüber hinaus führen die notwendigen Verhandlungen zu Verzögerungen bei der Markteinfuhrung sowie Unsicherheiten bei den Herstellern und haben darum negative Auswirkungen auf die Innovationsbereitschaft der pharmazeutischen Industrie.

Die indirekten Formen der Preisregulierung räumen den forschenden Herstellern gröBere Freiheiten bei der Preissetzung ein und sind somit grundsätzlich innovationsfreundlicher. Herausragend ist in dieser Hinsicht das britische System, das darum aber auch über relativ hohe Arzneimittelpreise verfügt.

\subsection{Maßnahmen zur Mengensteuerung}

Die wirksame Begrenzung der Arzneimittelausgaben setzt neben der Kontrolle der Preise eine Begrenzung der zu Lasten der öffentlichen Finanzierungsträger verordneten Arzneimittelmengen voraus, da ansonsten preisdämpfende Regulierungen oftmals durch Mengenausweitungen kompensiert werden. Als mengenbegrenzende Maßnahmen stehen den staatlichen Regulierungsbehörden Arzneimittelbudgets, der Ausschluss von Medikamenten aus der Erstattung und die Erhebung bzw. Erhöhung von Zuzahlungen zur Verfügung.

Arzneimittelbudgets wurden in den 90er Jahren in zahlreichen europäischen Ländern entweder auf nationaler oder individueller Ebene bei der Pharmaindustrie oder den Ärzten eingesetzt. ${ }^{13}$ In Deutschland existierte darüber hinaus ein regionales Budget auf

\footnotetext{
${ }^{12}$ Vgl. im Folgenden Danzon (1997a), S. 18.

${ }^{13}$ Vgl. Rosian (2002), Tabelle 2.
} 
der Ebene der Kassenärztlichen Vereinigungen. ${ }^{14}$ Die Wirksamkeit dieses Instruments hängt entscheidend davon ab, ob im Fall von Budgetüberschreitungen effektive Sanktionsmöglichkeiten bestehen. Erfahrungen aus Deutschland und Italien zeigen jedoch, dass Regresse und Rückzahlungen der Ärzte oder der pharmazeutischen Industrie kaum durchgesetzt werden können. In Deutschland wurden die Arzneimittelbudgets im Jahre 2002 daher wieder abgeschafft und durch arztindividuelle Arzneimittelrichtgrößen ersetzt, die die Krankenkassen und die Kassenärztlichen Vereinigungen jährlich miteinander aushandeln.

Als Maßnahmen zur Beeinflussung der Zahl der erstattungsfähigen Arzneimittel dienen Negativ- und Positivlisten sowie die Befreiung von Medikamenten von der Verschreibungspflicht ${ }^{15}$. Die öffentlichen Finanzienungstrăger werden entlastet, indem die betreffenden Arzneimittel in den Selbstmedikationsmarkt überführt werden. Daraus resultiert eine stärkere finanzielle Belastung der Patienten, die diese Medikamente ggf. privat bezahlen müssen. Eine stärkere Beteiligung der Patienten an den Arzneimittelausgaben ergibt sich auch über steigende Zuzahlungen, die zum einen dazu dienen, die Preiselastizität der Nachfrage zu erhöhen, um die Menge der nachgefragten Medikamente einzudämmen, zum anderen aber auch zur finanziellen Entlastung der öffentlichen Haushalte beitragen. Der Finanzierungsanteil der privaten Haushalte an den Arzneimittelausgaben hat in den vergangenen Jahren in vielen europäischen Ländern daher zugenommen. ${ }^{16}$

In ihrer Untersuchung kommt Rosian (2002) zu dem Ergebnis, dass im Zeitraum von 1990 bis 2001 fast alle damaligen EU-Staaten (mit Ausnahme Luxemburgs) zur Dämpfung der Arzneimittelausgaben interventionistisch tätig wurden. Dabei fand in den meisten Ländern ein Mix aus staatlichen Regulierungen und marktwirtschaftlichen Elementen Anwendung. Auch Länder mit einer liberaleren Arzneimittelpolitik (z.B. Frankreich, Dänemark und Belgien) setzten dirigistische Maßnahmen zur direkten Preisregulierung ein. Auf der anderen Seite griffen Länder mit starkem staatlichem Einfluss im Arzneimittelmarkt (z.B. Spanien und Italien) mit der Einführung von Referenzpreissystemen vermehrt auf marktwirtschaftliche Elemente zurück. ${ }^{19}$ Tendenziell ist in der EU im erstattungsfähigen Markt eine Bewegung weg von staatlichen Preisfestsetzungen hin zu Festbetragssystemen bzw. Arzneimittelpreisverhandlungen zu beobachten. ${ }^{18}$ Insgesamt betrachtet erscheint bei der Auswahl von Instrumenten zur Kostensenkung eine Kombination aus Maßnahmen zur Preis- und Mengensteuerung

\footnotetext{
${ }^{14} \mathrm{Vgl}$. Abschnitt 3.2.2.3.

is Die Entscheidung, ob ein Arzneimittel der Verschreibungspflicht unterliegt, erfolgt auf Basis von $\S$ 48 AMG. Dieser sieht zum Schutz der Patienten eine Verschreibungspflicht furr solche Stoffe vor, die ohne ărztliche Überwachung die menschliche Gesundheit auch bei bestimmungsgemäßem Gebrauch gefăhrden können sowie fur alle Arzneimittel, die Stoffe mit nicht allgemein bekannten Wirkungen oder Zubereitungen solcher Stoffe enthalten. (Vgl. Allgemeine Ortskrankenkasse (AOK) (2005g)).Eine Befreiung von Arzneimitteln aus der Verschreibungspflicht allein aus Kostengründen ist darum nicht möglich. Es ist jedoch davon auszugehen, dass in Zeiten knapper Kassen von Seiten der Politik stärker darauf geachtet wird, bei welchen Medikamenten eine solche Befreiung möglich ist.

${ }^{16} \mathrm{Vgl}$. Rosian (2002), S. 27.

${ }^{17} \mathrm{Vgl}$. Rosian, (2002), Tabelle 2.

${ }^{18} \mathrm{Vgl}$. Rosian et al. (2001), S. 392f.
} 
empfehlenswert zu sein, um zu verhindern, dass Preisregulierungen durch Mengenausweitungen kompensiert werden. ${ }^{19}$

\subsection{Exkurs: Arzneimittelpreise im internationalen Vergleich}

Der internationale Vergleich von Arzneimittelpreisen erfolgt über die Berechung von Preisindizes auf Basis eines zuvor spezifizierten Warenkorbes. Die Zusammenstellung dieses Korbes kann das Ergebnis des Vergleichs erheblich beeinflussen, da der Arzneimittelverbrauch von Land zu Land unterschiedliche Strukturen aufweist, sowohl im Hinblick auf Medikamente eines bestimmten Indikationsbereichs als auch in Bezug auf den Anteil der Generika. Zudem ist es von Bedeutung, welche Preissetzungsebene für den Vergleich herangezogen wird. So ist es denkbar, dass ein Land auf der Herstellerebene relativ geringe Preise aufweist, während die Apothekenabgabepreise z.B. aufgrund großzügiger Handesspannen vergleichsweise hoch ausfallen. Aus diesen Gründen sind die Ergebnisse verschiedener Studien kaum miteinander vergleichbar.

Trotz dieser Einschränkungen sollen die in Tabelle 5 und Tabelle 6 aufgeführten Ergebnisse verschiedener Studien dargestellt werden, um einen groben Eindruck von den internationalen Unterschieden in den Arzneimittelpreisen auf Hersteller- und Apothekenebene zu vermitteln. Sowohl die älteren Studien als auch die Untersuchung des Norwegischen Verbands der Arzneimittelhersteller (LMI) bezüglich der Herstellerabgabepreise aus dem Jahr 2004 zeigen, dass innerhalb der EU z.T. gravierende Preisunterschiede bestanden und bestehen. Dabei spielen Differenzen auf der Herstellerebene für die Anreize zum Parallelimport von Arzneimitteln eine Rolle, ${ }^{20}$ während für die Entscheidung der Patienten, Medikamente aus dem europäischen Ausland zu beziehen, die Apothekenabgabepreise entscheidend sind. ${ }^{21}$ Die deutschen Preise liegen in denjenigen Vergleichen, die einen europäischen Mittelwert angeben, jeweils sowohl auf Hersteller- als auch auf Apothekenebene oberhalb dieses Mittelwerts (vgl. Tabelle 5 und Tabelle 6).

\footnotetext{
${ }^{19}$ Vgl. Rosian (2002), S. 30.

${ }^{20} \mathrm{Vgl}$. Absatz 3.3.1.4.5.

${ }^{21}$ Spürbar für den Patienten sind solche Preisunterschiede allerdings nur im Rahmen der Selbstmedikation oder bei prozentualen Arzneimittelzuzahlungen.
} 
Tabelle 5: Internationaler Vergleich der Herstellerabgabepreise

\begin{tabular}{|l|l|l|l|l|l|}
\hline & $\begin{array}{l}\text { BEUG } \\
(1989)\end{array}$ & $\begin{array}{l}\text { IWI } \\
(1991)\end{array}$ & $\begin{array}{l}\text { Farm- } \\
\text { industria } \\
(1991)\end{array}$ & $\begin{array}{l}\text { BASY } \\
\text { S } \\
(1999)^{1}\end{array}$ & $\begin{array}{l}\text { LMI } \\
(2004)\end{array}$ \\
\hline EG & 100 & 100 & 100 & - & - \\
\hline Belgien & 90 & 91 & 86 & 128 & 89 \\
\hline Dänemark & 132 & - & - & 122 & 101 \\
\hline Deutschland & 105 & 128 & 116 & 100 & 100 \\
\hline Finnland & - & - & - & 134 & 101 \\
\hline Frankreich & 68 & 60 & 57 & 109 & 93 \\
\hline Griechenland & 90 & 49 & - & 85 & 82 \\
\hline Großbritannien & 117 & 123 & 95 & 135 & 112 \\
\hline Irland & 124 & - & - & 133 & 88 \\
\hline Italien & 97 & 92 & 90 & 117 & 88 \\
\hline Luxemburg & - & - & - & 123 & - \\
\hline Niederlande & 132 & 155 & 147 & 124 & 108 \\
\hline Österreich & - & - & - & 108 & 95 \\
\hline Portugal & 68 & 74 & - & 109 & 97 \\
\hline Schweden & - & - & - & - & 95 \\
\hline Spanien & 76 & 60 & - & 88 & 86 \\
\hline
\end{tabular}

1) Vergleichsmaßstab: Devisenkurse. BASYS legte beim Vergleich den deutschen Warenkorb zugrunde. Da ein Warenkorb die Nachfrageverhältnisse im betreffenden Land abbildet und stärker nachgefragte Arzneimittel gemeinhin niedrigere Preise aufweisen, erklart sich das im Gegensatz zu anderen Studien vergleichsweise geringe Preisniveau für Deutschland.

BEUG: Bureau Européen des Unions des Consommateurs; BASYS: Beratungsgesellschaft für angewandte Systemforschung; LMI: Legemiddelindustrif (Norwegischer Verband der Arzneimittelhersteller)

Quelle: Boroch (1994), S. 175; Schneider (1999), S. 57; Verband Forschender Arzneimittelhersteller (VFA) (Hrsg.) (2006), S. 18. 
Tabelle 6: Internationaler Vergleich der Apothekenabgabepreise

\begin{tabular}{|l|l|l|l|l|l|l|l|l|}
\hline & $\begin{array}{l}\text { BEUG } \\
(1989)\end{array}$ & $\begin{array}{l}\text { Test- } \\
\text { Achats } \\
(1989)\end{array}$ & $\begin{array}{l}\text { Die- } \\
\text { ner } \\
(199 \\
0)\end{array}$ & $\begin{array}{l}\text { Farm- } \\
\text { in- } \\
\text { dustria } \\
(1990)\end{array}$ & $\begin{array}{l}\text { ABDA } \\
(1991)\end{array}$ & $\begin{array}{l}\text { Deut- } \\
\text { sche } \\
\text { Bank } \\
(1992)\end{array}$ & $\begin{array}{l}\text { ABDA } \\
(1993)\end{array}$ & $\begin{array}{l}\text { BA- } \\
\text { SYS } \\
(1999)\end{array}$ \\
\hline EG & 100 & 100 & 100 & 100 & 100 & 100 & 100 & - \\
\hline Belgien & 85 & 92 & 68 & 103 & 101 & 86 & 116 & 122 \\
\hline Dänemark & 141 & 129 & 129 & - & 143 & 136 & 133 & 107 \\
\hline Deutschland & 146 & 149 & 127 & 146 & 111 & 142 & 105 & 100 \\
\hline Finnland & - & - & - & - & - & - & - & 112 \\
\hline Frankreich & 68 & 70 & 72 & 64 & 64 & 72 & 63 & 98 \\
\hline Griechenland & 71 & 55 & 74 & - & 86 & 65 & 85 & 69 \\
\hline $\begin{array}{l}\text { Groß- } \\
\text { britannien }\end{array}$ & 110 & 120 & 117 & 123 & 125 & 110 & 123 & 110 \\
\hline Irland & 128 & - & 132 & - & 130 & 120 & 133 & 122 \\
\hline Italien & 78 & 72 & 80 & 99 & 96 & 69 & 96 & 104 \\
\hline Luxemburg & 95 & - & 98 & - & 95 & 72 & 97 & 115 \\
\hline Niederlande & 131 & 165 & 133 & 175 & 134 & 125 & 148 & 106 \\
\hline Österreich & - & - & - & - & - & - & - & 122 \\
\hline Portugal & 61 & 64 & 68 & 0 & 58 & 56 & 67 & 85 \\
\hline Spanien & 69 & 58 & 73 & 68 & 84 & 65 & 94 & 77 \\
\hline
\end{tabular}

") Vgl. Anmerkung 1 zu Tabelle 5.

BEUG: Bureau Européen des Unions des Consommateurs; ABDA: Bundesvereinigung Deutscher Apothekerverbănde; BASYS: Beratungsgesellschaft für angewandte Systemforschung

Quelle: Boroch (1994), S. 173; Schneider (1999), S. 51

Die Arzneimittelpreise sind in jedem europäischen Land mehr oder weniger stark reguliert, so dass diese Differenzen nicht das Ergebnis einer freien Preisbildung darstellen, sondern durch zahlreiche staatliche Eingriffe bestimmt sind. Ein bedeutender Einfluss kommt dabei auch den Umsatzsteuersätzen für Arzneimittel zu, die innerhalb Europas erheblich variieren (vgl. Tabelle 7). Viele europäische Länder erheben auf Arzneimittel einen reduzierten Umsatzsteuersatz. Lediglich Dänemark, Deutschland und die Slowakei besteuern Arzneimittel ausnahmslos mit dem vollen Satz. 
Tabelle 7: Umsatzsteuersätze in ausgewählten europäischen Ländern

\begin{tabular}{|l|l|l|}
\hline Land & Umsatzsteuer für Arzneimittel & $\begin{array}{l}\text { Normalsatz der } \\
\text { Umsatzsteuer }\end{array}$ \\
\hline Belgien & 6,0 & 21,0 \\
\hline Dänemark & 25,0 & 25,0 \\
\hline Deutschland & 19,0 & 19,0 \\
\hline Estland & 5,0 & 18,0 \\
\hline Finnland & 8,0 & 22,0 \\
\hline Frankreich & 2,1 für erstattungsfähige AM & 20,6 \\
& 5,5 für nicht erstattungsfähige AM & \\
\hline Griechenland & 9,0 für verschreibungspflichtige AM & 18,0 \\
\hline Großbritannien & 0,0 für AM im Rahmen des NHS & 17,5 \\
& 17,5 für nicht verschreibungspflichtige AM & \\
\hline Irland & 0,0 für AM zur oralen Anwendung & 21,0 \\
& 21,0 für nicht oral anzuwendende AM & \\
\hline Italien & 10,0 & 19,0 \\
\hline Lettland & 5,0 & 18,0 \\
\hline Litauen & 5,0 & 18,0 \\
\hline Luxemburg & 3,0 & 15,0 \\
\hline Malta & 0,0 & 0,0 \\
\hline Niederlande & 6,0 & 19,0 \\
\hline Österreich & 0,0 bei Übernahme durch die Sozialversicherung & 20,0 \\
\hline Polen & 20,0 bei Übernahme durch den Patienten & \\
\hline Portugal & 22,0 & 7,0 \\
\hline Schweden & 5,0 & 21,0 \\
\hline Schweiz & 0,0 für verschreibungspflichtige AM & 25,0 \\
\hline Slowakei & 25,0 für nicht verschreibungspflichtige AM & \\
\hline Slowenien & 2,4 & 7,6 \\
\hline Spanien & 8,5 & 19,0 \\
\hline Tschechien & 4,0 & 20,0 \\
\hline Ungarn & 5,0 & 16,0 \\
\hline Zypern & 5,0 & 19,0 \\
\hline AM:Arn & 20,0 \\
\hline 0,0 & 15,0 \\
\hline & & \\
\hline
\end{tabular}

AM: Arzneimittel

Quelle: Schneider (1999); European Federation of Pharmaceutical Industrie and Associations (EFPIA) (2000); Bundesverband der Pharmazeutischen Industrie (BPI) (2006)

Den Einfluss der unterschiedlichen Besteuerung von Arzneimitteln und der staatlichen Regulierung der Handelsspannen zeigt Tabelle 8 (siehe Seite 121) exemplarisch für ein verschreibungspflichtiges Arzneimittel mit einem europaweit einheitlichen Herstellerabgabepreis von $10 €$, wobei die Berechnung auf Durchschnittswerten für die 
Handelsspannen auf Großhandels- und Apothekenebene basiert. ${ }^{22}$ Das entsprechende Produkt würde in Deutschland im Ergebnis 19,53 $€$ kosten und wäre damit teurer als in jedem anderen betrachteten Land. Am größten wäre die Differenz im Vergleich zum schwedischen Apothekenabgabepreis, der fast $6 €$ unter dem deutschen läge. Dies zeigt, welche Bedeutung die staatlichen Eingriffe in die Herstellerpreise und Handelsspannen sowie die unterschiedlich hohe Besteuerung für die Arzneimittelpreisdifferenzen haben. Aufgrund dieser staatlichen Eingriffe kann es trotz der Freiheit des Warenverkehrs und der damit verbundenen Möglichkeit der Arbitrage nicht zu einer Angleichung der Preise kommen, obwohl Arzneimittel sehr homogene Produkte sind, die leicht transportiert werden können.

\subsection{Parallel- und Reimporte}

Die vorgestellten Maßnahmen zur Kostendämpfung im Arzneimittelmarkt führen in Verbindung mit der vielfach angewandten Regulierung der Großhandels- und Apothekenspannen zu z.T. deutlichen Differenzen in den Arzneimittelpreisen der EUMitgliedstaaten, die durch die Initiativen der Europäischen Kommission bislang nicht beseitigt werden konnten. Durch diese Differenzen ergibt sich ein ökonomischer Anreiz zum Parallelimport von Arzneimitteln, dessen rechtliche Grundlagen in der EU bereits im Abschnitt 2.3.2 dargestellt wurden und deren Förderung auf nationaler Ebene ebenfalls als Kostendämpfungsmaßnahme dient. Da die Anreize zum Parallelimport von Arzneimitteln umso größer sind, je geringer die Kosten der Importeure für Zulassung und Vertrieb und je größer die Preisunterschiede in den verschiedenen Mitgliedstaaten sind, haben der EU-Beitritt osteuropäischer Staaten, die relativ niedrige Arzneimittelpreisniveaus aufweisen, sowie die Harmonisierung der Anforderungen an Anmeldung, Dosierungsempfehlungen und Kennzeichnung die Rahmenbedingungen für Parallelimporteure verbessert. ${ }^{23}$ Tabelle 9 zeigt die Entwicklung der Parallelimporte am verschreibungspflichtigen Markt in ausgewählten europäischen Ländern. Der Vergleich zeigt, dass sich in den betrachteten Mitgliedstaaten die Entwicklung z.T. recht unterschiedlich vollzogen hat. Die Niederlande wiesen bereits 1997 mit 14,0 Prozent einen relativ hohen Anteil von Parallelimporten am Arzneimittelmarkt auf, der in den Jahren bis 2002 kaum variierte. Auch in Dänemark blieb der Anteil der Parallelimporte relativ konstant. Deutliche Zunahmen waren in Schweden und in Großbritannien zu beobachten. In Deutschland war der Anteil am Apothekenmarkt von 1,7 Prozent im Jahre 1997 auf 7,0 Prozent im Jahre 2002 gestiegen. Eine wichtige Determinante für diesen Anstieg stellt die staatliche Unterstützung von Arzneimittelparallelimporten dar. ${ }^{24} \S 129$ Abs. 1 SGB V verlangt von Apotheken die Abgabe von preisgünstigen importierten Arzneimitteln. Der Rahmenvertrag über die Arzneimittelver-

\footnotetext{
${ }^{22}$ Weitere Mitgliedstaaten der EU konnten bei der Berechnung aufgrund fehlender Daten nicht berücksichtigt werden.

${ }^{23}$ Vgl. Danzon (1998), S. 294.

24 Vgl. z.B. Laschet (2003).
} 
Tabelle 8: Preisdifferenz bei erstattungsfähigen Arzneimitteln durch Handelsaufschläge und Umsatzsteuer (USt) am Beispiel eines Medikaments mit einem europa-einheitlichen Herstellerabgabepreis von $10 €$

\begin{tabular}{|c|c|c|c|c|c|c|c|c|c|c|}
\hline & \multirow{2}{*}{$\begin{array}{l}\text { Hersteller- } \\
\text { preis }\end{array}$} & \multicolumn{2}{|c|}{$\begin{array}{l}\text { Durchschnittl. } \\
\text { Großhandels- } \\
\text { spanne }\end{array}$} & \multirow{2}{*}{$\begin{array}{l}\text { Durch- } \\
\text { schnittl. AEP } \\
\text { (netto) }\end{array}$} & \multicolumn{2}{|c|}{$\begin{array}{l}\text { Durchschnittl. } \\
\text { Apothekenspanne }\end{array}$} & \multirow{2}{*}{$\begin{array}{l}\begin{array}{l}\text { AVP } \\
\text { (netto) }\end{array} \\
\text { in } €\end{array}$} & \multicolumn{2}{|c|}{$\begin{array}{l}\text { USt auf erstattungs- } \\
\text { fähige Arzneimittel }\end{array}$} & \multirow{2}{*}{$\begin{array}{l}\text { AVP } \\
\text { (brutto) }\end{array}$} \\
\hline & & $\begin{array}{l}\text { in } \\
\text { Prozent } \\
\text { des HP }\end{array}$ & in $€$ & & $\begin{array}{l}\text { in Prozent } \\
\text { des AEP }\end{array}$ & in $€$ & & $\begin{array}{l}\text { in Prozent des } \\
\text { AVP (netto) }\end{array}$ & in $€$ & \\
\hline Schweden & 10,00 & 3,09 & 0,31 & 10,31 & 25,00 & 2,58 & 12,89 & 0,00 & 0,00 & 12,89 \\
\hline $\begin{array}{l}\text { Groß- } \\
\text { britannien }\end{array}$ & 10,00 & 14,23 & 1,42 & 11,42 & 20,92 & 2,39 & 13,81 & 0,00 & 0,00 & 13,81 \\
\hline Frankreich & 10,00 & 4,09 & 0,41 & 10,41 & 38,12 & 3,97 & 14,38 & 2,10 & 0,30 & 14,68 \\
\hline Portugal & 10,00 & 12,36 & 1,24 & 11,24 & 25,00 & 2,81 & 14,04 & 5,00 & 0,70 & 14,80 \\
\hline Italien & 10,00 & 10,54 & 1,05 & 11,05 & 28,87 & 3,19 & 14,25 & 10,00 & 1,42 & 15,67 \\
\hline Niederlande & 10,00 & 16,96 & 1,70 & 11,70 & 27,23 & 3,18 & 14,88 & 6,00 & 0,89 & 15,77 \\
\hline Finnland & 10,00 & 4,09 & 0,41 & 10,41 & 40,45 & 4,21 & 14,62 & 8,00 & 1,17 & 15,79 \\
\hline Griechenland & 10,00 & 8,65 & 0,87 & 10,87 & 34,95 & 3,80 & 14,66 & 8,00 & 1,17 & 15,84 \\
\hline Spanien & 10,00 & 10,58 & 1,06 & 11,06 & 38,70 & 4,28 & 15,34 & 4,00 & 0,61 & 15,95 \\
\hline Luxemburg & 10,00 & 15,20 & 1,52 & 11,52 & 46,63 & 5,37 & 16,89 & 3,00 & 0,51 & 17,40 \\
\hline Belgien & 10,00 & 15,00 & 1,50 & 11,50 & 44,93 & 5,17 & 16,67 & 6,00 & 1,00 & 17,67 \\
\hline Dänemark & 10,00 & 7,77 & 0,78 & 10,78 & 41,44 & 4,47 & 15,24 & 25,00 & 3,81 & 19,05 \\
\hline Österreich & 10,00 & 14,49 & 1,45 & 11,45 & 40,65 & 4,65 & 16,10 & 20,00 & 3,22 & 19,32 \\
\hline Deutschland & 10,00 & 14,98 & 1,50 & 11,50 & 46,41 & 5,34 & 16,84 & 16,00 & 2,69 & 19,53 \\
\hline
\end{tabular}

AEP: Apothekeneinkaufspreis, AVP: Apothekenverkaufspreis

Quelle: Österreichisches Bundesinstitut für Gesundheitswesen (ÖBIG) (Hrsg.) (2001); Bundesverband der Pharmazeutischen

Industrie (BPI) (2006), eigene Berechungen 
sorgung aus dem Jahre 2004 verpflichtet Apotheken zu einer Mindestabgabequote in Höhe von 5 Prozent. Der Preisabstand zum Bezugsarzneimittel muss dabei für Arzneimittel mit einem Preis unter $100 €$ mindestens 15 Prozent betragen; für Arzneimittel mit einem Preis ab $100 €$ ist ein Preisabstand von mindestens $15 €$ vorgeschrieben. Ziel des Gesetzgebers ist es, durch die Förderung des Parallelimports von Arzneimitteln die Kosten der gesetzlichen Krankenkassen in diesem Bereich zu senken. Die gesetzlichen Krankenkassen haben im Jahre 2001 ca. 60 Mio. $€$ durch Parallelimporte eingespart. $^{25}$

Wie die folgenden Betrachtungen zeigen, ist aus ökonomischer Sicht jedoch ein differenzierterer Blick auf die Auswirkungen des Parallelimports von Arzneimitteln angebracht.

Tabelle 9: Umsatzanteil der Parallelimporte am verschreibungspflichtigen Markt in ausgewählten europäischen Ländern, 1997-2002 ${ }^{1)}$

\begin{tabular}{|l|l|l|l|l|l|l|}
\hline Land & 1997 & 1998 & 1999 & 2000 & 2001 & 2002 \\
\hline Schweden & $1,9 \%$ & $6,2 \%$ & $7,7 \%$ & $8,6 \%$ & $9,3 \%$ & $10,1 \%$ \\
\hline Dänemark & $9,1 \%$ & $10,0 \%$ & $10,0 \%$ & $10,2 \%$ & $9,9 \%$ & $9,7 \%$ \\
\hline Deutschland & $1,7 \%$ & $1,9 \%$ & $2,3 \%$ & $3,2 \%$ & $4,7 \%$ & $7,0 \%$ \\
\hline Niederlande & $14,0 \%$ & $14 \%$ & $14,5 \%$ & $13,5 \%$ & $14,3 \%$ & $14,0 \%$ \\
\hline Großbritannien & - & $9,5 \%$ & $11,9 \%$ & $13,6 \%$ & $17,1 \%$ & $19,8 \%$ \\
\hline
\end{tabular}

1) Daten und Informationen sind für eine Reihe von Ländern, z.B. Frankreich, Italien und Spanien, nicht verfügbar.

Quelle: Zusammengestellt aus Kanavos/Costa-Font (2005), S. 763.

Wie bereits in Abschnitt 3.3.1.2 ausgeführt, gewährt der Patentschutz dem Hersteller ein zeitlich beschränktes Monopol, um die Amortisation der Forschungs- und Entwicklungskosten zu ermöglichen. Durch dieses Monopol besteht für den Hersteller theoretisch ein Anreiz, den Monopolpreis zu setzen, der seinen Gewinn maximiert. Aus wohlfahrtstheoretischer Sicht ergibt sich daraus jedoch die Notwendigkeit einer Preisregulierung. Wird nun der Nachfragerpreis auf Grenzkostenniveau gesetzt und die entstehenden Verluste der Hersteller über steuerfinanzierte Subventionen gedeckt, können die Arzneimittelhersteller die Forschungs- und Entwicklungskosten decken, ohne dass eine Monopolpreissetzung erfolgt. Die positive Wohlfahrtswirkung dieser $\mathrm{Maßnahme} \mathrm{hängt} \mathrm{aber} \mathrm{stark} \mathrm{davon} \mathrm{ab,} \mathrm{dass} \mathrm{die} \mathrm{zur} \mathrm{Finanzierung} \mathrm{der} \mathrm{Subvention} \mathrm{not-}$ wendige Steuer nicht verzerrend wirkt. Wenn eine solche Kopfsteuer - z.B. aus politischen Erwägungen - nicht realisierbar ist und zudem eine Preisdifferenzierung zwischen verschiedenen Nachfragergruppen mit unterschiedlichen Preiselastizitäten der Nachfrage (z.B. zwischen verschiedenen Ländern) möglich ist, stellen aus theoretischer Sicht sog. Ramsey-Preise die beste Lösung dar. ${ }^{26}$ Ziel dieser Preissetzung ist die

${ }^{25} \mathrm{Vgl}$. Verband der Forschenden Arzneimittelhersteller (VFA) (2004).

${ }^{26} \mathrm{Vgl}$. im Folgenden Danzon (1998), S. 297f. 
Maximierung der Gesamtwohlfahrt unter der Nebendingung, dass die Durchschnittskosten des Herstellers gedeckt sind. Dabei hängt die Höhe der Preissteigerung invers von der Preiselastizität der Nachfrage der verschiedenen Gruppen ab, weshalb diese Preissetzungsregel auch „Regel der inversen Elastizität“ genannt wird: Da bei einer gegebenen Preiserhöhung der Wohlfahrtsverlust umso größer ist, je stärker die Nachfrage auf Preisänderungen reagiert, sollte die Gruppe mit einer hohen Nachfrageelastizität nur einen geringen Aufschlag auf die Grenzkosten tragen, während von der Gruppe mit unelastischer Nachfrage höhere Preise verlangt werden können. ${ }^{27} \mathrm{Je}$ gröBer die Preiselastizität der Nachfrage ist, umso geringer muss der Preisaufschlag auf die Grenzkosten gewählt werden.

In der Europäischen Union ist zu beobachten, dass Länder mit geringen Pro-KopfEinkommen typischerweise relativ geringe Arzneimittelpreise aufweisen, während reichere Staaten eher zu den Hoch-Preis-Ländern zählen. Würde man davon ausgehen, dass diese Preisdifferenzen auf den europäischen Arzneimittelmärkten aus einer Preissetzung gemäß der inversen Elastizitätenregel resultieren - was implizierte, dass höhere Preiselastizitäten in den ärmeren Ländern gelten - wäre ein Parallelimport von Arzneimitteln, der $\mathrm{zu}$ einer Angleichung der Preise führt, aus wohlfahrtstheoretischer Sicht negativ zu werten. Nimmt man an, das weiterhin eine Deckung der Durchschnittskosten des Herstellers gewährleistet ist, so führt die Preisangleichung zu einem Wohlfahrtsverlust durch eine sinkende Konsumentenrente: Zwar steigt durch die Preissenkung die Wohlfahrt der Konsumenten in Hochpreisländern, dieser Gewinn wird jedoch vom Wohlfahrtsverlust der Verbraucher aufgrund der Preissteigerung in den Niedrigpreisländern überkompensiert. Es ist ebenfalls möglich, dass die Preisangleichung zu sinkenden Umsätzen für den Hersteller führt, der dann seine Durchschnittskosten nicht mehr decken kann. Auf diese Weise werden Innovationen weniger attraktiv, so dass dann in der dynamischen Sicht die Gesamtwohlfahrt durch eine geringere Zahl von innovativen Arzneimitteln abnimmt.

In der Realität unterscheiden sich die Märkte für Arzneimittel aufgrund verschiedener Eigenschaften von den Monopolen, für die die Theorie der Ramsey-Preise gilt. ${ }^{28} \mathrm{Zwar}$ erhalten die Anbieter aufgrund des Patentschutzes eine gewisse Marktmacht, doch sind trotz dieses Schutzes reine Monopol selten und zeitlich beschränkt, da zu beobachten ist, dass oftmals bereits wenige Monate nach der Markteinführung eines innovativen Arzneimittels in einer neuen therapeutischen Gruppe Medikamente mit ähnlichen Wirkstoffen bzw. Wirkungen auf den Markt kommen. ${ }^{29}$ Die Pharmafirmen sehen sich also einer gewissen Konkurrenz durch enge, aber nicht vollkommene Substitute ihrer Produkte gegenüber. In diesem Fall kann die vorherrschende Marktform als monopo-

\footnotetext{
${ }^{27}$ Vgl. Brümmerhoff (2001), S. 71.

${ }^{28}$ Vgl. Danzon (1997b), S. 303.

${ }^{29} \mathrm{Vgl}$. Danzon (1998), S. 296.
} 
listische Konkurrenz charakterisiert werden. ${ }^{30}$ Zudem hat jede nationale Regulierungsbehörde lediglich die Kompetenz für die Festsetzung von nationalen Preis- und Erstattungssystemen, während eine Preisregulierung mittels Ramsey-Preisen nur supranational möglich wäre. Die Preisunterschiede zwischen den europäischen Arzneimittelmärkten resultieren darum aus einzelstaatlichen Regulierungen, die vorrangig das Ziel der Kostendämpfung im Gesundheitswesen verfolgen, und entsprechen nicht den optimalen Ramsey-Preisen. ${ }^{31}$ Doch auch unter diesen Voraussetzungen sind die Wohlfahrtseffekte von Arzneimittelparallelimporten nicht eindeutig. Durch den Import der niedrigpreisigen Arzneimittel ergeben sich nicht intendierte grenzüberschreitende Effekte einzelstaatlicher Kostendämpfungs-maßnahmen, da mit der Einfuhr dieser Medikamente quasi auch die zugehörigen Preisregulierungen importiert werden, so dass die Produktionseffizienz der forschenden Hersteller in diesen Mitgliedstaaten gestört und Anreize zu Innovationen behindert werden können. ${ }^{32}$

Diese Überlegungen zeigen, dass die Wirkung von Arzneimittelparallelimporten nicht abschließend zu bewerten ist. Die zahlreichen theoretischen Arbeiten über die Auswirkungen von Parallelimporten auf die Gesamtwohlfahrt ${ }^{33}$ machen deutlich, dass keine eindeutige Antwort auf diese Frage möglich ist, da die Ergebnisse jeweils stark von den zugrunde liegenden Annahmen abhängen. ${ }^{34}$ Eine Übertragung der Ergebnisse auf die reale Situation im europäischen Arzneimittelmarkt ist zudem unmöglich, da sich die Arbeiten auf die Betrachtung von wenigen Ländern beschränken müssen, die sich nur in wenigen Parametern unterscheiden. Die Europäische Union vereint dagegen 25 Mitgliedstaaten, die sowohl im Arzneimittelmarkt als auch bei allgemeinen wirtschaftlichen Größen wie z.B. dem Pro-Kopf-Einkommen wesentliche Unterschiede aufweisen. Auch wenn man die europäischen Länden danach aufteilt, ob sie Parallelimporteure oder -exporteure sind, erhält man zwei immer noch sehr heterogene Gruppen.

Eine aktuelle Studie versucht darum, die Auswirkungen von Arzneimittelparallelimporten in Europa auf die verschiedenen Akteure des Gesundheitswesens empirisch zu ermitteln und kommt dabei zu überraschenden Ergebnissen. ${ }^{35}$ Obwohl aufgrund der Preisdifferenzen in Europa ein großes Potential für die Senkung der Arzneimittelkos-

\footnotetext{
${ }^{30} \mathrm{Vgl}$. Breyer/Zweifel/Kifmann (2005), S. 473. Unter bestimmten Bedingungen (freier Marktzutritt, Möglichkeit der Preisdiskriminierung, keine Kreuz(preis)elastizitäten, keine strategische Interaktion der Firmen) ergibt sich bei monopolistischer Konkurrenz mit freiem Markteintritt auch ohne staatliche Eingriffe langfristig eine Preisstruktur, die den optimalen Ramsey-Preisen nahe kommt. Vgl. Danzon (1997b), S. 308f.

${ }^{31}$ In diesem Fall kann nicht ausgeschlossen werden, dass ein einheitlicher Preis die Wohlfahrt erhöht d.h. dass der Parallelhandel eine positive Wirkung hat -, auch wenn diese bei Ramsey-Preisen noch höher wăre. Vgl. Danzon (1998), Fußnote 2, S. 300.

${ }^{32} \mathrm{Vgl}$. Danzon (1998), S. 299 sowie die dort angegebene Literatur.

${ }^{33} \mathrm{Vgl}$. Kanavos/Costa-Font (2005), S. 756ff. sowie die dort angegebene Literatur.

${ }^{34}$ Unter bestimmten Annahmen ergibt sich sogar ein positiver Effekt von Parallelimporten auf die Gesamtwohlfahrt, ohne dass die Innovationsanreize der Hersteller abnehmen. Vgl. Königbauer (2004). ${ }^{35} \mathrm{Vgl}$. Kanavos/Costa-Font (2005). Betrachtet werden die europäischen Länder mit hohen Anteilen an Parallelimporten: Norwegen, Deutschland, Schweden, Dänemark, Großbritannien und die Niederlande.
} 
ten durch Parallelimporte besteht, sind die tatsächlich realisierten Einsparungen der Krankenkassen und Patienten sowohl in absoluten Werten als auch in Prozent der Gesamtausgaben für verschreibungspflichtige Medikamente für die betrachteten Länder eher gering. Die Produzenten dagegen erleiden jedoch finanzielle Einbußen, die sich auf die Rentabilität ihrer Forschungsarbeit auswirken können. Die eigentlichen Gewinner sind damit die Großhändler und Parallelimporteure sowie die Hersteller, die an der Distributionskette beteiligt sind. Die Autoren führen die geringen Einspareffekte bei Krankenkassen und Patienten darauf zurück, dass die nationalen Regulierungen diese oftmals verhindern, z.B. indem „Listenpreise“ erstattet werden, die große Gewinne bei den Zwischenhändlern ermöglichen.

Zudem sprechen die Ergebnisse der Studie dafür, dass Parallelimporte, anders als erwartet, keinen signifikanten Einfluss auf die Arzneimittelpreise im Importland haben. Diese sind vielmehr beeinflusst durch andere nationale Maßnahmen, wie z.B. Preisregulierungen oder die Förderung von Generika. Im Exportland können intensive Exporte bestimmter Arzneimittel zur Knappheit dieser Medikamente führen, da Großhändler einen Anreiz haben, große Mengen an Parallelimporteur zu liefern: Zum einen ermöglichen große internationale Preisdifferenzen Gewinne, die die im Heimatland realisierbaren übersteigen und zum anderen senkt die Lieferung an einen einzelnen Zwischenhändler statt an eine Vielzahl von Apotheken die Distributionskosten des Großhändlers deutlich.

Für die Autoren der Studie stellt sich darum die Frage, ob die geringen Kostensenkungen, die den nationalen Gesundheitssystemen zugute kommen, nicht auch durch andere Maßnahmen, wie beispielsweise Vereinbarungen mit der heimischen Industrie, erreicht werden könnten, die zudem die negativen Auswirkungen auf die Ertragslage der forschenden Hersteller senken würden. Es ist nicht zu erwarten, dass die Europäischen Institutionen ihre positive Sicht auf pharmazeutische Parallelimporte in naher Zukunft ändern werden, allerdings ist davon auszugehen, das letztlich die nationalen Regierungen mit den entsprechenden Regulierungsmaßnahmen einen großen Einfluss auf den Anteil der parallelimportieren Arzneimittel am Arzneimittelmarkt ausüben. Es bleibt daher ihnen überlassen, ob sie im Sinne einer Förderung der heimischen forschenden Industrie Parallelimporte eher durch andere Maßnahmen ersetzen oder aber durch entsprechende Änderungen der derzeitigen Regelungen dafür sorgen, dass die entstehenden Gewinne vorrangig den Versicherten und Patienten zugute kommen und nicht fast ausschließlich den Zwischenhändlern zufließen.

Somit stellt sich die Frage, ob die staatliche Förderung von Parallelimporten langfristig eine sinnvolle Strategie darstellt. Während in zahlreichen anderen Bereichen des $\mathrm{Ge}-$ sundheitswesens ein zunehmender Wettbewerb durch grenzüberschreitende Nachfrageverschiebungen wünschenswert erscheint, muss eine intensive Förderung von Parallelimporten in Europa aufgrund des Konfliktes zwischen dem Versuch der Kostendämpfung und industriepolitischen Interessen kritisch gesehen werden. Im Hinblick auf die Anreize der pharmazeutischen Hersteller zur Forschung und Entwicklung ist es ökonomisch sinnvoller, statt durch Parallelimporte einen Preiswettbewerb innerhalb der Patentlaufzeit zu initiieren, diesen durch die Förderung von Generika nach Ablauf der Schutzfrist zu intensivieren. $\mathrm{Da}$ in den vergangenen Jahrzehnten alle europäischen 
Staaten dazu gezwungen waren, Maßnahmen zur Dämpfung der Arzneimittelkosten zu ergreifen, hat auch die Förderung des Generikaeinsatzes an Bedeutung gewonnen, wodurch die Generikaindustrie in Europa gestärkt wurde. Darüber hinaus hat auch die Europäische Union verschiedene Maßnahmen ergriffen, um den Einsatz von Nachahmerpräparaten zu fördern.

\subsection{Generikaförderung}

Als Generikum bezeichnet man die Nachahmung eines bereits unter einem Markennamen im Markt befindlichen Arzneimittels, das eine im Wesentlichen wirkstoffgleiche Kopie des Originals darstellt, wobei die Hilfsstoffe und die Herstellungstechnologie variieren können. ${ }^{36}$ Generika dürfen nach Ablauf des Patentschutzes des Originalpräparats auf den Markt gebracht werden. Nach Richtlinie 2001/83/EG können sie nach einem verkürzten Verfahren zugelassen werden, wenn sie dem bereits erprobten Original im Wesentlichen gleichen. ${ }^{37} \mathrm{Da}$ bei ihrer Entwicklung Aufwendungen für Forschung und Entwicklung weitgehend entfallen, sind sie i.d.R. preisgünstiger als die Originalprodukte und stehen mit diesen im Preiswettbewerb. In diesem Sinne erfüllen Generikahersteller grundsätzlich eine wichtige ökonomische Aufgabe innerhalb des Gesundheitswesens, da sie einen Preiswettbewerb nach Ablauf des Patentschutzes initiieren - zu einem Zeitpunkt also, zu dem annahmegemäß die Kosten für Forschung und Entwicklung amortisiert sind und ein Preisaufschlag auf die Grenzkosten ökonomisch nicht mehr zu rechtfertigen ist. ${ }^{38}$ Auf diese Weise tragen sie auch zu einer Begrenzung der Arzneimittelausgaben bei.

Um die niedrigeren Preise für eine Senkung der Arzneimittelausgaben der Krankenversicherungsträger zu nutzen, existieren in den EU-Mitgliedstaaten verschiedene Regelungen, die Ärzten, Apothekern und Patienten Anreize zur Substitution von Originalprodukten durch preisgünstigere Generika geben.

Abbildung 4 zeigt, dass der Umsatzanteil der Generika am gesamten Arzneimittelmarkt im internationalen Vergleich stark variiert. Im Jahre 2001 wies dabei Deutschland mit 27 Prozent den höchsten Anteil unter den aufgeführten Ländern auf. Mit Großbritannien und Deutschland liegen damit im europäischen Vergleich zwei Länder an der Spitze, die eine relativ liberale Preissetzungspolitik für patentgeschützte Arzneimittel ermöglichen. Hier besteht aufgrund der im europäischen Vergleich relativ hohen Preise für diese Medikamente ein größerer Anreiz für die Generikahersteller, in den Markt einzutreten. Dies führt dazu, dass in Ländern mit restriktiveren Preisregulie-

\footnotetext{
${ }^{36}$ Das Europäische Parlament definiert in der Richtlinie 2001/83/EG Art. 10 Abs. 2 a) (mit den Änderungen durch Richtlinie 2004/27/EG) den Begriff „Generikum“ als „ein Arzneimittel, das die gleiche qualitative und quantitative Zusammensetzung aus Wirkstoffen und die gleiche Darreichungsform wie das Referenzarzneimittel aufweist und dessen Bioäquivalenz mit dem Referenzarzneimittel durch geeignete Bioverfügbarkeitsstudien nachgewiesen wurde."

${ }^{37}$ Vgl. Art. 10 Richtlinie 2001/83/EG des Europäischen Parlaments und des Rates vom 6. November 2001

zur Schaffung eines Gemeinschaftskodexes für Humanarzneimittel.

${ }^{38}$ Vgl. Danzon/Chao (2000), S. 312.
} 
rungen für patentierte Arzneimittel nach dem Ablauf des Patentschutzes der Generikawettbewerb geringer ausfällt und diese Länder darum im internationalen Vergleich höhere Preise für patentfreie Originalarzneimittel aufweisen. ${ }^{39}$ Der Sachverständigenrat zur Begutachtung der Entwicklung im Gesundheitswesen $(2005)^{40}$ geht davon aus, dass Deutschland mit Umsatzanteilen der Generika von 68 Prozent am generikafähigen Markt und 30 Prozent am Gesamtmarkt im Jahre 2003 den weltweit größten Marktanteil von Generikapräparaten besaß.

Abbildung 4: Umsatzanteile der Generika an den nationalen Arzneimittelmärkte 2001 in Prozent

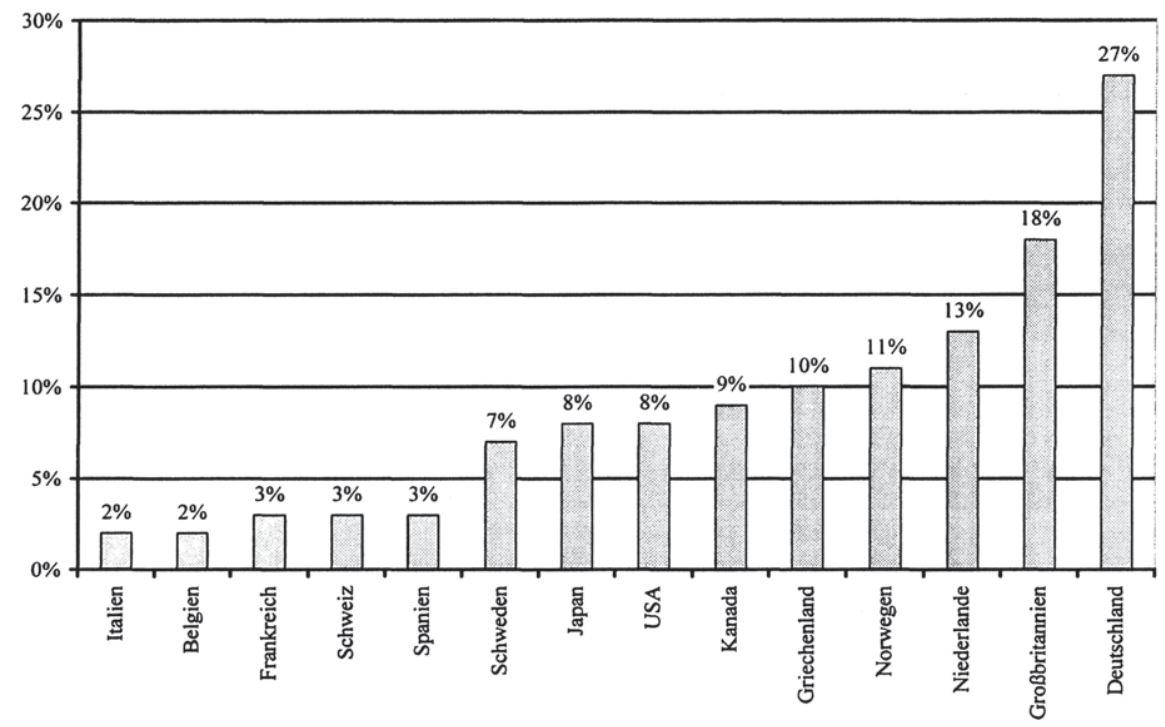

Quelle: Europäische Kommission (2003f), S. 57

Die Kostendämpfungsmaßnahmen, die seit Mitte der 70er Jahre zur Begrenzung der Arzneimittelausgaben beitragen sollten, führten zu einer strukturellen Verschiebung am Arzneimittelmarkt zugunsten von Generika, deren Anteil am generikafähigen Markt bereits ab Anfang der 70er Jahre zu steigen begann. ${ }^{41}$ Tabelle 10 zeigt eine Übersicht möglicher Maßnahmen zur Förderung der Nachfrage nach Generika bei den beteiligten Akteuren.

Bereits seit 1993 gab es Bestrebungen der EU, den Einsatz von Generika zu fördern. Das EU-Parlament ersuchte im November 1993 die Kommission, entsprechende Maß-

${ }^{39} \mathrm{Vgl}$. Danzon/Furukawa (2003), S. 526.

${ }^{40} \mathrm{Vgl}$. Sachverständigenrat zur Begutachtung der Entwicklung im Gesundheitswesen (2005), S. 41, Ziffer 34 und S. 722 , Ziffer 941.

${ }^{41}$ Vgl. Mehnert/Wille/Rohweder (1994), S. $9 f$. 
nahmen zu prüfen. ${ }^{42}$ Auch die Mitteilung der Kommission über den Binnenmarkt für Arzneimittel vom 25.11.1998 enthielt Forderungen bezüglich der Förderung eines Wettbewerbs durch Generika, die aufgrund der alleinigen Zuständigkeit der Mitgliedstaaten für die Ausgestaltung ihrer Sozialversicherungssysteme jedoch rein deklaratorischen Charakter haben.

Tabelle 10: Maßnahmen zur Förderung der Generikanachfrage

\begin{tabular}{|l|l|}
\hline Ärzte & $\begin{array}{l}\text { Arzneimittelbudget } \\
\text { Rückmeldung über Verordnungsverhalten }\end{array}$ \\
\hline Apotheker & Generikasubstitution \\
\hline Konsumenten & $\begin{array}{l}\text { Zuzahlungen (in Abhängigkeit vom Arzneimittel- } \\
\text { preis) } \\
\text { Festbetrags- bzw. Referenzpreissystem } \\
\text { Informationspolitik }\end{array}$ \\
\hline
\end{tabular}

Quelle: Rosian/Vogler/Weigl (2000), S. 32

Bedeutendere Auswirkungen infolge expliziter rechtlicher Regelungen ergaben sich für die Generikahersteller in den vergangenen Jahren insbesondere durch die Revision des europäischen Arzneimittelrechts im Jahre 2004. Ausgangspunkt für diese Änderung ist die Mitteilung der EU-Kommission „Die pharmazeutische Industrie Europas zum Wohle der Patienten stärken "“43 aus dem Jahre 2003, die in Reaktion auf den Bericht der G-10-Arzneimittelgruppe „Innovation und Bereitstellung von Arzneimitteln“ einige Vorschläge zur Stärkung der Wettbewerbsfähigkeit der europäischen Pharmaindustrie enthält. ${ }^{44}$ Dabei empfiehlt die Kommission unter anderem die Entwicklung eines wettbewerbsfähigen Generikamarktes, insbesondere um eine nachhaltige Finanzierung der Arzneimittelversorgung in den Mitgliedstaaten zu erreichen. Als Leitaktionen zur Erreichung dieses Ziels werden genannt: ${ }^{45}$

- die Einführung der sog. Roche-Bolar-Regelung für Generika, die es deren Herstellern erlaubt, die erforderlichen Versuche und Arbeiten für die Zulassung bereits während der Patentlaufzeit des Originalpräparats durchzuführen, um eine schnelle Markteinführung der Generika zu ermöglichen,

- die Einführung eines Zulassungsantrags für Generika und die Erteilung der Zulassung während der letzten zwei Jahre der Datenschutzfrist für das Originalprodukts, ${ }^{46}$

\footnotetext{
${ }^{42} \mathrm{Vgl}$. Rosian/Vogler/Weigl (2000), S. 19.

${ }^{43}$ Europäische Kommission (2003b).

${ }^{44} \mathrm{Vgl}$. Europăische Kommission (2003b), S. 14ff.

${ }^{45} \mathrm{Vgl}$. Europäische Kommission (2003b), S. 20.

${ }^{46}$ Eine Ausnahme sollten hier Arzneimittel bilden, für die das zentralisierte Verfahren vorgeschrieben ist. Dieses Verfahren ist seit der Revision der europäischen pharmazeutischen Gesetzgebung im März 2004 für Arzneimittel mit neuen Stoffen obligatorisch, wenn sie zur Behandlung von Aids, Krebs, Diabetes und neurodegenerativen oder seltenen Erkrankungen bestimmt sind.
} 
- eine klarere Definition für Generika in der EU,

- die Einfuhrung flexiblerer Regelungen, damit Generikahersteller ihre Produkte in Mitgliedstaaten vertreiben können, in denen das Referenzarzneimittel nicht auf dem Markt ist sowie

- die Behandlung der Frage biologisch vergleichbarer Produkte mit dem Ziel, die Herstellung von Kopien dieser Produkte durch die Einrichtung eines klaren Regulierungsrahmens zu ermöglichen.

Mit der Änderung der Richtlinie 2001/83/EG ${ }^{47}$ vom 31. März 2004 wurden diese Maßnahmen in europäisches Recht gefasst, das mit der 14. Novelle des AMG in nationales deutsches Recht Eingang fand. Die bedeutendste Änderung bezog sich auf den Unterlagenschutz, der einem Zweitantragsteller für eine bestimmte Frist nach der Marktzulassung des Originalarzneimittels verbietet, sich bei der Zulassung auf das vom Originalhersteller eingereichte Zulassungsdossier zu beziehen. Zuvor variierte die Dauer dieses Schutzes innerhalb der EU relativ stark. In Spanien und Irland betrug sie beispielsweise sechs Jahre, während in Großbritannien und Deutschland der Unterlagenschutz für 10 Jahre galt und in einigen der Beitragsstaaten ein solcher Schutz überhaupt nicht existierte. Für zentral zugelassene Arzneimittel galt generell eine zehnjährige Schutzfrist. Nun wurde der Unterlagenschutz EU-weit einheitlich auf acht Jahre festgelegt, d.h. dass damit küntig ein Zulassungsantrag für ein Generikum mit Bezug auf die Unterlagen des Originalherstellers bereits nach acht Jahren möglich ist. Die Vermarktung darf aber frühestens nach zehn Jahren erfolgen, sofern der Patentschutz bzw. das ergänzende Schutzzertifikat für das Originalpräparat dann bereits abgelaufen sind. Wird das Originalpräparat innerhalb der achtjährigen Schutzfrist für eine signifikant neue Indikation zugelassen, verlängert sich die Frist für die alleinige Vermarktung des Originalherstellers um ein weiteres Jahr auf insgesamt 11 Jahre. Zudem führte die Novelle des AMG die Roche-Bolar-Regelung in das Patentrecht ein, so dass nun Versuche und Studien, die für eine Marktzulassung notwendig sind, bereits während der letzten zwei Jahre der Patentlaufzeit erlaubt sind. Auf diese Weise kann die Markteinführung von Generika schneller nach dem Ablauf der Schutzfristen für das Originalprodukt erfolgen, so dass möglichst früh ein Preiswettbewerb einsetzt, der dazu beiträgt, die Arzneimittelausgaben der Krankenkassen einzudämmen. Die Umsetzung des europäischen Rechts führte darüber hinaus zu einer Änderung des Heilmittelwerbegesetzes, die den Herstellern einen größeren Spielraum in Bezug auf Informationen über nicht verschreibungspflichtige Arzneimittel einräumt und die Möglichkeiten der Werbung für Arzneimittel und Medizinprodukte außerhalb der Fachkreise erweitert. ${ }^{48} \mathrm{~Pa}$ ckungsbeilagen und Fachinformationen für die frei verkäuflichen OTC-Produkte unterliegen nicht mehr dem Heilmittelwerbegesetz. Darüber hinaus formulierte die Richtlinie erstmals gesetzliche Vorschriften für das Genehmigungsverfahren von biogeneri-

\footnotetext{
${ }^{47}$ Richtlinie 2001/83/EG vom 6.11.2001 zur Schaffung eines Gemeinschaftskodex für Humanarzneimittel.

${ }^{48} \mathrm{Vgl}$. Allgemeine Ortskrankenkasse (AOK) (2005d).
} 
schen Produkten ${ }^{49}$ und vereinfachte die Zulassung von Nachahmerprodukten in Ländern, in denen das Referenzarzneimittel nicht im Markt befindlich ist.

Auch die Rechtsprechung des EuGH trug zur Stärkung der Position der Generikahersteller bei. Durch sein Urteil vom 20. Januar 2005 in der Rechtssache „SmithKline Beecham" erleichterte der Gerichtshof die vereinfachte Zulassung von Nachahmerpräparaten. ${ }^{50}$ Im betreffenden Fall hatten zwei Arzneimittelhersteller die Zulassung für ein Generikum des Antidepressivums Seroxat in Dänemark beantragt. Dieses Generikum war mit dem Originalpräparat nicht vollständig identisch, sondern beinhaltete andere Salze als Hilfsstoffe, weshalb die dänische Zulassungsbehörde weitere Nachweise verlangte, um feststellen zu können, ob die Präparate ,im Wesentlichen“ gleich sind. Der Originalhersteller GlaxoSmithKline argumentierte nun, dass allein die Tatsache, dass zusätzliche Unterlagen für diesen Nachweis nötig seien, gegen diese Gleichheit spreche. Dieser Auffassung folgte der EuGH in seinem Urteil nicht und entschied, dass eine verkürzte Zulassung eines Generikums auch dann möglich sei, wenn aufgrund abweichender Hilfsstoffe zusätzliche Nachweise nötig seien, um die Gleichheit mit dem Original zu belegen. Zum Begriff der „wesentlichen Gleichheit“ hatte der EuGH bereits in der Rechtssache C-368/96 entschieden, dass ein Arzneimittel im Wesentlichen einem originalen Arzneimittel gleiche, wenn es die gleiche qualitative und quantitative Zusammensetzung der Wirkstoffe sowie die gleiche Bioverfügbarkeit ${ }^{51}$ aufweise und in der gleichen Darreichungsform erhältlich sei. ${ }^{52}$ Allerdings sei eine wesentliche Gleichheit nicht gegeben, wenn es nach dem Stand der Wissenschaft gegenüber dem Originalpräparat offensichtlich in Bezug auf Sicherheit und Wirksamkeit erhebliche Unterschiede gäbe. Dennoch sei es aber generell das Ziel der europäischen Arzneimittel-Richtlinie, „die Wiederholung von Versuchen bei Menschen und Tieren“ nach Möglichkeit zu vermeiden.

Neben den beschriebenen Einflüssen der Europäischen Union in Gestalt von Maßnahmen zur Erleichterung der Generikazulassung und durch die Rechtsprechung des EuGH existieren auch indirekte Wirkungen der Integration der europäischen Märkte auf die Generikahersteller. Hier spielen die Grundfreiheiten - und dabei insbesondere die Freiheit des Kapitalverkehrs sowie die Warenverkehrsfreiheit - eine große Rolle.

Die Freiheit des Kapitalverkehrs ermöglicht nach Artikel 56 des EGV allen EUBürgern und Drittstaatenangehörigen in Deutschland Investitionen zu tätigen, die keinen speziellen Beschränkungen und Diskriminierungen unterliegen dürfen. Diese Grundfreiheit ist seit der Gründung der EG im Vertrag verankert, wurde jedoch erst 1990 im Rahmen des EU-Binnenmarktes vollständig umgesetzt. Die Kapitalverkehrsfreiheit in Verbindung mit einer zunehmenden wirtschaftlichen Globalisierung führt $\mathrm{zu}$

\footnotetext{
${ }^{49}$ Biogenerika sind Nachbildungen biotechnologisch hergestellter Biopharmazeutika, welche zum Teil wiederum körpereigene Proteine nachahmen.

${ }^{50} \mathrm{Vgl}$. Europäischer Gerichtshof (2005).

${ }^{51}$ Die Bioverfügbarkeit bezeichnet den Freisetzungsgrad eines Wirkstoffanteils, der nach einer gewissen Zeitspanne unverăndert im Blutkreislauf nachzuweisen ist. Allgemeine Ortskrankenkasse (AOK) (2005c).

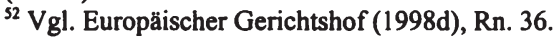


einer steigenden Integration der Volkswirtschaften im Bereich der grenzüberschreitenden Finanzströme. Im weltweiten Generikamarkt führten diese Entwicklungen zu zahlreichen Firmenübernahmen und -zusammenschlüssen, die zu einer zunehmenden Konzentration beitragen. Tabelle 11 zeigt exemplarisch einige Kăufe und Verkäufe der letzten Jahre. Auch für die Zukunft werden zahlreiche weitere Übernahmen erwartet, die zu einer größeren Konzentration im Generikamarkt führen werden. Der deutsche Markt gilt dabei aufgrund des hohen Generikaanteils und der guten Wachstumschancen als besonders interessant.

Tabelle 11: Übernahmen im Generikamarkt

\begin{tabular}{|l|l|}
\hline 2000 & $\begin{array}{l}\text { ratiopharm übernimmt Technilab (drittgrößter kanadischer Generika- } \\
\text { hersteller) }\end{array}$ \\
\hline 2002 & $\begin{array}{l}\text { ratiopharm erwirbt ribosepharm (deutscher Hersteller von onkologi- } \\
\text { schen Arzneimitteln) }\end{array}$ \\
\hline 2003 & $\begin{array}{l}\text { Pfizer (USA) kauft Pharmacia (Kanada) und übernimmt damit auch } \\
\text { deren Tochterfirma Heumann }\end{array}$ \\
\hline 2004 & $\begin{array}{l}\text { ratiopharm übernimmt Magnafarma (drittgrößter niederländischer Ge- } \\
\text { nerikahersteller) } \\
\text { britischer Finanzinvestor 3i kauft betapharm }\end{array}$ \\
\hline 2005 & $\begin{array}{l}\text { Novartis (Schweiz) übernimmt HEXAL und Eon Labs (USA) und } \\
\text { wird damit Weltmarktführer für Generika } \\
\text { Stada erwirbt die Vertriebsgesellschaft Ciclum Farma (Portugal) } \\
\text { Teva (Israel) kauft Ivax (USA) und übernimmt damit wieder die Füh- } \\
\text { rung im weltweiten Generikamarkt } \\
\text { Pfizer (USA) verkauft Heumann an Torrent Pharmaceutical (Indien) }\end{array}$ \\
\hline
\end{tabular}

Quelle: eigene Darstellung

Zusammenfassend ist festzustellen, dass der Markt für Nachahmerpräparate - vor allem aufgrund des politischen Interesses an einer Begrenzung der Arzneimittelausgaben - in der Vergangenheit signifikant an Bedeutung gewonnen hat und auch für die $\mathrm{Zu}$ kunft ein weiteres Wachstum zu erwarten ist. Inwieweit Änderungen im System wie beispielsweise die Möglichkeit direkter Preis-verhandlungen zwischen Herstellern und Krankenkassen das Sinken der Wettbewerbsintensität aufgrund der sich abzeichnenden Konzentrationsbewegungen im Markt aufhalten oder verhindern können, bleibt abzuwarten.

Insgesamt betrachtet ergeben sich für die deutschen Generikahersteller durch den Einfluss der EU und die Marktintegration tendenziell eher positive Einflüsse. Die Veränderungen im europäischen Arzneimittelrecht aus dem Jahre 2004 brachten ihnen mehr Rechtssicherheit bei der Vermarktung ihrer Produkte auf den verschiedenen europäischen Märkten und die Möglichkeit einer schnelleren Markteinführung nach dem Ablauf des Patentschutzes der Originalpräparate. Diese Änderung, die durch die Aufnah- 
me der Roche-Bolar-Regelung möglich wurde, trägt dazu bei, dass diejenigen Länder der EU, die diese Regelung bisher nicht kannten - darunter auch Deutschland -, im Standortwettbewerb für die Generikahersteller an Attraktivität gewinnen.

Die Wirkung der Kapitalverkehrsfreiheit in Verbindung mit einer zunehmenden wirtschaftlichen Globalisierung lässt bereits in der näheren Zukunft zahlreiche Eigentümerwechsel im Generikamarkt erwarten, die sowohl die mittelständischen Hersteller als auch - wie das Beispiel HEXAL zeigt - Firmen mit großen Marktanteilen betreffen. Umgekehrt gibt diese Entwicklung deutschen Firmen die Möglichkeit, in lukrativen ausländischen Märkten zu investieren - eine Möglichkeit, die überwiegend von den finanzstarken Branchenführern genutzt werden kann.

Die Freiheit des Warenverkehrs und die damit verbundene Möglichkeit des Re- und Parallelimports von Arzneimitteln betreffen die Generikahersteller aufgrund des geringeren Preisniveaus ihrer Produkte in wesentlich geringerem Maße als die forschenden Pharmafirmen. Sollten internationale Preisdifferenzen im Generikamarkt dennoch in erheblichem Maß zu Importen und damit zu Umsatzeinbußen der Hersteller führen, bestehen für die Hersteller bessere Möglichkeiten, diese Differenzen durch eine entsprechende Preissetzung zu reduzieren, weil Generika in den meisten europäischen Ländern weniger restriktiven Preisregulierungen unterworfen sind als die patentgeschützten Originale. Ohnehin ist dieser Anreiz bei patentgeschützten Originalpräparaten durch das höhere Preisniveau und die Preisdifferenzen infolge der national unterschiedlichen Regulierungsdichte wesentlich höher, so dass der Import vorwiegend bei diesen Arzneimitteln interessant ist.

Die günstige Position der Generikahersteller in Deutschland und Europa wird durch die Finanzierungsschwierigkeiien der europäischen Sozialversicherungssysteme gestärkt, die dazu führen, dass die Förderung der Generikasubstitution in vielen europäischen Ländern als Mittel zur Begrenzung der Arzneimittelausgaben genutzt wird. In diesem Zusammenhang können die Generikahersteller eine positive Entwicklung erwarten, die durch politische Maßnahmen auf nationaler und europäischer Ebene unterstützt wird. 


\subsubsection{Auswirkungen einer steigenden Patientenmobilität auf die Arz- neimittelhersteller}

Die „Kohll/Decker"-Rechtsprechung des EuGH bietet den Versicherten und Patienten die Möglichkeit, neben ärztlichen (Dienst-)Leistungen auch bestimmte Waren im Ausland zu Lasten ihrer Krankenversicherung nachzufragen. Die Warenver-kehrsfreiheit ermöglicht neben dem gewerblichen Parallelimport damit auch eine private grenzüberschreitende Nachfrage nach Arzneimitteln. Die Bedeutung von Arzneimitteln in der Gesundheitsversorgung der Bevölkerung zeigt sich auch in den Zahlen zu den von der GKV getragenen Arzneimittelausgaben. Von der GKV erstattete Arzneimittel verursachten in den Jahren 1998 bis 2004 zwischen 14,8 und 16,6 Prozent der gesamten Leistungsausgaben der GKV. Damit waren sie regelmäßig der drittgrößte Ausgabenblock hinter den Ausgaben für Krankenhausbehandlungen und ambulanten ärztlichen Leistungen und nahmen im Jahre 2004 hinter den stationären Leistungen sogar den zweiten Platz ein. ${ }^{53}$

Im Gegensatz zu ärztlichen Leistungen stellen Arzneimittel sehr homogene Güter dar, die aufgrund verschiedener Regulierungen auf europäischer Ebene europaweit in vergleichbarer Qualităt angeboten werden. Dadurch entfallen weitgehend die Qualitătsunsicherheiten, die bei anderen Leistungen ein bedeutendes Hindernis für eine grenzüberschreitende Nachfrage darstellen. Zudem können viele Arzneimittel per Post versandt werden, wodurch sich die Nachfrager anders als beim Dienstleistungsimport nicht ins Ausland begeben müssen, um vorhandene Preisunterschiede zu nutzen. Aufgrund dieser Eigenschaften ist eine Verschiebung der privaten Nachfrage ins Ausland in nennenswertem Ausmaß bei Arzneimitteln wahrscheinlicher als in anderen Bereichen des Gesundheitswesens. Wichtige Determinanten einer solchen Nachfrageverschiebung sind die international bestehenden Preisunterschiede, die insbesondere für den Selbstmedikationsmarkt von Bedeutung sind, sowie die Ausgestaltung der im Inland anfallenden Zuzahlungen, die bestimmt, inwieweit Versicherte einen Anreiz besitzen, Rezepte zu Lasten der GKV bei ausländischen Apotheken einzureichen. In diesem Zusammenhang ist auch das Entstehen deutschsprachiger Angebote ausländischer Internetapotheken von Bedeutung, die Arzneimittel auf Rezept an deutsche Versicherte versenden dürfen. ${ }^{54}$

Während die Generikahersteller vom Phänomen der gewerblichen Parallelimporte von Arzneimitteln in deutlich geringem Maße betroffen sind als die forschenden Hersteller, da das Preisniveau ihrer Produkte wesentlich geringer ist und somit internationale Preisunterschiede i.d.R. zu wesentlich geringeren absoluten Preisabständen führen, spielt die Unterscheidung zwischen Generika und Originalpräparaten für den privaten Import von Arzneimitteln eine eher untergeordnete Rolle. Im Folgenden wird vielmehr zwischen rezeptpflichtigen und rezeptfreien Produkten unterschieden, weil sich durch

\footnotetext{
${ }^{53} \mathrm{Vgl}$. Bundesministerium für Gesundheit (BMG) (2005), Tabelle 16.6.A.

${ }^{54}$ Eine ausfuhrliche Darstellung der deutschen Zuzahlungsregelungen und der Angebote ausländischer Versandapotheken erfolgt erst in Abschnitt 3.3.2.2, der die Betrachtung des Apothekenmarktes zum Inhalt hat. Soweit diese Entwicklungen im vorliegenden Abschnitt Relevanz besitzen, werden sie aber bereits hier kurz erläutert und in die Überlegungen einbezogen.
} 
die divergierenden Regulierungen für diese beiden Gruppen in Deutschland für die Versicherten unterschiedliche Anreize zum grenzüberschreitenden Einkauf bieten.

Durch den privaten Einkauf von Arzneimitteln haben die deutschen Hersteller nur dann Nachteile zu erwarten, wenn die Kunden überwiegend Produkte ausländischer Hersteller wählen oder wenn die Herstellerabgabepreise ihrer Produkte im Ausland z.B. aufgrund staatlicher Regulierungen - geringer sind als in Deutschland. Es ist davon auszugehen, dass der größte Teil dieser privaten Arzneimittelimporte in Form einer Bestellung bei ausländischen Versandapotheken erfolgt. Die bekannteste ausländische Versandapotheke ist die holländische Apotheke DocMorris. Weitere deutschsprachige Angebote existieren in Holland, Tschechien und Großbritannien. Derzeit ist der Marktanteil dieser Anbieter am gesamten Arzneimittelmarkt noch sehr gering. ${ }^{55}$ Auswirkungen auf die Ertragssituation der Hersteller würden sich darum nur dann ergeben, wenn sie ihre Produkte dort aufgrund staatlicher Regulierungen zu Preisen anbieten müssten, die deutlich unter den deutschen Apothekenabgabepreisen liegen.

Wie gerade ausgeführt, ist für die Ertragssituation der Hersteller der Vergleich der Herstellerabgabepreise entscheidend. Hierbei muss zwischen Arzneimitteln für den Selbstmedikationsbereich und rezeptpflichtigen Arzneimitteln unterschieden werden. So ist im Selbstmedikationsbereich die Preisbildung in vielen europäischen Ländern liberalisiert, so auch in den Niederlanden und in Großbritannien. ${ }^{56}$ Zudem existieren keine staatlichen Reglementierungen bezüglich der Distributionszuschläge von OTCProdukten. ${ }^{57}$ Die Hersteller haben demnach einen direkten Einfluss auf die Preissetzung und können selbst entscheiden, inwieweit sie ihre Preissetzung zwischen den verschiedenen Ländern variieren. Sie müssen keine Rückwirkungen staatlicher Regulierungen auf die einheimische Nachfrage beachten. Eine Ausnahme bildet dagegen Tschechien, wo alle im Markt befindlichen Arzneimittel staatlichen Preiskontrollen unterliegen. $\mathrm{Da}$ aufgrund des deutlich geringeren Pro-Kopf-Einkommens zu erwarten ist, dass die Preisregulierungen tendenziell zu geringeren Arzneimittelpreisen führen als in Deutschland, dürfte der finanzielle Anreiz für die Patienten, Arzneimittel in Tschechien zu kaufen, größer sein als sie bei den Apotheken in den Niederlanden oder in Großbritannien zu erwerben. Da jedoch der Preis nicht das alleinige Entscheidungskriterium beim Arzneimittelimport darstellt und die tschechische Versandapotheke derzeit nur über ein sehr begrenztes Angebot verfugt, ist für die nähere Zukunft keine spürbare Nachfrageverschiebung dorthin zu erwarten. Zudem bleibt allgemein für den OTC-Bereich zu beachten, dass mit dem GMG seit dem 1.1.2004 auch in Deutschland eine freie Preissetzung ohne Regulierungen auf der Groß- und Einzelhandelsebene möglich ist, die zu einem verstärkten Preiswettbewerb unter den deutschen Apotheken

\footnotetext{
5s Der Bundesverband deutscher Versandapotheken (BVDVA) schătzt, dass ihr Anteil am Arzneimittelumsatz 2004 zwischen 0,5 und 1,5 Prozent lag. Vgl. Lahm (2005).

${ }^{56}$ Vgl. Association Européene des Spécialités Pharmaceutiques Grand Public (AESGP) (2004a), S. 10. In Großbritannien müssen die Hersteller für OTC-Produkte lediglich einen Mindestverkaufspreis festlegen, der im Handel nicht unterschritten werden darf. Vgl. Österreichisches Bundesinstitut fur Gesundheitswesen (ÖBIG) (Hrsg.) (1998), S. 95.

${ }^{57} \mathrm{Vgl}$. Beratungsgesellschaft für Angewandte Systemforschung (BASYS)/Infras (2002), S. 220; Association Européene des Spécialités Pharmaceutiques Grand Public (AESGP) (2004b), S. 19.
} 
geführt hat. Der Gründer der holländischen Versandapotheke DocMorris erklärte in einem Interview, dass diese Entwicklung den Vertrieb von OTC-Produkten für seine Firma weitgehend unattraktiv mache. ${ }^{58}$

Bei den - in der Regel teureren - rezeptpflichtigen Arzneimitteln, die in Deutschland der Preisbindung der zweiten Hand unterliegen - profitieren die ausländischen Apotheken jedoch von der Möglichkeit, günstigere Zuzahlungsbedingungen und Rabatte anbieten zu können. ${ }^{59}$ Für die Hersteller ist auch hier entscheidend, inwieweit sich die Herstellerabgabepreise im Ausland von den deutschen unterscheiden. In den Niederlanden dürfen die Apothekenabgabepreise erstattungsfähiger Arzneimittel einen bestimmten maximalen Level nicht überschreiten. ${ }^{60}$ Großbritannien hat im Sommer 2005 ein neues Erstattungssystem für Generika eingeführt, die Preissetzung der Hersteller bleibt aber grundsătzlich frei. ${ }^{61}$ Spürbare Auswirkungen auf die Ertragssituation der deutschen Arzneimittelindustrie können sich jedoch generell nur dann ergeben, wenn ausländische Regulierungen zu deutlich geringeren Herstellerabgabepreisen führen. Aus der Beobachtung, dass rezeptpflichtige Arzneimittel in anderen EU-Ländern niedrigere Apothekenabgabepreise aufweisen, kann jedoch nicht direkt auf die Höhe der dort erzielbaren Herstellerabgabepreise geschlossen werden. Berücksichtigt werden müssen vielmehr auch die Höhe der Groß- und Einzelhandelsspannen sowie die Umsatzsteuerbelastung.

Tabelle 12 vergleicht für ausgewählte europäische Länder die Anteile der Komponenten Herstellerabgabepreis, Handelsspannen und Umsatzsteuer an den Apothekenverkaufspreisen für erstattungsfähige Arzneimittel. Dabei zeigt sich, dass in Deutschland der Anteil des Herstellerabgabepreises am Apothekenverkaufspreis im europäischen Vergleich relativ gering ist. Deshalb ist es möglich, dass ein Arzneimittel trotz eines höheren Herstellerabgabepreises im Ausland für die Patienten und Krankenkassen letztendlich günstiger ist. Eine Modellrechnung verdeutlicht, dass ein solches Szenario für erstattungsfähige Arzneimittel in den Niederlanden nicht unrealistisch ist. Unter der Annahme, dass ein Arzneimittel in den Niederlanden einen um 15 Prozent geringeren Apothekenverkaufspreis aufweist als in Deutschland, ${ }^{62}$ ergibt sich für ein Arzneimittel, dass in Deutschland $100 €$ kostet, in den Niederlanden ein Apothekenpreis von $85 €$. Unter Zugrundelegung der Anteile aus Tabelle 12 errechnet sich daraus ein Herstellerabgabepreis von 53,72 $€$ in den Niederlanden, während er in Deutschland lediglich 49,90€ beträgt. Für Großbritannien stellt sich die Situation aufgrund des im internationalen Vergleich sehr hohen Anteils des Herstellerabgabepreises ähnlich dar. Unter diesen Bedingungen entstünde den Generikaherstellern kein Nachteil, wenn deut-

\footnotetext{
${ }^{58}$ Vgl. o.V. (2005b).

${ }^{59} \mathrm{Vgl}$. furr eine ausführlichere Darstellung Abschnitt 3.3.2.2.

${ }^{60} \mathrm{Vgl}$. Rychlik/Bundesverband der Pharmazeutischen Industrie (BPI) (2003), S. 21.

${ }^{61} \mathrm{Vgl}$. Department of Health (2005).

${ }^{62}$ Diese Annahme folgt einer Angabe des Oberlandesgerichts Hamm, das in einem Urteil darüber, ob die niederländische Internetapotheke DocMorris Arzneimittel zu Preisen anbieten darf, die unterhalb der deutschen Apothekenabgabepreisen liegen, davon ausging, dass die um durchschnittlich 15 Prozent geringeren Preise von DocMorris dem niederländischen Preisniveau entsprechen. Vgl. o.V. (2004), S. A 2650.
} 
sche Patienten ihre Produkte verstärkt in diesen Ländern nachfragen würden. Auf Basis der verfügbaren Informationen ist darum keine Aussage darüber möglich, wie eine signifikante Nachfrageverschiebung ins Ausland aus Sicht der Hersteller von Generika zu bewerten ist.

Tabelle 12: Anteile des Herstellerabgabepreises, der Vertriebskosten und der Umsatzsteuer am Apothekenverkaufspreis (AVP) im EU-Vergleich ${ }^{63}$

\begin{tabular}{|l|l|l|l|}
\hline & $\begin{array}{l}\text { Anteiliger } \\
\text { Herstellerab- } \\
\text { gabepreis am } \\
\text { AVP (brutto) }\end{array}$ & $\begin{array}{l}\text { Gesamtspanne } \\
\text { des Handels } \\
\text { in Prozent des } \\
\text { AVP (brutto) }\end{array}$ & $\begin{array}{l}\text { Anteil der Um- } \\
\text { satzsteuer am } \\
\text { AVP (brutto) }\end{array}$ \\
\hline Dänemark & 49,2 & 25,8 & 25,0 \\
\hline Deutschland & 49,9 & 34,1 & 16,0 \\
\hline Belgien & 56,4 & 37,6 & 6,0 \\
\hline Luxemburg & 57,4 & 39,6 & 3,0 \\
\hline Österreich & 62,1 & 37,9 & 0,0 \\
\hline Spanien & 62,6 & 33,4 & 4,0 \\
\hline Griechenland & 62,1 & 28,9 & 9,0 \\
\hline Finnland & 62,9 & 29,1 & 8,0 \\
\hline Niederlande & 63,2 & 30,8 & 6,0 \\
\hline Italien & 63,2 & 26,8 & 10,0 \\
\hline Frankreich & 66,9 & 31,0 & 2,1 \\
\hline Portugal & 67,4 & 27,2 & 5,4 \\
\hline Großbritannien & 72,4 & 27,6 & 0,0 \\
\hline Schweden & 77,6 & 22,4 & 0,0 \\
\hline
\end{tabular}

Quelle: Glaeske et al. (2003); Österreichisches Bundesinstitut für Gesundheitswesen (ÖBIG) (Hrsg.) (2001); eigene Berechnungen

\subsubsection{Apotheken}

Als Distributionsstellen für Arzneimittel spielen die öffentlichen Apotheken in der Gesundheitsversorgung der Bevölkerung eine wichtige Rolle. Dabei unterliegt dieser Bereich zahlreichen Regulierungen, die auf zwei grundsätzlichen gesundheitspoliti-

${ }^{63}$ In Frankreich, Großbritannien (ausgenommen Generika), Italien und in den Niederlanden bezieht sich die Gesamtspanne des Handels auf den erstattungsfähigen Arzneimittelmarkt, in Portugal auf rezeptpflichtige Arzneimittel und in allen anderen Ländern auf den gesamten Arzneimittelmarkt. 
schen Zielen beruhen, an denen sich die Arzneimittelversorgung in Deutschland orientiert. $^{64}$

Eines dieser Ziele stellt die Sicherung der Arzneimittelversorgung in räumlicher, quantitativer, qualitativer und zeitlicher Hinsicht dar. ${ }^{65}$ Der Patient soll an jedem Ort Deutschlands die benötigten Arzneimittel in hoher Qualität zu jeder Tageszeit ohne unzumutbare Wartezeiten erhalten. Aus diesem Ziel leitet sich die Forderung nach einer flächendeckenden Versorgung mit Abgabestellen für rezeptpflichtige Arzneimitteln und einem bundesweit einheitlichen Apothekenabgabepreis ab.

Darüber hinaus haben die staatlichen Eingriffe in das Vertriebssystem von Arzneimitteln das Ziel, die Bevölkerung vor den Gefahren des Arzneimittelkonsums zu schützen. ${ }^{66}$ Der Patient als medizinischer Laie sieht sich häufig selbst nicht in der Lage, seine Krankheit richtig zu diagnostizieren und die angemessene Dosierung der Arzneimittel einzuschätzen und kann sich durch einen unsachgemäßen Gebrauch selbst schädigen. Zudem besteht die Gefahr, dass Arzneimittel im Übermaß konsumiert werden (Arzneimittelmissbrauch). Um die Verbraucher vor diesen Risiken zu schützen, beschränkt sich die Abgabe vieler Arzneimittel auf Apotheken (Apothekenpflicht). ${ }^{67} \mathrm{Be}-$ steht auch bei bestimmungsgemäßem Gebrauch eine Gefahr für den Menschen, wenn Arzneimittel ohne ärztliche Überwachung eingenommen werden, so kann der Bundesminister für Gesundheit diese Produkte zusätzlich in die Verschreibungspflicht aufnehmen. ${ }^{68}$ Sie dürfen dann nur nach Vorlage einer Verschreibung durch einen Arzt an die Verbraucher abgegeben werden. Eine Selbstbedienung ist bei diesen Medikamenten nicht zulässig. ${ }^{69}$ Die Abgabe von apothekenpflichtigen Arzneimitteln bleibt ausschließlich entsprechend ausgebildeten Personen überlassen. ${ }^{70}$ Bezüglich der personellen Ausstattung unterliegen Apotheken zahlreichen Regulierungen, die dazu führen, dass der Apothekensektor eine Personalstruktur aufweist, die deutlich von der des übrigen Einzelhandels abweicht. Im Durchschnitt sind die Beschäftigten besser qualifiziert, wodurch sich Auswirkungen auf die Kostenstruktur ergeben. Wer eine Apotheke betreiben möchte, benötigt eine behördliche Erlaubnis. ${ }^{71}$ Diese Erlaubnis knüpft das Gesetz über das Apothekenwesen (ApoG) an bestimmte Bedingungen. Hierzu zählen die Approbation als Apotheker oder eine vergleichbare Qualifikation eines anderen Landes. ${ }^{72}$ Dieses sog. Fremdbesitzverbot untersagt Nicht-Apothekern die Führung ei-

\footnotetext{
${ }^{64}$ Neben öffentlichen Apotheken existieren in Deutschland Krankenhausapotheken, die jedoch bisher nur für die Versorgung der Klinikpatienten mit Medikamenten zuständig sind und keine Arzneimittel an die Öffentlichkeit abgeben dürfen. Im Folgenden wird auf diese nicht năher eingegangen. Wenn von Apotheken die Rede ist, sind offentliche Apotheken gemeint.

${ }^{65} \mathrm{Vgl}$. Schöffski (1995), S. 35f.

${ }^{66}$ Vgl. Schoffski (1995), S. 37f.

${ }^{67} \mathrm{Vgl}$. § 43 AMG.

${ }^{68} \mathrm{Vgl}$. 48 Gesetz über den Verkehr mit Arzneimitteln (AMG).

${ }^{69} \mathrm{Vgl}$. $\S 17$ Abs. 3 Verordnung über den Betrieb von Apotheken (ApBetrO) und $\S 52$ Abs. 1 Gesetz über das Apothekenwesen (ApoG).

${ }^{70} \mathrm{Vgl}$. 3 Abs. 4 und 5 ApBetrO.

"Vgl. § 1 Abs. 2 ApoG.

${ }^{72} \mathrm{Vgl}$. $§ 2$ Abs. 1 Satz 3 ApoG und $\S 2$ Abs. 2 ApoG in Verbindung mit Anlage I.
} 
ner Apotheke. ${ }^{73}$ Die Erlaubnis verpflichtet den Apotheker zur persönlichen und eigenverantwortlichen Leitung der Apotheke. ${ }^{74}$ Darüber hinaus ist die Bildung von größeren Apothekenketten in Deutschland nicht möglich. Das GMG hob das generelle Mehrbesitzverbot von Apotheken zwar auf, weiterhin ist einem Apotheker aber lediglich der Betrieb von maximal vier Apotheken gestattet. Die behördliche Erlaubnis bezieht sich ausschließlich auf den darin genannten Apotheker und die aufgeführten Räume. Diese Räume unterliegen bestimmten Mindestanforderungen. ${ }^{75}$ Der Vertrieb von Arzneimitteln wurde mit dem GMG liberalisiert, so dass seit dem Jahr 2004 ein Versandhandel mit Medikamenten erlaubt ist.

Abbildung 5: Vergleich der Apothekenspannen vor und nach dem GMG (in $€)^{76}$

Apothekenspanne

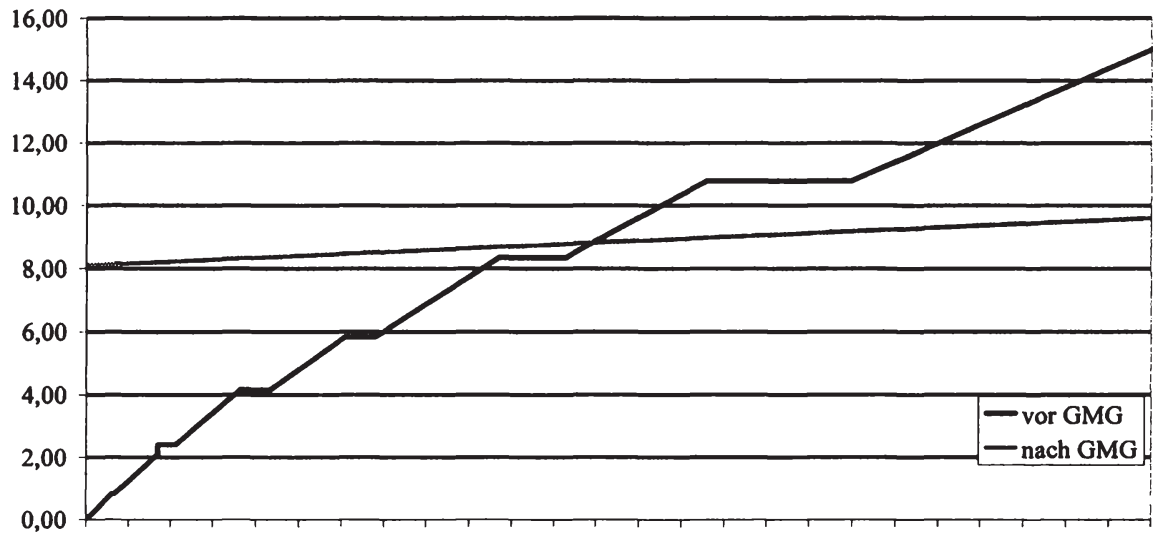

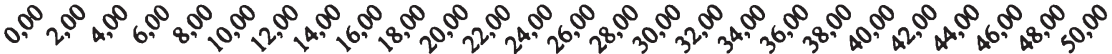

Apothekeneinkaufspreis

Quelle: AMPreisV; eigene Berechnungen

${ }^{73}$ Auch eine Beteiligung von Nicht-Apothekern ist nicht möglich, da jeder Gesellschafter Inhaber einer Erlaubnis sein muss. Die Bildung einer Stillen Gesellschaft mit Nicht-Apothekern ist untersagt. Vgl. § 8 ApoG.

${ }^{74} \mathrm{Vgl}$. $\$ 7$ ApoG. Wird die Apotheke als Filialapotheke von einem angestellten Apotheker gefürt, so hat dieser ebenfalls die Verpflichtungen, die im ApoG und der ApBetrO fur Apothekenleiter festgelegt sind, zu erfüllen. Vgl. § 2 Abs. 5 Satz 2 ApoG.

${ }^{75}$ So muss die Apotheke mindestens aus einer Offizin (Verkaufsraum), einem Laboratorium, einem Nachtdienstzimmer und ausreichendem Lagerraum mit einer Lagertemperatur von unter $20^{\circ} \mathrm{C}$ bestehen. Die Grundfläche der Betriebsräume darf nicht kleiner sein als $110 \mathrm{~m}^{2}$. Darüber hinaus bestehen zahlreiche weitere Regelungen beispielsweise in Bezug auf die Einrichtung der Răume und die Ausstattung mit Gerăten.

${ }^{76}$ Aus Praktikabilitätsgründen wird hier nur der Verlauf bis zu einem Apothekeneinkaufspreis von 50 $€$ dargestellt. Im weiteren Verlauf stieg die Spanne vor dem GMG mit der dargestellten Steigung bis zu einem Apothekeneinkaufspreis von 543,92 $€$ und verlief dann etwas flacher. Die Neuregelung fuhrt zu einer gleich bleibenden Steigung über den gesamten Preisbereich. 
Im Jahre 2005 erzielten die deutschen Apotheken 72,9 Prozent ihres Umsatzes mit verschreibungspflichtigen Arzneimitteln. Für diese Medikamente gelten bundeseinheitliche Apothekenabgabepreise, die durch die in der Arzneimittelpreisverordnung (AMPreisV) geregelten Spannen für Großhandel und Apotheken erreicht werden. Das GMG änderte die Apothekenspanne von einer prozentualen, degressiven Spanne in ein fixes Abgabehonorar von 8,10€ pro verschriebenem Medikament und einen Zuschlag von 3 Prozent pro Packung. Abbildung 5 zeigt einen Vergleich zwischen alter und neuer Apothekenspanne in Abhängigkeit vom Apothekeneinkaufspreis ${ }^{77}$.

Wie aus der Graphik zu erkennen ist, sank durch die Neuregelung der Anreiz für die Apotheker, bevorzugt teure Medikamente abzugeben. Darüber hinaus änderte sich die Preisstruktur der verschreibungspflichtigen Arzneimittel. Während sich relativ günstige Arzneimittel zu gegebenen Apothekeneinkaufspreisen verteuerten, wurden Medikamente mit einem Apothekeneinkaufspreis über 23,82 $€$ günstiger.

Diese Preisregulierung wird zum einen damit begründet, dass sie die Patienten vor überhöhten Preisen schützen soll. ${ }^{78} \mathrm{Da}$ die Zahlungsbereitschaft der Patienten stark mit ihrem Gesundheitszustand variiert und eine schwere Erkrankung häufig mit einer besonderen Dringlichkeit der Nachfrage einhergeht, verhindere die Regulierung, dass der Apotheker diese Notlage durch überhöhte Preisforderungen ausnutzen kann. Zudem will man den Patienten nicht zumuten, im Krankheitsfall Preisvergleiche anzustellen, um den günstigsten Anbieter zu finden. Da die Konsumenten durch den hohen Grad des Krankenversicherungsschutzes und die über weite Bereiche fixe Zuzahlung für Arzneimittel $^{79}$ den Apothekenverkaufspreis nicht selbst tragen müssen, ist diese Begründung für einen staatlichen Eingriff in die Preisbildung jedoch kaum haltbar. Von Bedeutung ist diese Regulierung vielmehr für die Arzneimittelausgaben der Krankenkassen. ${ }^{80}$ Aufgrund der preisunelastischen Nachfrage ließen sich Preisforderungen der Apotheker gegenüber den Verbrauchern leicht durchsetzen, so dass die Krankenversicherungen und damit über höhere Beiträge die Gesamtheit der Beitragszahler belastet würden. Eine Begrenzung der Apothekenabgabepreise stellt somit ein Mittel zur Kostendämpfung dar.

Auf der anderen Seite soll die Preisgestaltung für Arzneimittel aber auch dazu beitragen, eine ordnungsgemäße Arzneimittelversorgung der Bevölkerung zu gewährleisten, indem sie die Existenzgrundlage einer ausreichenden Zahl von Apotheken sichert. ${ }^{81}$ Es besteht die Befürchtung, dass eine Freigabe der Preise zu einem Verdrängungswettbewerb führen würde, der insbesondere Apotheken in dünn besiedelten, ländlichen Ge-

\footnotetext{
${ }^{77}$ Basis fur die Berechnung ist der Netto-Apothekeneinkaufspreis.

${ }^{78} \mathrm{Vgl}$. Schöffski (1995), S. 39f.

${ }^{79}$ Vgl. zur Ausgestaltung der deutschen Zuzahlungsregelungen Abschnitt 3.3.2.2.

${ }^{80}$ Die Apotheken erzielten 77,2 Prozent des Apothekenumsatzes im Jahre 2005 mit Medikamenten, die zu Lasten der gesetzlichen bzw. privaten Krankenkassen oder sonstiger Kostenträger verordnet wurden (55,5 Prozent aller abgegebenen Packungen). Vgl. Bundesvereinigung Deutscher Apothekerverbånde (ABDA) (2006), S. 46.

${ }^{81}$ Vgl. im Folgenden Schöfski (1995), S. 42f. Wie im nachfolgenden Abschnitt erläutert wird, existiert kein Richtwert, der vorgibt, wie groß die Zahl der Apotheken sein muss, um eine ausreichende Arzneimittelversorgung zu garantieren.
} 
bieten betrifft, da die Einwohnerzahl dort möglicherweise zu gering ist, um die Rentabilität einer im freien Wettbewerb stehenden Apotheke zu sichern. Aus dieser Perspektive zielt das Preissystem aber darauf ab, dass "auch die letzte zur Versorgung der Bevölkerung als notwendig erachtete Apotheke damit überleben kann". ${ }^{82}$ Demnach zielt die Preisverordnung darauf ab, das Überleben anderweitig unrentabler Apotheken zu sichern. Daraus ergibt sich, dass Apotheken in günstigen Lagen Gewinne in einer GröBenordnung erzielen können, wie sie im Wettbewerb nicht realisierbar wären. Deshalb ist davon auszugehen, dass der tatsächliche Apothekenabgabepreis in diesen Apotheken oberhalb des Wettbewerbspreises liegt. Es erscheint darum fragwürdig, ob die beiden oben genannten Ziele mit einem einheitlichen Apothekenabgabepreis gleichzeitig realisierbar sind. Vielmehr ist davon auszugehen, dass der einheitliche Apothekenabgabepreis Ineffizienzen schafft, die aus ökonomischer Sicht eine Änderung der Preisverordnung dringend erforderlich machen. Eine denkbare Lösung wäre eine Höchstpreisverordnung, wie sie in den Niederlanden angewandt wird. Auf diese Weise ließe sich der Schutz der Bevölkerung vor überhöhten Preisen sicherstellen ${ }^{831}$ und zugleich würde man den Apothekern einen größeren Anreiz zu wirtschaftlichem Verhalten geben, was letztlich den Konsumenten und Beitragszahlern zugute käme.

Seit dem GMG gelten der einheitliche Apothekenabgabepreis und die neue Spannenregelung nur noch für verschreibungspflichtige Arzneimittel. Rezeptfreie Medikamente sind aus der Preisbindung entlassen und können grundsätzlich nicht mehr von den gesetzlichen Krankenkassen erstattet werden. ${ }^{84}$ Hier ist deshalb ein Preiswettbewerb der Apotheken möglich, von dem die Patienten direkt profitieren, da sie die Kosten vollständig selbst tragen müssen.

Nach Berechnungen des Österreichischen Bundesinstituts für Gesundheitswesen (ÖBIG) aus dem Jahre 1999 wies Deutschland mit 31,7 Prozent im europäischen Vergleich nach Luxemburg die zweithöchste Apothekenspanne und mit 40,6 Prozent ebenfalls die zweithöchste Gesamthandelsspanne ${ }^{85}$ (jeweils in Prozent des Apothekenverkaufspreises) auf. ${ }^{86}$ Verschiedene gesetzliche Maßnahmen während der letzten Jahre z.B. durch das Arzneimittelausgaben-Begrenzungsgesetz (AABG) und das Beitragssicherungsgesetz (BSSichG) führten zwar zu einem Absinken der Gesamthandelsspanne auf 37,3 Prozent; gleichwohl lag Deutschland damit weiterhin im Vorderfeld der Vergleichsländer. ${ }^{87}$ Allerdings blieben bei dieser Berechnung die Effekte der Spannenänderungen durch das GMG unberücksichtigt, die zu einer Verringerung der Distributionskosten für Arzneimittel um mindestens 700 Mio. $€$ geführt haben sollen. ${ }^{88}$

\footnotetext{
${ }^{82}$ Schöffski (1995), S. 43.

${ }^{83} \mathrm{Vgl}$. Schöffski (1995), S. 42.

${ }^{84}$ Von dieser Regelung existieren Ausnahmen für Kinder bis 12 Jahre und Jugendliche mit Entwicklungsverzögerungen bis 18 Jahre. Für die zu Lasten der GKV abgegebenen rezeptfreien Arzneimittel gilt weiterhin die alte Spannenregelung.

${ }^{85}$ Eine Handesspanne entspricht generell der Differenz zwischen dem Einkaufspreis und dem Verkaufspreis (ohne $\mathrm{MwSt}$ ) eines Produkts.

${ }^{86} \mathrm{Vgl}$. Österreichisches Bundesinstitut für Gesundheitswesen (ÖBIG) (Hrsg.) (2001).

${ }^{87} \mathrm{Vgl}$. Glaeske et al. (2003), S. 20ff.

${ }^{88} \mathrm{Vgl}$. Wasem/Greß/Niebuhr (2005), S. 29.
} 
Da zudem keine aktuellen Studien zu Änderungen in den Spannen der Vergleichsländer verfuggar sind, ist eine Aussage über die derzeitige Position Deutschlands im internationalen Vergleich nicht möglich.

\subsubsection{Situation auf dem Apothekenmarkt}

Das Verbot des Fremdbesitzes und die nur eingeschränkte Möglichkeit zum Mehrbesitz machen die mittelständische Apotheke zur Regel auf dem deutschen Apothekenmarkt. Im Jahre 2005 existierten in Deutschland 21.476 Apotheken, so dass eine Apotheke durchschnittlich 3.842 Einwohner vorsorgte. Nach einem Rückgang der Apothekenzahl in den Jahren 2001 bis 2003 stieg sie in den Jahren 2004 und 2005 wieder an, blieb jedoch hinter ihrem Höchststand aus dem Jahre 2000 (21.592 Apotheken) zurück (vgl. Abbildung 6).

Es fällt schwer zu quantifizieren, wie hoch der Versorgungsgrad mindestens sein muss, um eine ordnungsgemäße Versorgung mit Arzneimitteln zu gewährleisten. Die Klage, die letztendlich dazu führte, dass das Bundesverfassungsgericht im Jahre 1958 die Bedarfsplanung bei Apotheken für nicht rechtmäßig erklärte, ging von einem Apotheker aus, dem die Eröffnung einer neuen Apotheke mit der Begründung verwehrt wurde, dass die rund 6.000 Einwohner der relevanten Gemeinde durch die bestehende Apotheke ausreichend versorgt seien. ${ }^{89}$ Für eine weitere Apotheke fehle die Existenzgrundlage. Die zuständige Behörde ging davon aus, dass 7.000 bis 8.000 Einwohner pro Apotheke notwendig seien, um deren wirtschaftliche Existenz zu sichern. Heute liegen die entsprechenden durchschnittlichen Werte in Deutschland erheblich unter dieser damals angestrebten Zahl. In den westdeutschen Bundesländern lagen sie im Jahre 2003 zwischen 3.020 und 3.870 Einwohnern je Apotheke und auch im Osten Deutschlands versorgte eine Apotheke durchschnittlich in keinem Bundesland nirgendwo mehr als 5.000 Einwohner. ${ }^{90}$ Der Sachverständigenrat für die Konzertierte Aktion im Gesundheitswesen beurteilte bereits im Jahre 1992 die Ausstattung der neuen Bundesländer mit Abgabestellen für Arzneimittel als ausreichend. ${ }^{91}$ Danach stieg die Zahl der Apotheken in Ostdeutschland weiter an, von 2.187 im Jahre 1992 auf $3.281 \mathrm{im}$ Jahre 2002. ${ }^{92}$

Im internationalen Vergleich lag Deutschland bezüglich der Einwohnerzahl pro Apotheke im Jahre 1996 im Mittelfeld der europäischen Staaten (vgl. Abbildung 7). Die geringste Apothekendichte wiesen die Niederlande auf, die höchste Griechenland. Die durchschnittliche Apothekendichte betrug für die hier aufgeführten

\footnotetext{
${ }^{89} \mathrm{Vgl}$. Bundesverfassungsgericht (Hrsg.) (1958), S. 381.

${ }^{90} \mathrm{Vgl}$. Bundesvereinigung Deutscher Apothekerverbände (ABDA) (2004), S. 35.

${ }^{11} \mathrm{Vgl}$. SVRKAiG (1992), S. 65.

${ }^{92}$ Nach 2002 erhob die ABDA keine getrennten Zahlen mehr für die ostdeutschen Bundesländer.
} 
Länder 3.280 Einwohner pro Apotheke. Deutschland hatte demnach mit 3.847 Einwohnern pro Apotheke eine leicht unterdurchschnittliche Apothekendichte. ${ }^{93}$

Abbildung 6: Entwicklung der Apothekenzahl

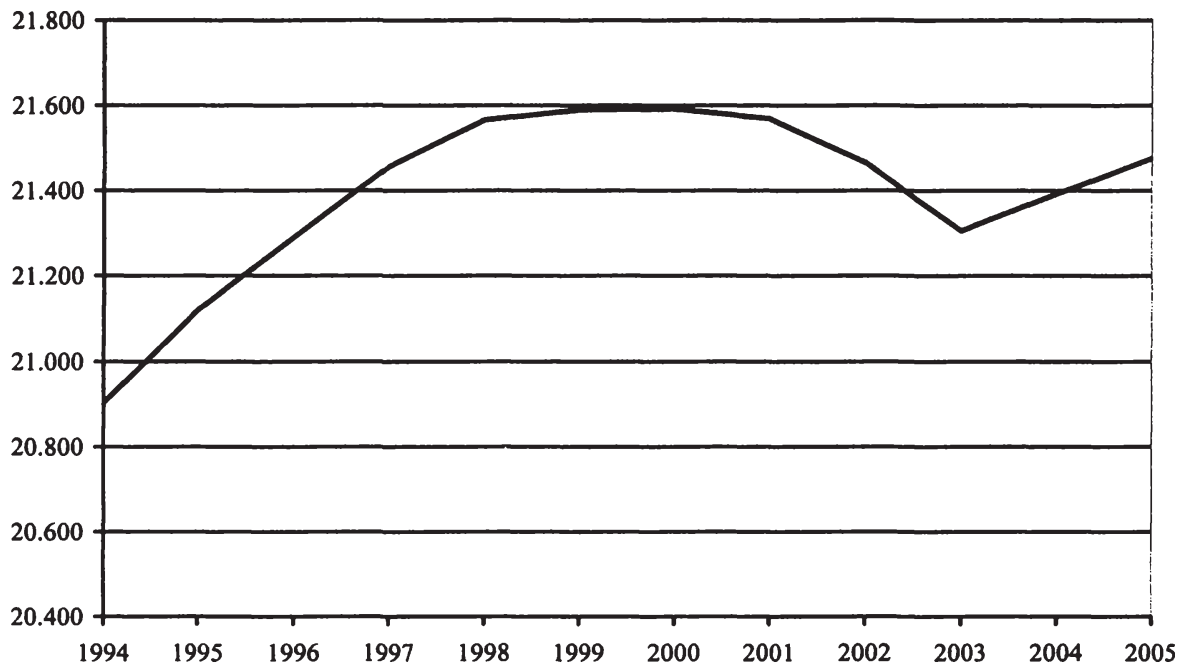

Quelle: Bundesvereinigung Deutscher Apothekerverbände (ABDA) (2006), S. 42; eigene Darstellung

Obwohl sich die Zahl der Einwohner je Apotheke nur bedingt als Indikator für die Güte der Arzneimittelversorgung eignet, legen die dargestellten Daten den Schluss nahe, dass ein leichtes Absinken der Apothekendichte in Deutschland die flächendeckende Versorgung der Bevölkerung mit Arzneimitteln nicht generell gefährden würde.

Derzeit existiert im pharmazeutischen Einzelhandel aufgrund der zahlreichen Regulierungen eine polypolistische Marktstruktur. ${ }^{94}$ Sowohl auf der Angebots- als auch auf der Nachfrageseite stehen sich zahlreiche Marktteilnehmer gegenüber, dennoch können insbesondere in ländlichen Gegenden durch die untergleichmäßige räumliche Verteilung der Apotheken regionale Monopole oder Oligopole existieren. Doch auch in Gebieten mit hoher Apothekendichte herrscht im Unterschied zum theoretischen Ideal

${ }^{93}$ Bezieht man weitere Abgabestellen für Arzneimittel mit ein, wie beispielsweise selbstdispensierende Ärzte, verändert sich das Bild nur leicht. Weiterhin zeigt sich der höchste Versorgungsgrad in Griechenland (1.282 Einw./Abgabestelle) und der geringste in den Niederlanden (7.104 Einw./Abgabestelle). Im Durchschnitt ergibt sich eine Zahl von 2.983 Einwohnern pro Abgabestelle für Arzneimittel. Auch hier weist Deutschland, das neben öffentlichen Apotheken keine weiteren Abgabestellen für Arzneimittel zulässt, eine überdurchschnittliche Dichte auf.

${ }_{94} \mathrm{Vgl}$. im Folgenden Schöffski (1995), S. $169 \mathrm{ff}$. 
eines polypolistischen Marktes lediglich ein eingeschränkter Wettbewerb, denn im Bereich der apothekenpflichtigen Arzneimittel ${ }^{95}$ haben die Apotheken auf Grund ihres Vertriebsmonopols keine Konkurrenz durch andere Einzelhändler zu befürchten. Innerhalb dieses Segments fällt zudem der Preis als Wettbewerbsparameter aus, so dass eine Konkurrenz hier nur im Rahmen anderer Parameter (Service, Ausstattung, Sortiment etc.) stattfinden kann.

Abbildung 7: Einwohner pro öffentliche Apotheke 1996, internationaler Vergleich

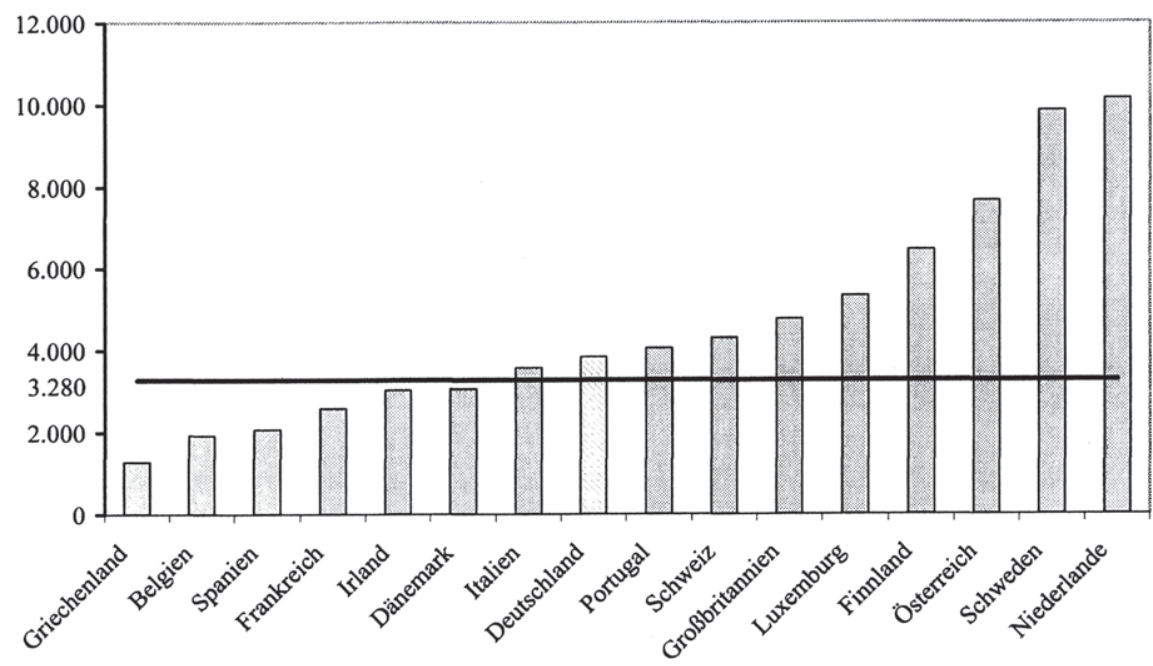

Quelle: Österreichisches Bundesinstitut für Gesundheitswesen (ÖBIG) (Hrsg.) (1998); eigene Berechnungen

Im Nebensortiment (2005: 1,2 Mrd. €; 3,4 Prozent des Umsatzes) und im Bereich der frei verkäuflichen Arzneimittel (2005: 1,0 Mrd. €; 2,9 Prozent des Umsatzes) besteht eine Wettbewerbssituation zwischen Apotheken und anderen Einzelhandelsgeschäften (z.B. mit Drogerien, aber auch Supermärkten und Discountern) ${ }^{96}$ Hier besitzt der „normale“ Einzelhandel deutliche Vorteile, da er weniger von Regulierungen betroffen ist und darum eine andere Kostenstruktur aufweist. Es existieren weniger restriktive Regelungen beispielsweise bezüglich Personal, Selbstbedienung, Preissetzung und Werbung. Dennoch fällt der Umsatz des normalen Einzelhandels mit freiverkäuflichen

${ }^{95}$ Im Jahre 2005 trugen die apothekenpflichtigen Arzneimittel (inkl. verschreibungspflichtige und nicht-verschreibungspflichtige) 31,4 Mrd. $€$, d.h. 89,7 Prozent zum Umsatz bei. Vgl. Bundesvereinigung Deutscher Apothekerverbände (ABDA) (2006), S. 46, eigene Berechnungen.

${ }_{96} \mathrm{Vgl}$. zu den Daten Bundesvereinigung Deutscher Apothekerverbände (ABDA) (2006), S. 46. 
Arzneimitteln geringer aus als der Apothekenumsatz in diesem Segment. ${ }^{97}$ Dies kann zum Teil darauf zurückzufuihren sein, dass trotz fehlender gesetzlicher Preisregulierungen ein Großteil der Produkte im Nebensortiment der Apotheken einer faktischen Preisbindung durch unverbindliche Preisempfehlungen der Hersteller unterliegt. ${ }^{98}$ Diese Produkte werden oftmals ausschließlich über Apotheken vertrieben. Bietet ein Apotheker diese Produkte über längere Zeit und systematisch zu einem geringeren Preis an, wird der Hersteller, wenn er die Exklusivität der Produkte erhalten will, diese nicht mehr über die betreffende Apotheke vertreiben. Es ist daher davon auszugehen, dass diese Warengruppen innerhalb der Apotheken durchschnittlich deutlich höhere Preise aufweisen als im sonstigen Einzelhandel. Vom der Höhe des Umsatzes kann insofern nicht auf die abgesetzte Menge an freiverkäuflichen Arzneimitteln im ,normalen“ Einzelhandel geschlossen werden. Es ist jedoch nicht auszuschließen, dass auch die Menge der abgesetzten freiverkäuflichen Arzneimittel in Apotheken höher ausfällt als im „normalen“ Einzelhandel, wenn die Kunden zum einen bei Arzneimittel aus Apotheken eine höhere Qualität unterstellen und zum anderen den Einkauf von frei verkäuflichen Arzneimitteln gewöhnlich mit der Einlösung von Rezepten in Apotheken verbinden.

$\mathrm{Da}$ in weiten Bereichen des Apothekensortiments kein Preiswettbewerb stattfindet, kann sich der Wettbewerb nur in anderen Parametern wie Service oder Ausstattung der Apotheke niederschlagen. Insgesamt war das Verhältnis der Apotheker untereinander in den letzten Jahrzehnten jedoch eher von Kollegialität als von Konkurrenz geprägt, ${ }^{99}$ so dass die Wettbewerbsintensität auch in diesem Bereich relativ gering ausfällt. Eine größere Konkurrenz besteht seit der Preisfreigabe im Jahre 2004 im Bereich der apothekenpflichtigen aber nicht verschreibungspflichtigen Arzneimittel, deren Anteil am Gesamtumsatz der Apotheken 2005 bei 16,8 Prozent lag.

Es bestehen darum hinreichende Gründe für die Annahme, dass aufgrund der zahlreichen Regulierungen im gesamten deutschen Gesundheitswesen die derzeitige Marktsituation im Apothekensektor nicht das Effizienzziel erreicht und auch nicht den Präferenzen der Konsumenten entspricht. Die eingeschränkte Konsumentensouveränität verbietet zwar eine völlige Liberalisierung des Apothekenmarktes, gleichwohl wären jedoch innerhalb der bestehenden Grenzen Schritte in Richtung einer Deregulierung wünschenswert.

$\mathrm{Zu}$ einer teilweisen Liberalisierung des Apothekenbesitzes kam es durch das GMG im Jahre 2004. Seither ist es Apothekern erlaubt, neben einer Hauptapotheke bis zu drei Filialapotheken zu besitzen, deren Leitung (angestellte) Apotheker übernehmen müssen. Nach Erhebungen der ABDA existierten zwei Jahre nach der Freigabe des be-

\footnotetext{
${ }^{97}$ Im Jahre 2005 wurden außerhalb der Apotheken freiverkäufliche Arzneimittel im Wert von 0,4 Mrd. $€$ umgesetzt. Der Apothekenumsatz in diesem Segment betrug 1,0 Mrd. $€$. Vgl. Bundesvereinigung Deutscher Apothekerverbände (ABDA) (2006), S. 46.

${ }^{98} \mathrm{Vgl}$. Schöffski (1995), S. 170.

${ }^{99}$ Die Apotheker sind bereits durch ihre Berufsordnungen zu kollegialem Verhalten verpflichtet. Siehe z.B. Berufsordnung für Apotheker des Landes Niedersachsen (1993) vom 19. November 1993, §5: "Der Apotheker ist verpflichtet, sich gegenüber den Angehörigen seines Berufes kollegial zu verhalten."
} 
schränkten Mehrbesitzes in Deutschland 1.425 Filialapotheken. ${ }^{100}$ Das entspricht einem Anteil von 6,6 Prozent an allen Apotheken. In 80 Prozent der Fälle betreibt ein Apotheker neben der Hauptapotheke eine einzige Tochterapotheke. Lediglich 17 Apotheker besitzen bisher die gesetzlich festgelegte Höchstzahl von vier Apotheken. Damit ist bisher keine dynamische Entwicklung hin zu Apothekenketten zu beobachten. Dies legt den Schluss nahe, dass zumindest kleine Ketten bei der derzeitigen Situation in Deutschland ökonomisch kaum rentabel sind. Dafür können - neben der relativ kurzen Zeit, die seit der Änderung vergangen ist - verschiedene Gründe verantwortlich sein. Da das Verbot des Fremdbesitzes bisher aufrecht erhalten bleibt, können lediglich Apotheker Ketten betreiben. Sie verfügen als typische Mittelständler jedoch nicht über das wirtschaftliche Potential, in kurzer Zeit eine bedeutende Zahl von Apotheken zu

übernehmen bzw. zu eröffnen. Zudem führt die Eröffnung von Filialapotheken nur beschränkt zu Kostenvorteilen. Zwar steigt durch eine zusätzliche Apotheke der Gesamtumsatz, doch durch die geltenden Regelungen bezüglich des Personals und der Räumlichkeiten, die für jede einzelne Apotheke gelten, ist keine wesentliche Degression der Fixkosten möglich. Kostenvorteile können sich für den Betreiber lediglich beim Einkauf der Produkte ergeben. Die in der AMPreisV geregelten Großhandelsspannen sind als Höchstspannen ausgestaltet. Da sich die Apothekenspanne am Höchstwert orientiert, legt die Preisverordnung den Apothekenabgabepreis de facto fest. Bleibt der Großhändler mit seinen Preisforderungen unter dem möglichen Höchstwert, so kommt die Differenz dem Apotheker zu Gute. Ein größeres Bestellvolumen ermöglicht es dann, höhere Rabatte vom Großhandel zu erhalten. Doch insgesamt wird sich die Gewinnsituation durch die Eröffnung zusätzlicher Apotheken nicht gravierend verbessern. So ist zu erwarten, dass unter den derzeit geltenden gesetzlichen Bestimmungen die Zahl der Apothekenketten weiterhin nur moderat ansteigen wird.

Die Bildung von Apothekenketten in Deutschland könnte sich jedoch beschleunigen, wenn es zu einer generellen Aufgabe des Mehrbesitz- sowie des Fremdbesitzverbots käme. Ohnehin stellt sich die Frage, ob nach der Aufgabe des Mehrbesitzverbotes das Verbot des Fremdbesitzes unter medizinisch-pharmakologischen und/oder ökonomischen Gründen noch zu rechtfertigen ist. Da durch das GMG die Leitung einer Apotheke auch durch einen angestellten Apotheker möglich ist und damit die medizinische Verantwortung für den Betrieb vom Besitz der Apotheke unabhängig wurde, entfällt die medizinisch-pharmakologische Begründung, warum einem Nicht-Apotheker der Besitz einer Apotheke verwehrt bleiben soll. Zur Sicherung der Qualität der Arzneimittelabgabe und -herstellung ist es nicht notwendig, dass der Besitzer Apotheker ist. Es reicht aus, wenn die Leitung einer Apotheke durch einen Apotheker erfolgt, der während der Öffnungszeiten anwesend ist. Die Freigabe des Mehrbesitzes zieht sachlogisch die Aufhebung des Fremdbesitzverbots nach sich. In diesem Sinne fordert auch der Sachverständigenrat für die Begutachtung der Entwicklung im Gesundheitswesen

${ }^{100} \mathrm{Vgl}$. Hollstein (2006). 
in seinem Gutachten aus dem Jahre 2005 eine mittelfristige Aufgabe des Mehr- und Fremdbesitzverbots, um die Wettbewerbsintensität im Apothekensektor zu erhöhen. ${ }^{101}$

In Bezug auf den Fremdbesitz von Apotheken könnten sich in Zukunft durch die europäische Rechtsprechung Veränderungen ergeben. Ende des Jahres 2005 leitete die Europäische Kommission ein Vertragsverletzungsverfahren ${ }^{102}$ gegen Italien ein, weil nach ihrer Auffassung die dortigen Regelungen zum Fremdverbot bei privaten Apotheken gegen die europäische Niederlassungsfreiheit bzw. die Kapitalverkehrsfreiheit verstoßen. ${ }^{103}$ Eine entsprechende Klage beim EuGH reichte sie im Juni 2006 ein. Die italienischen Regelungen sehen u.a. vor, dass nur Apotheker oder juristische Personen, die aus Apotheken bestehen, öffentliche Apotheken besitzen dürfen. $\mathrm{Da}$ auf diese Weise Wirtschaftsteilnehmer - auch aus anderen Mitgliedstaaten -, die keine Apotheker sind, daran gehindert werden, sich an öffentlichen Apotheken zu beteiligen oder solche zu eröffnen, sind diese Regelungen nur dann mit europäischem Recht vereinbar, wenn sie aus Gründen des Allgemeininteressens zu rechtfertigen sind. Die italienischen Behörden begründen dieses Verbot mit dem Schutz der öffentlichen Gesundheit. Nach Auffassung der Kommission ist das Verbot für die Erreichung dieses Zieles jedoch nicht zwingend notwendig, da es zur Qualitätssicherung beispielsweise genügen würde, für die Medikamentenausgabe an Patienten und die Bestandsverwaltung die Anwesenheit eines Apothekers vorzuschreiben. Zudem kennt das italienische Recht bereits eine Ausnahme von der genannten Regelung, weil Familienmitglieder eines verstorbenen Apothekers dessen Apotheke bis zu zehn Jahre weiterführen dürfen, auch wenn sie nicht Apotheker sind. Sollte der EuGH entscheiden, dass die genannten Regelungen gegen europäisches Recht verstoßen, ergäbe sich damit auch ein Änderungsbedarf der deutschen Vorschriften.

Auch die Eröffnung einer Filialapotheke im Juli 2006 in Saarbrücken durch die Versandapotheke DocMorris hat zu entsprechenden Diskussionen in der deutschen Apothekenlandschaft und Gesundheitspolitik gefuihrt. ${ }^{104}$ Besitzer dieser Apotheke ist eine Kapitalgesellschaft, deren Gründer Däinghaus zudem kein Apotheker ist. Damit liegt hier ein Verstoß gegen das Fremdbesitzverbot vor. Däinghaus beruft sich jedoch darauf, dass das deutsche Fremdbesitzverbot gegen die europäische Niederlassungsfreiheit verstoße und darum die Eröffnung der Apotheke in Saarbrücken mit europäischem Recht vereinbar sei. Da rechtliche Schritte der konkurrierenden Apotheker zu erwarten sind, ist auch in diesem Fall eine abschließende Entscheidung des EuGH wahrscheinlich.

Die Aufhebung des Mehr- und Fremdbesitzverbotes ermöglicht, wie die Erfahrungen im Einzelhandel und in anderen Ländern zeigen, die Bildung von finanzstarken Apo-

\footnotetext{
${ }^{101} \mathrm{Vgl}$. Sachverständigenrat zur Begutachtung der Entwicklung im Gesundheitswesen (2005), Ziffer 61.

${ }^{102}$ Das Vertragsverletzungsverfahren ist in Artikel 226 EGV geregelt, demzufolge Mitgliedstaaten und Europăische Kommission Verstöße eines Mitgliedstaates gegen europåisches Recht geltend machen können.

${ }^{103} \mathrm{Vgl}$. Europäische Kommission (2006d).

${ }^{104}$ Vgl. Blöß (2006), S. A2146.
} 
thekenketten und damit auch die Transformation des deutschen Apothekenmarktes von einer atomistischen in eine oligopolistische Struktur. Für eine solche Lösung spricht, dass die Bildung von Angebotsketten beispielsweise im Lebensmitteleinzelhandel und bei Drogerien zu Größenvorteilen und zu einer erheblichen Intensivierung des Wettbewerbs führten, die in Form von Preissenkungen letztlich den Konsumenten zugute kam. ${ }^{105}$ Im Vergleich mit der heutigen atomistischen Marktstruktur mit ihren über 20.000 Abgabestellen existiert, wie auch die Entwicklung in Norwegen verdeutlicht ${ }^{106}$, zumindest im weiten Oligopol ${ }^{107}$ eine erheblich höhere Wettbewerbsintensität.

Eine weitgehende Liberalisierung des Apothekenbesitzes und die dadurch induzierte Bildung von Apothekenketten dürften auch kaum zu einer Gefährdung der flächendeckenden Versorgung in Deutschland führen. Es ist nicht einsichtig, warum eine zunehmende Kettenbildung zu einer Schließung von zahlreichen Apotheken in bestimmten Gebieten (z.B. ländlichen Regionen) führen sollte. Eine Apotheke, die unter den derzeitigen Bedingungen wirtschaftlich rentabel ist, wird dies auch nach der Liberalisierung des Apothekenbesitzes sein, da aufgrund der AMPreisV ein Preiswettbewerb bei verschreibungspflichtigen Arzneimitteln nicht möglich ist. Es ist vielmehr davon auszugehen, dass sich die Rentabilität einer Apotheke durch die Zugehörigkeit zu einer Kette, z.B. aufgrund günstigerer Einkaufskonditionen, verbessern wird. Aus ökonomischer Sicht ist darum zu erwarten, dass sich die Zahl und die Verteilung der Apotheken nach einer Liberalisierung des Apothekenbesitzes nicht signifikant verändern werden. Eine weitere Verbesserung der Versorgung kann sich zudem aufgrund der zu erwartenden Zunahme von Versandapotheken ergeben.

Bedenken gegen eine sofortige Freigabe von Mehr- und Fremdbesitz bestehen darum weniger aufgrund der dann zu erwartenden horizontalen Konzentrationsprozesse auf dem Apothekenmarkt. Die wettbewerbspolitisch weit größere Gefahr droht im Hinblick auf vertikale Konzentrationsprozesse, denn der Apotheken-Großhandel weist eine stark oligopolistische Struktur auf. Die zwei bzw. vier größten Unternehmen vereinen ca. 50 bzw. 75 Prozent des gesamten Umsatzes auf sich. Da es sich hier um Durchschnittswerte handelt, liegt der regionale Konzentrationsgrad teilweise wesentlich höher. Zumindest zwei dieser Großhandelsfirmen besitzen bereits zahlreiche Apotheken in anderen europäischen Ländern und verfügen auch über die Finanzkraft, weite Teile des deutschen Apothekenmarktes mit dem Ziel einer vertikalen Verschachtelung aufzukaufen. ${ }^{108}$ Den aufkaufenden Firmen käme dabei zugute, dass ein beachtli-

\footnotetext{
${ }^{105}$ Vgl. SVRKAiG(1995), Ziffer 416.

${ }^{106}$ In Norwegen wurden sowohl das Fremd- als auch das Mehrbesitzverbot von Apotheken im März 2001 aufgehoben. Daraufhin kam es rasch zur Ausbreitung von Apothekenketten, die bereits im September 2001 zwei Drittel des Apothekenmarktes unter sich aufteilten. Nachdem die Umsätze des Pharmaeinzelhandels in den Jahren 1995 bis 2001 jeweils um ca. 5 Prozent pro Jahr gestiegen waren, betrug dieses Wachstum im Jahre 2002 nur noch 1,4 Prozent (geschätzt). Es ist zu vermuten, dass hierin die erhöhte Wettbewerbsintensităt durch die Liberalisierung der Arzneimitteldistribution zum Ausdruck kommt, zumal in diesem Zeitraum keine Maßnahmen ergriffen wurden, die einen Rückgang des Arzneimittelverbrauchs in Norwegen erklären würden. Vgl. Glaeske et al. (2003), S. $118 \mathrm{ff}$.

${ }^{107}$ Im engen Oligopol besteht die Gefahr von kartellartigem Verhalten, wie z.B. Preisabsprachen.

${ }^{108}$ Vgl. SVRKAiG(2000/2001), Addendum, Ziffer 68.
} 
cher Teil der Apotheken keinen hohen Geschäftswert besitzt - 42 Prozent der Apotheken erreichen lediglich einen Umsatz von bis zu 1 Mio. $€$ pro Jahr ${ }^{109}$. Da das marktführende Großhandelsunternehmen Phoenix, das mit seinem Umsatz 28 Prozent des Großhandelsmarktes einnimmt, dem gleichen Eigentümer gehört wie der größte Hersteller von Generika (Ratiopharm) wäre sogar eine vertikale Verschachtelung über drei Ebenen möglich.

Bei einer starken vertikalen Konzentration im Bereich der Arzneimitteldistribution, an der sich auch finanzkräftige Pharmahersteller beteiligen könnten, besteht die Gefahr, dass der Großhandel starken Einfluss auf die Sortimentpolitik der Apotheken nimmt oder sich durch eine enge oligopolistische bzw. eine monopolistische Marktstruktur in einzelnen Regionen im Bereich der nicht verschreibungspflichtigen Medikamente Möglichkeiten zu einer überhöhten Preissetzung bieten. Ein zentrales Problem einer Reform der Arzneimitteldistribution besteht somit darin, rechtliche Regelungen zu finden, die negativen Effekte einer vertikalen Verflechtung auf die Versicherten auszuschließen. Dies könnte beispielsweise geschehen, indem die Apotheken weiterhin zur Vorhaltung eines Vollsortiments verpflichtet bleiben oder aber bei einer weitergehenden Liberalisierung des Apothekenmarkts und einer Aufhebung des Kontrahierungszwangs die Krankenkassen als Vertragspartner der Apotheken Anforderungen an die Sortimentpolitik stellen können. ${ }^{110}$

Die ökonomischen Effekte, die sich aus einer Freigabe von Mehr- und Fremdbesitz ergeben können, hängen entscheidend von der Gestaltung anderer Rahmenbedingungen wie der Preisbildung und dem Kontrahierungszwang ab. Darum ist eine Abschätzung der Auswirkungen nur näherungsweise möglich. Glaeske et al. (2003) errechnen als grobe Näherung, dass bei einem Absinken der deutschen Handelsspannen auf das Mittel der derzeitigen Spanne in Deutschland und den Spannen in den USA, Großbritannien und Norwegen - also von Ländern mit Apothekenketten - im Bereich der GKV Einsparungen von über 1 Mrd. $€$ realisiert werden könnten. Ausgeschöpft werden könnte dieses Effizienzpotential beispielsweise durch die Einführung eines Preiswettbewerbs, indem von bundeseinheitlichen Apothekenpreisen zu Höchstpreisen für Arzneimittel übergegangen wird und kassenindividuelle Preis- und Rabattvereinbarungen ermöglicht werden. ${ }^{11}$

Ein Einfluss der Europäischen Integration war auch bei der Zulassung des Versandhandels mit Arzneimitteln zu erkennen. Zwar erfolgte die Verabschiedung des GMG, das zu entsprechenden Änderungen der deutschen Regelungen führte, bereits vor dem relevanten EuGH-Urteil in der Rechtssache "Deutscher Apothekerverband“ vom 11.12.2003. Dennoch dürften diese Änderungen von den Erwartungen der Gesundheitspolitik bezüglich des Urteils beeinflusst worden sein. Der Bundesverband Deutscher Apothekerverbände (ABDA) hatte gegen die Internetapotheke DocMorris und deren damaligen Betreiber Jacques Waterval geklagt, da sie trotz des in Deutschland geltenden Versandhandelsverbots Arzneimittel an deutsche Kunden und Patienten lie-

\footnotetext{
${ }^{109}$ Vgl. Bundesvereinigung Deutscher Apothekerverbände (ABDA) (2002), S. 42.

${ }^{110} \mathrm{Vgl}$. Glaeske et al. (2003), S. 134.

III Vgl. Glaeske et al. (2003), S. 135.
} 
ferten. Das zuständige Frankfurter Landgericht verwies den Fall zur Vorabentscheidung an den EuGH, weil es sich um einen grenzüberschreitenden Zusammenhang handelte, der die Grundfreiheiten - insbesondere die Warenverkehrsfreiheit - berührte. Die Generalstaatsanwältin forderte in ihrem Schlussantrag vor dem EuGH eine Freigabe des grenzliberschreitenden Versandhandels mit im jeweiligen Land zugelassenen Arzneimitteln. ${ }^{112}$ Der Gerichtshof folgte diesem Antrag lediglich teilweise. Während er eine Beschränkung des grenzüberschreitenden Versandhandels mit nicht verschreibungspflichtigen Medikamenten als europarechtswidrig ansah, entschied er in Bezug auf verschreibungspflichtige Arzneimittel, dass ein Versandhandelsverbot zum Schutz der Gesundheit und des Lebens der Menschen trotz des Konflikts mit der Warenverkehrsfreiheit zu rechtfertigen sei. ${ }^{113}$ Dass die Liberalisierung der deutschen Regelungen mit dem GMG über die Forderungen des Gerichtshofes hinausging, kann darauf zurückzuführen sein, dass die politischen Entscheidungsträger ein liberaleres Urteil erwartete hatten und dieses in der Gesetzesänderung vorwegnahmen. ${ }^{114}$ Es ist jedoch auch denkbar, dass mit der Liberalisierung des Versandhandels für alle Arzneimittel die Erwartung signifikanter Kosteneinsparungen für die gesetzlichen Krankenkassen gerade auch durch einen verstärken Bezug von Arzneimitteln im Ausland - verbunden wurde.

Der Anreiz zum verstärkten Bezug von Arzneimitteln aus anderen EU-Mitgliedstaaten ergibt sich bei frei verkäuflichen Medikamenten aus den in Abschnitt 3.3.1.4.4 skizzierten Preisdifferenzen innerhalb der EU. Im Fall rezeptpflichtiger Arzneimittel sind eventuelle Unterschiede in den Zuzahlungsbedingungen entscheidend. ${ }^{115}$ Zur Abschätzung, inwieweit die erwähnten Preisunterschiede zu Kosteneinsparungen führen können, ist interessant, in welchen europäischen Ländern die Endverbraucherpreise jene in Deutschland unterschreiten und wie groß diese Differenzen ausfallen. Verglichen werden müssen hierbei die Apothekenabgabepreise, da sie die Belastung der Patienten bzw. der Kostenträger widerspiegeln. ${ }^{116}$ Auf Basis dieser Preise und ausgehend von der Mengenstruktur des deutschen Arzneimittelmarktes errechnete die Beratungsgesellschaft für angewandte Systemforschung (BASYS) eine Indexzahl, die Schlüsse über die relevanten Preisdifferenzen zulässt (vgl. Abbildung 8). ${ }^{117}$ Die Studie kommt auf Basis des deutschen Warenkorbs - zu dem Ergebnis, dass die Arzneimittelpreise in Deutschland niedriger sind als in vielen anderen europäischen Ländern. Nur Griechenland, Spanien, Portugal und Frankreich besitzen nach dieser Berechnung im Durchschnitt geringere Preise.

\footnotetext{
112 Vgl. Europäischer Gerichtshof (2003a), Rn. 261.

${ }^{113}$ Vgl. Europäischer Gerichtshof (2003d), Rn. 117-119.

114 So Jasper/Tostmann (2004), S. 30.

${ }^{115}$ Für eine ausführliche Darstellung der in Deutschland geltenden Zuzahlungsbedingungen sowie zu den bei ausländischen Versandapotheken geltenden Bedingungen siehe den folgenden Abschnitt.

${ }^{116}$ Nicht beruicksichtigt wurden dabei Rabatte, die in den Mitgliedstaaten auf den unterschiedlichen Ebenen zum Tragen kommen, wie z.B. der 5-prozentige Kassenrabatt für die GKV in Deutschland. Vgl. Schneider (1999), S. 44ff.

${ }^{117}$ Dies ist die derzeit aktuellste Studie zum Vergleich der Arzneimittelabgabepreise. Die neuen Mitgliedstaaten wurden damals nicht in die Untersuchung einbezogen, da sie erst 2004 der Europåischen Union beitraten.
} 
Abbildung 8: Laspeyres-Preisindex des Apothekenabgabepreises (Vergleichsmaßstab: Devisenkurs) $)^{118}$

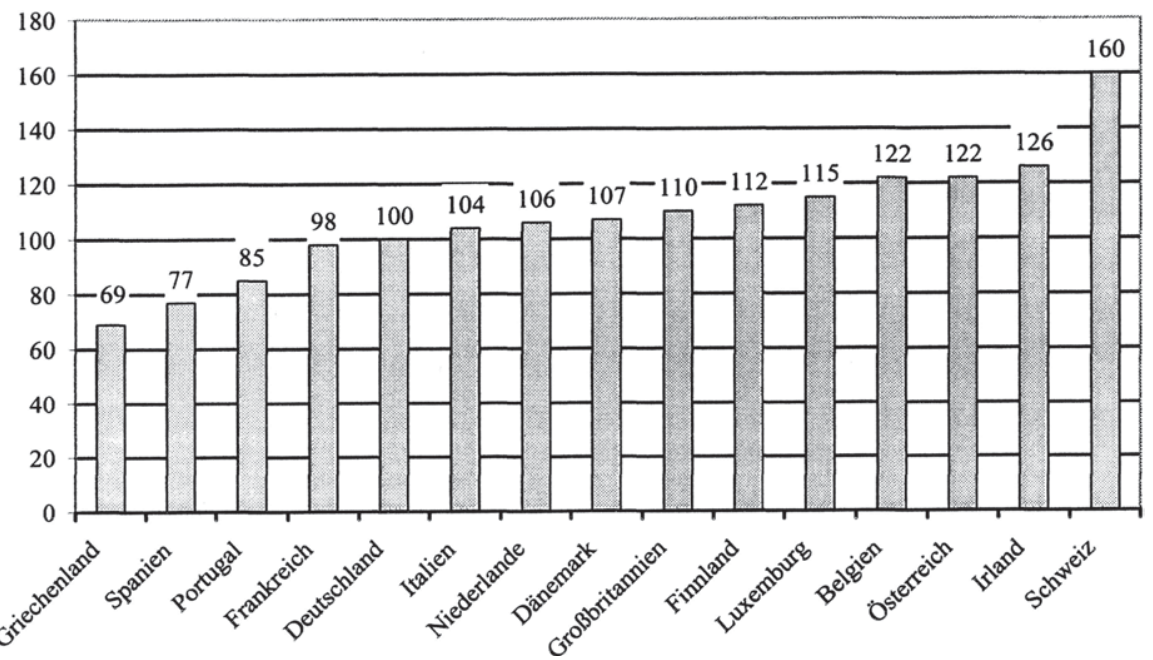

Quelle: Schneider (1999)

Die Indexzahl kann zu einer groben Abschätzung der Ersparnis verwendet werden, die die gesetzlichen Krankenkassen durch einen verstärkten Einkauf von Medikamenten im Ausland realisieren könnten. Die Arzneimittelausgaben der GKV betrugen im Jahre 2005 ca. 25,4 Mrd. $\epsilon^{119}$. Wenn alle zu Lasten der GKV nachgefragten Arzneimittel in einem Land gekauft worden wären, dessen Preise durchschnittlich um 1 Prozent unter den deutschen lagen, hätte sich für die GKV eine Einsparung von 254 Mio. $€$ ergeben. Mit jedem Prozentpunkt, um den die Preise im Ausland die deutschen unterschreiten, steigt die potentielle Ersparnis um diesen Betrag. Tabelle 1 zeigt unter Zugrundelegung der von BASYS berechneten Indexzahlen die Untergrenze des Einsparpotentials unter der Annahme, dass die im Jahre 1999 ermittelten Preisdifferenzen auch im Jahre 2005 unverändert galten.

Das hierbei errechnete maximale Einsparpotential bei einem Einkauf aller erstatteten Arzneimittel in Griechenland von 7,87 Mrd. $€$ hätte im Jahre 2005 zu eine Senkung der gesamten Leistungsausgaben um 5,78 Prozent geführt. Da im Jahre 2005 ein Beitragssatzpunkt Einnahmen in Höhe von 9,6 Mrd. $€$ entsprach, ${ }^{120}$ hătte damit eine spürbare Senkung des durchschnittlichen Beitragssatzes um 0,8 Punkte erreicht werden

\footnotetext{
${ }^{118}$ Bei der Berechnung des Indexes wurde der deutsche Warenkorb zugrunde gelegt, was die - im Vergleich zu anderen Studien - relativ geringe Indexzahl für Deutschland erklärt. Vgl. auch Anmerkung 1 zu Tabelle 5.

${ }^{119} \mathrm{Vgl}$. Bundesministerium für Gesundheit (BMG) (2006a).

${ }^{120} \mathrm{Vgl}$. Bundesministerium für Gesundheit (BMG) (2006b), S. 2.
} 
können. Die in Abbildung 8 dargestellten Indexzahlen geben jedoch keine Auskunft darüber, inwieweit einzelne Arzneimittel(gruppen) in anderen europäischen Ländern günstiger sind. Bei einem gezielten Einkauf von Arzneimitteln in den Ländern, in denen sie am billigsten sind, könnten daher noch weitere Kosteneinsparungen erzielt werden.

Tabelle 13: Abschätzung der Einsparmöglichkeiten durch den Einkauf von Arzneimitteln im Ausland für das Jahr 2005

\begin{tabular}{|c|c|c|c|c|c|}
\hline & $\begin{array}{l}\text { In- } \\
\text { dex }^{1)}\end{array}$ & $\begin{array}{l}\text { Ausgaben der } \\
\text { GKV für Arz- } \\
\text { neimittel } 2005 \\
\text { in Mrd. € }\end{array}$ & $\begin{array}{l}\text { Einspar- } \\
\text { potential } \\
\text { in Mrd. } \epsilon\end{array}$ & $\begin{array}{l}\text { Einsparpotential } \\
\text { in Prozent der } \\
\text { GKV- } \\
\text { Arzneimittel- } \\
\text { ausgaben } 2005\end{array}$ & $\begin{array}{l}\text { Einspar- } \\
\text { potential in Pro- } \\
\text { zent der GKV- } \\
\text { Leistungs- } \\
\text { ausgaben } 2005^{2)}\end{array}$ \\
\hline Deutschland & 100 & 25,4 & 0,0 & - & - \\
\hline Frankreich & 98 & 24,9 & 0,5 & 2,0 & 0,38 \\
\hline Portugal & 85 & 21,6 & 3,8 & 15,0 & 2,82 \\
\hline Spanien & 77 & 19,6 & 5,8 & 23,0 & 4,33 \\
\hline Griechenland & 69 & 17,5 & 7,9 & 31,0 & 5,84 \\
\hline
\end{tabular}

1) Berechnet durch BASYS auf Grundlage der Apothekenverkaufspreise; Vergleichsmaßstab: Devisenkurse

2) GKV-Leistungsausgaben 2005 insgesamt: 134,9 Mrd. $€$ (vgl. Kassenärztliche Bundesvereinigung (KBV) (2006))

Quelle: Schneider (1999); Bundesministerium für Gesundheit (BMG) (2006); eigene Berechnungen

Eine Liberalisierung der Gesundheitsmärkte allein lässt bei gegebenem Ordnungsrahmen allerdings noch keinen verstärkten Einkauf von Arzneimitteln im Ausland erwarten, da die derzeit zulässigen Distributionswege es den Krankenkassen nicht erlauben, selbst Medikamente zu kaufen. Eine Möglichkeit, wie die Krankenkassen direkt von geringeren Arzneimittelpreisen im Ausland profitieren könnten, stellt der Übergang zu einem sog. Einkaufsmodell dar. Die Kassen beziehen dann die Arzneimittel direkt aus dem Ausland und geben sie an ihre Versicherten weiter. Bei der Abschätzung des hierdurch realisierbaren Einsparpotentials gilt es jedoch einen wichtigen Aspekt zu berücksichtigen: Ein Teil der Preisdifferenzen zwischen den europäischen Mitgliedstaaten geht auf die unterschiedliche Höhe des jeweiligen Umsatzsteuersatzes zurück (vgl. Tabelle 7 auf Seite 140). In der EU gilt für Endverbraucher das Ursprungslandprinzip, d.h. bei einem Einkauf von Medikamenten im Ausland wird fur sie der dort geltende Umsatzsteuersatz fällig, so dass sie die Differenzen im Bruttopreis voll nut- 
zen können. ${ }^{121}$ Einkäufe von Gewerbetreibenden im europäischen Ausland unterliegen dagegen dem Bestimmungslandprinzip, wodurch ihnen ein geringerer ausländischer Umsatzsteuersatz nicht zugute kommt. $\mathrm{Zu}$ prüfen ist also, ob eine Krankenkasse bei einem Einkauf im Ausland rechtlich als Endverbraucher oder als Gewerbetreibender einzustufen ist. Durch die teilweise erheblichen Differenzen in den Steuersätzen besitzt diese Entscheidung einen großen Einfluss auf die möglichen Einsparungen der Krankenkassen.

Da die Krankenkassen unter dem gegebenen Ordnungsrahmen jedoch Arzneimittel nicht selbst kaufen dürfen, ist das Ausmaß der zu erwartenden Einsparungen wesentlich abhängig von den Anreizen zum Import von Arzneimitteln für Privatpersonen. Wichtige Determinanten hierbei sind die Ausgestaltung der Zuzahlungsregelungen im Inland sowie die Höhe der Transaktionskosten für einen Einkauf im Ausland. Durch das Entstehen von ausländischen Internet-Versandapotheken, die auch deutschsprachige Webseiten bereitstellen, sanken letztgeannte Kosten in den vergangenen Jahren erheblich. Mit den entsprechenden Anreizen durch die in Deutschland geltenden Zuzahlungsregelungen sowie die in den letzten Jahren entstandenen Internetapotheken beschäftigt sich der nachfolgende Abschnitt.

\subsubsection{Zuzahlungen und Versandapotheken}

Für die Versicherten besteht seit einigen Jahren die Möglichkeit, Arzneimittel beispielsweise über das Internet bei Versandapotheken im Ausland zu Preisen zu bestellen, die zum Teil deutlich unter den deutschen Apothekenabgabepreisen liegen. Damit eine Lieferung nach Deutschland möglich ist, müssen die ausländischen Apotheken die nachfolgend aufgeführten Anforderungen kumulativ erfüllen: ${ }^{122}$

- das Arzneimittel muss im Geltungsbereich des Gesetzes über den Verkehr mit Arzneimitteln (Arzneimittelgesetz, AMG) zugelassen oder registriert oder hiervon freigestellt sein,

- das Arzneimittel muss zur Anwendung am oder im menschlichen Körper bestimmt sein,

- der Versand erfolgt aus einer Apotheke eines Mitgliedstaates der EU oder des EWR,

- die versendende Apotheke ist zum Versandhandel befugt, entweder nach dem deutschen Apothekengesetz oder nach dem jeweiligen nationalen „Heimatrecht“ der Apotheke,

\footnotetext{
${ }^{121}$ Vgl. Reding/Müller (1999), S. 442ff. Bei der Bestellung von Arzneimitteln durch Versicherte im Ausland dürfen die Umsätze kleiner. Versandhäuser bzw. geringvolumige Versendungslieferungen ebenfalls nach dem Ursprungslandprinzip abgerechnet werden, vgl. Reding/Müller (1999), S. 445. Für die Versicherten bedeutet dies, dass bei der Bestellung von Arzneimitteln im europäischen Ausland die ausländische Umsatzsteuer anfallt, solange der Umsatz des Versenders bestimmte Schwellen nicht überschreitet.

${ }^{122} \mathrm{Vgl}$. Bundesministerium fulr Gesundheit und Soziale Sicherung (BMGS) (2005d).
} 
- der Versand des Medikamentes erfolgt entsprechend den deutschen Vorschriften zum Versandhandel oder zum elektronischen Handel.

Der Versand nach Deutschland ist demnach Apotheken nur dann gestattet, wenn sie u.a. nach jeweiligem nationalem oder deutschem Recht zum Versand von Arzneimitteln befugt sind und eine deutschsprachige Beratung anbieten. Der Versandhandel mit Arzneimitteln ist in der EU und im EWR bisher in den Niederlanden, der Schweiz und Großbritannien zugelassen. Es ist davon auszugehen, dass sich ein Versand in signifikanter Größenordnung nach Deutschland derzeit nur für Apotheken aus diesen Ländern lohnt, da sie bereits über die notwendigen Vertriebsstrukturen verfügen bzw. diese auch für den Versand in ihren Heimatländer nutzen können. Das Bundesministerium hat im Juni 2005 festgestellt, dass Apotheken aus den Niederlanden und Großbritannien generell Arzneimittel nach Deutschland versenden dürfen, da die dort geltenden Sicherheitsstandards mit den deutschen vergleichbar seien. ${ }^{123}$ Apotheken aus anderen europäischen Ländern können eine Versanderlaubnis entsprechend dem Apothekengesetz beantragen. Bisher existieren relativ wenige ausländische Apotheken, die im deutschen Markt tätig sind. Neben vier holländischen Internetapotheken (DocMorris, Europa Apotheek Venlo, Bonimed, Pharmakontor) existiert ein englischer Anbieter (Getpharma) mit deutschsprachiger Homepage, der allerdings nach eigenen Angaben nicht nach Deutschland versendet, sowie eine tschechische Versandapotheke (VfG), die sich bisher auf den Verkauf von lediglich ca. 600 freiverkäuflichen Heil- und Arzneimitteln beschränkt.

Einen Anreiz zu Preisvergleichen bei Arzneimitteln besitzen die Patienten vor allem im Bereich der Selbstmedikation, weil sie hier den vollen Arzneimittelabgabepreis selbst zahlen müssen. Nach Angaben der ABDA erzielten die deutschen Apotheken im Jahre 2005 durch die im Rahmen der Selbstmedikation gekauften Präparate einen Umsatz von 5,4 Mrd. $€$, was einem Anteil von 15,4 Prozent am gesamten Apothekenumsatz entspricht. ${ }^{124}$ Hinzu kommt ein Umsatz von 0,4 Mrd. $€$, der mit freiverkäuflichen Arzneimitteln in Drogerien und anderen Einzelhandelsgeschäften erzielt wurde. Durch die Freigabe der Preise für nicht verschreibungspflichtige Arzneimittel durch das GMG im Jahre 2004 ist allerdings auch in Deutschland ein harter Preiswettbewerb entstanden, der nach Angaben des Gründers der holländischen Versandapotheke DocMorris dazu führt, dass deutsche Apotheken z.B. nicht verschreibungspflichtige Produkte z.T. zu Einstandspreisen anbieten. ${ }^{125}$ Es ist deshalb davon auszugehen, dass durch die höhere Wettbewerbsintensität die zuvor bestehenden Preisdifferenzen zu ausländischen Anbietern deutlich gesunken sind und das Einsparpotential sowie der damit verbundene Anreiz zum Einkauf im Ausland in diesem Bereich abgenommen haben.

Bei rezeptpflichtigen Arzneimitteln erfolgt in Deutschland weiterhin eine Preisregulierung, die zu einem bundesweit einheitlichen Apothekenabgabepreis führt. Erhalten Versicherte ein Medikament aufgrund einer ärztlichen Verordnung, übernimmt die

\footnotetext{
${ }^{123} \mathrm{Vgl}$. Bundesministerium für Gesundheit und Soziale Sicherung (BMGS) (2005b).

${ }^{124} \mathrm{Vgl}$. Bundesvereinigung Deutscher Apothekerverbände (ABDA) (2006), S. 46.

${ }^{125}$ Vgl. o.V. (2005b).
} 
Krankenkasse die Kosten bis auf eine Zuzahlung, die die Patienten tragen müssen. Die Versicherten werden daher nur dann verschreibungspflichtige Medikamente aus dem Ausland beziehen, wenn die Ausgestaltung der Zuzahlungsregelungen bzw. eventuelle Rabatte der ausländischen Anbieter dafür sorgen, dass für sie ein finanzieller Vorteil resultiert. In Deutschland beträgt die Zuzahlung 10 Prozent des Apothekenabgabepreises, mindestens aber $5 €$ und maximal $10 €{ }^{126}$ Für Arzneimittel, die weniger als $5 €$ kosten, zahlt der Versicherte lediglich den tatsächlichen Preis. Abbildung 9 zeigt die sich daraus ergebende Selbstbeteiligung in Abhängigkeit vom Apothekenabgabepreis.

Abbildung 9: Selbstbeteiligung der Patienten auf Arzneimittel in Deutschland

Selbstbeteiligung in Euro

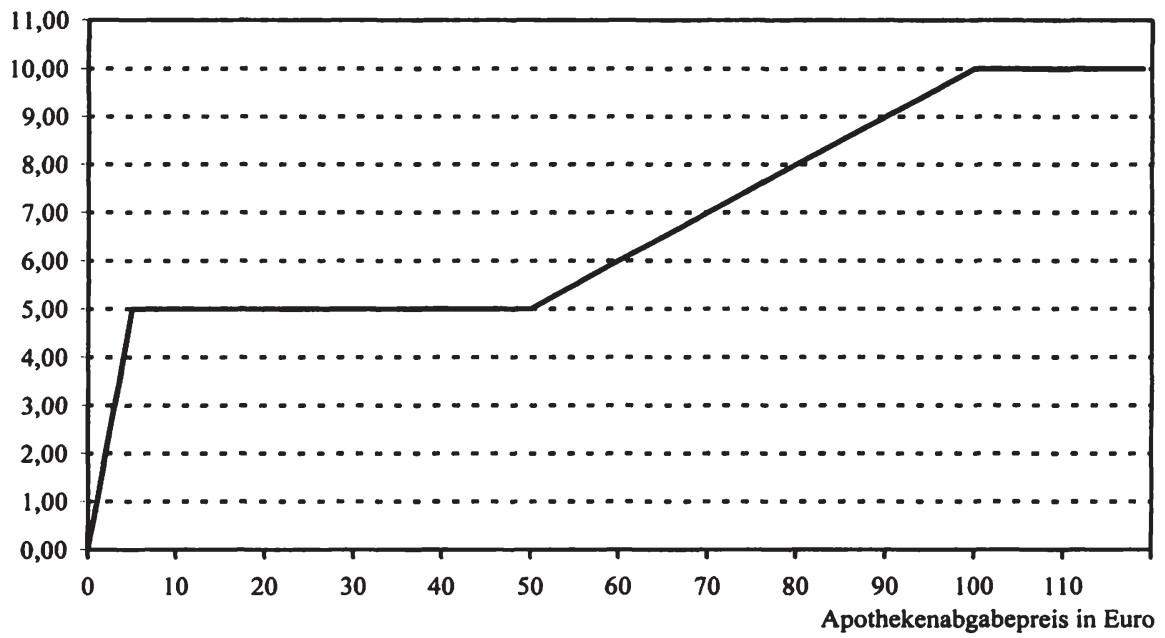

Quelle: § 61 SGB V; eigene Darstellung

Dieser Tarif besteht demnach aus vier Bereichen. Bis zu einem Apothekenabgabepreis von $5 €$ zahlt der Patient 100 Prozent des Preises. Bei einem Preis zwischen 5 und $50 €$ sowie ab $100 €$ wird ein fixer Betrag von 5 bzw. $10 €$ fällig; im Bereich von 50 bis $100 €$ ist der Tarif proportional ausgestaltet. Ein Patient besitzt generell nur bei einer prozentualen Selbstbeteiligung den Anreiz, nach preisgünstigen Angeboten zu suchen. Bei der derzeitigen Regelung trifft dies also lediglich für die Bereiche zwischen 0 und $5 €$ sowie zwischen 50 und $100 € \mathrm{zu}$. Rabatte auf die gesetzlichen Zuzahlungen sind deutschen Apotheken gemäß Heilmittelwerbegesetz verboten. ${ }^{127}$

Die ausländischen Anbieter haben gegenüber den deutschen Apotheken den Vorteil, dass sie dem deutschen Gesetz nicht unterliegen und daher - wenn es ihre nationalen

\footnotetext{
${ }^{126}$ Vgl. § 31 Abs. 3 SGB V i.V.m. § 61 SGB V.

${ }^{127} \mathrm{Vgl}$. $§ 7$ Abs. 1 Nr. 2 des Gesetzes über die Werbung auf dem Gebiete des Heilwesens (Heilmittelwerbegesetz, HWG).
} 
Regelungen erlauben - neben günstigeren Preise für rezeptpflichtige Arzneimittel auch andere Vergünstigungen anbieten können, die direkt den Patienten zugute kommen. So verlangt die hollåndische Versandapotheke DocMorris bei rezeptpflichtigen Medikamenten von den Versicherten lediglich die Hälfte des jeweiligen in Deutschland fälligen Zuzahlungsbetrags. ${ }^{128}$ Die Ersparnis liegt für die Versicherten also zwischen 2,50 und $5 €$. Zudem entfallen die Versandkosten, wenn mindestens zwei rezeptpflichtige Medikamente bestellt werden. Die Europa Apotheek in Venlo (Niederlande) bietet ihren Kunden für die Bestellung von rezeptpflichtigen Arzneimitteln einen Bonus in Höhe von drei Prozent des in Deutschland geltenden Arzneimittelabgabepreises. ${ }^{129} \mathrm{Bei}$ Preisen unter 83,33 $€$ beträgt dieser Bonus generell $2,50 €,{ }^{130}$ bei Preisen über $500 €$ einheitlich $15 €$. Der Bonus wird in der Regel sofort eingelöst und reduziert den von den Versicherten zu zahlenden Selbstbehalt, der damit unterhalb der in Deutschland nach § 61 SGB V anfallenden Zuzahlung liegt. Der Teil des Bonus, der die jeweils in Deutschland geltende Selbstbeteiligung übersteigt, ${ }^{131}$ wird ggf. auf den Preis von zeitgleich bestellten freiverkäuflichen Arzneimitteln angerechnet oder dem Patienten auf einem Kundenkonto gutgeschrieben und mit späteren Bestellungen verrechnet. Zudem fallen für rezeptpflichtige Arzneimittel keine Versandkosten an. Abbildung 10 zeigt die jeweilige Belastung der Patienten in Abhängigkeit vom deutschen Apothekenabgabepreis bei einer Bestellung bei DocMorris und der Europa Apotheek im Vergleich $\mathrm{zu}$ der in Deutschland anfallenden gesetzlichen Selbstbehalten. ${ }^{132}$

Die Abbildung zeigt, dass eine Bestellung von rezeptpflichtigen Medikamenten bei der Europa Apotheek Venlo für die Versicherten über den gesamten Preisbereich einen finanziellen Vorteil gegenüber dem Bezug in Deutschland mit sich bringt. Die Bestellung von Arzneimitteln bei der Internetapotheke DocMorris bietet lediglich bei Apothekenabgabepreisen bis $5 €$ keine Reduktion der Selbstbeteiligung gegenüber der Einlösung des Rezepts in einer deutschen Apotheke. Die Kunden müssen innerhalb dieses Preisbereichs bei der holländischen Apotheke ebenfalls den vollen Arzneimittelabgabepreis als Selbstbeteiligung tragen. Dagegen erhalten Kunden der Europa Apotheek Venlo auch bei Preisen unter $5 €$ bereits einen Bonus von 2,50€ pro Medikament und müssen lediglich den ggf. anfallenden Restbetrag selbst tragen. Im Bereich zwischen 5 und $50 €$ bieten die beiden Anbieter die gleiche Ersparnis von 2,50€ pro Arzneimittel. Bei einem Medikamentenpreis zwischen 50 und $166,66 €$ ist der Selbstbehalt fuir die

\footnotetext{
${ }^{128} \mathrm{Vgl}$. DocMorris (2006).

${ }^{129}$ Vgl. Europa Apotheek Venlo (2006).

${ }^{130}$ Bei Apothekenabgabepreisen von unter 2,50€ wäre damit der gewăhrte Bonus höher als der Preis des Arzneimittels, so dass die Apotheke beim Versand dieser Medikamente einen negativen Deckungsbeitrag erwirtschaften würde. Es ist jedoch davon auszugehen, dass nur sehr wenige verschreibungspflichtige Arzneimittel in Deutschland einen Apothekenabgabepreis aufweisen, der weniger als $2,50 €$ beträgt.

${ }^{131}$ Dies gilt zum einen - wie bereits erwähnt -, wenn der Apothekenabgabepreis geringer ist als 2,50 $€$, zum anderen, wenn der Apothekenabgabepreis höher ist als 333,33 $€$. Der von der Europa Apotheek gewăhrte Bonus übersteigt im zweiten Fall nämlich den maximalen Zuzahlungsbetrag in Deutschland von $10 €$ pro Arzneimittel.

${ }^{132}$ Es wird bei einer Bestellung bei DocMorris angenommen, dass mindestens zwei rezeptpflichtige Arzneimittel bestellt werden, so dass die Versandkosten entfallen.
} 
Patienten bei DocMorris am geringsten. Für Preise zwischen 166,66€ und 333,33€ verlangt dagegen die Europa Apotheek Venlo die geringste Selbstbeteiligung für rezeptpflichtige Arzneimittel, die bis zu einem Arzneimittelabgabepreis von $500 €$ mit der Höhe des Abgabepreises kontinuierlich sinkt. Da der von der Europa Apotheek Venlo gewährte Bonus ab einem Preis von 333,33€ höher ist als die in Deutschland fällige Zuzahlung, kann der Differenzbetrag mit zukünftig anfallenden Zuzahlungen oder freiverkäuflichen Medikamenten verrechnet werden. Diese Form der Rabattregelung bietet insbesondere bei hochpreisigen Arzneimitteln einen Anreiz, diesen holländischen Anbieter zu nutzen. Vorteilhaft für die Wettbewerbschancen dieser Apotheke ist auch die positive Bewertung durch die Stiftung Warentest vom 22. Februar 2005. In diesem Test beurteilte die Stiftung 20 Versandapotheken im In- und Ausland; neun von ihnen erhielten das Qualitätsurteil „gut" - darunter auch die Europa Apotheek Venlo. Die Versandapotheke DocMorris schloss den Test aufgrund fehlerhafter und unzureichender Beratung mit dem Urteil "mangelhaft" $a b$.

Abbildung 10: Vergleich der Selbstbeteiligung der Patienten für rezeptpflichtige Arzneimittel

Selbstbeteiligung in Euro

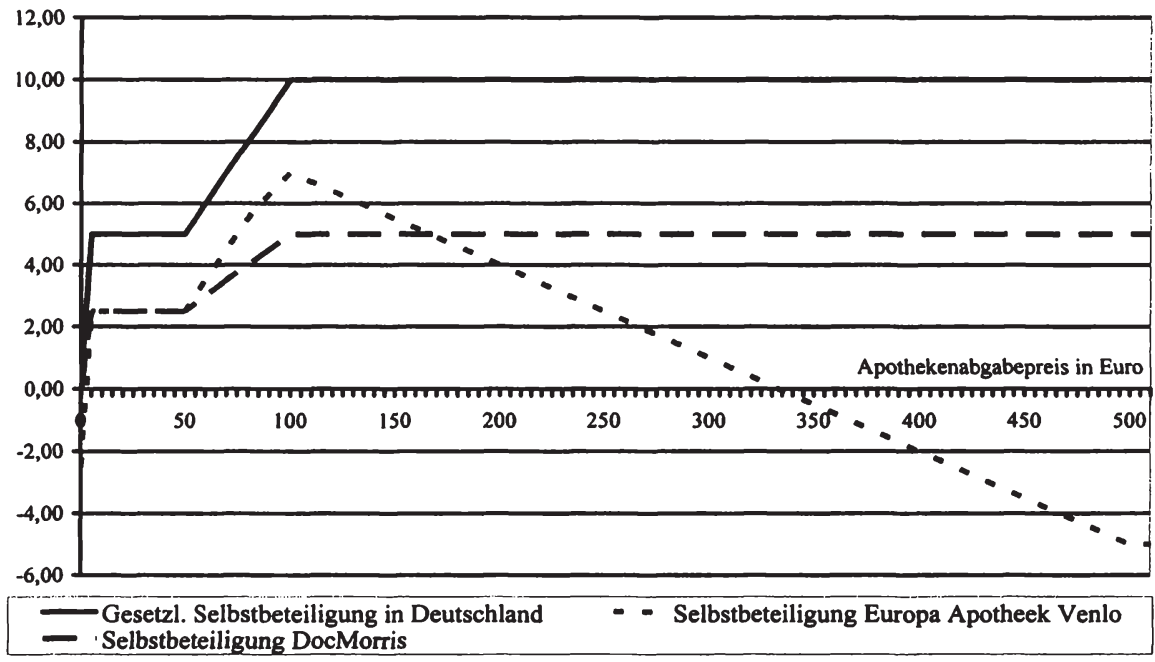

Quelle: § 61 SGB V; Homepages der Versandapotheken; eigene Darstellung.

Doch auch die deutsche Versandapotheke „Zur Rose“ aus Halle hat trotz des restriktiven gesetzlichen Rahmens eine Möglichkeit gefunden, den Versicherten einen kleinen finanziellen Anreiz zur Bestellung zu geben. Für jedes eingereichte Rezept mit mindestens zwei rezeptpflichtigen Medikamenten erhalten ihre Kunden einen Warengutschein über $5 €$, der beim nächsten Einkauf rezeptfreier Medikamente eingelöst werden kann. Dennoch sind die Möglichkeiten der ausländischen Anbieter, mit Rabattaktionen Kunden anzuwerben, deutlich größer. 
Der IKK Bundesverband veröffentlichte im Februar 2005 erste ausgewertete Detailergebnisse darüber, welche IKK-Versicherten den Arzneimittelversandhandel mit der Apotheke Sanicare am intensivsten nutzen. ${ }^{133}$ Der IKK Bundesverband hatte mit diese Apotheke zum 1.4.2004 einen Rahmenvertrag zum Versandhandel geschlossen. Die Studie zeigt, dass es sich bei einem Großteil der bestellten Medikamente um hochpreisige Arzneimittel handelt, die insbesondere zur Behandlung von Multipler Sklerose, Diabetes und HIV/Aids angewandt werden. Damit bestätigt sich die Erwartung, dass der Arzneimittelversandhandel vor allem von chronisch kranken Patienten genutzt wird, die eine Dauermedikation benötigen. Überraschend sind die Ergebnisse bezüglich der Altersstruktur der Versandhandelsnutzer. Demnach bestellte - gemessen an der Zahl der Einzelverordnungen - keine Altersgruppe mehr Medikamente bei der Versandapotheke als die Gruppe der 65- bis 69-Jährigen (13,9 Prozent der Einzelverordnungen). ${ }^{134}$ Dahinter folgte die Gruppe der 75 bis 79-Jährigen, auf die 11,2 Prozent aller bestellten Medikamente entfielen. ${ }^{135}$ Durch die überdurchschnittliche Morbidität in den höheren Altersklassen und dem damit verbundenen höheren Arzneimittelverbrauch kann aus diesen Zahlen nicht geschlossen werden, dass der Anteil der Internetbesteller in den genannten Altersklassen höher ist als in jüngeren Gruppen. Überraschend ist jedoch, dass die Anteile der von den Altersklassen ab 65 bei Sanicare bestellten Medikamente an allen dort bestellen Medikamenten - wiederum gemessen an der Zahl der Einzelverordnungen - jeweils größer sind als die entsprechenden Anteile an der Zahl aller zu Lasten der IKK bezogenen Arzneimittel. ${ }^{136}$ Dies widerspricht der oftmals geäußerten Vermutung, dass das Angebot von Internet- und Versandapotheken überwiegend für jüngere Menschen interessant ist, während ältere Menschen den neuen Vertriebswegen generell skeptisch gegenüberstehen. Die hohe Wiederbestellquote, die sich innerhalb der Untersuchung ergibt, spricht weiterhin für eine große Zufriedenheit der Versicherten mit dem in Anspruch genommenen Anbieter.

Auch eine Studie der Postbank zum Medikamentenhandel im Internet zeigt, dass unerwartet viele Patienten, die über 60 Jahre als sind, bereits Arzneimittel im Internet gekauft haben. ${ }^{137}$ Die Bestellungen bezogen sich insgesamt vorwiegend auf rezeptfreie Arzneimittel und Wellnessprodukte. Die holländische Internet-apotheke DocMorris erzielte dagegen im Jahre 2005 ca. 90 Prozent ihres Umsatzes mit rezeptpflichtigen Arzneimitteln, was vorwiegend daraus resultiert, dass die deutschen Versandapotheken bei den rezeptpflichtigen Medikamenten gegenüber ihren ausländischen Konkurrenten den Nachteil haben, keine Nachlässe auf die gesetzlich vorgeschriebenen Selbstbeteiligungen der Patienten gewähren zu können.

\footnotetext{
${ }^{133} \mathrm{Vgl}$. IKK Bundesverband (2005), S. $11 \mathrm{ff}$.

${ }^{134}$ Eigene Berechnung auf Basis von IKK Bundesverband (2005), Tabelle 8, S. 12.

${ }^{135}$ Auf die Altersgruppen der unter 60-Jăhrigen entfielen jeweils zwischen 0,9 und 7,3 Prozent aller Einzelverordnungen.

${ }^{136} \mathrm{Vgl}$. IKK Bundesverband (2005), S. 14.

${ }^{137} 18,5$ Prozent der Befragten im Alter von 60 Jahren oder älter gaben an, bereits Medikamente im Internet gekauft zu haben. In der Gesamtstichprobe betrug dieser Anteil lediglich 8,8 Prozent. Vgl. Bundesverband Deutscher Postdienstleister (BvDP) (2005).
} 
Auch für die Krankenkassen ist das Geschäft mit Versandapotheken interessant. Für sie bietet diese Vertriebsform eine Möglichkeit, die nach § 130 a SGB V aushandelbaren Herstellerrabatte an die Versicherten weiterzugeben. Zudem können sie mit den Versandapotheken selbst Nachlässe vereinbaren. ${ }^{138}$ Zahlreiche Kassen haben bereits Verträge mit in- und ausländischen Apotheken abgeschlossen. Die Versicherten erhalten dann beispielsweise versandkostenfreie Lieferungen, Gutscheine, Erstattungen der Zuzahlung beim Arztbesuch oder Rabatte auf rezeptfreie Arzneimittel. Die IKK beispielsweise kooperiert mit der deutschen Internetapotheke Sanicare, die den IKKVersicherten einen Rabatt von mindestens 10 Prozent auf den empfohlenen Verkaufspreis für alle freiverkäuflichen Medikamente gewährt sowie exklusive Sonderpreise für ausgewählte aktuelle Apothekenprodukte und verschreibungsfreie Arzneimittel anbietet.

Der Arzneimittelversandhandel nimmt jedoch insgesamt noch einen sehr geringen Anteil am Gesamtmarkt ein. Derzeit sind in Deutschland rund 1000 Apotheken als Versandapotheken zugelassen. Hinzu kommen die oben genannten ausländischen Anbieter, die die gesetzlichen Auflagen erfüllen und insbesondere rezeptpflichtige Arzneimittel nach Deutschland liefern. Nach Angaben des Bundesverbands deutscher Versandapotheken (BVDVA) betrug der Anteil des Versandhandels am Arzneimittelumsatz in Deutschland im Jahre 2004 insgesamt ca. 0,5 bis 1,5 Prozent. ${ }^{139}$ Bei Arzneimittelumsätzen von ca. 32,8 Mrd. $€^{140}$ im Jahre 2005 erwirtschaften diese Apotheken damit insgesamt ein Umsatzvolumen zwischen rund 164 und 492 Mio. $€$. Auf der Grundlage dieser Daten lässt sich der Anteil der ausländischen Internetapotheken grob abschätzen. Der Bundesverband der deutschen Versandapotheken (BVDVA) gibt an, dass 70 Prozent der Versandhandelsumsätze mit Medikamenten in Deutschland von holländischen Apotheken erzielt werden, was auf Basis der hier zugrunde gelegten Daten einem Umsatz zwischen rund 114 Mio. und 344 Mio. $€$ entspräche. Da anderen ausländischen Akteuren im deutschen Apothekenmarkt keine bedeutende Rolle zukommt, ist darum davon auszugehen, dass der Umsatz ausländischer Versandapotheken einen Anteil von maximal rund einem Prozent des Gesamtarzneimittelmarktes einnimmt. Damit dürfte der Einfluss der ausländischen Versandapotheken für die deutschen Apotheker quantitativ bisher kaum spürbar sein.

Die größte ausländische Versandapotheke auf dem deutschen Markt ist die niederländische Internetapotheke DocMorris, die mit einem Umsatz von 150 Mio. $€$ im Jahre 2005 auch der Marktführer in diesem Segment war. Die Preise für verschreibungspflichtige Arzneimittel liegen bei DocMorris nach eigenen Angaben durchschnittlich 15 Prozent unter den deutschen Apothekenabgabepreisen. Bei dieser Preisdifferenz hätten - basierend auf den Daten des Jahres 2005 - über diese Internetapotheke Umsätze in Höhe von 1,44 Mrd. Euro zu Lasten der GKV abgerechnet werden müssen,

\footnotetext{
${ }^{138}$ Vgl. Sachverständigenrat zur Begutachtung der Entwicklung im Gesundheitswesen (2005), S. 306.

$139 \mathrm{Vgl}$. Lahm (2005). Bis Ende 2009 rechnet der Verband mit einem Anstieg auf acht Prozent des Arzneimittelmarktes.

${ }^{140}$ Darin enthalten sind die Arzneimittelumsătze der deutschen Apotheken sowie der Umsatz mit freiverkäuflichen Arzneimitteln außerhalb von Apotheken. Eigene Berechnungen auf Basis der Angaben der ABDA. Vgl. Bundesvereinigung Deutscher Apothekerverbănde (ABDA) (2006) , S. 46.
} 
um eine einprozentige Senkung der GKV-Arzneimittelausgaben ${ }^{141}$ zu erreichen. Der Umsatz der gesetzlichen Krankenkassen mit deutschen und ausländischen Versandhändlern betrug im Jahre 2004 laut Angaben des Bundesgesundheitsministeriums insgesamt allerdings lediglich rund 40 Mio. $€$, was einem Anteil von ca. 0,17 Prozent an den gesamten Arzneimittelausgaben der GKV entsprach. ${ }^{142}$

Folglich ist davon auszugehen, dass unter den derzeitigen Bedingungen auch langfristig keine gravierenden Einsparungen bei den Arzneimittelausgaben durch den Kauf im europäischen Ausland zu erwarten sind. Auch in diesem Bereich ist die Flexibilität der Versicherten und Patienten zu gering, um bedeutende Auswirkungen zu entfalten. Etwas anders könnte sich die Situation darstellen, wenn die gesetzlichen Krankenkassen im Rahmen der oben beschriebenen Einkaufsmodelle die Möglichkeit erhalten würden, Arzneimittel für ihre Patienten selbst einzukaufen. Aus heutiger Sicht bleibt dennoch festzuhalten, dass insbesondere die Apotheke DocMorris einen wesentlichen Beitrag zur Liberalisierung der deutschen Arzneimitteldistribution leistete. Ihr Eintritt in den deutschen Markt löste eine Diskussion über das damals bestehende Versandhandelsverbot aus, die letztlich zur Zulassung dieses Distributionsweges führte. Aus ökonomischer Sicht wäre es wünschenswert, dass die Patienten diese Möglichkeit verstärkt zu einem Einkauf bzw. einer Rezepteinlösung im Ausland nutzen, um weiterhin Bewegung in den deutschen Apothekenmarkt zu bringen. Denkbar wäre, dass die ausländische Konkurrenz letztlich dazu führt, dass eine weitere Preisfreigabe im Arzneimittelbereich politisch durchsetzbar wird, da auch die inländischen Apotheker ein Interesse daran gewinnen, durch einen stärkeren Preiswettbewerb im Segment der rezeptpflichtigen Arzneimittel Kunden zu gewinnen bzw. deren Abwanderung zu (ausländischen) Konkurrenten zu verhindern.

Eine weitere Regelung des deutschen Apothekenrechts steht ebenfalls mit europäischem Recht in Konflikt und wird langfristig daher keinen Bestand haben können. § 2 Satz 2 des Gesetzes über das Apothekenwesen schreibt vor, dass Bürger anderer Mitgliedstaaten selbst keine Apotheken eröffnen dürfen, sondern lediglich eine Apotheke übernehmen können, die bereits seit mindestens drei Jahren existiert. Dies stellt eindeutig eine Diskriminierung der ausländischen Apotheker gegenüber ihren inländischen Kollegen dar, die gegen die europäische Niederlassungsfreiheit verstößt. Das österreichische Apothekenrecht sieht eine analoge Regelung vor, die dazu fuihrte, dass die Europäische Kommission im Juni 2006 gegen Österreich ein Vertragsverletzungsverfahren einleitete, das als ersten Schritt eine Stellungnahme Österreichs zu diesen Vorwürfen vorsieht. ${ }^{143}$ Inwieweit sich aus der Aufgabe der entsprechenden Regelungen in Deutschland Auswirkungen auf die Apothekenlandschaft ergäben, hängt davon ab, wie attraktiv die Eröffnung einer Apotheke für ausländische Apotheker ist. Aufgrund der recht hohen Apothekendichte und der eher verhaltenen wirtschaftlichen

\footnotetext{
${ }^{141}$ Die GKV-Arzneimittelausgaben betrugen 2005 rund 25,39 Mrd. $€$.

142 Vgl. Apothekerkammer Westfalen-Lippe (2004).

${ }^{143} \mathrm{Vgl}$. Europäische Kommission (2006d).
} 
Entwicklung des Apothekenmarktes in den vergangenen Jahren ${ }^{144}$ ist jedoch davon auszugehen, dass sich daraus keine wesentlichen Veränderungen ergeben werden.

Insgesamt ist festzustellen, dass während der letzte Jahren Bewegung in den deutschen Apothekenmarkt gekommen ist. Durch die Freigabe der Preise für nicht verschreibungspflichtige Arzneimittel konnte sich eine Konkurrenzsituation entwickeln, die zu einem Absinken des Preisniveaus dieser Medikamente gefuhrt hat. Zudem ist durch die Zulassung des internationalen Versandhandels grenzüberschreitend ein Wettbewerb auf Basis der Arzneimittelpreise und der Patientenzuzahlungen möglich geworden, der insbesondere Personen zugute kommt, die eine Dauermedikation benötigen und damit einen planbaren Medikamentenbedarf aufweisen. Trotz dieser Entwicklungen gelten im Apothekenmarkt weiterhin zahlreiche Regulierungen, die dazu führen, dass die Apotheker vor einem intensiveren Wettbewerb geschützt sind. Im Hinblick auf die zu realisierenden Effizienzgewinne ist daher eine weitere Liberalisierung des Marktes durch die Aufgabe des Fremdbesitzverbots und eine weitere Lockerung der immer noch bezüglich des Mehrbesitzes bestehenden Beschränkungen wünschenswert. Wie die obigen Betrachtungen zeigen, könnten die europäischen Grundfreiheiten in näherer Zukunft diesbezügliche Überlegungen anstoßen.

${ }^{144} \mathrm{Vgl}$. Bundesvereinigung Deutscher Apothekerverbände (ABDA) (2005), S. 40ff. 


\section{Gesundheitssysteme im Wettbewerb}

Durch die zunehmende ökonomische Globalisierung und die schrittweise Liberalisierung der europäischen Märkte kommt es nicht nur zu einem zunehmenden Wettbewerbsdruck auf die Leistungserbringer im Gesundheitswesen. Auch die Konkurrenz der verschiedenen (Gesundheits-)Systeme verstärkt sich durch die größere Mobilität der Produktionsfaktoren und dabei insbesondere des Kapitals. Hierbei ergeben sich im Wettbewerb um mobile Unternehmen tendenziell Nachteile für die beitragsfinanzierten Gesundheitssysteme. Die Beiträge zur sozialen Krankenversicherung werden in den meisten europäischen Ländern von Arbeitnehmern und Arbeitgebern gemeinsam getragen und stellen daher für die Unternehmen einen Teil der Arbeitskosten dar. Aufgrund der Wachstumsschwäche der Finanzierungsbasis ${ }^{145}$ sind in Deutschland c.p. Beitragssatzsteigerungen notwendig, um die Ausgaben zu decken, was die Arbeitskosten zusätzlich belastet. Dieser Zusammenhang besteht in steuerfinanzierten Systemen in geringerem Maße, da höhere Gesundheitsausgaben von der Allgemeinheit über höhere Steuern finanziert werden und nicht direkt auf den Arbeitskosten lasten. Daher liegen die Lohnzusatzkosten in beitragsfinanzierten Systemen tendenziell höher als in überwiegend steuerfinanzierten, was die Produzenten von arbeitsintensiven Produkten im internationalen Wettbewerb benachteiligt. Aus diesem Grund ist die Abkopplung der Arbeitskosten von zukünftigen Beitragssatzsteigerungen ein Ziel vieler der in Deutschland derzeit diskutierten Reformvorschläge für die Finanzierung der Krankenversicherung. Ein erster Schritt in diese Richtung wurde im Sommer 2005 mit dem Zusatzbeitrag in Höhe von 0,9 Prozent unternommen, der von den Versicherten der Krankenkassen alleine zu tragen ist, während gleichzeitig alle Krankenkassen ihren bisherigen Beitragssatz um 0,9 Prozent senkten, wovon Kassenmitglieder und deren Arbeitgeber jeweils hälftig profitieren. Dadurch ergibt sich letztendlich eine Entlastung der Arbeitgeber in Höhe von 0,45 Prozent. In grenznahen Gebieten besteht zudem ein Wettbewerb um qualifizierte Arbeitskräfte, die als sog. Grenzgänger das Land ihrer Versicherungszugehörigkeit wählen können und damit die Höhe ihrer Steuern und Abgaben beeinflussen können, indem sie das Land mit der für sie geringsten Steuerund Abgabenlast auswählen.

Die Theorie besagt, dass sich aus diesem „Wettbewerb der Systeme“ Veränderungen der nationalen (Gesundheits-)Systeme ergeben können. Nach Hayek (1969) dient der Wettbewerb als Entdeckungsverfahren, indem er Tatsachen offen legt, „die ohne sein Bestehen entweder unbekannt bleiben oder doch zumindest nicht genutzt werden würden. ${ }^{\text {(146 }}$ Seine Ergebnisse sind unvorhersagbar und können auf anderen Wegen (z.B. durch zentrale Planung) nicht gewonnen werden, da den Akteuren das Wissen fehlt, um ihre Entscheidungen ohne die Abstimmung über den Markt derart zu koordinieren, dass die bestmögliche Allokation zustande kommt. Diese dynamische Funktion kommt dem Wettbewerb entsprechend der evolutorischen Theorie des „Wettbewerbs der Sys-

\footnotetext{
${ }^{145}$ Diese Wachstumsschwäche der Finanzierungsbasis bedeutet, dass die beitragspflichtigen Einnahmen zur Krankenversicherung schwächer wachsen als das Bruttoinlandsprodukt und die Gesundheitsausgaben.

${ }^{146}$ Hayek (1969), S. 249.
} 
teme" nicht allein auf ökonomischen Märkten zu. Vielmehr bietet die Konkurrenz unterschiedlicher institutioneller Arrangements die Möglichkeit, die Vor- und Nachteile der Systeme zu erkennen und kreative Problemlösungsansätze zu generieren, weil sie den Mitgliedern einer Gesellschaft ermöglicht, ihre Präferenzen nicht nur über die Beteiligung an politischen Wahlen, sondern auch durch die Abwanderung in ein anderes "Institutionenset" $\mathrm{zu}$ bekunden. ${ }^{147}$ Dies ist von großer Bedeutung für die Durchsetzung der Präferenzen im politischen Prozess, die sich allein mittels politischer Wahlen schwierig gestaltet, weil sich der Wettbewerb um Wählerstimmen durch eine zeitliche, personelle und sachliche Undifferenziertheit auszeichnet: ${ }^{148}$

- Wahlen finden nur in bestimmten zeitlichen Abständen statt, wodurch die Politiker in der Zwischenzeit einen großen diskretionären Handlungsspielraum besitzen (zeitliche Undifferenziertheit).

- Die von den gewählten Politikern angebotenen Produkte sind teilweise öffentliche Güter, weshalb sich der Nutzen einer Kontrolle des Politikers auf viele Individuen verteilt. $\mathrm{Da}$ aber die Kosten individuell getragen werden müssen, besteht ein Anreiz zum Trittbrettfahrerverhalten. Der Anreiz des einzelnen Wählers zur Kontrolle des Politikers ist also gering (personelle Undifferenziertheit).

- Zudem kann der Wähler meist nur über Parteiprogramme und nicht über einzelne Entscheidungen abstimmen (sachliche Undifferenziertheit).

Diese Undifferenziertheit der Wahlen und die „rationale Unwissenheit“ der Wähler aufgrund der hohen Kosten der Informationsbeschaffung und dem relativ geringen Einfluss des einzelnen Wählers auf den Wahlausgang führen dazu, dass der politische Wettbewerb die Kontroll- und Anreizfunktion für die Wähler wesentlich schlechter erfüllt als der marktliche Wettbewerb für die Konsumenten. ${ }^{149}$

Besteht jedoch eine Konkurrenz verschiedener institutioneller Arrangements, können die Bürger ihre Präferenzen bezüglich der Ausgestaltung des institutionellen Rahmens offenbaren und durchsetzen, indem sie in das ihren Wünschen (besser) entsprechende System abwandern (exit). Der Wettbewerb unterschiedlicher Systeme kann dabei nicht allein durch die Abwanderung von Produktionsfaktoren, sondern auch durch grenzüberschreitenden Handel mit Waren und Dienstleistungen initiiert werden ${ }^{150}$ - und zwar dann, wenn Inländer durch die Einfuhr von Gütern auch die unterschiedlichen nationalen Regulierungen ,importieren“, denen die Produktion der Güter unterliegt, wenn also im Heimatland des Käufers das Ursprungslandprinzp gilt. Im Unterschied zum ökonomischen Wettbewerb findet damit im Systemwettbewerb neben Wahlakten innerhalb eines Ordnungsrahmens (,choice within rules“) auch eine Entscheidung über diesen Rahmen selbst statt (,choice of rules"). ${ }^{151}$ Ergeben sich durch Abwanderung oder Kaufkraftverlagerung nachteilige Effekte für die Volkswirtschaft, die sich auf die

\footnotetext{
${ }^{147} \mathrm{Vgl}$. im Folgenden Schaub (2001), S. 59ff.

148 Vgl. im Folgenden Seliger/Herrmann-Pillath (2000), S. 1150.

${ }^{149} \mathrm{Vgl}$. Seliger/Herrmann-Pillath (2000), S. 1151.

${ }^{150} \mathrm{Vgl}$. Streit/Mussler (1995), S. 77.

Is1 Vgl. Wohlgemuth (1998), S. 70.
} 
Wiederwahlchancen der Regierungspartei auswirken, entsteht daraus ein Anreiz für die politisch Handelnden, die bestehenden Institutionen kritisch zu betrachten. ${ }^{152}$ Dies kann einen Prozess hervorrufen, in dem das bestehende System auf Mängel untersucht wird und eine bessere Anpassung an die Präferenzen der Bürger erfolgt (Parallelprozess ${ }^{153}$ ). Dabei können entweder in anderen Staaten bestehende und bewährte Regelungen imitiert oder völlig neue Arrangements im nationalen System (,Innovation“) entwickelt werden. Auf diese Weise erfolgt durch den Systemwettbewerb eine zusătzliche Kontrolle des Handelns der politischen Akteure.

Im Systemwettbewerb wird jedoch auch die Gefahr gesehen, dass die (drohende) Abwanderung von Bürgern die Nationalstaaten dazu zwingt, nicht nur das Niveau ihrer Steuern, sondern auch das ihrer Sozial- und Umweltregulierungen nach unten anzupassen, um finanziell handlungsfähig zu bleiben. ${ }^{154}$ Die zugrunde liegenden Modelle basieren allerdings auf sehr restriktiven Annahmen, wie z.B. einem pareto-optimalen Status quo der nationalen Politik, vollkommen mobilen Bürgern, die auf bekannte und als gegeben vorausgesetzte Kostendifferenzen sofort mit Abwanderung reagieren sowie einer vollkommen friktionslosen Anpassung der Politiker an sich ändernde Bedingungen. ${ }^{155} \mathrm{Da}$ zudem unberücksichtigt bleibt, dass zum einen Politiker nicht allwissend sind und die passenden Lösungen für anstehende Probleme meist nicht kennen, zum anderen die Bürger Europas unterschiedliche und veränderliche Präferenzen besitzen, deren Durchsetzung mit Hilfe von Wahlen nur eingeschränkt möglich ist, können in diesen Modellen die Potentiale des institutionellen Wettbewerbs, der als Entdeckungsverfahrens für alternative Problemlösungen und als Mittel zur Präferenzoffenbarung und -durchsetzung dienen kann, nicht zum Tragen kommen.

Alternativen zu einem Systemwettbewerb sind entweder wettbewerbsbeschränkende Maßnahmen - beispielsweise in Form von Einschränkungen der Faktormobilităt oder eine Vorab-Harmonisierung der Systeme. ${ }^{156}$ Die erste Möglichkeit widerspricht der Konzeption Europas als Binnenmarkt ohne Grenzen, in dem die vier Grundfreiheiten gelten und die Mitgliedstaaten auf ein System verpflichtet sind, „das den Wettbewerb innerhalb des Binnenmarkts vor Verfälschungen schützt“157. Die Möglichkeit einer Vorab-Harmonisierung der Sozialsysteme wurde zu Beginn des europäischen Einigungsprozesses im Wesentlichen im Hinblick auf die Sicherstellung der Freizügigkeit von Arbeitnehmern zwar diskutiert, jedoch bald aufgrund der praktischen Schwierigkeiten aufgegeben, historisch gewachsene und sehr heterogene Systeme umzugestalten. Inzwischen schließt der EGV eine solche Harmonisierung für die nationalen Gesundheitssysteme in Art. 152 Abs. 5 aus, indem er die alleinige Kompetenz für

\footnotetext{
${ }^{152} \mathrm{Vgl}$. Wohlgemuth (1998), S. 73ff.

${ }^{153}$ Nach Hoppmann (1967), S. 88ff, lässt sich der ökonomische Wettbewerbsprozess in zwei Elemente zerlegen: einen Austauschprozess zwischen Anbietern und Nachfragern und einen Parallelprozess zwischen den konkurrierenden Anbietern in Reaktion auf die Nachfrageentscheidung. Vgl. auch Wohlgemuth (1998), S. 73f.

${ }^{154}$ Vgl. z.B. Sinn (1994), S. 89ff.

${ }^{155}$ Vgl. Wohlgemuth (1998), S. 77.

${ }^{156} \mathrm{Vgl}$. Wohlgemuth (1998), S. $78 \mathrm{f}$.

${ }^{157}$ Art 3 Abs. $1 \mathrm{~g} \mathrm{EGV.}$
} 
die Organisation des Gesundheitswesens und die medizinische Versorgung den Mitgliedstaaten zuweist. Dadurch bleibt die Vielfalt der Gesundheitssysteme in Europa erhalten, wodurch sich theoretisch ein institutioneller Wettbewerb im Bereich der Gesundheitsversorgung entwickeln kann.

Abbildung 11: Gesundheitsquoten ${ }^{1)}$ im europäischen Vergleich 2002 (in Prozent des BIP)
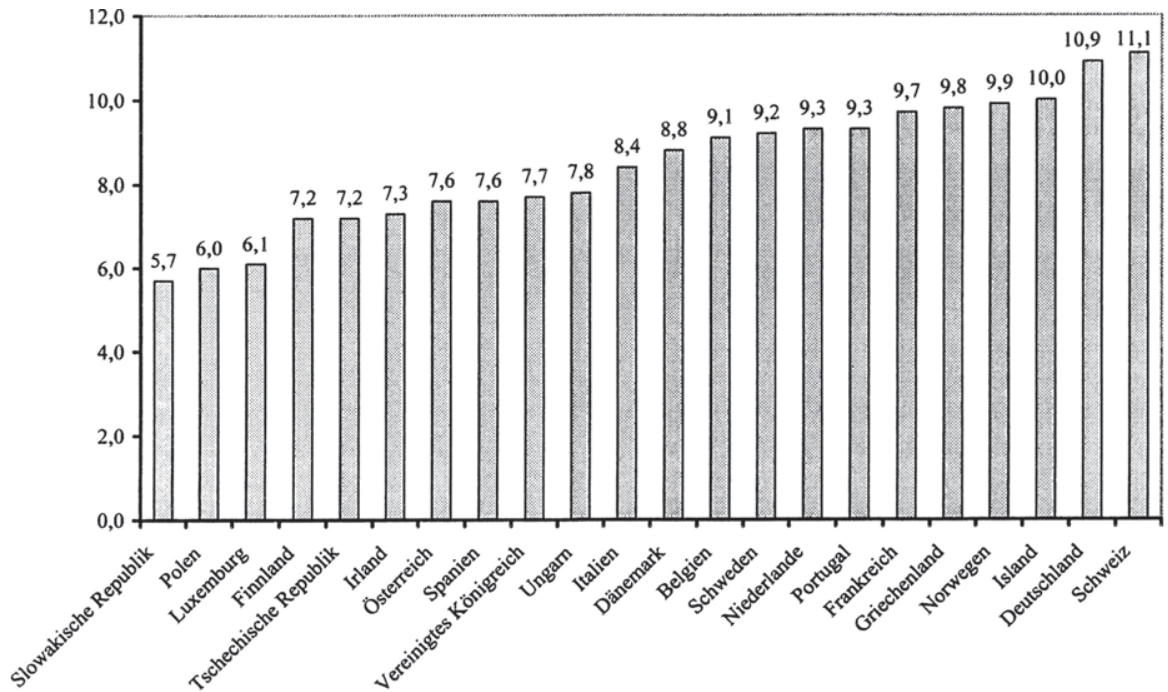

1) Gesamte Gesundheitsausgaben eines Landes in Prozent des jeweiligen Bruttoinlandsprodukts

Quelle: Organisation for Economic Co-operation and Development (OECD) (2005)

Die Schwierigkeiten, die mit einer Harmonisierung des Gesundheitsschutzes in der EU verbunden wären, macht ein Blick auf die Gesundheitsquoten ${ }^{158}$ der Mitgliedstaaten deutlich (vgl. Abbildung 11). In Deutschland betrug diese Quote im Jahre 2002 10,9 Prozent, während sie beispielsweise in Finnland, Irland, Österreich, Spanien und Großbritannien unter 8 Prozent blieb und in Polen und der Slowakei sogar nur bei 6,0 bzw. 5,7 Prozent lag. Eine Harmonisierung dieser Quote auf einem niedrigen Niveau brächte für Länder wie Deutschland und Frankreich erhebliche Einschränkungen für die Gesundheitsversorgung der Bevölkerung mit sich, die nicht ohne weiteres hingenommen würden. ${ }^{159}$ Umgekehrt kämen bei einer Erhöhung der Gesundheitsquote auf das deutsche Niveau auf die neuen Mitgliedstaaten, aber auch auf Länder wie Finnland und Irland erhebliche Mehrausgaben zu, die durch Steuer- oder Beitragssatzerhöhungen finanziert werden müssten und so die Wettbewerbsfähigkeit dieser Länder ver-

${ }^{158}$ Die Gesundheitsquote setzt die Gesundheitsausgaben eines Landes in Relation zum entsprechenden Bruttoinlandsprodukt.

${ }^{159} \mathrm{Vgl}$. dazu auch Wille (2005), S. 44. 
schlechterten. Darum ist eine solche Angleichung der Gesundheitsquoten aus gesamtwirtschaftlicher Sicht nicht sinnvoll.

Zudem können diese stark divergierenden Gesundheitsquoten auch Ausdruck der unterschiedlichen Präferenzen der Bevölkerung in den verschiedenen europäischen Staaten bezüglich der Ausgestaltung des jeweiligen Gesundheitssystems sein. In diesem Zusammenhang zeigt Abbildung 12, dass die Zufriedenheit der EU-Bürger mit ihrem jeweiligen Gesundheitswesen nicht eindeutig mit dem Anteil der öffentlichen Gesundheitsausgaben am Bruttoinlandsprodukt (BIP) korreliert. So sind z.B. die Einwohner Österreichs, Finnlands und Luxemburgs deutlich zufriedener mit ihrem System als die deutsche Bevölkerung, obwohl Deutschland eine um mindestens zwei Prozentpunkte höhere öffentliche Gesundheitsquote aufweist.

Abbildung 12: Zusammenhang zwischen dem Anteil der öffentlichen Gesundheitsausgaben am BIP ${ }^{1}$ und der Bewertung der Gesundheitssysteme ${ }^{2}$

Bewertung des Gesundheitssystems

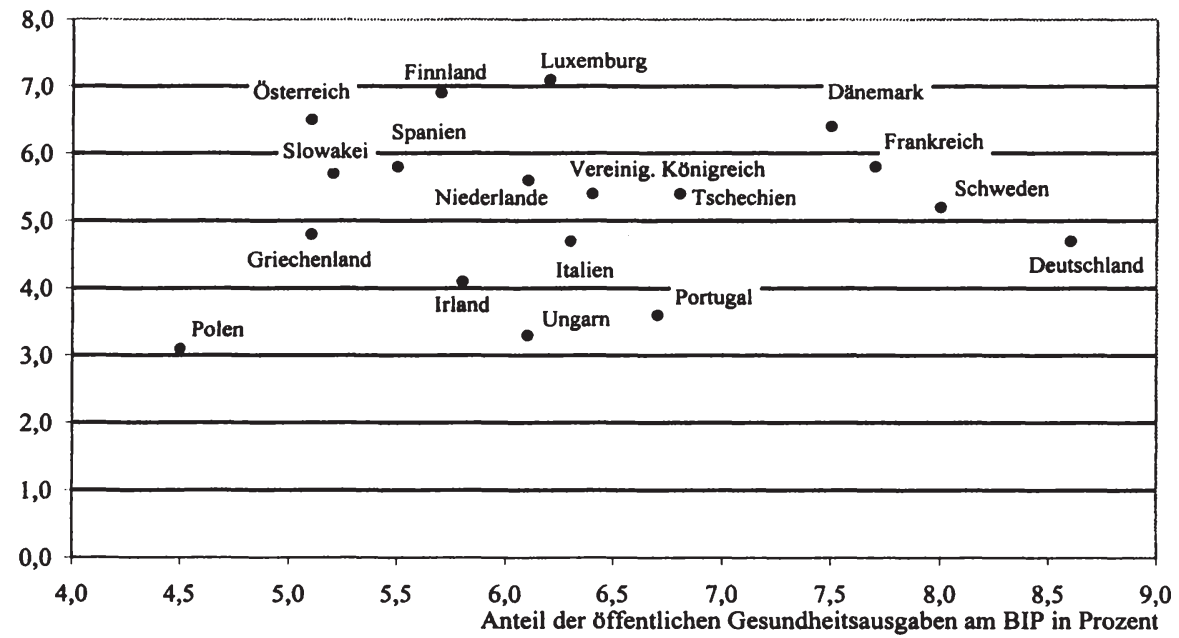

' Daten für 2003, für das Vereinigte Königreich von 2002; Schätzwerte für Frankreich, Griechenland und Ungarn

${ }^{2}$ Daten von 2004, Mittelwert auf einer Skala von 0 (= außerst schlecht) bis 10 (= äußerst gut)

Quelle: Statistisches Bundesamt (Hrsg.) (2006); Organisation for Economic Cooperation and Development (OECD) (2005); eigene Berechnungen

Abbildung 13 zeigt zudem, dass die Zufriedenheit der EU-Bürger mit ihrem jeweiligen Gesundheitswesen auch nicht damit korreliert, wie hoch der Finanzierungsanteil der Sozialversicherung an den gesamten Gesundheitsausgaben ist, ob es sich also um ein 
überwiegend steuer- oder beitragsfinanziertes System handelt. ${ }^{160}$ Diese Beobachtung kann dahingehend interpretiert werden, dass die Präferenzen der EU-Bürger hinsichtlich der Finanzierung ihres Gesundheitssystems nicht homogen sind, was ein Nebeneinander unterschiedlicher Ausgestaltungen rechtfertigt. Es ist daher wahrscheinlich, dass ein funktionierender Systemwettbewerb nicht zu einer vollkommenen Harmonisierung der Gesundheitssysteme führen würde.

Abbildung 13: Korrelation zwischen dem Finanzierungsanteil der Sozialversicherung an den gesamten Gesundheitsausgaben und der Zufriedenheit der Bürger mit ihrem Gesundheitssystem

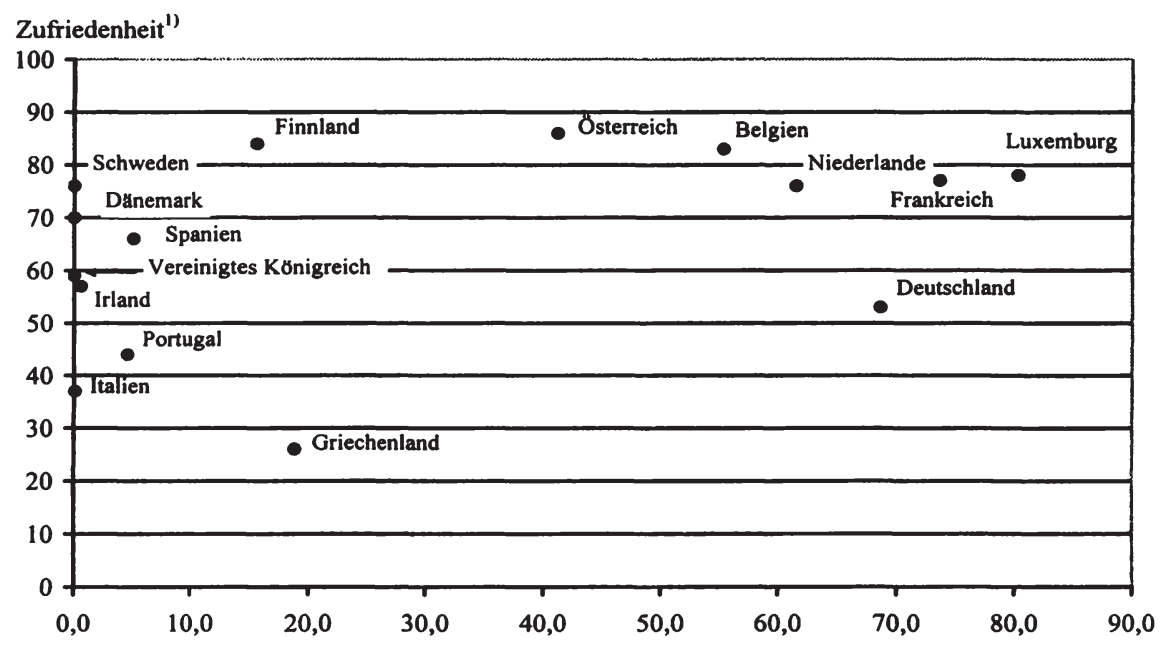

Finanzierungsanteil der Sozialversicherung an den gesamten Gesundheitsaufgaben in Prozent

1) Die Daten zur Zufriedenheit geben an, wie viel Prozent der Bürger des betreffenden Landes sehr zufrieden oder zufrieden mit ihrem Gesundheitssystem sind.

Quelle: World Health Organization (WHO) (2005); Statistisches Bundesamt (Hrsg.) (2004); eigene Berechnungen

Voraussetzung für einen funktionierenden institutionellen Wettbewerb ist die Freiheit des Waren-, Dienstleistungs-, Personen- und Kapitalverkehrs sowie die Anwendung des Ursprungslandprinzips, damit beim Import von Gütern auch implizit die Regulierungen eingeführt werden, unter denen die Herstellung erfolgt. Durch die Gültigkeit der Waren- und Dienstleistungsfreiheit für Gesundheitsleistungen ist eine Inanspruchnahme (mit Ausnahme von Krankenhausleistungen) in jedem EU-Staat möglich geworden, d.h. das Territorialprinzip im Gesundheitssystem wurde durch die Rechtsprechung des EuGH in Bezug auf die Leistungsinanspruchnahme in weiten Bereichen

${ }^{160}$ Eine Übersicht über die Aufteilung der Finanzierung auf Sozialversicherungen, sonstige staatliche Gebietskörperschaften und private Zahlungen gibt Tabelle VI im Anhang C. 
abgeschwächt. ${ }^{161}$ Es ist jedoch nicht vollständig aufgehoben, da beispielsweise bei einer Inanspruchnahme zu Lasten der gesetzlichen Krankenkassen der deutsche Leistungskatalog für eine Kostenerstattung maßgeblich bleibt. In Bezug auf die Versicherungszugehörigkeit gilt das Territorialprinzip weiterhin uneingeschänkt, so dass die Wahl eines sozialen Sicherungssystems nicht ohne weiteres möglich ist. Die Mitgliedschaft in einem sozialen Krankenversicherungssystem ist i.d.R. an den Ort der Beschäftigung bzw. den Wohnort geknüpft, so dass ein Bürger, der mit der Ausgestaltung des nationalen Gesundheitswesen nicht einverstanden ist, nicht die Möglichkeit besitzt, aus diesem System abzuwandern - es sei denn, er wechselt auch das Land seines Arbeitsplatzes bzw. Wohnsitzes. Eine Abwanderung aus dem Gesundheitssystem ist darum derzeit fur die meisten Versicherten nur in Form einer Verlagerung der Nachfrage nach Gesundheitsleistungen in andere EU-Mitgliedstaaten möglich, so dass der Patient nur den Ort der Leistungsinanspruchnahme frei wählen kann, den Leistungskatalog aber nicht. Dennoch können sich auch im Status quo Auswirkungen der grenzüberschreitenden Nachfrage nach Gesundheitsleistungen auf die Elemente der Gesundheitssysteme ergeben, weil die Patienten und Versicherten auf diese Weise die Möglichkeit besitzen, die Folgen nationalstaatlicher Regulierungen zu umgehen, ohne dafür ihr Wohn- bzw. Beschäftigungsland wechseln zu müssen. So können die Versicherten durch die Behandlung im Ausland beispielsweise nationale Wartelisten umgehen, die aufgrund fehlender Kapazitäten oder einer zu geringen Zahl von Leistungserbringern entstehen. Vorangetrieben werden könnte der Wettbewerb der unterschiedlichen Gesundheitssysteme im Sinne eines Entdeckungsverfahrens für geeignete institutionelle Problemlösungsmöglichkeiten durch die in Abschnitt 2.5 vorgestellte offene Methode der Koordinierung, die u.a. den Informationsaustausch der Mitgliedstaaten über die von ihnen gewählten Regulierungsmaßnahmen verbessern und im Rahmen eines Benchmark-Verfahrens Aufschluss über deren Erfolge geben soll. Dabei ist jedoch zu berücksichtigen, dass der Systemwettbewerb dazu dienen soll, die nationalen Systeme stärker an den Präferenzen der jeweiligen Bürger auszurichten. Stellt sich in Zukunft heraus, dass die OMK letztlich dazu eingesetzt wird, den europäischen Institutionen einen größeren Einfluss auf die Ausgestaltung der Systeme zu verleihen, ohne dass die nationalen Akteure auf den verschiedensten Ebenen die eigentlich Handelnden bleiben, entspräche diese Vorgehensweise einer Harmonisierung nach den Vorstellungen der EU, die der Konzeption eines dezentralen Wettbewerbs der Systeme entgegenstünde.

\footnotetext{
${ }^{161}$ Vgl. im Folgenden auch Oberender (2001), S. 85f.
} 


\section{Fazit}

Die deutsche Gesundheitspolitik reagierte auf die EuGH-Urteile in den Rechtssachen „Kohll" und „Decker“ mit der Befürchtung, dass eine Übertragung der Urteile auf Deutschland die Finanzierbarkeit des gesamten Gesundheitssystems in Fragen stellen würde. ${ }^{162}$ Diese Befürchtung hat sich inzwischen als überzogen erwiesen. Die Finanzierungsprobleme der GKV stehen in keinem Zusammenhang mit der Europäisierung des SGB V durch das GMG im Jahre 2004. Die Reformversuche der letzten Jahre haben dabei gezeigt, dass die deutsche Gesundheitspolitik nicht in der Lage ist, die gesetzliche Krankenversicherung auf der Finanzierungs- oder Ausgabenseite grundlegend und zukunftssicher zu verändern. Die ergriffenen Maßnahmen stellen eine Reaktion auf die durch die ungünstigen Anreizstrukturen hervorgerufenen Fehlallokationen im System dar. Oftmals werden dabei Versuche einer Ergebniskorrektur unternommen, indem in das bestehende, bereits überregulierte System weitere zentrale Steuerungselemente eingefügt werden. Aus ökonomischer Sicht ist daher die Öffnung der europäischen Gesundheitsmärkte zu begrüßen. Die wettbewerblichen Einflüsse, die die europäischen Grundfreiheiten entfalten, können langfristig zu einer Beseitigung von Ineffizienzen beitragen, die aufgrund der Überregulierung des Systems existieren, und sich auf diese Weise positiv auf die Finanzierbarkeit und die Qualität der Gesundheitsversorgung auswirken. Derzeit steckt diese Entwicklung noch in den Anfängen, so dass sich diese Auswirkungen nur langfristig zeigen können.

Gegenwärtig ist den betrachteten Leistungsbereichen eine gemeinsame Tendenz festzustellen: Durch die zunehmende Europäisierung des deutsche Gesundheitswesen ergeben sich momentan keine signifikanten Steigerungen der Leistungsausgaben der gesetzlichen Krankenkassen. Sollte sich der Einfluss der europäischen Grundfreiheiten jedoch verstärken, entweder indirekt durch eine wachsende Nachfrage nach Leistungen im Ausland oder direkt durch weitere Urteile des Europäischen Gerichtshofs, verlieren die Steuerungselemente des Systems zunehmend ihre Wirksamkeit. Es ist davon auszugehen, dass dies ohne geeignete Reformen des Systems zu steigenden Ausgaben der gesetzlichen Krankenkassen führen würde.

Ergäbe sich daraus eine Gefahr für die finanzielle Stabilität des Systems, rechtfertigt dies grundsätzlich eine Begrenzung der Leistungsinanspruchnahme im Ausland und damit der europäischen Grundfreiheiten, die der EuGH in seinen bisherigen Urteilen anerkannt hat. Dabei sollte jedoch berücksichtigt werden, dass nach der ständigen Rechtsprechung des Gerichtshofs gewährleistet sein muss, dass die ergriffenen Maßnahmen nicht über das hinausgehen, was zu diesem Zweck objektiv notwendig ist, und dass das gleiche Ergebnis nicht durch Regelungen erreicht werden kann, die die europäischen Grundfreiheiten weniger stark einschränken ${ }^{163}$ In diesem Sinne wäre es aus ökonomischer Sicht sinnvoll, die territorialen Begrenzungen, die im Gesundheitswesen beispielsweise bei der vertragsärztlichen Vergütung bestehen, aufzuheben und Regelungen zu finden, die eine unterschiedslose Berücksichtigung des inländischen und

${ }^{162} \mathrm{Vgl}$. o.V. (1998b), S. 36ff.

${ }^{163} \mathrm{Vgl}$. Europäischer Gerichtshof (2003e), Rnr. 68; Europäischer Gerichtshof (2001a), Rnr. 75. 
ausländischen Leistungsgeschehens ermöglichen. Dabei zeigt sich sektorübergreifend, dass dies umso leichter gelingt, je weniger staatliche Regulierungen existieren und je mehr die Steuerung von Angebot und Nachfrage dem Markt überlassen bleibt. Die adäquate Reaktion auf eine zunehmende Europäisierung des Gesundheitswesens stellt darum nicht die Abschottung des gesamten Systems, sondern seine Deregulierung dar. Wie die Entwicklung im Bereich der Vorsorge- und Rehabilitationseinrichtungen zeigt, der bereits heute in vergleichsweise geringem Ausmaß von einer zentralen Steuerung des Angebots betroffen ist, kann sich daraus ein grenzüberschreitender Wettbewerb entwickeln, der die inländischen Anbieter aufgrund ihrer höheren Preise unter Druck setzt. Auf diese Weise können bestehende Rationalisierungsreserven in der Gesundheitsversorgung identifiziert werden und zu einer Senkung der Kosten in den entsprechenden Bereichen beitragen. Da ein reiner Preiswettbewerb angesichts der Konkurrenz mit Anbietern in Osteuropa jedoch keine wünschenswerte Option darstellt, können sich die inländischen Leistungserbringer einen Wettbewerbsvorteil durch eine vergleichsweise hohe Qualität ihrer Leistungen erarbeiten. Prinzipiell bietet eine Öffnung der europäischen Grenzen für Gesundheitsleistungen den inländischen Leistungsanbietern damit auch die Chance, durch qualitativ hochwertige Leistungen ausländische Patienten $\mathrm{zu}$ attrahieren.

Bereits heute arbeiten die gesetzlichen Krankenkassen in verschiedenen Bereichen der Gesundheitsversorgung mit ausländischen Anbietern zusammen. Der einzelnen Krankenkasse bietet die erweiterte Möglichkeit zur Kostenübernahme im Ausland neben den Einsparmöglichkeiten durch geringere Preise eine zusätzliche Chance: Sie kann ihren Service verbessern und die Zufriedenheit ihrer Kunden erhöhen, indem sie verlässliche Informationen über die bestehenden Möglichkeiten der Leistungsinanspruchnahme im Ausland bereitstellt. Eine Mitgliederbefragung der Techniker Krankenkasse (TK) ergab in diesem Zusammenhang, dass mangelnde Informationen über geeignete Ärzte und Krankenhäuser für die Versicherten eines der wichtigsten Hindernisse für eine Behandlung im Ausland darstellen. ${ }^{164}$ Dabei können die Krankenkassen durch den Abschluss von Verträgen mit ausländischen Leistungserbringern ihr Angebot um interessante Optionen erweitern. Diese Strategie verfolgt eine Reihe von Krankenkassen bereits im Bereich der Vorsorgemaßnahmen und bei Arzneimitteln. So hat die Hanseatische Krankenkasse als erste deutsche Krankenkasse zu Beginn des Jahres 2005 mit entsprechenden Einrichtungen im Kurort Marienbad (Tschechien) Verträge über die Versorgung ihrer Versicherten mit Vorsorge- und Rehabilitationsmaßnahmen abgeschlossen. ${ }^{165}$ Verschiedene andere Kassen folgten seither diesem Beispiel. Bereits im Jahre 2004 schloss die AOK Rheinland-Pfalz einen Vertrag mit der holländischen Versandapotheke Doc Morris. ${ }^{166}$ Diese Verträge verbessern und erweitern das Angebot der deutschen Kassen und sind ein erster Schritt zu einem europäischen Wettbewerb um die Wirtschaftlichkeit und die Qualität der Gesundheitsversorgung. ${ }^{167}$ Wünschenswert wäre jedoch auch, dass die Kassen die Patienten - unabhängig von beste-

\footnotetext{
${ }^{164} \mathrm{Vgl}$. Techniker Krankenkasse (TK) (2003), S. 16.

${ }^{165} \mathrm{Vgl}$. o.V. (2005e).

${ }^{166} \mathrm{Vgl}$. Westdeutscher Rundfunk (WDR) (2004).

${ }^{167}$ So auch Greß/Axer/Wasem (2003), S. 91.
} 
henden Verträgen - vor möglichen Risiken warnen und ihnen bei der Auswahl seriöser Leistungsanbieter helfen. Zudem sollten die Patienten im Vorfeld der Behandlung darüber informiert werden, welche Leistungen erstattungsfähig sind, um zu verhindern, dass unbeabsichtigt Behandlungen durchgeführt werden, für die die Versicherten dann die Kosten selbst tragen müssten.

Bei der grenzüberschreitenden Inanspruchnahme durch deutsche Patienten spielt die Qualität der Leistungen eine große Rolle. Da diese durch Vergütungsanreize im Rahmen der Kostenerstattung allein nicht sichergestellt werden kann, ist in diesem Bereich eine Kontrolle durch eine übergeordnete Aufsichtsstelle unerlässlich. Daneben bietet eine vertragliche Bindung ausländischer Leistungserbringer den Vorteil, dass neben dem Honorar auch qualitätssichernde Maßnahmen vereinbart werden können. Es ist jedoch davon auszugehen, dass die Qualität medizinischer Leistungen nicht nur international, sondern auch national schwankt. Generell zu unterstellen, dass eine Behandlung im Ausland für die Patienten mit größeren Gefahren verbunden sei als im Inland, wäre darum ein Versuch, das deutsche Gesundheitswesen gegen europäische Einflüsse abzuschotten, der angesichts des Zusammenwachsens der europäischen Staaten anachronistisch erscheint. Dennoch ist es wünschenswert, dass eine Qualitätskontrolle generell europaweit möglich wird und die Rechte der Versicherten bezüglich einer grenzüberschreitenden Durchsetzung von Haftungsansprüchen eine deutliche Stärkung erfahren, um dieses Hindernis für eine grenzüberschreitende Inanspruchnahme von Leistungen abzubauen.

Alle Maßnahmen zur Information und Qualitätssicherung zielen darauf ab, die Mobilität der Patienten zu erhöhen, was Voraussetzung für einen zunehmenden grenzüberschreitenden Wettbewerb in der Gesundheitsversorgung ist, der sich in einzelnen Bereichen, wie z.B. bei Kuren und Arzneimitteln, heute schon zeigt und letztlich in Gestalt eines Wettbewerbs der Systeme auf die Ausgestaltung des deutschen Gesundheitswesens Einfluss nehmen kann. In diesem Zusammenhang ist auch der Versuch der europäischen Institutionen zu berücksichtigen, einen größeren Einfluss auf die Organisation der nationalen Gesundheitssysteme zu gewinnen. Solche Maßnahmen sind dann wünschenswert, wenn sie zu einem Abbau wettbewerbsbehindernder nationalstaatlicher Regulierungen beitragen. Darum sind die Initiativen der Europäischen Union bezüglich einer besseren Information der Versicherten und Patienten sowie die Maßnahmen zur Unterstützung eines Erfahrungsaustauschs der Mitgliedstaaten bezüglich möglicher Lösungsstrategien für länderübergreifend bestehenden Probleme der Gesundheitssysteme - z.B. im Rahmen der OMK - grundsätzlich zu begrüßen. Allerdings darf dabei keine Harmonisierung der Gesundheitsversorgung auf einem einheitlichen europäischen Niveau angestrebt werden. Darüber hinaus müssen die Kompetenzen in diesem Bereich bezüglich der Auswahl konkret zu ergreifender Maßnahmen bei den nationalen Regierungen verbleiben. Ihnen obliegt auch weiterhin die Aufgabe, die vorhandenen Strukturprobleme zu erkennen und entsprechende Änderungen durchzusetzen. Die Europäisierung der Gesundheitssysteme lässt ordnungspolitische Fehlentscheidungen der vergangenen Jahrzehnte offenbar werden. Sie erzwingt jedoch keine weit reichende Liberalisierung der Gesundheitsversorgung. Dies ist und bleibt Aufgabe der nationalen Gesundheitspolitik. 


\section{Anhang}

\section{Anhang A: Ziele der OMK}

Tabelle I: Vorschlag für gemeinsame Ziele im Bereich Gesundheitswesen und Langzeitpflege (OMK)

\section{Ziel: Sicherung des Zugangs zur Gesundheitsversorgung}

Hochwertiges und bedarfsgerechtes Versorgungsangebot für die gesamte Bevölkerung, insbesondere für Personen die dauernde Pflege bedürfen, Personen und Gruppen mit besonderen Zugangsschwierigkeiten sowie Personen mit niedrigem Einkommen.

Sicherung der Zugänglichkeit für behinderte Personen

Angemessenes Betreuungsangebot für ältere Menschen, insbesondere durch verstärkte Koordination zwischen Sozialfürsorge, Grundversorgung, Krankenhausversorgung und Facheinrichtungen

Förderung der Instrumente für die Palliativmedizin und Sterbebegleitung

Verringerung der regionalen Unausgewogenheit des Versorgungsangebots

Entwicklung angemessener, mit qualifiziertem Personal ausgestatteter Strukturen, um das Angebot zu verbessern und die Fristen für den Zugang zur Behandlung zu verkürzen,

Förderung einer Personalverwaltung, durch die sich die Folgen der Bevölkerungsalterung im Sektor Gesundheitsversorgung und Langzeitpflege auffangen lassen.

\section{Ziel: Förderung einer hochwertigen Versorgung}

Verfahren der Kosten-Nutzen-Analyse stärker anwenden,

Berücksichtigung der geschlechtsspezifischen Unterschiede bei der Festlegung der Präventiv- und Gesundheitspolitik,

Sicherung des Ausbildungsniveaus der Fachkräfte,

Ausbau der Maßnahmen für Gesundheit und Sicherheit am Arbeitsplatz,

Aufteilung der Ressourcen auf Regionen, Dienste und verschiedene Versorgungsarten nach dem tatsächlichen Bedarf,

Förderung einer Verwaltung, die die Anpassungsfähigkeit der Systeme an den Bedarf begünstigt,

Festlegung der Rechte der Patienten und ihrer Familien sowie die Modalitäten für die Mitwirkung der organisierten Zivilgesellschaft. 


\section{Ziel: Sicherung der langfristigen Finanzierbarkeit}

Entwicklung der systematischen Prävention und Beratung bei Eintritt in die Versorgungskette,

Erreichen einer tragbaren Ausgabenentwicklung durch Maßnahmen, die den Besonderheiten der einzelnen Systeme Rechnung tragen,

Sicherung einer angemessenen Finanzierung des Systems, um auf die neuen Bedürfnisse in Zusammenhang mit Alterung, gesellschaftlichem Wandel und technischem Fortschritt einzugehen,

Angebot von Gesundheitsleistungen mit einem möglichst günstigen Kosten-NutzenVerhältnis,

besserer Wirkungsgrad des Systems z.B. durch Dezentralisierung

Quelle: Europäische Kommission (2004b), S. 8ff, eigene Darstellung. 
Tabelle II: Ziele für die gestraffte OMK in den Bereichen „Soziale Eingliederung“, „Renten“ und „Gesundheitsversorgung und Langzeitpflege“"

\section{Übergreifende Ziele der OMK für Sozialschutz und soziale Eingliederung:}

Den sozialen Zusammenhalt und die Chancengleichheit für alle fördern durch angemessene, leicht zugängliche, finanziell nachhaltige, anpassungsfähige und effiziente Sozialschutzsysteme und Prozesse der sozialen Eingliederung.

Enge Wechselwirkung mit den Lissabonner Zielen - das Wirtschaftswachstum zu stärken und mehr und bessere Arbeitsplätze zu schaffen - und mit der EU-Strategie der nachhaltigen Entwicklung.

Die Governance, Transparenz und Einbeziehung von Stakeholdern bei Gestaltung, Durchfuhrung und Monitoring der Politik verbessern.

\section{Ziele in den verschiedenen Teilbereichen:}

Die Beseitigung von Armut und sozialer Ausgrenzung entscheidend voran-bringen:

Die aktive soziale Eingliederung aller sicherstellen durch Förderung der Erwerbsbeteiligung und Bekämpfung von Armut und Ausgrenzung der am stärksten an den Rand der Gesellschaft gedrängten Menschen und Gruppen.

Sicherstellen, dass alle zu den grundlegenden Ressourcen, Rechten und Dienstleistungen Zugang haben, der Voraussetzung ist für die gesellschaftliche Teilhabe. Dabei ist gegen extreme Formen der Ausgrenzung und alle Formen von zu Ausgrenzung führender Diskriminierung vorzugehen.

Dafür Sorge tragen, dass die Politik der sozialen Eingliederung gut koordiniert ist und alle Regierungsebenen und Akteure einbezieht, einschließlich der von Armut Betroffenen. Das effiziente und wirksame Mainstreaming der Eingliederungspolitik in allen relevanten Bereichen der öffentlichen Politik gewährleisten, einschließlich der Wirtschafts- und Haushaltspolitik und der Strukturfondsprogramme (vor allem ESF). Dies gilt auch für das Gender-Mainstreaming. 


\section{Eine angemessene und tragfăhige Altersversorgung sicherstellen:}

Im Geist der Solidarität und Generationengerechtigkeit ein angemessenes Renteneinkommen für alle und den Zugang zu Renten gewährleisten, die es den Menschen erlauben, nach der Verrentung einen angemessenen Lebensstandard zu bewahren.

Im Rahmen des Erfordernisses solider öffentlicher Finanzen die Zukunftssicherheit öffentlicher und privater Rentensysteme sicherstellen, vor allem durch: Verlängerung des Arbeitslebens und Förderung des aktiven Alterns, Ausgewogenheit von Beiträgen und Leistungen, Förderung der Erschwinglichkeit und Sicherheit kapitalgedeckter und privater Systeme.

Gewährleisten, dass die Rentensysteme transparent und an die Bedürfnisse und Erwartungen von Frauen und Männern, die Anforderungen der modernen Gesellschaft und die Zwänge der demografischen Alterung und des Strukturwandels angepasst sind. Sicherstellen, dass die Menschen die zur Ruhestandsplanung erforderlichen Informationen erhalten, und dass Reformen auf der Basis eines möglichst breiten Grundkonsenses durchgeführt werden.

Eine für alle zugängliche, qualitativ hochwertige und nachhaltige Gesundheitsversorgung und Langzeitpflege sicherstellen:

Den Zugang aller zu einer angemessenen Gesundheitsversorgung und Langzeitpflege sicherstellen und dabei gewährleisten, dass Pflegebedürttigkeit nicht zu Armut und finanzieller Abhängigkeit führt. Gegen Ungleichheiten beim Zugang zu Pflege und bei den Gesundheitsergebnissen vorgehen.

Die Qualität der Gesundheitsversorgung und der Langzeitpflege fördern und die Pflegesysteme an die sich wandelnden Erfordernisse und Präferenzen der Gesellschaft und des Einzelnen anpassen, insbesondere durch Verwirklichung von Qualitätsstandards, die der internationalen Best Practice entsprechen, und durch Stärkung der Verantwortlichkeit der Angehörigen der Gesundheitsberufe sowie der Patienten und der Pflegeempfänger.

Sicherstellen, dass eine angemessene und qualitativ hochwertige Langzeitpflege erschwinglich und nachhaltig bleibt. $\mathrm{Zu}$ diesem Zweck eine gesunde und aktive Lebensweise, eine gute Personalausstattung des Pflegesektors und eine rationelle Ressourcennutzung fördern, vor allem durch geeignete Anreize für Nutzer und Anbieter sowie eine gute Governance und Koordination zwischen den Pflegesystemen und den Pflegeeinrichtungen.

Quelle: Europäische Kommission (2005e), S. 6f, eigene Darstellung. 


\section{Anhang B: Daten zur Patientenmobilität}

Tabelle III: Zahl der Staatsangehörigen eines anderen Mitgliedstaates, die als Patienten im jeweiligen Mitgliedstaat behandelt wurden

\begin{tabular}{|c|c|}
\hline Belgien & $\begin{array}{l}\text { Die belgischen Behörden haben Informationen in Form von Finanz- } \\
\text { summen übermittelt. } \\
\text { Für das Jahr 2000: } \\
\text { Ausgaben der ausländischen Versicherten in Belgien im Bereich ab- } \\
\text { hängig Beschäftigte: } 6.683 .209 .508 \mathrm{FB}(165.672 .436 €) \text {, Steigerung } \\
\text { zum Vorjahr: } 6 \% \\
\text { Ausgaben der ausländischen Versicherten in Belgien im Bereich Selb- } \\
\text { ständige: } 125.797 .373 \mathrm{FB}(3.118 .435 €) \text {, Steigerung } 7,94 \% \text { im Ver- } \\
\text { gleich zum Vorjahr, jedoch bis zu diesem Zeitpunkt konstant rückläu- } \\
\text { fig. } \\
\text { Höhe der belgischen Forderungen insgesamt gegenüber anderen Mit- } \\
\text { gliedstaaten für die Kategorie der Personen, die berechtigt sind, sich in } \\
\text { Belgien im Rahmen des Vordrucks E-112 behandeln zu lassen: } \\
25.907 .697,17 €(1.045 .113 .913 \mathrm{FB}) \text {, dies entspricht } 14.061 \text { Personen } \\
\text { darunter: Niederlande: } 453.320 .575 € \text {, dies entspricht } 6.262 \text { Personen }\end{array}$ \\
\hline Luxemburg & 242.124.142 $€$, dies entspricht 3.551 Personen \\
\hline Italien & $210.655 .435 €$, dies entspricht 2.832 Personen. \\
\hline Dänemark & 2001: 2.401 Personen, darunter 1.130 aus Deutschland. \\
\hline Spanien & $\begin{array}{l}\text { 2001: } \\
\text { E-112: } 457.821,9 € \text {, dies entspricht 3.156 Personen } \\
\text { E-111 20.102.004,2 } € \text {, dies entspricht 133.958 Personen } \\
\text { Gesamt: } 20.559 .825 € \text {, dies entspricht } 137.114 \text { Personen }\end{array}$ \\
\hline Frankreich & $\begin{array}{l}\text { 2001: } 435.856 \text { Personen } \\
\text { 2002: Forderungen: 297,2 Millionen } €\end{array}$ \\
\hline Italien & 19991.022 Personen. \\
\hline Irland & $\begin{array}{l}\text { In Irland wurde in den letzten Jahren nur ein einziger Patient behan- } \\
\text { delt. }\end{array}$ \\
\hline Luxemburg & $\begin{array}{l}2001: \\
\text { E-111: } 4.101 \text { Personen } \\
\text { E-112: } 250 \text { Personen }\end{array}$ \\
\hline Niederlande & Für das Jahr 2000: 3.316 Personen. \\
\hline Österreich & 2000: rund 5.160.000 $€$, dies entspricht 1.000 Personen. \\
\hline
\end{tabular}




\begin{tabular}{|c|c|}
\hline Portugal & $\begin{array}{l}\text { Diese Daten werden dezentral erfasst, auf nationaler Ebene liegen } \\
\text { keine Informationen vor. Die portugiesischen Behörden bemühen sich } \\
\text { um die Beschaffung dieser Informationen }\end{array}$ \\
\hline Finnland & $\begin{array}{l}\text { Für das Jahr } 2001 \\
\text { E-112: } 9 \text { Fälle, für die Finnland rund } 17.400 € \text { erstattet wurden. } \\
\text { E-111: es wurden } 11.483 \text { Vordrucke ausgestellt. } \\
\text { Insgesamt hat Finnland den ubrigen Mitgliedstaaten im Jahr } 2000 \\
\text { einen Betrag von } 877.000 € \text { und im Jahr } 2001 \text { einen Betrag von } \\
951.000 € \text { in Rechnung gestellt. }\end{array}$ \\
\hline Schweden & $\begin{array}{l}\text { Die schwedischen Behörden haben Informationen in Form von Fi- } \\
\text { nanzsummen übermittelt. } \\
\text { Für das Jahr 2000: } \\
\text { 33,5 Millionen SEK ( } \pm 3.666 .411 € \text { ), die für die Behandlung von } \\
\text { Staatsangehörigen anderer Mitgliedstaaten erstattet wurden } \\
53,1 \text { Millionen SEK ( } \pm 5.838 .000 € \text { ) wurden für die Behandlung von } \\
\text { Staatsangehörigen der übrigen skandinavischen Länder gezahlt. Die } \\
\text { Erstattung dieser Beträge wird bei den betreffenden Ländern nicht } \\
\text { beantragt. }\end{array}$ \\
\hline $\begin{array}{l}\text { Vereinigtes } \\
\text { Königreich }\end{array}$ & $\begin{array}{l}2001 \text { wurden } 871 \text { Patienten im Rahmen des Formblatts E-112 im Ver- } \\
\text { einigten Königreich behandelt, dies entspricht einem Betrag in Höhe } \\
\text { von 5,56 Millionen Pfund }( \pm 8.720 .428 €) \text {, darunter } 641 \text { Patienten aus } \\
\text { Irland und } 121 \text { Patienten aus Italien. } \\
2002 \text { beträgt diese Zahl 776, dies entspricht einem Betrag von } 1,21 \\
\text { Millionen Pfund ( } \pm 1897791 €) \text {, darunter } 659 \text { Patienten aus Irland und } \\
60 \text { aus Italien. }\end{array}$ \\
\hline
\end{tabular}

Quelle: Europäische Kommission (2003a), Anhang 2, S. 27ff, eigene Darstellung. 
Tabelle IV: Zahl der Personen, die eine Genehmigung einer Behandlung im Ausland beantragt haben

\begin{tabular}{|c|c|}
\hline Belgien & $\begin{array}{l}\text { Die belgischen Behörden haben Informationen in Form von Fi- } \\
\text { nanzsummen übermittelt. } \\
\text { Für das Jahr } 2000 \\
\text { Ausgaben der belgischen Versicherten im Ausland im Bereich ab- } \\
\text { hängig Beschäftigte: } 4.213 .474 .790 \mathrm{FB}(104.449 .312 €) \text {, seit } 1999 \\
\text { Rückgang um } 12,50 \% \text {. } \\
\text { Ausgaben der ausländischen Versicherten in Belgien im Bereich } \\
\text { Selbständige: } 113.149 .378 \mathrm{FB}(2.804 .890 €) \text {, seit } 1999 \text { Rückgang } \\
\text { um } 23,49 \% \text {. }\end{array}$ \\
\hline Dănemark & $\begin{array}{l}\text { Über die Zahl der eingereichten oder bewilligten Geneh- } \\
\text { migungsanträge liegen keine genauen Daten vor. Seit Inkrafttreten } \\
\text { der Rechtsverordnung Nr. } 536 \text { vom 15. Juni 2000, die maximale } \\
\text { Wartezeiten vorsieht, wurden allerdings zwischen dem } 1.07 .02 \text { und } \\
\text { dem } 30.09 .0250 \text { Personen in einem anderen Mitgliedstaat (Schwe- } \\
\text { den und Deutschland) behandelt. } \\
2000 \text { wurden } 70 \text { Patienten, die aufgrund ihres Gesundheits- } \\
\text { zustandes Spezialbehandlungen benötigten, im Ausland behandelt, } \\
\text { einschließlich in der EU. } 2001 \text { beläuft sich ihre Zahl auf } 75 \text {. } \\
2000 \text { unterzogen sich } 21 \text { Krebspatienten einer Strahlenbehandlung } \\
\text { in Deutschland. } 2001 \text { beläuft sich ihre Zahl auf } 29 \text {. }\end{array}$ \\
\hline Spanien & $\begin{array}{l}2001 \text { wurden } 651 \text { Formblätter E-112 und } 57468 \text { Formblätter E-111 } \\
\text { ausgestellt. }\end{array}$ \\
\hline Frankreich & $\begin{array}{l}\text { Für den Zeitraum } 1996 \text { bis } 1999 \text { haben } 1.240 \text { Personen eine Ge- } \\
\text { nehmigung einer Behandlung im Ausland beantragt, } 789 \text { Anträge } \\
\text { wurden bewilligt ( } 64 \% \text { ). }\end{array}$ \\
\hline Irland & $\begin{array}{l}2000 \text { und } 2001 \text { haben rund } 650 \text { Personen pro Jahr eine Genehmi- } \\
\text { gung einer Behandlung im Ausland beantragt, davon } 600 \text { im Rah- } \\
\text { men der Vordrucke laut Verordnung 1408/71/EWWG. Irland hat } \\
2000 \text { und } 2001 \text { ca. } 7 \text { Millionen } € \text { für Patienten erstattet, die in ande- } \\
\text { ren Mitgliedstaaten behandelt wurden. } \\
2002 \text { hat Irland den "National Treatment Purchase Fund" gegrun- } \\
\text { det, um die Wartelisten zu verkürzen; dazu wurden private Anbie- } \\
\text { ter in Irland und im Ausland herangezogen. Dieser Fonds hat } 2002 \\
6,6 \text { Millionen } € \text { für } 1.920 \text { Patienten ausgegeben, die entweder in } \\
\text { Privatkliniken in Irland oder im Vereinigten Königreich behandelt } \\
\text { wurden. }\end{array}$ \\
\hline
\end{tabular}




\begin{tabular}{|c|c|}
\hline Italien & $\begin{array}{l}1999 \text { haben } 21.300 \text { Personen eine Genehmigung einer Behandlung } \\
\text { im Ausland beantragt. } \\
\text { Die Zahl der Fälle, die sich auf die Vordrucke laut Verordnung } \\
\text { 1408/71/EWG stützen, beträgt 1999 16.280, dies entspricht } \\
49.952 .314 € \text { (unvollständige Daten). Die Beträge aufgrund der au- } \\
\text { Berhalb der Verordnung erteilten Genehmigungen entsprechen einer } \\
\text { Summe von 19.980.840€ (unvollständige Daten). }\end{array}$ \\
\hline Luxemburg & $\begin{array}{l}2001 \text { haben } 11.751 \text { Personen eine Genehmigung einer Behandlung } \\
\text { im Ausland beantragt, davon } 4.272 \text { Krankenhausbehandlungen da- } \\
\text { von wiederum } 74 \text { Kuren } \\
\text { Es wurden } 245 \text { Anträge abgelehnt. }\end{array}$ \\
\hline Österreich & $\begin{array}{l}\text { Zahl der Vordrucke E-112: 850, dies entspricht } 4.724 .000 €, \mathrm{~d} . \mathrm{h} \text {. } \\
\text { 0,0154\% aller Versicherten in Österreich. } \\
\text { Die Zahl der abgelehnten Anträge ist nicht bekannt. }\end{array}$ \\
\hline Portugal & $\begin{array}{l}\text { Aufgrund der zerstreuten Entscheidungszentren liegt für den Vor- } \\
\text { druck E-112 kein Zahlenmaterial vor. } \\
2001 \text { wurden } 260 \text { Genehmigungsanträge im Rahmen der Gesetzes- } \\
\text { verordnung Nr. } 177 / 92 \text { gestellt. } 246 \text { wurden bewilligt. }\end{array}$ \\
\hline Finnland & $\begin{array}{l}2001 \text { wurden } 9 \text { Genehmigungen im Rahmen des Formblatts E-112 } \\
\text { erteilt, dies entspricht } 17.900 € \text {. } \\
2002 \text { wurden } 4 \text { Genehmigungen im Rahmen des Vordrucks E-112 } \\
\text { erteilt. } 2001 \text { wurden } 24.143 \text { Vordrucke E-111 ausgestellt } \\
\text { Insgesamt hat Finnland } 2001 \text { den übrigen Mitgliedstaaten 1,7 Milli- } \\
\text { onen } € \text { erstattet. }\end{array}$ \\
\hline Schweden & $\begin{array}{l}\text { Die schwedischen Behörden erklären, dass bei ihnen nur wenige } \\
\text { Anträge auf Behandlung im Ausland eingehen. } \\
2002 \text { wurden } 6 \text { Anträge im Rahmen des Vordrucks E-112 gestellt, } \\
\text { die alle abgelehnt wurden. } \\
\text { Die schwedischen Behörden stellen jährlich rund } 400.000 \text { Vordru- } \\
\text { cke E-111 aus. }\end{array}$ \\
\hline
\end{tabular}




\begin{tabular}{|l|l|}
\hline Vereinigtes & $\begin{array}{l}2000 \text { haben 1.100 Personen eine Genehmigung einer Behandlung im } \\
\text { Ausland im Rahmen von E-112 beantragt. 2001 beziffert sich ihre } \\
\text { Zahl auf 1.134. } \\
\text { Auf zentraler Ebene liegen über die Verfahren außerhalb von E-112, } \\
\text { die von den kommunalen Behörden (Primary Care Trusts) bearbeitet } \\
\text { werden, keinerlei Daten vor. Das Vereinigte Königreich hat aller- } \\
\text { dings ein Projekt gestartet, bei dem Patienten, die auf eine Behand- } \\
\text { lung warten, eine Behandlung in einem anderen Mitgliedstaat vor- } \\
\text { geschlagen wird. Dieses Projekt wird von den Kommunalbehörden } \\
\text { (Primary Care Trusts) verwaltet. } \\
\text { In der Pilotphase wurden 190 Patienten behandelt, 269 seit Ab- } \\
\text { schluss der Pilotphase. Die Kosten der Pilotphase belaufen sich auf } \\
\text { 1,1 Millionen Pfund ( } 1.725 .265 €), \text { die Kosten der Phase im An- } \\
\text { schluss daran auf } 770.000 \text { Pfund }( \pm 1.207 .685 €)\end{array}$ \\
\hline
\end{tabular}

Quelle: Europäische Kommission (2003a), Anhang 2, S. 27ff, eigene Darstellung. 
Tabelle V: Prozentsatz der erteilten Genehmigungen (a) und Zahl der beantragten Erstattungen ohne Genehmigung (b)

\begin{tabular}{|l|l|}
\hline Belgien & $\begin{array}{l}\text { Ausgaben in Verbindung mit der Durchführung der Urteile Kohll und } \\
\text { Decker: } \\
\text { 1999: 3.521.039 FB (87.284 } € \text { ) } \\
\text { 2000: 5.423.627 FB (134.448 €) } \\
\text { 2001: 9.061.907 FB (224.639 €). }\end{array}$ \\
\hline Frankreich & $\begin{array}{l}\text { a) Für den Zeitraum 1996 bis 1999 wurden 64 \% aller beantragten Ge- } \\
\text { nehmigungen erteilt. } \\
\text { b) Von den lokalen Krankenkassen erhobene Daten - nicht verfügbar }\end{array}$ \\
\hline Irland & $\begin{array}{l}\text { a) über 90\% der Anträge wurden bewilligt. } \\
\text { b) Vor kurzem haben 14 Personen die Erstattung der in einem anderen } \\
\text { Mitgliedstaat angefallenen Krankheitskosten beantragt, ohne dass ih- } \\
\text { nen eine Genehmigung erteilt wurde. }\end{array}$ \\
\hline Italien & $\begin{array}{l}\text { a) 1999 wurden 91,5\% der beantragten Genehmigungen erteilt. } \\
\text { b) 1999 wurden 4 Erstattungsanträge ohne vorherige Genehmigung } \\
\text { gestellt. }\end{array}$ \\
\hline Luxemburg & $\begin{array}{l}\text { 2001 } \\
\text { a) Zahl der abgelehnten Anträge: } 245 \\
\text { Ablehnungsgründe: die Anträge wurden nachträglich gestellt, doch } \\
\text { wenn die Anträge Behandlungen außerhalb von Krankenhäusern } \\
\text { betreffen, gewähren die luxemburgischen Behörden eine Kostenerstat- } \\
\text { tung aufgrund der Urteile Kohll und Decker. } \\
\text { b) Zahl der Personen, die im Rahmen der Vordrucke einen Antrag ge- } \\
\text { stellt haben: } \\
\text { Vordruck E-112: 11.751 } \\
\text { Andere Vordrucke: 71.792, darunter Formblätter E-106: 67.658 } \\
\text { Beträge: gesamt 133.768.556 } € \text {, davon Vordrucke E-112: 24.513.299 } \\
€ \\
\text { davon Grenzgänger : 95.265.481 } €\end{array}$ \\
\hline Portugal & $\begin{array}{l}\text { Die Kosten für die Behandlung von 58.000 Personen im Ausland wer- } \\
\text { den ohne vorherige Genehmigung erstattet (1,0517\% der Versicherten } \\
\text { in Österreich), dies entspricht 3.445.470€. Die österreichischen Be- } \\
\text { hörden teilen mit, dass es sich dabei um Leistungen außerhalb von } \\
\text { Krankenhäusern handelt (hauptsächlich Kosten für Zahnbehandlun- } \\
\text { gen), die in Ungarn erbracht wurden. }\end{array}$ \\
b) 1 Antrag 2001. \\
\hline
\end{tabular}


a) 193 auf den Vordruck E-112 gestützte Anträge wurden 2000 abgeKönigreich lehnt, 253 im Jahr 2001.

b) Die Behörden sind zwar über eine Reihe von Fällen auf dem Laufenden, es liegen ihnen jedoch keine genauen Angaben vor.

a) Prozentsatz der erteilten Genehmigungen

b) Zahl der Personen, die eine Erstattung beantragt haben, ohne dass ihnen eine Genehmigung erteilt wurde.

Quelle: Europäische Kommission (2003a), Anhang 2, S. 27ff, eigene Darstellung. 


\section{Anhang C: Daten zur Finanzierung der Gesundheitsausgaben}

Tabelle VI: Anteil der verschiedenen Finanzierungsträger an den Gesundheitsausgaben, 2003

\begin{tabular}{|c|c|c|c|}
\hline \multirow[t]{2}{*}{ Land } & \multicolumn{2}{|c|}{$\begin{array}{l}\text { Anteil der öffentlichen Ausgaben an } \\
\text { allen Gesundheitsausgaben }\end{array}$} & \multirow{2}{*}{ Private Zahlungen } \\
\hline & $\begin{array}{l}\text { Sozialversiche- } \\
\text { rung }\end{array}$ & $\begin{array}{l}\text { Sonstige öffentliche } \\
\text { Finanzierungsträger }\end{array}$ & \\
\hline Belgien & 55,3 & 15,9 & 28,8 \\
\hline Dänemark & 0,0 & 82,9 & 17,1 \\
\hline Deutschland & 68,6 & 9,9 & 21,5 \\
\hline Estland & 65,6 & 10,7 & 23,7 \\
\hline Finnland & 15,9 & 59,8 & 24,3 \\
\hline Frankreich & 73,6 & 2,4 & 24,0 \\
\hline Griechenland & 18,8 & 34,1 & 47,1 \\
\hline Großbritannien & 0,0 & 83,4 & 16,6 \\
\hline Irland & 0,6 & 74,6 & 24,8 \\
\hline Island & 22,7 & 61,3 & 16,0 \\
\hline Italien & 0,1 & 75,5 & 24,4 \\
\hline Lettland & 31,8 & 32,3 & 35,9 \\
\hline Litauen & 62,6 & 10,0 & 27,4 \\
\hline Luxemburg & 80,3 & 5,1 & 14,6 \\
\hline Malta & 48,0 & 23,7 & 28,3 \\
\hline Monaco & 78,4 & 1,2 & 20,4 \\
\hline Niederlande & 61,5 & 4,1 & 34,4 \\
\hline Norwegen & 0,0 & 83,5 & 16,5 \\
\hline Österreich & 41,2 & 28,7 & 30,1 \\
\hline Polen & 63,3 & 9,1 & 27,6 \\
\hline Portugal & 4,6 & 65,9 & 29,3 \\
\hline Rumänien & 50,9 & 15,0 & 34,1 \\
\hline Schweden & 0,0 & 85,3 & 14,7 \\
\hline Schweiz & 40,0 & 17,9 & 42,1 \\
\hline Slowakei & 82,9 & 6,5 & 10,6 \\
\hline Slowenien & 65,2 & 9,7 & 25,1 \\
\hline Spanien & 5,1 & 66,2 & 28,7 \\
\hline Tschechien & 81,5 & 9,9 & 8,6 \\
\hline Türkei & 32,6 & 33,2 & 34,2 \\
\hline Ungarn & 57,1 & 13,1 & 29,8 \\
\hline Zypern & 0,0 & 41,3 & 58,7 \\
\hline
\end{tabular}

Quelle: World Health Organization (WHO) (2005). 


\section{Literaturverzeichnis}

Allgemeine Ortskrankenkasse (AOK) (2005a): Lexikon - Arzneimittelzulassung, www.aok-bv.de/lexikon/a/index_02155.html, (Abrufdatum: 15.11.2005).

Allgemeine Ortskrankenkasse (AOK) (2005b): Lexikon - Bedarfsplanung Ärzte, Zahnärzte, www.aok-bv.de/lexikon/b/index_02444.html, (Abrufdatum: 01.03.2005).

Allgemeine Ortskrankenkasse (AOK) (2005c): Lexikon - Bioverfügbarkeit, www.aok-bv.de/lexikon/b/index_02496.html, (Abrufdatum: 29.05.2005).

Allgemeine Ortskrankenkasse (AOK) (2005d): Lexikon - Heilmittelwerbegesetz, www.aok-bv.de/politik/gesetze/index_02996.html, (Abrufdatum: 14.07.2005).

Allgemeine Ortskrankenkasse (AOK) (2005e): Lexikon - Krankenhausfinanzierung, www.aok-bv.de/lexikon/k/index_02244.html, (Abrufdatum: 29.05.2005).

Allgemeine Ortskrankenkasse (AOK) (2005f): Lexikon - Public Health, www.aokbv.de/lexikon/p/index_02369.html, (Abrufdatum: 15.03.2005).

Allgemeine Ortskrankenkasse (AOK) (2005g): Lexikon - Verschreibungspflicht, www.aok-bv.de/lexikon/v/index_02567.html, (Abrufdatum: 12.05.2006).

Apothekerkammer Westfalen-Lippe (2004): Medikamenten-Versandhandel fristet ein Nischendasein, Pressemitteilung vom 05.11.04, Münster.

Arbeitsgemeinschaft der Spitzenverbănde der gesetzlichen Krankenkassen (2002): Die offene Methode der Koordinierung im Bereich des Gesundheitswesens, o.O.

Arbeitsgemeinschaft der Spitzenverbănde der gesetzlichen Krankenkassen (2004): Europa für die Versicherten gestalten: Aktuelle europäische Entwicklungen im Bereich der Gesundheitspolitik, 0.0 .

Arnold, M.; Armann, W. (1991): Zur Vergütung des Kassenarztes, in: Arbeit und Sozialpolitik, Bd. 45, Nr. 7-8, S. 29-32.

Association Européene des Spécialités Pharmaceutiques Grand Public (AESGP) (2004a): Economic and Legal Framework for Non-Prescription Medicines: Netherlands, Brüssel.

Association Européene des Spécialités Pharmaceutiques Grand Public (AESGP) (2004b): Economic and Legal Framework for Non-Prescription Medicines: United Kindom, Brüssel.

Becker, U. (2003): Gesetzliche Krankenversicherung im Europäischen Binnenmarkt, in: Neue Juristische Wochenschrift, Bd. 56, Nr. 32, S. 2272-2277. 
Beratungsgesellschaft für Angewandte Systemforschung (Basys); Infras (2002): Auswirkungen staatlicher Eingriffe auf das Preisniveau im Bereich Humanarzneimittel, Bern.

Berg, W. (1997): Gesundheitsschutz als Aufgabe der EU: Entwicklung, Kompetenzen, Perspektiven, Baden-Baden.

Berufsordnung für Apotheker des Landes Niedersachsen (1993) vom 19. November 1993 in der Fassung vom 19. November 1997.

Bieback, K.J. (2001): Etablierung eines Gemeinsamen Marktes für Krankenbehandlungen durch den EuGH - Das Urteil des EuGH „Smits/Peerbooms“ vom 12.7.2001 -, in: Neue Zeitschrift für Sozialrecht, Bd. 10, Nr. 11, S. 561-569.

BlöB, T. (2006): DocMorris prescht vor, in: Deutsches Ärzteblatt, Bd. 103, Nr. 33, S. A 2146.

Boroch, W. (1994): Internationale Wettbewerbsfähigkeit der EU-Arzneimittelindustrie: Markttheoretische Grundlagen, empirische Ergebnisse und binnenmarktpolitische Handlungserfordernisse, Hamburg.

Breyer, F.; Zweifel, P.; Kifmann, M. (2005): Gesundheitsökonomie, Berlin [u.a.].

Brümmerhoff, D. (2001): Finanzwissenschaft, München [u.a.].

Bundesagentur für Arbeit (BfA) / Zentralstelle für Arbeitsvermittlung (ZAV) (2004a): Der Arbeitsmarkt für hoch qualifizierte Fach- und Führungskräfte, in: Informationen für die Beratungs- und Vermittlungsdienste (ibv), Nr. 11.

Bundesagentur für Arbeit (BfA) / Zentralstelle für Arbeitsvermittlung (ZAV) (2004b): Ärztinnen und Ärzte - Facharztmangel droht, Arbeitsmarktinformation für qualifizierte Fach- und Führungskräfte, Bonn.

Bundesministerium für Gesundheit (BMG) (2005): Statistisches Taschenbuch Gesundheit 2005, Berlin.

Bundesministerium für Gesundheit (BMG) (2006a): GKV Statistik BMG, Vordruck KV 45, Berlin.

Bundesministerium für Gesundheit (BMG) (2006b): Kennzahlen und Faustformeln GKV, Berlin.

Bundesministerium für Gesundheit und Soziale Sicherheit (BMGS) (2005a): Ambulante und stationäre medizinische Vorsorge- und Rehabilitationsleistungen (Kuren), www.bmgs.bund.de/deu/gra/themen/gesundheit/gesetzl/index_3442.php, (Abrufdatum: 23.09.2006). 
Bundesministerium für Gesundheit und Soziale Sicherung (BMGS) (2005b): Den Niederlanden und dem Vereinigten Königreich ist der Versandhandel mit Arzneimitteln nach Deutschland erlaubt, Pressemitteilung vom 21.06.2005, Berlin.

Bundesministerium für Gesundheit und Soziale Sicherung (BMGS) (2005c): Europa, www.bmgs.bund.de/deu/gra/themen/europa/, (Abrufdatum: 31.01.2005).

Bundesministerium für Gesundheit und Soziale Sicherung (BMGS) (2005d): Wie wurden die neuen Regelungen gestaltet, www.bmg.bund.de/cln_041/nn_599776/ sid_DD50C0553EB1E2733C4F0F205922B4A4/DE/Themenschwerpunkte/Gesundhei $\mathrm{t} /$ Arzneimittel/Wie-wurden-die-neuen-Regelung-5390,param $=. \mathrm{html}$ nnn=true, $\quad(\mathrm{Ab}$ rufdatum: 16.06 .2005 ).

Bundesverband der Freien Berufe (BFB) (2006): Definition, www.freie-berufe.de/ Definition.212.0.html, (Abrufdatum: 12.09.2006).

Bundesverband der pharmazeutischen Industrie (BPI) (2004): Pharma-Daten 2004, Berlin.

Bundesverband der Pharmazeutischen Industrie (BPI) (2006): Pharma-Daten 2006, Berlin.

Bundesverband Deutscher Postdienstleister (BvDP) (2005): Postbank Studie: Medikamentenhandel im Internet nimmt $2005 \mathrm{zu}$, www.bvdp.de/index.htm?/files/briefekep/4C6E66F7D7E149DDA5E964D49DEF3BB7.htm, (Abrufdatum: 15.6.2005).

Bundesvereinigung Deutscher Apothekerverbănde (ABDA) (2002): Jahresbericht 2001/2002, Eschborn.

Bundesvereinigung Deutscher Apothekerverbände (ABDA) (2004): Jahresbericht 2003/2004, Eschborn.

Bundesvereinigung Deutscher Apothekerverbände (ABDA) (2005): Jahresbericht 2004/2005, Eschborn.

Bundesvereinigung Deutscher Apothekerverbände (ABDA) (2006): Jahresbericht 2005/2006, Eschborn.

Bundesverfassungsgericht (Hrsg.) (1958): Entscheidungen des Bundesverfassungsgerichts, 7. Band, Tübingen.

Clade, H. (1997): Lichtblick im Reha-Bereich, in: Deutsches Ärzteblatt, Bd. 94, Nr. $34 / 35$, S. A 2178.

Cullis, J.G.; West, P.A. (1979): The Economics of Health: An Introduction, Oxford.

Danzon, P.M. (1997a): Pharmaceutical Price Regulation: National Policies versus Global Interests, Washington D.C. 
Danzon, P.M. (1997b): Price Discrimination for Pharmaceuticals: Welfare Effects in the US and the EU, in: International Journal of the Economics of Business, Bd. 4, Nr. 3, S. 301-321.

Danzon, P.M. (1998): The Economics of Parallel Trade, in: Pharmacoeconomics, Bd. 13, Nr. 3, S. 293-304.

Danzon, P.M.; Chao, L. (2000): Does Regulation drive out Competition in Pharmaceutical Markets? in: The Journal of Law und Economics, Bd. 43, Nr. 2, S. 311-357.

Danzon, P.M.; Furukawa, M.F. (2003): Prices and Availibility of Pharmaceuticals: Evidence from Nine Countries, in: Health Affairs - Web Exclusive, Oktober, S. 521536.

Das Erste - Plusminus (2005): Kur im Ausland. Der plusminus-Tipp! www.daserste. de/plusminus/beitrag_archiv.asp?aid=330, (Abrufdatum: 26.09.2006).

Department of Health (2005): Generic medicines, www.dh.gov.uk/PolicyAndGuid ance/MedicinesPharmacyAndIndustry/GenericMedicines/fs/en, (Abrufdatum: 30.07. 2005).

Deutscher Bundesrat (2004): Beschluss des Bundesrates, in: Bundesratsdrucksache Nr. 365/04 (Beschluss) vom 11.06.04.

Deutscher Heilbäderverband (DHV) (2005): Heilbäder und Kurorte in der zukünftigen Gesundheitspolitik, www.deutscher-heilbaederverband.de/DB_Bilder/aktuelles /pdf/80.pdf, (Abrufdatum: 24.09.2005).

Deutscher Tourismusverband (2006): Tourismus in Deutschland 2005, Bonn.

DocMorris (2006): So sparen gesetzlich Versicherte, https://www.docmorris.de/de /vorteile/sparen_gkv/index.jsp;jsessionid=908B098F19175E67A8BC9B8FA2872133. eul246, (Abrufdatum: 15.6.2006).

Domscheit, A. (1998): Freier EG-Binnenmarkt rüttelt an den nationalen Sozialversicherungssystemen, in: Die Krankenversicherung, Bd. 50, Nr. 8/9, S. 246-250.

Eichenhofer, E. (1999): Das Europäische koordinierende Krankenversicherungsrecht nach den EuGH-Urteilen Kohll und Decker, in: Vierteljahresschrift für Sozialrecht, Nr. 2, S. 101-122.

Eichenhofer, E. (2001): Richtlinien der gesetzlichen Krankenversicherung und Gemeinschaftsrecht, in: Neue Zeitschrift für Sozialrecht, Bd. 10, Nr. 1, S. 1-7.

Europa Apotheek Venlo (2006): Preisvorteile - für Sie und Ihre Krankenkasse, www.europa-apotheek.com/popup_bonus.php, (Abrufdatum: 20.08.2006). 
Europăische Kommission (1991): Preliminary Draft: Measures Amining at Modifying and Completing Directive 89/105/EEC (III/3001-EN) Concerning the Pricing and Reimbursement of Medicinal Products for Human Use / rev. 2, Brüssel.

Europäische Kommission (1993): Mitteilung über den Aktionsrahmen im Bereich der öffentlichen Gesundheit, KOM (93) 559 endg., Luxemburg.

Europäische Kommission (1997): Förderung der Innovation durch Patente: Grünbuch über das Gemeinschaftspatent und das Patentschutzsystem in Europa, KOM (1997) 314 endg., Luxemburg.

Europäische Kommission (1998): Mitteilung über die Entwicklung der Gemeinschaftspolitik im Bereich der öffentlichen Gesundheit, KOM (1998) 230 endg., Luxemburg.

Europäische Kommission (1999): Förderung der Innovation durch Patente: Folgemaßnahmen zum Grünbuch über das Gemeinschaftspatent und das Patentschutzsystem in Europa, KOM (1999) 42 endg., Luxemburg.

Europăische Kommission (2000a): Mitteilung über die gesundheitspolitische Strategie der Europäischen Gemeinschaft, KOM (2000) 285 endg., Luxemburg.

Europăische Kommission (2000b): Pharmaceuticals in the European Union, Luxemburg.

Europäische Kommission (2000c): Vorschlag für eine Verordnung des Rates über das Gemeinschaftspatent, KOM (2000) 412 endg., Luxemburg.

Europäische Kommission (2001): Die Zukunft des Gesundheitswesens und der Altenpflege: Zugänglichkeit, Qualität und langfristige Finanzierbarkeit sichern, KOM (2001) 723 endg., Luxemburg.

Europåische Kommission (2002): Mitteilung: Freizügigkeit der Arbeitnehmer - Volle Nutzung der Vorteile und Möglichkeiten, KOM (2002) 694 endg., Luxemburg.

Europăische Kommission (2003a): Arbeitsdokument der Kommissionsdienststellen. Zusammenfassender Bericht. Anwendung der Binnenmarktvorschriften im Bereich der Gesundheitsdienste. Durchführung der Rechtsprechung des Gerichtshofes durch die Mitgliedstaaten, SEK (2003) 900, Brüssel.

Europäische Kommission (2003b): Die pharmazeutische Industrie Europas zum Wohl der Patienten stärken: Was zu tun ist, KOM (2003) 383 endg., Luxemburg.

Europäische Kommission (2003c): Mitteilung hinsichtlich der Überprüfung der Richtlinie 93/104/EG über bestimmte Aspekte der Arbeitszeitgestaltung, KOM (2004) 843 endg., Luxemburg. 
Europäische Kommission (2003d): Mitteilung über die Stärkung der sozialen Dimension der Lissabonner Strategie: Straffung der offenen Koordinierung im Bereich Sozialschutz, KOM (2003) 261 endg./2, Luxemburg.

Europăische Kommission (2003e): Mitteilung über Paralleleinfuhren von Arzneispezialităten, deren Inverkehrbringen bereits genehmigt ist, KOM (2003) 839 endg., Luxemburg.

Europăische Kommission (2003f): Reflexionsprozess auf hoher Ebene über die Patientenmobilität und die Entwicklung der gesundheitlichen Versorgung in der Europäischen Union, Brüssel.

Europäische Kommission (2004a): Geänderter Vorschlag für eine Richtlinie des Europäischen Parlaments und des Rates zur Änderung der Richtlinie 2003/88/EG über bestimmte Aspekte der Arbeitszeitgestaltung, KOM (2004) 246 endg., Luxemburg.

Europäische Kommission (2004b): Modernisierung des Sozialschutzes für die Entwicklung einer hochwertigen, zugänglichen und zukunftsfähigen Gesundheitsversorgung und Langzeitpflege: Unterstützung der einzelstaatlichen Strategien durch die „offene Koordinierungsmethode“, KOM (2004) 304 e, Luxemburg.

Europäische Kommission (2004c): Vorschlag für eine Richtlinie des Europäischen Parlament und Rates über Dienstleistungen im Binnenmarkt, KOM (2004) 2 endg./2, Brüssel.

Europäische Kommission (2004d): Vorschlag für eine Richtlinie des Europäischen Parlaments und des Rates zur Änderung der Richtlinie 2003/88/EG über bestimmte Aspekte der Arbeitszeitgestaltung, KOM (2004) 607 endg., Luxemburg.

Europåische Kommission (2005a): Gemeinsame Maßnahmen für Wachstum und Beschäftigung: Das Lissabon-Programm der Gemeinschaft, KOM (2005) 330 endg., Luxemburg.

Europäische Kommission (2005b): Gemeinsamer Bericht über Sozialschutz und soziale Eingliederung, KOM (2005) 14 endg., Luxemburg.

Europäische Kommission (2005c): Koordinierung der Sozialversicherungssysteme, europa.eu.int/comm/employment_social/social_security_schemes/index_de.htm, (Abrufdatum: 24.2.2005).

Europåische Kommission (2005d): Mehr Gesundheit, Sicherheit und Zuversicht für die Bürger - Eine Gesundheits- und Verbraucherschutzstrategie, KOM (2005) 115 endg., Luxemburg.

Europäische Kommission (2005e): Mitteilung: Zusammenarbeiten, zusammen mehr erreichen: ein neuer Rahmen für die offene Koordinierung der Sozialschutzpolitik und der Eingliederungspolitik in der Europäischen Union, KOM (2005) 706, Brüssel. 
Europäische Kommission (2006a): Gemeinsamer Bericht über Sozialschutz und soziale Eingliederung 2006, KOM (2006) 62 endg., Brüssel.

Europăische Kommission (2006b): Patientenmobilität: Kommission leitet öffentliche Anhörung zum EU-Rahmen für die gesundheitliche Versorgung ein, Pressemitteilung IP/06/1150, Brüssel.

Europäische Kommission (2006c): Portfolio of Overarching Indicators and Streamlined Social Inclusion, Pension, and Health Portfolios, Brüssel.

Europåische Kommission (2006d): Vertragsverletzungsverfahren gegen Italien, Österreich und Spanen - Vorschriften für Apotheken unvereinbar mit dem Binnenmarkt, Pressemittelung IP/06/858, Brüssel.

Europăische Union (2004): Zusammenfassung der Gesetzgebung: Gemeinschaftspatent, europa.eu.int/scadplus/leg/de/lvb/126056.htm, (Abrufdatum: 14.08.2005).

Europäischer Gerichtshof (1974a): Urteil in der Rechtsache 33/74 „van Binsbergen“ vom 03.12.1974.

Europäischer Gerichtshof (1974b): Urteil in der Rechtssache 2/74 „Reyners“ vom 21.06.1974.

Europåischer Gerichtshof (1974c): Urteil in der Rechtssache 8/74 „Dassonville“ vom 11.07.1974.

Europäischer Gerichtshof (1976): Urteil in der Rechtsache 104/75 „de Peijper“ vom 20.05.1976.

Europåischer Gerichtshof (1979): Urteil in der Rechtssache 120/78 „Rewe / Bundesmonopolverwaltung für Brandwein" vom 20.02.1979.

Europäischer Gerichtshof (1981): Urteil in der Rechtsache 246/80 „Broekmeulen“ vom 06.10.1981.

Europåischer Gerichtshof (1991): Urteil in der Rechtsache C-41/90 „Höfner und Elser" vom 23.04.1991.

Europäischer Gerichtshof (1993a): Urteil in der Rechtsache C-19/92 „Krauß“ vom 31.03.1993.

Europäischer Gerichtshof (1993b): Urteil in der Rechtsache C-159/91 „Poucet und Pistre" vom 17.02.1993.

Europäischer Gerichtshof (1995): Urteil in der Rechtsache C-55/94 „Gebhard“ vom 30.11.1995. 
Europäischer Gerichtshof (1996): Urteil in der Rechtssache C-201/94 „The Queen / The Medicines Control Agency, ex parte: Smith \& Nephew Pharmaceuticals und Primecrown / Medicins Control Agency“ vom 12.11.1996.

Europalischer Gerichtshof (1997): Urteil in der Rechtsache C-159/94 „Kommission / Frankreich" vom 23.10.1997.

Europåischer Gerichtshof (1998a): Urteil in der Rechtsache C-35/96 „Kommission / Italien" vom 18.06.1998.

Europäischer Gerichtshof (1998b): Urteil in der Rechtssache C-120/95 „Decker“ vom 28.04.1998.

Europăischer Gerichtshof (1998c): Urteil in der Rechtssache C-158/96 „Kohll“ vom 28.04.1998.

Europäischer Gerichtshof (1998d): Urteil in der Rechtssache C-368/96 „Generics (UK) u.a." vom 03.12.1998.

Europåischer Gerichtshof (1999): Urteil in der Rechtsache C-67/96 „Albany“ vom 21.09.1999.

Europăischer Gerichtshof (2001a): Urteil in der Rechtssache C-157/99 „Smits und Peerbooms" vom 12.07.2001.

Europaischer Gerichtshof (2001b): Urteil in der Rechtssache C-368/98 „Vanbraekel" vom 12.07.2001.

Europäischer Gerichtshof (2002): Urteil in der Rechtsache C-218/00 „Cisal“" vom 22.01.2002.

Europäischer Gerichtshof (2003a): Schlussanträge der Generalstaatsanwältin zur Rechtsache C-322/01 „Deutscher Apothekerverband e. V. gegen 0800 DocMorris NV und Jacques Waterval" vom 11.03.2003.

Europåischer Gerichtshof (2003b): Schlussantrăge des Generalanwalts zu den verbundenen Rechtssachen C-264/01, C-306/01, C-354/01 und C-355/01 „AOKBundesverband u.a." vom 22.05.2003.

Europäischer Gerichtshof (2003c): Urteil in der Rechtssache C-151/02 „Jaeger“ vom 09.09.2003.

Europäischer Gerichtshof (2003d): Urteil in der Rechtssache C-322/01 „Deutscher Apothekerverband e. V. gegen 0800 DocMorris NV und Jacques Waterval" vom 11.12.2003.

Europäischer Gerichtshof (2003e): Urteil in der Rechtssache C-385/99 „MüllerFauré und van Riet" vom 13.05.2003. 
Europåischer Gerichtshof (2003f): Urteil in der Rechtssache C-56/01 „Inizan“ vom 23.10.2003.

Europäischer Gerichtshof (2004a): Schlussantrag des Generalstaatsanwalts zur Rechtsache C-53/03 „Syfait und andere“ vom 28.10.04.

Europäischer Gerichtshof (2004b): Urteil in den verbundenen Rechtssachen C264/01, C-306/01, C-354/01, C-355/01, „AOK-Bundesverband und andere“ vom 16.03.2004.

Europåischer Gerichtshof (2004c): Urteil in der Rechtssache C-8/02 „Leichtle“ vom 18.03.2004, Leichtle.

Europäischer Gerichtshof (2005): Urteil in der Rechtssache C-74/03 „SmithKline Beecham" vom 20.01.2005.

Europäischer Gerichtshof (2006): Urteil in der Rechtssache C-372/04 „Watts“ vom 16.05.2006.

Europäischer Rat (2000): Schlussfolgerungen des Vorsitzes, Europäischer Rat (Lissabon), 23. und 24 März 2000.

Europäischer Rat (2001): Schlussfolgerungen des Vorsitzes, Europäischer Rat (Göteborg), 15. und 16. Juni 2001.

Europäisches Gericht erster Instanz (EuG) (2000): Urteil in der Rechtssache T$41 / 96$ „Bayer / Kommission“ vom 20.10.2000.

Europäisches Gericht erster Instanz (EuG) (2003): Urteil in der Rechtsache T319/99 „Fenin / Kommission“ vom 04.03.2003.

Europåisches Parlament und Rat (2002): Beschluss Nr. 1786/2002/EG des europäischen Parlaments und des Rates vom 23. September 2002 über ein Aktionsprogramm der Gemeinschaft im Bereich der öffentlichen Gesundheit (2003-2008), in: ABl. L 271 vom 9.10.2002, S. 1-11.

European Federation of Pharmaceutical Industrie and Associations (EFPIA) (2000): The Pharmaceutical Industrie in Figures, Brissel.

Fiehn, A.; Schinnenburg, H. (2004): Krankenhäuser - Herausforderung Ärztemangel, in: Deutsches Ärzteblatt, Bd. 101, Nr. 10, S. A 626-A 628.

Flintrop, J. (2000): Kopfpauschalen, in: Deutsches Ärzteblatt, Bd. 97, Nr. 45, S. A 2976.

Friedrich, T.A. (2002): Europäisches Parlament erstreitet mehr Mittel für Gesundheit, in: Ärzte Zeitung online vom 05.07.2002, www.aerztezeitung.de/docs/2002/07/05 /124a0601.asp?cat=, (Abrufdatum: 08.12.2004). 
Fuchs, M. (2004): Das neue Recht der Auslandskrankenbehandlung, in: Neue Zeitschrift für Sozialrecht, Bd. 13, Nr. 5, S. 225-230.

Fuchs, M. (Hrsg.)(2005): Europäisches Sozialrecht: Nomos-Kommentar, BadenBaden.

Gemeinsamer Bundesausschluss (2006): Richtlinien des Bundesausschusses der Ärzte und Krankenkassen über die Bedarfsplanung sowie die Maßstäbe zur Feststellung von Überversorgung und Unterversorgung in der vertragsärztlichen Versorgung (Bedarfsplanungs-Richtlinien-Ärzte) vom 9. Mărz 1993 (Bundesanzeiger Nr. 110 a vom 18. Juni 1993), zuletzt geändert am 21. Februar 2006 veröffentlicht im Bundesanzeiger 2006 S. 2541, in Kraft getreten am 7. April 2006.

Gerlinger, T. (1997): Wettbewerbsordnung und Honorarpolitik, Frankfurt a. Main.

Gesellschaft für Versicherungswissenschaft und -gestaltung (GVG) (2002): Die GVG zur offenen Methode der Koordinierung im Gesundheitswesen, Informationsdienst der GVG Nr. 286, Köln.

Gesellschaft für Versicherungswissenschaft und -gestaltung (GVG) (2003): Die GVG zur Straffung der offenen Methode der Koordinierung im Bereich Sozialschutz, Informationsdienst der GVG Nr. 293, Köln.

Gesellschaft für Versicherungswissenschaft und -gestaltung (GVG) (2004): Die GVG zur „Offene Methode der Koordinierung“ im Bereich Gesundheitswesen und Langzeitpflege, Informationsdienst der GVG Nr. 300, Köln.

gesundheit.de (2005): Kuren im Ausland - Gleiche Qualität und gleiche Leistung? www.gesundheit.de/fitness-wellness/sanfte-medizin/kuren-im-ausland/index.html, (Abrufdatum: 20.09.2006).

Glaeske, G.; Klauber, J.; Lankers, C.H.; Selke, G.W. (2003): Stärkung des Wettwerbs in der Arzneimittelversorgung zur Steigerung von Konsumentennutzen, Effizienz und Qualität. Gutachten im Auftrag des Bundesministeriums für Gesundheit und Soziale Sicherung, Bonn.

Greß, S.; Axer, P.; Wasem, J. (2003): Europäisierung des Gesundheitswesens, Gütersloh.

Hackenbroch, V. (2003): Krankenhäuser: Einzug der Stechuhr, in: Der Spiegel, 15.09.2003, S. 166 .

Hancher, L. (2004): The European Community Dimension: Coordination Divergence, in: Mossialos, E.; Mrazek, M.; Walley, T. (Hrsg.): Regulating Pharmaceuticals in Europe: Striving for Efficiency, Equity and Quality, Maidenhead, S. 55-79. 
Hanika, H. (1998): Europäische Gesundheitspolitik: Stärkung der Kompetenzen der Europäischen Union im Gesundheitswesen durch die Neufassung des Artikels 152 EGVertrag, in: Medizinrecht, Bd. 16, Nr. 5, S. 193-196.

Hänlein, A. (1993): Zur Vereinbarkeit der Zulassungsbechränkungen für Vertragsärzte gemäß §§ 101 bis 1043 SGB V in der Fassung des Gesundheitsstrukturgesetzes mit höherrangigem Recht, in: Vierteljahresschrift für Sozialrecht, Nr. 3, S. 169-193.

Hănlein, A.; Kruse, J. (2000): Einflüsse des europäischen Wettbewerbsrechts auf die Leistungserbringung in der gesetzlichen Krankenversicherung, in: Neue Juristische Wochenschrift, Bd. 9, Nr. 4, S. 174.

Hanseatische Krankenkasse (HEK) (2005): Pro und Contra zu Kuraufenthalten im Ausland, www.hek.de/lookup.php?content=http://www.hek.de/content/6_4_2_5_1.htm, (Abrufdatum: 26.09.2006).

Hayek, F.A.v. (1969): Wettbewerb als Entdeckungsverfahren, in: Hayek, F.A.v. (Hrsg.): Freiburger Studien: Gesammelte Aufsätze, Tübingen, S. 249-265.

Heidenreich, M.; Bischoff, G. (2004): Forschungsprojekt: Die offene Methode der Koordinierung. Ein Weg zur Europäisierung der Sozial- und Beschäftigungspolitik, www.uni-bamberg.de/sowi/europastudien/dokumente/heidenreich_bischoff_2004_om k.pdf, (Abrufdatum: 02.02.2005).

Heidenreich, M.; Bischoff, G. (2006): The Open Method of Coordination. A way to the Europeanization of social and employment policies? in: Journal of Common Market Studies, in Druck.

High Level Group on Innovation and Provision of Medicines (2002): Recommendations for Action, Brüssel.

Hollmann, S.; Schulz-Weiden, W. (1998): Der Einfluß der EG auf das Gesundheitswesen der Mitgliedstaaten, in: Zeitschrift für ausländisches und internationales Arbeits- und Sozialrecht, Bd. 12, Nr. 2, S. 180-214.

Hollstein, P. (2006): Kettenkonzerne drängen in den Apothekenmarkt, in: Pharmazeutische Zeitung online, Nr. 26, www.pharmazeutische-zeitung.de/index.php?id=1476\& type $=1$, (Abrufdatum: 05.12.2006).

Hoppmann, E. (1967): Wettbewerb als Norm der Wettbewerbspolitik, in: Ordo: Jahrbuch für die Ordnung von Wirtschaft und Gesellschaft, Bd. 18, S. 77-94.

IKK Bundesverband (2005): Evaluation der Inanspruchnahme des Versandhandels durch IKK Versicherte bei Sanicare, Bad Laer, o.O. 
Jacobs, K. (2002): Vergütungssysteme und Wettbewerb in der Gesetzlichen Krankenversicherung, in: Wille, E. (Hrsg.): Anreizkompatible Vergütungssysteme im Gesundheitswesen, Baden-Baden, S. 11-33.

Jacobs, K.; Wasem, J. (2003): Weiterentwicklung einer leistungsfähigen und solidarischen Krankenversicherung unter den Rahmenbedingungen der europäischen Integration, Düsseldorf.

Jäger-Lindemann, S. (2004): Die Vereinbarkeit der Leistungserbringung in der gesetzlichen Krankenversicherung mit den Gemeinschaftsgrundfreiheiten, Berlin.

Jasper, J.; Tostmann, R. (2004): Ökonomische Auswirkungen von Strukturreformen im deutschen Apothekenmarkt, in: List-Forum für Wirtschafts- und Finanzpolitik, Bd. 30, Nr. 1, S. S. $20-40$.

Jorens, Y.; Coucheir, M.; Overmeiren, F.v. (2005): Zugang zu Gesundheitsleistungen im gemeinsamen Markt: Auswirkungen auf den gesetzlichen und den freiwilligen Gesundheitsschutz, Gent.

Juszczakm, J.; Zangerle, N. (2004): Patienten aus dem Ausland: Attraktives Geschäftsfeld für Krankenhäuser, in: Deutsches Ärzteblatt, Bd. 101, Nr. 17, S. A 1148-A 1150.

Kanavos, P.; Costa-Font, J. (2005): Pharmaceutical Parallel Trade in Europe: Stakeholder and Competition Effects, in: Economic Policy, Bd. 20, Nr. 44, S. 752-798.

Karlovy (Karlsbad) (2006): Statistik der Kurgäste in Karlsbad, www.karlovyvary.cz /data/prezentace/file_691_7.pdf, (Abrufdatum: 29.8.2005).

Kassenărztliche Bundesvereinigung (KBV) (1998): Grunddaten zur vertragsärztlichen Versorgung, daris.kbv.de/daris/link.asp?ID=1003730955, Abrufdatum 10.03. 2005).

Kassenärztliche Bundesvereinigung (KBV) (2005): Grunddaten zur vertragsärztlichen Versorgung, www.kbv.de/publikationen/2394.html, (Abrufdatum: 10.03.2005).

Kassenärztliche Bundesvereinigung (KBV) (2006): Grunddaten zur vertragsärztlichen Versorgung in Deutschland - Gesetzliche Krankenversicherung, www.kbv.de /publikationen/2422.html\#pkcvxdxijg, (Abrufdatum: 28.05.2007).

Kassenärztliche Bundesvereinigung (KBV) (2007): Grunddaten zur vertragsärztlichen Versorgung in Deutschland, www.kbv.de/publikationen/2394.html, (Abrufdatum: 23.02.2007).

Kingreen, T. (2001): Zur Inanspruchnahme von Gesundheitsleistungen im europäischen Binnenmarkt, in: Neue Juristische Wochenschrift, Bd. 54, Nr. 46, S. 3382-3385. 
Kingreen, T. (2006): Die grenzüberschreitende Inanspruchnahme und Erbringung von medizinischen Rehabilitationsleistungen, in: Zeitschrift für europäisches Sozial- und Arbeitsrecht, Bd. 5, Nr. 5/6, S. 210 - 215.

Klose, J.; Uhlemann, T.; Gutschmidt, S. (2003): Ärztemangel - Ärzteschwemme?: Auswirkungen der Altersstruktur von Ärzten auf die vertragsärztliche Versorgung, Bonn.

Koenig, C.; Engelmann, C. (2004): Das Festbetragsurteil des EuGH: Endlich Klarheit über den gemeinschaftsrechtlichen Unternehmensbegriff im Bereich der Sozialversicherung? in: Europäische Zeitschrift für Wirtschaftsrecht, Bd. 15, Nr. 22, S. 682686.

Koenig, C.; Engelmann, C.; Hentschel, K. (2003): Die Anwendbarkeit des Vergaberechts auf die Leistungserbringung im Gesundheitswesen, in: Medizinrecht, Bd. 21, Nr. 10, S. 562-568.

Koenig, C.; Engelmann, C.; Sander, C. (2001): Parallelhandelsbeschränkungen im Arzneimittelbereich und die Freiheit des Warenverkehrs, in: Gewerblicher Rechtsschutz und Urheberecht Internationaler Teil, Bd. 50, Nr. 11, S. 919-926.

Königbauer, I. (2004): Die Auswirkung von Parallelimporten auf die optimale Regulierung von Arzneimittelpreisen, in: Vierteljahrshefte zur Wirtschaftsforschung, Bd. 73, Nr. 4, S. 592-604.

Kopetsch, T. (2004): Die ärztliche Profession in Deutschland, in: Journal of Public Health, Bd. 12, Nr. 5, S. 304-320.

Kopetsch, T. (2006): Kassenärztliche Bedarfsplanung. Bessere Aussichten für Frauen- und Nervenärzte, in: Deutsches Ärzteblatt (PP), Bd. 5, Nr. 9, S. 397-399.

Kötter, U.E. (1999): Die Urteile des Gerichtshofs der Europäischen Gemeinschaften in den Rechtssachen Decker und Kohll: Der Vorhang zu und alle Fragen offen? in: Vierteljahresschrift für Sozialrecht, Nr. 4, S. 233-252.

Kötter, U.E. (2003): Die Entscheidung des EuGH in den Rechtsachen MüllerFauré/van Riet - Harmonisierung der sozialen Sicherung im Krankheitsfall durch die Rechtsprechung des EuGH? in: Zeitschrift für europäisches Sozial- und Arbeitsrecht, Bd. 2, Nr. 8, S. 301-311.

Kötter, U.E. (2003): Die Entscheidung des EuGH in den Rechtsachen MüllerFauré/van Riet - Harmonisierung der sozialen Sicherung im Krankheitsfall durch die Rechtsprechung des EuGH? in: Zeitschrift für europäisches Sozial- und Arbeitsrecht, Bd. 2, Nr. 8, S. 301-311.

Kotzian, P. (2003): Verhandlungen im europäischen Arzneimittelsektor, BadenBaden. 
Kröck, A.P. (1998): Der Einfluß der europäischen Grundfreiheiten am Beispiel der Ärzte und Arzneimittel: Über die Auswirkungen des Europarechts an der Schnittstelle zwischen mitgliedstaatlichen Kompetenzen im Sozialrecht und den Anforderungen des europäischen Binnenmarktes, Konstanz.

Lahm, C. (2005): Versandapotheke: Kernzielgruppe Senioren, in: Pharmazeutische Zeitung online, Nr. 09, www.pharmazeutische-zeitung.de/fileadmin/pza/200509/pol2.htm , (Abrufdatum 16.07.2006).

Laschet, H. (2003): Arznei-Importe - Bonanza für Händler und Apotheker, in Ärzte Zeitung online vom 09.10.2003, www.aerztezeitung.de/docs/2003/10/09/181a0601 .asp?cat=/politik, (Abrufdatum: 05.05.2006).

Mehnert, A. (1998): Regulierung auf europäischen Arzneimittelmärkten, Bern [u.a.].

Mehnert, A.; Wille, E.; Rohweder, J.P. (1994): Zum gesellschaftlichen Nutzen pharmazeutischer Innovationen, Frankfurt am Main [u.a.].

Merten, M. (2004a): Bedrückende Resignation, iil: Deutsches Ärzteblatt, Bd. 101, Nr. 47, S. A 3150-A 3152.

Merten, M. (2004b): Patientenmobilität in Europa: Von Tourismus keine Spur, in: Deutsches Ärzteblatt, Bd. 101, Nr. 27, S. A 1942-A 1944.

Meyer, H.J. (2005): Blick nach Brüssel, in: Arzneimittel und Recht, Bd. 1, Nr. 1, S. 18-19.

Münnich, F.E. (1998): Sind Sachleistungsprinzip, Budgetierung und Angebotssteuerung im europäischen Wettbewerb noch sinnvoll? in: Recht und Politik im Gesundheitswesen, Bd. 4, Nr. 4, S. 127-132.

Neumann-Duesberg, R. (1998): Die EuGH-Position ist angreifbar, in: Gesundheit und Gesellschaft, Bd. 1, Nr. 10, S. 22-27.

Nink, K.; Schröder, H. (2005): Ökonomische Aspekte des deutschen Arzneimittelmarktes 2004, in: Schwabe, U.; Paffrath, D. (Hrsg.): Arzneiverordnungs-Report 2005, Berlin, S. 191-243.

Novak, M. (2001): EuGH: Übernahme von Krankenhauskosten in anderem Mitgliedstaat II, 12.07.2001, Rs.C-368/98 (Abdon Vanbraekel/ANMC), in: Europäische Zeitschrift für Wirtschaftsrecht (EuZW), Bd. 12, Nr. 15, S. 471-477.

o.V. (1998a): Nach sechs Jahren Ausgabendeckel für die Arzneimittelversorgung: Das stumpf gewordene Instrument der Budgets wird wieder geschärft, Ärzte Zeitung online, 03.12.1998, www.aerztezeitung.de/docs/1998/12/03/221a0601.asp?cat=, (Abrufdatum: 18.12.2005). 
o.V. (1998b): „Wehret den Anfängen!“ Minister Horst Seehofer über seinen Widerstand gegen den europäischen Kassenpatienten und den Druck auf das deutsche Sozialsystem (4. Mai 1998), in: Der Spiegel, S. 36-38.

O.V. (2004): Internetapotheke: Arzneimittelversand rechtens, in: Deutsches Ärzteblatt, Bd. 101, Nr. 40, S. A 2650.

o.V. (2005a): Dienstleistungsrichtlinie: Knappe Mehrheit für umstrittenes Prinzip, in: Europäischer Informationsbrief, Bd. 11, Nr. 6, S. 3-4.

0.V. (2005b): DocMorris wird sich am Preisdumping nicht beteiligen, in: Frankfurter Allgemeine Zeitung (FAZ), 24.01.2005, S. 19.

o.V. (2005c): Gesundheitspolitik: EU will mehr Einfluss, in: Deutsches Ärzteblatt, Bd. 23, Nr. 102, S. A 1629.

o.V. (2005d): Glossar, in: Gesundheit und Gesellschaft, Bd. 8, Nr. 4, S. 25.

0.V. (2005e): Ob Böhmen oder Balaton: Kuren im Ausland sind keine Seltenheit, in: Rhein Zeitung online vom 22.12.2004, rhein-zeitung.de/on/04/12/22/service/gesund heit/t/rzo 1 14298.html, (Abrufdatum: 22.09.2006)).

0.V. (2006): Dienstleistungsfreiheit: Gleiches Recht auch für Ärzte, in: Deutsches Ärzteblatt, Bd. 103, Nr. 26, S. A 1780.

Oberender, P. (2001): Das deutsche Gesundheitswesen im europäischen Wettbewerb, in: Recht und Politik im Gesundheitswesen, Bd. 7, Nr. 3, S. 79-89.

Organisation for Economic Co-operation and Development (OECD) (2005): OECD Health Data 2005, Paris.

Orosz, H. (2004): Europäische Gesundheitspolitik aus Sicht der Länder, in: Gesellschaft für Versicherungswissenschaft und -gestaltung (GVG) (Hrsg.): Offene Methode der Koordinierung im Gesundheitswesen, Köln, S. 23-30.

Österreichisches Bundesinstitut für Gesundheitswesen (ÖBIG) (Hrsg.) (1998): Arzneimittel: Steuerung der Märkte in neun europäischen Ländern, Wien.

Österreichisches Bundesinstitut für Gesundheitswesen (ÖBIG) (Hrsg.) (2001): Benchmarking. Arzneimittelausgaben und Kostendämpfungsstrategien, Wien.

Palm, W.; Nickless, J.; Lawalle, H.; Coheur, A. (2000): Implications of Recent Jurisprudence on the Co-ordination of Health Care Protection Systems, Brüssel.

Pfaff, M.; Nagel, F. (1995): Vergütungsformen in der vertragsärztlichen Versorgung, in: Soziale Sicherheit, Bd. 44, Nr. 2, S. 41-46. 
Pitschas, R. (1994): Die Weiterentwicklung der sozialen Krankenversicherung in Deutschland im Gegenlicht europäischer Gesundheitspolitik, in: Vierteljahresschrift für Sozialrecht, Nr. 2, S. 85-188.

Reding, K.; Müller, W. (1999): Einführung in die allgemeine Steuerlehre, München.

Reinhardt, U.E.; Sandier, S.; Schneider, M. (1986): Die Wirkungen von Vergütungssystemen auf die Einkommen der Ärzte, die Preise und auf die Struktur ärztlicher Leistungen im internationalen Vergleich, Augsburg.

Ribhegge, H. (2005): Europäische Wirtschafts- und Sozialpolitik, viadrina.euvfrankfurt-o.de/_econpol/lehre/eu_wi_so_po/folien_vorl/Europaeische\%20Sozialpolitik \%20.pdf, (Abrufdatum: 30.11.05).

Riedel, D. (2004): EuGH: Krankenkassen keine Unternehmen i.S. des Wettbewerbsrechts - Arzneimittelfestbeträge zulässig, in: Europäische Zeitschrift für Wirtschaftsrecht, Bd. 15, Nr. 8, S. 241-245.

Rosenbrock, R.; Gerlinger, T. (2004): Gesundheitspolitik, Bern [u.a.].

Rosian, I. (2002): Rezepte zur Kostendämpfung bei Arzneimitteln, in: Gesundheit und Gesellschaft / Wissenschaft, Bd. 2, Nr. 2, S. 22-31.

Rosian, I.; Habl, C.; Vogler, S.; Weigl, M. (2001): Arzneimittelausgaben: Strategien zur Kostendämpfung in der Europäischen Union, Wien.

Rosian, I.; Vogler, S.; Weigl, M. (2000): Generika, Wien.

Rychlik, R.; Bundesverband der Pharmazeutischen Industrie (BPI), (2003): Informationen zu Erstattungsprozeduren in der EU, Berlin.

Sachverständigenrat für die Konzertierte Aktion im Gesundheitswesen (SVRKAiG) (1988): Medizinische und ökonomische Orientierung, Jahresgutachten 1988, Baden-Baden.

Sachverständigenrat für die Konzertierte Aktion im Gesundheitswesen (SVRKAiG) (1992): Ausbau in Deutschland und Aufbruch nach Europa, Jahresgutachten 1992, Baden-Baden.

Sachverständigenrat zur Begutachtung der Entwicklung im Gesundheitswesen (2005): Koordination und Qualität im Gesundheitswesen, Gutachten 2005, Stuttgart.

Sauerland, D. (2002): Gesundheitspolitik in Deutschland, Reformbedarf und Entwicklungsperspektiven, Gütersloh.

Schaub, V.E. (2001): Grenzüberschreitende Gesundheitsversorgung in der Europäischen Union: Die gesetzlichen Gesundheitssysteme im Wettbewerb, Baden-Baden. 
Schirmer, H.D. (2003): Rechtliche Möglichkeiten der grenzüberschreitenden Erbringung von Gesundheitsleistungen, in: Jorens, Y.; Schulte, B. (Hrsg.): Grenzüberschreitende Inanspruchnahme von Gesundheitsleistungen im gemeinsamen Markt, BadenBaden.

Schmucker, R. (2003a): Die Offene Methode der Koordinierung als Instrument europäischer Gesundheitspolitik, in: Die Krankenversicherung, Bd. 55, Nr. 7, S. 206-209.

Schmucker, R. (2003b): Europäische Integration und Gesundheitspolitik, Arbeitspapier Nr. 23/2003, Institut für Medizinische Soziologie, Klinikum der Johann Wolfgang Goethe-Universität, Frankfurt am Main.

Schneider, M. (1999): Die deutschen Arzneimittelpreise im europäischen Vergleich: Gutachten für den Verband Forschender Arzneimittelhersteller (VFA) und die Bundesvereinigung Deutscher Apothekerverbände (ABDA), Augsburg.

Schöffski, O. (1995): Die Regulierung des deutschen Apothekenwesens: Eine ökonomische Analyse, Baden-Baden.

Schulenburg, J.-M. Graf v. d. (1981): Systeme der Honorierung frei praktizierender Ärzte und ihre Allokationswirkungen, Tübingen.

Schulenburg, J.-M. Graf v. d. (1992): Preisbildung im Gesundheitswesen, in: Andersen, H.H.; Henke, K.; Schulenburg, J.-M. Graf v. d. (Hrsg.): Basiswissen Gesundheitsökonomie, Berlin, S. 113-133.

Schulte, B. (1998): Europäische Gesundheitspolitik nach dem Vertrag von Amsterdam, in: Staatswissenschaften und Staatspraxis, Bd. 9, Nr. 3, S. 359-395.

Schulte, B. (1999a): Organisationen, Initiativen und Dienste im sozialen Bereich - ein Motor der Sozialpolitik in Europa, überarbeitete Ausführungen eines Impulsreferates zur Internationalen Konferenz im Rahmen der deutschen EU-Präsidentschaft, veranstaltet vom BMSFSJ, der Europäischen Kommission und der BAG der Freien Wohlfahrtspflege, Mai 1999 in Aachen, forum.sozialarbeit.de/europa/0999de.shtml, (Abrufdatum: 14.02.2005).

Schulte, B. (1999b): Zur Kur nach Abano Therme, zum Zahnarzt nach Antwerpen? Europäische Marktfreiheiten und nationales Krankenversicherungsrecht, in: Zeitschrift für Sozialhilfe und Sozialgesetzbuch, Bd. 38, Nr. 5 und 6, S. 269-278 und 347-362.

Schulte, B. (2000): Europäisches Wirtschaftsrecht und die Grundfreiheiten in der EU als Rahmenbedingungen für die nationale Sozialgesetzgebung - Erfahrungen in der Vergangenheit, Perspektiven für die Zukunft -, in: Ebsen, I. (Hrsg.): Europarechtliche Gestaltungsvorgaben für das deutsche Sozialrecht, Baden-Baden. 
Schulte, B. (2005): Europäische Vorgaben für die nationalen Gesundheitssysteme: Ziele und Instrumente, in: Gesundheit und Gesellschaft / Wissenschaft, Bd. 5, Nr. 4, S. 15-25.

Schwanenflügel, M.v. (1996): Die Entwicklung der Kompetenzen der EU im Gesundheitswesen, Berlin.

Schwarze, J.; Andersen, H.H. (2001): Kassenwechsel in der Gesetzlichen Krankenversicherung: Welche Rolle spielt der Beitragssatz? in: Schmollers Jahrbuch: Zeitschrift für Wirtschafts- und Sozialwissenschaften, Bd. 121, Nr. 4, S. 584-602.

Seliger, B.; Herrmann-Pillath, C. (2000): Wettbewerb auf Gütermärkten, politischer Wettbewerb und Systemwettbewerb, in: Wirtschaftsstudium, Bd. 29, Nr. 8/9, S. 11481155.

Service Public Portail de l'Administration Française (2007): Portail de l'Administration Française, www.service-public.fr/, (Abrufdatum: 10.03.2007).

Service Public. Portail de l'Administration Française (2006): Vos droits et démarches: Santé, Cure thermal, vosdroits.service-public.fr/particuliers /F751.xhtml? $\& \mathrm{n}=\mathrm{Sant} \% \mathrm{C} 3 \% \mathrm{~A} 9 \& \mathrm{l}=\mathrm{N} 17 \& \mathrm{n}=\mathrm{S} \% \mathrm{C} 3 \% \mathrm{~A} 9 \mathrm{curit} \% \mathrm{C} 3 \% \mathrm{~A} 9 \% 20$ sociale $\& \mathrm{l}=\mathrm{N} 416 \& \mathrm{n}=\mathrm{S}$ \%C3\%A9curit\%C3\%A9\%20sociale:\%20r\%C3\%A9gime\%20g\%C3\%A9n\%C3\%A9ra $1 \& \mathrm{l}=\mathrm{N} 424 \& \mathrm{n}=\mathrm{Cure} \% 20$ thermale $\& \mathrm{l}=\mathrm{N} 430$, (Abrufdatum: 26.7 .06 ).

Sinn, H. (1994): How much Europe?: Subsidiarity, centralization and fiscal competition, in: Scottish Journal of Political Economy, Bd. 41, Nr. 1, S. 85-107.

Sozialministerium Baden-Württemberg (2003): Baden-Württemberg im Europa der Regionen: Dimensionen und Perspektiven bei grenzübergreifenden Gesundheitsleistungen, Stuttgart.

Spalcke, J. (2004): Arzneimittelzulassungsverfahren in der Europäischen Union und den Vereinigten Staaten von Amerika, Frankfurt am Main [u.a.].

Spiegel Online (2006): NC-Flucht nach Österreich - Piefkefilter für Mediziner, www.spiegel.de/unispiegel/studium/0,1518,402458,00.html, (Abrufdatum: 26.09. 2006).

Spielberg, P. (2004): Wenn Ausnahmen zur Regel werden, in: Deutsches Ärzteblatt, Bd. 101, Nr. 47, S. A 3148.

Spielberg, P. (2006): EU-Dienstleistungssrichtlinie: Gesundheit ausgenommen, in: Deutsches Ärzteblatt, Bd. 108, Nr. 8, S. A 454.

Spitzenverbände der Krankenkassen (2005): Gemeinsame Erklärung der Spitzenverbände der Krankenkassen zu leistungsrechtlichen Umsetzungsfragen, hier: Vorsorge- und Rehabilitationsleistungen im Ausland, o.O. 
Staffeldt, T. (2004): Morbiditätsbezogene Regelleistungsvolumina in der ärztlich Vergütung: eine Machbarkeitsanalyse, in: Gesundheits- und Sozialpolitik, Bd. 58, Nr. $11 / 12$, S. 51-59.

Statistisches Bundesamt (2006): Gesundheitswesen, www.destatis.de/themen/d/thm _ gesundheit.php, (Abrufdatum: 29.09.2006).

Statistisches Bundesamt (Hrsg.) (2004): Datenreport 2004: Fakten und Daten über die Bundesrepublik Deutschland, Wiesbaden.

Statistisches Bundesamt (Hrsg.) (2006): Datenreport 2006: Fakten und Daten über die Bundesrepublik Deutschland, Wiesbaden.

Streit, M.E.; Mussler, W. (1995): Wettbewerb der Systeme und das Binnenmarktprogramm der Europäischen Union, in: Gerken, L. (Hrsg.): Europa zwischen Ordnungswettbewerb und Harmonisierung: Europäische Ordnungspolitik im Zeichen der Subsidiarität, Berlin, S. 75-107.

Sundmacher, T. (2005a): Die Offene Methode der Koordinierung im Gesundheitswesen: Folgenlose Verschwendung oder Gewinne jenseits ausgetretener Pfade? in: Sozialer Fortschritt, Bd. 53, Nr. 12, S. 304-310.

Sundmacher, T. (2005b): Europäischer „Sozialschutz“ und die „neue“ offene Methode der Koordinierung, Diskussionspapier, Fachbereich Betriebswirtschaftslehre, Allgemeine Wirtschaftspolitik, Universität Duisburg-Essen.

Techniker Krankenkasse (TK) (2003): Medizin und Europa, Ergebnisse der TKMitglieder-Befragung 2003, Hamburg.

Tiemann, S. (2004): Offene Methode der Koordinierung und Streamlining aus Sicht der GVG, in: Gesellschaft für Versicherungswissenschaft und -gestaltung (GVG) (Hrsg.): Offene Methode der Koordinierung im Gesundheitswesen, Köln, S. 95-104.

Verband der Angestellten-Krankenkassen (VdAK) (2006): Basisdaten 2005, Siegburg.

Verband der Forschenden Arzneimittelhersteller (VFA) (2004): Parallel- und Reimporte im Arzneimittelmarkt: Gesundheitspolitik auf dem Irrweg, www.vfa.de/download/de/politik/artikelpo/reimporte.html/reimporte.pdf, (Abrufdatum: 14.07.2005).

Verband Forschender Arzneimittelhersteller (VFA) (Hrsg) (2006): Statistics. Die Arzneimittelindustrie in Deutschland 2006, Berlin. 
Verordnung (EWG) 1408/71 (1971): des Rates vom 14. Juni 1971 über die Anwendung des Systems der sozialen Sicherheit auf Arbeitnehmer und Selbständige sowie deren Familienangehörige, die innerhalb der Gemeinschaft zu- und abwandern, in: ABl. EG Nr. L 149 vom 05.07.1971, S. 2-50.

Verordnung (EWG) 574/72 (1972): über die Durchführung der Verordnung (EWG) Nr. 1408/71, in: ABl. EG Nr. L 74 vom 27.03.1972, S. 1-83.

Wasem, J.; GreB, S.; Niebuhr, D. (2005): Regulierung des Marktes für verschreibungspflichtige Arzneimittel im internationalen Vergleich, Gutachten im Auftrag des Bundesverbandes der Arzneimittelhersteller, Essen.

Weber, I.; Brenner, G.; Weisner, E. (2001): Gewogen - und zu leicht befunden, in: Deutsches Ärzteblatt, Bd. 98, Nr. 45, S. A 2932-A 2936.

Weingärtner, T. (2000): Im Visier der Kommissare, in: Gesundheit und Gesellschaft, Nr. 11, S. 32-35.

Weis, H. (1983): Inländerdiskriminierung zwischen Gemeinschaftsrecht und nationalem Verfassungsrecht, in: Neue Juristische Wochenschrift, Bd. 36, Nr. 48, S. 27212726.

Weisbrod, B.A. (1964): Collective-Consumption Services of Individual-Consumption Goods, in: Quarterly Journal of Economics, Bd. 78, Nr. 3, S. 471-477.

Westdeutscher Rundfunk (WDR) (2004): AOK Rheinland schließt Vertrag mit der Internetapotheke Doc Morris, www.wdr.de/themen/gesundheit/pharmazie/doc_morris/ vertrag_aok_rheinland.jhtml, (Abrufdatum: 4.11.2005).

Wikepedia (2006): Europäische Patentorganisation, de.wikipedia.org/wiki/Europ \%C3\%A4ische_Patentorganisation, (Abrufdatum: 29.09.2006).

Wille, E. (1998): Mögliche Auswirkungen der Europäischen Währungsunion auf die Sozial-, insbesondere die gesetzliche Krankenversicherung, in: Staatswissenschaften und Staatspraxis, Bd. 9, Nr. 3, S. 343-358.

Wille, E. (2002): Editorial, in: Wille, E. (Hrsg.): Anreizkompatible Vergütungssysteme im Gesundheitswesen, Baden-Baden, S. 5-8.

Wille, E. (2005): Auswirkungen der europäischen Integrationspolitik auf das deutsche Gesundheitswesen, in: Allgemeine Ortskrankenkasse (AOK) (Hrsg.): AOK im Dialog: Europa für die Versicherten gestalten, Bonn, S. 29-47.

Wille, E.; Knerr, C. (2000): Gutachten: Ökonomische Bewertung der Handlungsoptionen, in: Gesellschaft für Versicherungswissenschaft und -gestaltung (GVG) (Hrsg.): Gutachten: Handlungsoptionen der Techniker Krankenkasse bei Leistungen im Ausland - Abschlussbericht-, Köln, S. 247-290. 
Wismar, M. (1998): Europa regiert schon lange heimlich mit, in: Gesundheit und Gesellschaft, Bd. 1, Nr. 10, S. S. 28-35.

Wohlgemuth, M. (1998): Institutioneller Wettbewerb als Entdeckungsverfahren: Zur Rolle von Abwanderung und Widerspruch im europäischen Binnenmarkt, in: König, T.; Rieger, E.; Schmitt, H. (Hrsg.): Europa der Bürger? Voraussetzungen, Alternativen, Konsequenzen, Frankfurt [u.a.].

World Health Organization (WHO) (2000): The World Health Report 2000. Health Systems: Improving Performance, Genf.

World Health Organization. (WHO) (2005): Core Health Indicators, www3.who.int/whosis/core/core_select.cfm?path=whosis,core\&language=english, (Abrufdatum: 24.11.2005). 


\section{STAATLICHE ALLOKATIONSPOLITIK IM MARKTWIRTSCHAFTLICHEN SYSTEM}

Band 1 Horst Siebert (Hrsg.): Umweltallokation im Raum. 1982.

Band 2 Horst Siebert (Hrsg.): Global Environmental Resources. The Ozone Problem. 1982.

Band 3 Hans-Joachim Schulz: Steuerwirkungen in einem dynamischen Unternehmensmodell. Ein Beitrag zur Dynamisierung der Steuerüberwälzungsanalyse. 1981.

Band 4 Eberhard Wille (Hrsg.): Beitrăge zur gesamtwirtschaftlichen Allokation. Allokationsprobleme im intermediåren Bereich zwischen ठffentlichem und privatem Wirtschaftssektor. 1983.

Band 5 Heinz König (Hrsg.): Ausbildung und Arbeitsmarkt. 1983.

Band 6 Horst Siebert (Hrsg.): Reaktionen auf Energiepreissteigerungen. 1982.

Band 7 Eberhard Wille (Hrsg.): Konzeptionelle Probleme offentlicher Planung. 1983.

Band 8 Ingeborg Kiesewetter-Wrana: Exporterlösinstabilităt. Kritische Analyse eines entwicklungspolitischen Problems. 1982.

Band 9 Ferdinand Dudenhöfer: Mehrheitswahl-Entscheldungen über Umweltnutzungen. Eine Untersuchung von Gleichgewichtszuständen in einem mikroökonomischen Markt- und Abstimmungsmodell. 1983.

Band 10 Horst Siebert (Hrsg.): Intertemporale Allokation. 1984.

Band 11 Helmut Meder: Die intertemporale Allokation erschöpfbarer Naturressourcen bel fehlenden Zukunftsmärkten und institutionalisierten Marktsubstituten. 1984.

Band 12 Ulrich Ring: Öffentliche Planungsziele und staatliche Budgets. Zur Erfüllung offentlicher Aufgaben durch nicht-staatliche Entscheidungseinheiten. 1985.

Band 13 Ehrentraud Graw: Informationseffizienz von Terminkontraktmärkten für Währungen. Eine empirische Untersuchung. 1984.

Band 14 Rüdiger Pethig (Ed.): Public Goods and Public Allocation Policy. 1985.

Band 15 Eberhard Wille (Hrsg.): Öffentliche Planung auf Landesebene. Eine Analyse von Planungskonzepten in Deutschland, Ósterreich und der Schweiz. 1986.

Band 16 Helga Gebauer: Regionale Umweltnutzungen in der Zeit. Eine intertemporale Zwei-Regionen-Analyse. 1985.

Band 17 Christine Pfitzer: Integrierte Entwicklungsplanung als Allokationsinstrument auf Landesebene. Eine Analyse der öffentlichen Planung der Länder Hessen, Bayern und Niedersachsen. 1985.

Band 18 Heinz Kőnig (Hrsg.): Kontrolltheoretische Ansătze in makroőkonometrischen Modellen. 1985.

Band 19 Theo Kempf: Theorie und Empirie betrieblicher Ausbildungsplatzangebote. 1985.

Band 20 Eberhard Wille (Hrsg.): Konkrete Probleme offentlicher Planung. Grundlegende Aspekte der Zlelbildung, Effizienz und Kontrolle. 1986.

Band 21 Eberhard Wille (Hrsg.): Informations- und Planungsprobleme in öfentlichen Aufgabenberejchen. Aspekte der Zlelbildung und Outputmessung unter besonderer Berücksichtigung des Gesundheitswesens. 1986.

Band 22 Bernd Gutting: Der Einfluß der Besteuerung auf die Entwicklung der Wohnungs- und Baulandmärkte. Eine intertemporale Analyse der bundesdeutschen Steuergesetze. 1986.

Band 23 Heiner Kuhl: Umweltressourcen als Gegenstand intemationaler Verhandlungen. Eine theoretische Transaktionskostenanalyse. 1987.

Band 24 Hubert Hornbach: Besteuerung, Inflation und Kapitalallokation. Intersektorale und internationale Aspekte. 1987. 
Band 25 Peter Müller: Intertemporale Wirkungen der Staatsverschuldung. 1987.

Band 26 Stefan Kronenberger: Die Investitionen im Rahmen der Staatsausgaben. 1988.

Band 27 Armin-Detlef Rieß: Optimale Auslandsverschuldung bei potentiellen Schuldendienstproblemen. 1988.

Band 28 Volker Uirich: Preis- und Mengeneffekte im Gesundheltswesen. Eine Ausgabenanalyse von GKV-Behandlungsarten. 1988.

Band 29 Hans-Michael Geiger: Informational Efficiency in Speculative Markets. A Theoretical Investigation. Edited by Ehrentraud Graw. 1989.

Band 30 Karl Sputek: Zlelgerichtete Ressourcenallokation. Ein Modellentwurf zur Effektlvitătsanalyse praktischer Budgetplanung am Beispiel von Berlin (West). 1989.

\section{ALLOKATION IM MARKTWIRTSCHAFTLICHEN SYSTEM}

Band 31 Wolfgang Krader: Neuere Entwicklungen linearer latenter Kovarianzstrukturmodelle mit quantitativen und qualitativen Indikatorvariablen. Theorie und Anwendung auf ein mikroempirisches Modell des Preis-, Produktions- und Lageranpassungsverhaltens von deutschen und französischen Unternehmen des verarbeitenden Gewerbes. 1991.

Band 32 Manfred Erbsland: Die öffentlichen Personalausgaben. Eine empirische Analyse für die Bundesrepublik Deutschland. 1991.

Band 33 Walter Ried: Information und Nutzen der medizinischen Diagnostik. 1992.

Band 34 Anselm U. Römer: Was ist den Bürgern die Verminderung eines Risikos wert? Eine Anwendung des kontingenten Bewertungsansatzes auf das Giftmüllisiko. 1993.

Band 35 Eberhard Wille, Angelika Mehnert, Jan Philipp Rohweder: Zum gesellschaftlichen Nutzen pharmazeutischer Innovationen. 1994.

Band 36 Peter Schmidt: Die Wahl des Rentenalters. Theoretische und empirische Analyse des Rentenzugangsverhaltens in West- und Ostdeutschland. 1995.

Band 37 Michael Ohmer: Die Grundlagen der Einkommensteuer. Gerechtigkeit und Effizienz. 1997.

Band 38 Evamaria Wagner: Risikomanagement rohstoffexportierender Entwicklungslănder. 1997.

Band 39 Matthias Meler: Das Sparverhalten der privaten Haushalte und der demographische Wandel: Makroökonomische Auswirkungen. Elne Simulation verschiedener Reformen der Rentenversicherung. 1997.

Band 40 Manfred Albring / Eberhard Wille (Hrsg.): Innovationen in der Arzneimitteltherapie. Definition, medizinische Umsetzung und Finanzierung. Bad Orber Gespräche über kontroverse Themen Im Gesundheitswesen 25.-27.10.1996. 1997.

Band 41 Eberhard Wille / Manfred Albring (Hrsg.): Reformoptionen im Gesundheitswesen. Bad Orber Gespräche über kontroverse Themen im Gesundheitswesen 7.-8.11.1997. 1998.

Band 42 Manfred Albring / Eberhard Wille (Hrsg.): Szenarien im Gesundheitswesen. Bad Orber Gespräche über kontroverse Themen im Gesundheitswesen 5.-7.11.1998. 1999.

Band 43 Eberhard Wille / Manfred Albring (Hrsg.): Rationalisierungsreserven im deutschen Gesundheltswesen. 2000.

Band 44 Manfred Albring / Eberhard Wille (Hrsg.): Qualitătsorientierte Vergütungssysteme in der ambulanten und stationăren Behandlung. 2001.

Band 45 Martin Pfaft / Dietmar Wassener / Astrid Sterzel / Thomas Neldner: Analyse potentieller Auswirkungen einer Ausweitung des Pharmaversandes in Deutschland. 2002.

Band 46 Eberhard Wille / Manfred Albring (Hrsg.): Konfliktfeld Arzneimittelversorgung. 2002. 
Band 47 Udo Schneider: Theorie und Empirie der Arzt-Patient-Beziehung. Zur Anwendung der Principal-Agent-Theorie auf die Gesundheitsnachírage. 2002.

Band 48 Manfred Albring / Eberhard Wille: Die GKV zwischen Ausgabendynamik, Einnahmenschwăche und Koordinierungsproblemen. 2003.

Band 49 Uwe Jirjahn: X-Ineffizienz, Managementanreize und Produktmarktwettbewerb. 2004.

Band 50 Stefan Resch: Risikoselektion im Mitgliederwettbewerb der Gesetzlichen Krankenversicherung. 2004.

Band 51 Paul Marschall: Lebensstilwandel in Ostdeutschland. Gesundheitsökonomische Implikationen. 2004.

Band 52 Eberhard Wille / Manfred Albring (Hrsg.): Paradigmenwechsel im Gesundheitswesen durch neue Versorgungsstrukturen? 8. Bad Orber Gesprăche. 6. - 8. November 2003. 2004.

Band 53 Eberhard Wille / Manfred Albring (Hrsg.): Versorgungsstrukturen und Finanzierungsoptionen auf dem Prüfstand. 9. Bad Orber Gesprăche. 11.-13. November 2004. 2005.

Band 54 Brit S. Schneider: Gesundheit und Bildung. Theorie und Empirie der Humankapitalinvestitionen. 2007.

Band 55 Klaus Knabner / Eberhard Wille (Hrsg.): Qualităt und Nutzen medizinischer Leistungen. 10. Bad Orber Gesprăche, 10.-12. November 2005. 2007.

Band 56 Holger Cischinsky: Lebensenwartung, Morbidität und Gesundheitsausgaben. 2007.

Band 57 Eberhard Wille / Klaus Knabner (Hrsg.): Wettbewerb im Gesundheitswesen: Chancen und Grenzen. 11. Bad Orber Gesprăche. 16. -18. November 2006. 2008.

Band 58 Christian Igel: Zur Finanzierung von Kranken- und Pflegeversicherung. Entwicklung, Probleme und Reformmodelle. 2008.

Band 59 Christiane Cischinsky: Auswirkungen der Europäischen Integration auf das deutsche Gesundheitswesen. 2008.

uww.peterlang.de 


\section{Paradigmenwechsel im Gesundheitswesen durch neue Versorgungsstrukturen?}

\section{Bad Orber Gespräche \\ 6. - 8. November 2003}

Frankfurt am Main, Berlin, Bern, Bruxelles, New York, Oxford, Wien, 2004. 306 S., zahlr. Abb. und Tab.

Allokation im marktwirtschaftlichen System.

Verantwortlicher Herausgeber: Eberhard Wille. Bd. 52

ISBN 3-631-53394-2 · br. $€ 40.70 *$

Der Sammelband enthält die erweiterten Referate eines interdisziplinären Workshops zur Frage, inwiefern sich durch neue Versorgungsstrukturen ein Paradigmenwechsel im Gesundheitswesen vollzieht. Diskutiert werden die beiden Themenkreise Vertragswettbewerb sowie Effizienz und Effektivität der Arzneimitteltherapie.

Aus dem Inhalt. P. Zweifel: Was können wir vom Schweizer Gesundheitswesen lernen? $\cdot H$. Sodan / I. Ebsen: Selektives Kontrahieren unter wettbewerbspolitischen Gesichtspunkten · H. J. Ahrens: Umsetzung der integrierten Versorgung bis zu Einzelverträgen $\cdot K$. $-H$. Schönbach: Integrierte Versorgung - J. Bausch / K. -D. Kossow. Vertragswettbewerb und ärztliche Vergütung $\cdot H$. Lohmann: Auswirkungen von Vertragswettbewerb auf Krankenhäuser: Vom Budget- zum Vertragssystem - W. Gerdelmann I W. Schmeinck: Paradigmenwechsel im Gesundheitswesen durch neue Versorgungsstrukturen? · F. Münnich: Auswirkungen der Preisbildungs- und Erstattungs-Neuregelung im Arzneimittelbereich - C. Straub: Interdependenzen zwischen Disease-Management-Programmen und Vertragswettbewerb . R. Hoberg: Möglichkeiten des Vertragswettbewerbs im Rahmen von DiseaseManagement-Programmen - U. Schwabe / N. Schmacke: Bewertung des Nutzens aus ärztlicher Sicht · u.v.m.

Frankfurt am Main - Berlin - Bern - Bruxelles - New York - Oxford - Wien

Auslieferung: Verlag Peter Lang AG

Moosstr. 1, CH-2542 Pieterlen

Telefax 0041 (0) 32/3761727

*inklusive der in Deutschland gültigen Mehrwertsteuer

Preisänderungen vorbehalten

Homepage http://www.peterlang.de 
